\title{
Estimating Molecular Weights of Organometallics in Solution with Diffusion NMR Techniques
}

\author{
Dissertation \\ zur \\ Erlangung des mathematisch-naturwissenschaftlichen Doktorgrades \\ „Doctor rerum naturalium“ \\ der Georg-August-Universität Göttingen
}

im Promotionsprogramm Chemie

der Georg-August University School of Science (GAUSS)

vorgelegt von

Sebastian Bachmann

aus Goslar

Göttingen, 2017 


\section{Betreuungsausschuss:}

Prof. Dr. Dietmar Stalke, Institut für Anorganische Chemie

Dr. Michael John, Institut für Organische und Biomolekulare Chemie

\section{Mitglieder der Prüfungskommission:}

Referent: $\quad$ Prof. Dr. Dietmar Stalke, Institut für Anorganische Chemie

Korreferent: $\quad$ Prof. Dr. Konrad Koszinowski, Institut für Organische und Biomolekulare Chemie

weitere Mitglieder der Prüfungskommission:

Dr. Michael John, Institut für Organische und Biomolekulare Chemie

Prof. Dr. Ricardo Mata, Institut für Physikalische Chemie

Prof. Dr. Inke Siewert, Institut für Anorganische Chemie

Jun.-Prof. Dr. Selvan Demir, Institut für Anorganische Chemie

Tag der mündlichen Prüfung: 01.03.2017 
für Anna Kristina

There is no real ending. It's just the place where you stop the story. Frank Herbert 


\section{TABLE OF CONTENTS}

\section{ABbREVIATIONS}

1 INTRODUCTION

1.1 Organometallic Chemistry 1

1.1.1 Lithiumorganics 1

1.1.1.1 Relationship of Structure and Reactivity 2

1.1.1.2 Solvent-Separated Ion Pairs (SSIPs) 3

1.1.2 Alkali Metal Cyclopentadienides (CpMs) 4

1.1.2.1 A Short Historical Background 4

1.1.2.2 Practical Applications 4

1.1.2.3 Properties and Bonding 5

1.1.2.4 Aggregation of CpMs in the Solid State and in Solution 6

1.2 Nuclear Magnetic Resonance (NMR) Spectroscopy 8

1.2.1.1 Heteronuclear Overhauser Enhancement Spectroscopy (HOESY) 9

$\begin{array}{lll}\text { 1.2.2 Overview of Diffusion NMR } & 10\end{array}$

$\begin{array}{lll}\text { 1.2.2.1 Calculating Diffusion Coefficients } & 11\end{array}$

1.2.2.2 Advances in Diffusion NMR 12

$\begin{array}{lll}\text { 1.2.2.3 Diffusion-Ordered Spectroscopy (DOSY) } & 12\end{array}$

1.2.3 Molecular Sizes and Weights from Diffusion Coefficients 13

1.2.3.1 MW Estimation Using Power Law Approaches $\quad 15$

$\begin{array}{lll}1.3 & \text { Scope of this Thesis } & 19\end{array}$

2 RESULTS AND DISCUSSION

2.1 Improving the External Calibration Curve (ECC)-DOSY-MW Estimation Methodology 21

2.1.1 New External Calibration Curves for Various Solvents $\quad 21$

2.1.1.1 Merged External Calibration Curves 23

2.1.1.2 Shape-Optimized External Calibration Curves 28

2.1.2 Molecule Categorization 33

2.1.3 Theoretical Error Analysis 36

2.1.4 Heavy Atoms in the ECC-DOSY-MW Estimation 38

2.1.5 MW Estimation Software $\quad 41$

2.2 Structure Elucidation of Alkali Metal Cyclopentadienide Derivatives via Diffusion NMR 44

2.2.1 Solution State Structures of Alkali Metal Cyclopentadienides (CpMs) in Different Solvents 44

2.2.1.1 Solution State Structures of CpMs in THF- $d_{8}$

2.2.1.2 Solution State Structures of CpMs in ammonia and DMSO- $d_{6} \quad 49$

2.2.2 Solution State Structures of Methylated Alkali Metal Cyclopentadienides in THF- $d_{8} \quad 53$

2.2.2.1 Solution State Structure of MeCpLi in THF- $d_{8}$

2.2.2.2 Solution State Structures of Cp $\mathrm{p}^{\star} \mathrm{Ms}$ in THF- $d_{8}$

2.3 Combined Studies Involving Diffusion NMR to Solve Structural Ambiguities 56

2.3.1 Solution State Structures of Alkyl Grignard Reagents in THF- $d_{8} \quad 56$

2.3.1.1 External Calibration Curves for Rod-Like (RL) Molecules 59

2.3.1.2 MW Estimation of Alkyl Grignard Reagents from Diffusion Data 61 
2.3.1.3 Solution State Structures of the "Turbo"-Grignard Analogues 63

2.3.2 Anion and Solvent Coordination Elucidated via DOSY 65

2.3.2.1 Coordination of Different Anions to an N-Heterocyclic Carbene in $\mathrm{CD}_{2} \mathrm{Cl}_{2} \quad 66$

2.3.2.2 Solvent-Anion Exchange of an Acridine-Based System in DMF- $d_{7} \quad 68$

2.3.2.3 Solvation of a Heterocyclic Substituted Methanide in $\mathrm{C}_{6} \mathrm{D}_{6} \quad 72$

3 SUMMARY \& OUTLOOK $\quad 76$

4 EXPERIMENTAL PART $r$

$\begin{array}{llr}4.1 & \text { Techniques and Experiments } & 81\end{array}$

4.1.1 Handling of Air- and Moisture-Sensitive Compounds $\quad 81$

$\begin{array}{ll}\text { 4.1.2 NMR Techniques and Experiments } & 81\end{array}$

4.1.3 DOSY Sample Preparation and Evaluation 82

4.1.4 Computation of Principal Moments of Inertia $\quad 83$

$\begin{array}{ll}\text { 4.1.5 Programming in Java } & 83\end{array}$

4.2 Syntheses and Characterizations $\quad 84$

4.2.1 Syntheses and Characterizations of CpMs $(\mathrm{M}=\mathrm{Li}, \mathrm{Na}, \mathrm{K}, \mathrm{Rb}, \mathrm{Cs})$

4.2.1.1 Synthesis and Characterization of CpLi 84

4.2.1.2 Synthesis and Characterization of $\mathrm{CpNa} 86$

4.2.1.3 Synthesis and Characterization of $\mathrm{CpK} \quad 87$

4.2.1.4 Synthesis and Characterization of $\mathrm{CpRb} \quad 88$

4.2.1.5 Synthesis and Characterization of CpCs 89

4.2.2 Synthesis and Characterization of MeCpLi 90

4.2.2.1 Synthesis and Characterization of MeCpLi 90

4.2.3 Syntheses and Characterizations of $\mathrm{Cp}^{\star} \mathrm{M}(\mathrm{M}=\mathrm{Li}, \mathrm{K}, \mathrm{Cs}) \quad 91$

4.2.3.1 Synthesis of $\mathrm{Cp}^{\star} \mathrm{Li} \quad 91$

4.2.3.2 Synthesis and Characterization of $\mathrm{Cp}^{\star} \mathrm{K} \quad 91$

4.2.3.3 Characterization of $\mathrm{Cp}^{\star} \mathrm{Cs} \quad 92$

4.2.4 Characterizations of $\mathrm{RMgCl}(\mathrm{R}=\mathrm{Et}, i \mathrm{Pr}, n \mathrm{Bu}, n \mathrm{Hex}, n \mathrm{Oct}, n \mathrm{Dec})$

4.2.4.1 Characterization of EtMgCl 93

4.2.4.2 Characterization of $i \mathrm{PrMgCl} 93$

4.2.4.3 Characterization of $n \mathrm{BuMgCl}$

4.2.4.4 Characterization of $n \mathrm{HexMgCl}$

4.2.4.5 Characterization of $n \mathrm{OctMgCl}$

4.2.4.6 Characterization of $n \mathrm{DecMgCl}$

$\begin{array}{lll}\text { 4.2.5 Characterization of } n \mathrm{BuMgBr} & 95\end{array}$

4.2.5.1 Characterization of $n \mathrm{BuMgBr} \quad 95$

4.2.6 Characterizations of $[n \mathrm{BuMgX} \cdot \mathrm{LiX}](\mathrm{X}=\mathrm{Cl}, \mathrm{Br}) \quad 96$

4.2.6.1 Characterization of $[n \mathrm{BuMgCl} \cdot \mathrm{LiCl}] \quad 96$

4.2.6.2 Characterization of $[n \mathrm{BuMgBr} \cdot \mathrm{LiBr}] \quad 96$

4.2.7 Characterizations of $\left[\mathrm{MX}_{2}\left\{\left(\mathrm{Me}_{2} \mathrm{NCH}_{2}\right)_{2} \mathrm{Acr}\right\}\right]\left(\mathrm{MX}_{2}=\mathrm{ZnBr}(\mathrm{DMF}), \mathrm{CdBr}_{2}\right)$

4.2.7.1 Characterization of $\left[\mathrm{ZnBr}(\mathrm{DMF})\left\{\left(\mathrm{Me}_{2} \mathrm{NCH}_{2}\right)_{2} \mathrm{Acr}\right\}\right] \quad 97$

4.2.7.2 Characterization of $\left[\mathrm{CdBr}_{2}\left\{\left(\mathrm{Me}_{2} \mathrm{NCH}_{2}\right)_{2} \mathrm{Acr}\right\}\right]$ 
6 REFERENCES $\quad 144$

DANKSAGUNG 154

CURRICULUM VITAE 


\section{ABBREVIATIONS}

\begin{tabular}{|c|c|}
\hline $1-\mathrm{PhN}$ & 1-phenylnaphthalene \\
\hline 2-PhP & 2-phenylpyridine \\
\hline 9-MA & 9-methylanthracene \\
\hline Ac & acetyl \\
\hline Acr & acridine \\
\hline Adam & adamantane \\
\hline av. & average \\
\hline Batyl & batylalcohol \\
\hline BEN & benzene \\
\hline BINAP & 2,2'-bis(diphenylphosphino)-1,1'-binaphthyl \\
\hline $\mathrm{Bn}$ & benzyl \\
\hline BPP-LDE & bipolar pulse pairs with longitudinal-eddy-current-delay \\
\hline CIP & contact ion pair \\
\hline $\mathrm{COE}$ & cyclooctene \\
\hline cor. & correlated \\
\hline $\cos Y$ & correlation spectroscopy \\
\hline $\mathrm{Cp} / \mathrm{Cp}^{-}$ & cyclopentadiene/cyclopentadienide \\
\hline $\mathrm{Cp}^{*} / \mathrm{Cp}^{*-}$ & pentamethylcyclopentadiene/pentamethylcyclopentadienide \\
\hline CpM & alkali metal cyclopentadienide \\
\hline CS & compact spheres/compact spherical \\
\hline Cycl & cyclohexane- $d_{12}$ \\
\hline dev. & deviation(s) \\
\hline DIPA & di-iso-propylamine \\
\hline DHBP & 3,4-dihydrobenzophenanthrene \\
\hline DMF & dimethylformamide \\
\hline DMSO & dimethylsulfoxide \\
\hline DOSY & diffusion-ordered spectroscopy \\
\hline DPA & diphenylacetylene \\
\hline DPS & diphenylsulfoxide \\
\hline ds & dummy scans \\
\hline DSE & dissipated spheres and ellipsoids \\
\hline D-STE & double-stimulated-echo \\
\hline ECC & external calibration curve \\
\hline ED & expanded discs \\
\hline ESI & electrospray-ionization \\
\hline Et & ethyl \\
\hline $\mathrm{Et}_{2} \mathrm{O}$ & diethyl ether \\
\hline GUI & graphical user interface \\
\hline HOESY & heteronuclear Overhauser enhancement spectroscopy \\
\hline
\end{tabular}




\begin{tabular}{|c|c|}
\hline HMBC & heteronuclear multiple bond correlation \\
\hline HMDS & hexamethyldisilazane \\
\hline HMPA & hexamethylphosphoramide \\
\hline HSQC & heteronuclear single quantum correlation \\
\hline ICC & internal calibration curve \\
\hline$i \operatorname{Pr}$ & iso-propyl \\
\hline $\mathrm{IPr}$ & 1,3-bis(2,6-diisopropylphenyl)imidazol-2-yliden \\
\hline$i \mathrm{Pr}_{2} \mathrm{O}$ & di-iso-propylether \\
\hline IR & infrared \\
\hline JVM & Java virtual machine \\
\hline LIFDI & liquid injection field desorption ionization \\
\hline MAO & methylaluminoxane \\
\hline MAS & magic angle spinning \\
\hline $\max$. & maximum \\
\hline $\mathrm{Me}$ & methyl \\
\hline $\mathrm{MeCp} / \mathrm{MeCp}^{-}$ & methylcyclopentadiene/methylcyclopentadienide \\
\hline $\mathrm{Mp}$ & melting point \\
\hline MS & mass spectrometry \\
\hline MTBE & methyl-tert-butylether \\
\hline$M W$ & molecular weight \\
\hline$n \mathrm{Bu}$ & $n$-butyl \\
\hline$n \mathrm{Dec}$ & $n$-decyl \\
\hline NHC & $\mathrm{N}$-heterocyclic carbene \\
\hline$n \mathrm{Hex}$ & $n$-hexyl \\
\hline$n$ Oct & $n$-octyl \\
\hline$n$ Pen & $n$-pentyl \\
\hline$n \operatorname{Pr}$ & $n$-propyl \\
\hline NMR & nuclear magnetic resonance \\
\hline NOE & nuclear Overhauser effect \\
\hline NOESY & nuclear Overhauser enhancement spectroscopy \\
\hline OTf & triflate \\
\hline PFG-SE & pulsed-field gradient spin-echo \\
\hline PFG-STE & pulsed-field gradient stimulated-echo \\
\hline $\mathrm{Ph}$ & phenyl \\
\hline PMDETA & $N, N, N^{\prime}, N^{\prime \prime}, N^{\prime \prime}$-pentamethyldiethylenetriamine \\
\hline $\mathrm{R}$ & residue \\
\hline RINMR & rapid injection nuclear magnetic resonance \\
\hline RL & rod-like \\
\hline SSIP & solvent-separated ion pair \\
\hline TBACl & tetrabutylammoniumchloride \\
\hline TBDBS & tert-butyldiphenylsilyl \\
\hline$s \mathrm{Bu}$ & sec-butyl \\
\hline
\end{tabular}


Abbreviations

$t \mathrm{Bu}$

TDE

TMB

TMEDA

TMP

THF

TOF

Tol

$\mathrm{TPhN}$

UCC

UV

VIS tert-butyl

1-tetradecene

2,2,3,3-tetramethylbutane

$N, N, N$ ',N'-tetramethylethylene-1,2-diamine

2,2,6,6-tetramethylpiperidide

tetrahydrofurane

time of flight

toluene

1,2,3,4-tetraphenylnaphthalene

universal calibration curve

ultraviolet

visible 
Parts of this Ph.D. thesis have been published separately:

[1] A. Visscher, S. Bachmann, C. Schnegelsberg, T. Teuteberg, R. A. Mata, D. Stalke, „Highly selective and sensitive fluorescence detection of $\mathrm{Zn}^{2+}$ and $\mathrm{Cd}^{2+}$ ions by using an acridine sensor", Dalton Trans. 2016, 45, 5689-5699.

[2] S. Bachmann, R. Neufeld, M. Dzemski, D. Stalke, „New external calibration curves (ECCs) for the estimation of molecular weights in various common NMR solvents.", Chem. Eur. J. 2016, 22, 8462-8465.

[3] C. Schnegelsberg, S. Bachmann, M. Kolter, T. Auth, M. John, D. Stalke, K. Koszinowski, "Association and dissociation of Grignard reagents $\mathrm{RMgCl}$ and their turbo variant $\mathrm{RMgCl} \cdot \mathrm{LiCl}$ ", Chem. Eur. J. 2016, 22, 7752-7762.

[4] S. Bachmann, B. Gernert, D. Stalke, „Solution structures of alkali metal cyclopentadienides in THF estimated by ECC-DOSY NMR-spectroscopy (incl. software)", Chem. Commun. 2016, 52, 12861-12864.

Other publications related to this thesis are in preparation:

[5] A. Kreyenschmidt, S. Bachmann, T. Niklas, D. Stalke, „Molecular Weight Prediction of Molecules incorporating Heavier Elements using a Correction Factor for ECC-DOSY", in preparation.

[6] S. Bachmann, T. Niklas, M. John, D. Stalke "Structure elucidation of s-Block organometallics with diffusion NMR", in preparation. 


\section{INTRODUCTION}

\subsection{Organometallic Chemistry}

Many Nobel Prizes have been awarded for the distinct subfield of organometallic chemistry, e.g. to Fischer and Wilkinson in 1973 for their work on metallocenes or to Chauvin, Grubbs and Schrock in 2005 for metal-catalyzed olefin metathesis. This shows the continuous importance of the field and it is therefore not surprising that organometallics, characterized by more or less polar bonds between $\mathrm{M}^{\delta^{+}}$(metal) and $\mathrm{C}^{\delta^{-}}$(carbon), have an innumerable amount of applications, e.g. in catalysis or as single molecular magnets. ${ }^{[7]}$ Organometallic precursor materials incorporate mostly alkali or alkaline earth metals. The understanding of such precursors will be the main focus of this thesis.

\subsubsection{Lithiumorganics}

Lithiumorganics have been of interest for many years as they possess extraordinary reactivities. ${ }^{[8]}$ Concerning this work, they resemble ideal case studies to explain basic aggregational patterns. The first lithiumorganic was reported back in 1917 by Schlenk and Holtz ${ }^{[9]}$ who synthesized methyllithium (MeLi) by transmetalation of dimethylmercury with elemental lithium. Since then the field has become much more diverse and lithiumorganics are nowadays available for a variety of applications, e.g. in pharmaceutical or polymer syntheses. ${ }^{[10]}$ In many cases they are formulated in chemical equations as RLi, indicating just a simple monomeric aggregate. However, it could already be shown over 50 years ago by Dietrich ${ }^{[1]}$ with the publication of the tetrameric aggregation of ethyllithium in the solid state $\left([\mathrm{EtLi}]_{4}\right)$ that this view is too short-sighted. Most lithiumorganics favor forming oligomeric structures in the form of hexamers (e.g. $[n \mathrm{BuLi}]_{6}^{[12]} ;[i \mathrm{PrLi}]_{6}{ }^{[13]}$ ) or tetramers (e.g. $[\mathrm{MeLi}]_{4}{ }^{[14]} ;[\mathrm{EtLi}]_{4}^{[11]}$ ), depending not only on electrostatics but more importantly on differing organic residues and their respective steric demand. These aggregational motifs can in many cases be disaggregated to dimers (e.g. $[n \mathrm{BuLi}(\mathrm{TMEDA})]_{2}{ }^{[15]} ;\left[t \mathrm{BuLi}\left(\mathrm{Et}_{2} \mathrm{O}\right)\right]_{2}{ }^{[12]}$ ) or even monomers (e.g. $[\mathrm{PhLi}(\mathrm{PMDETA})]^{[16]}$ ) by the addition of neutral Lewis bases which supposedly mirror conditions present in solution during syntheses (see Figure 1-1). The principles defining these (dis)aggregational processes have already been reviewed in literature ${ }^{[17]}$ and shall not be discussed further herein.
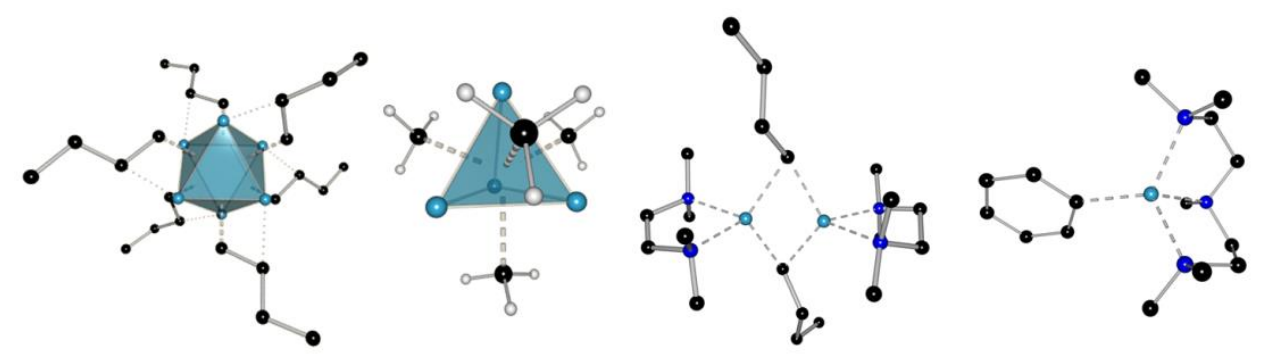

Figure 1-1: Aggregational motifs of lithiumorganics (from left to right: hexameric $[n \mathrm{BuLi}]_{6}{ }^{[12]}$, tetrameric $[\mathrm{MeLi}]_{4}{ }^{[14]}$, dimeric $[n \mathrm{BuLi}(\mathrm{TMEDA})]_{2}{ }^{[15]}$ and monomeric $\left.[\mathrm{PhLi}(\mathrm{PMDETA})]^{[16]}\right)$. Structures were reproduced from the data available in the cited references. 


\subsubsection{Relationship of Structure and Reactivity}

Determination of solid and solution state structures is immensely important to establish a structure-reactivity relationship. As a rule of thumb, lower aggregates of lithiumorganics (e.g. dimers; monomers) are more reactive than higher aggregates (e.g. hexamers; tetramers). This can be underlined by investigations of BuLi: In a recent study, Reich et al. ${ }^{[18]}$ found with the help of rapid injection NMR (RINMR) ${ }^{[19]}$ that dimeric $n \mathrm{BuLi}$ reacts $3.2 \cdot 10^{8}$ times faster with (phenylthio)acetylene than its tetrameric aggregate (see Scheme 1-1). A few decades earlier, the same reactivity trend could already be observed towards other reactants by McGarrity et al., ${ }^{[20]}$ even though exact relative reactivity rates were not determined.

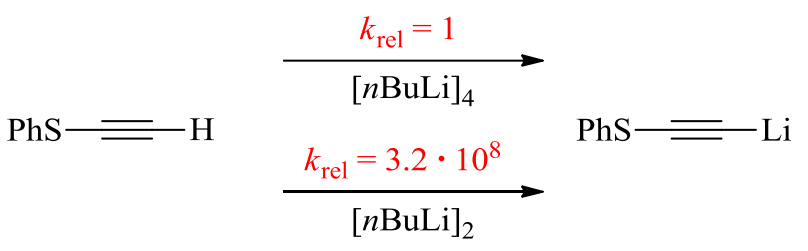

Scheme 1-1: Lithiations of (phenylthio)acetylene with different $n \mathrm{BuLi}$ aggregates show an increased reactivity for the dimeric species compared to the tetrameric $n \mathrm{BuLi}^{[18]}$

Ogle et al. ${ }^{[21]}$ used the same technique to examine different BuLi isomers and derived relative reactivities towards the initiation of the polymerization reaction of styrene in THF. They concluded that the order of reactivity for the isomers is $s \mathrm{BuLi}>t \mathrm{BuLi}>n \mathrm{BuLi}$ for this observed reaction, whereas in hydrocarbon solvents it is $s \mathrm{BuLi}>n \mathrm{BuLi}>t \mathrm{BuLi}$. These reactivity differences are also closely related to the aggregational states of the BuLi isomers. Theoretical calculations of the same study revealed a reactivity trend of $[n \mathrm{BuLi}]_{1}>[s \mathrm{BuLi}]_{1}>[t \mathrm{BuLi}]_{1}>[n \mathrm{BuLi}]_{2}$, where the subscripts 1 and 2 refer to the monomeric and dimeric forms, respectively. ${ }^{[21]}$ All those findings point towards higher reactivities for smaller BuLi aggregates, as was assumed beforehand.

The inherent decrease in selectivity, when going to lower aggregational states, is mentioned by Ebewele: ${ }^{[22]}$ In an anionic polymerization reaction of 1,3-butadiene in unpolar solvents with $n \mathrm{BuLi}$ as initiator a high content of cis-1,4-polybutadiene is produced, whereas in more polar solvents the content of the cis-1,4-polymer decreases significantly which results in a cis-1,4-/ trans-1,4-polybutadiene mixture (see Scheme 1-2).

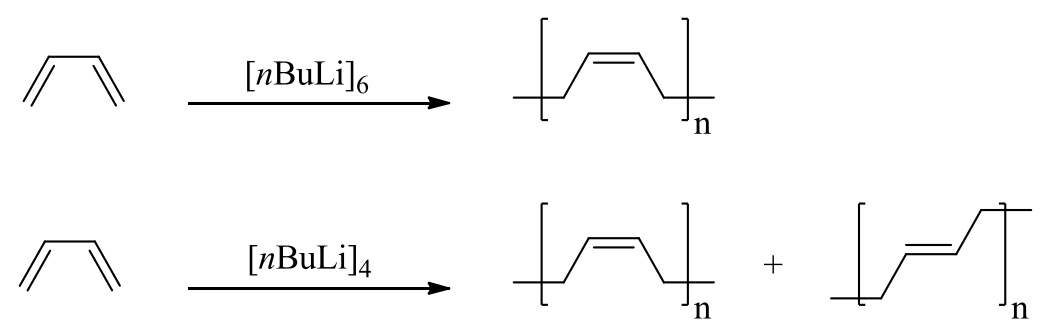

Scheme 1-2: Polymerization reactions of 1,3-butadiene with different $n$ BuLi aggregates show a lower selectivity of these reactions for the smaller tetrameric $n$ BuLi aggregate, when compared to hexameric $n \mathrm{BuLi} .{ }^{[22]}$ 
However, this rule also has its exceptions: In 2003 Zhao and Collum ${ }^{[23]}$ could show for an enolization reaction of 2-methylcyclohexanone with LiHMDS that a dimer-based mechanism using $\mathrm{Et}_{3} \mathrm{~N}$ as co-solvent can lead to a faster reaction than the monomer-based mechanism with the co-solvent THF. Collum ${ }^{[24]}$ argued even earlier that the acceleration of organolithium reactions with strongly donating solvents is not altogether logical since strong solvation, high reactivity and lower aggregates are not necessarily, mutually dependent. There are other examples to support him, e.g. Klumpp et al. ${ }^{[25]}$ showed that some "secondary alkyllithium dimers" [...] "are less reactive towards ethylene than their tetramer counterparts". Even though the rule holds true for $n \mathrm{BuLi}$, these counterexamples show that it is best to fully understand the solvation as well as aggregation of organometallics to access final reactivities.

\subsubsection{Solvent-Separated Ion Pairs (SSIPs)}

While all the structures mentioned in the sections above fall within the confines of the class of contact ion pairs (CIPs), there is another important aggregational motif in organometallic chemistry: Charged solvent-separated ion pairs (SSIPs). ${ }^{[26]}$ The term SSIP is generic and can be used for lithiumorganics, e.g. for $\left[\mathrm{Li}(\mathrm{THF})_{\mathrm{n}}\right]\left[\mathrm{C}\left(\mathrm{SiCH}_{3}\right)_{3}\right]$ reported by Reich et al., ${ }^{[27]}$ or even when no lithium atoms at all are involved. If both, the anionic and cationic particles, contain lithium, they are known as lithium lithiates (e.g. $\left.\left[\mathrm{Li}(\mathrm{THF})_{4}\right]\left[\mathrm{Li}\left(\mathrm{C}\left(\mathrm{SiMe}_{3}\right)_{3}\right)_{2}\right]\right){ }^{[28]}$ SSIPs are often even more reactive and therefore more unstable than their CIP counterparts and it is thus not surprising that the first crystal structure displaying this aggregation was not obtained before 1983: By then Eaborn and Smith et al. ${ }^{[28]}$ crystallized $\left[\mathrm{Li}(\mathrm{THF})_{4}\right]\left[\mathrm{Li}\left(\mathrm{C}\left(\mathrm{SiMe}_{3}\right)_{3}\right)_{2}\right]$ from THF solution. Since then, many other crystal structures of alkali or alkaline earth metal SSIPs have been published. ${ }^{[29]}$ Furthermore, they were studied extensively in solution. ${ }^{[27,30]}$ From all of these studies it can be concluded that SSIPs are formed and retained by strongly donating solvents with a high dielectric constant (see Table 1-1), such as HMPA, DMSO or in some cases even THF.

Table 1-1: Dielectric constants $(\varepsilon)$ of a selection of solvents.

\begin{tabular}{llllllllll}
\hline Solvent & $\mathrm{CH}_{2} \mathrm{Cl}_{2}{ }^{\mathrm{a}}$ & $\mathrm{C}_{6} \mathrm{H}_{12}{ }^{\mathrm{a}}$ & $\mathrm{C}_{6} \mathrm{H}_{6}{ }^{\mathrm{a}}$ & $\mathrm{CHCl}_{3}{ }^{\mathrm{a}}$ & $\mathrm{THF}$ & $\mathrm{HMPA}$ & DMF & DMSO & $\mathrm{H}_{2} \mathrm{O}$ \\
\hline $\boldsymbol{\varepsilon}^{[31]}$ & 1.6 & 2.0 & 2.3 & 4.8 & 7.5 & 31 & 38 & 47 & 79 \\
\hline
\end{tabular}

a) These solvents do not normally lead to SSIP structures.

Moreover, it is important that SSIPs can occur simultaneously with CIPs in solution. The aforementioned $\left[\mathrm{Li}(\mathrm{THF})_{\mathrm{n}}\right]\left[\mathrm{C}\left(\mathrm{SiCH}_{3}\right)_{3}\right]$ was shown to coexist in an equilibrium with the lithium lithiate $\left[\mathrm{Li}(\mathrm{THF})_{\mathrm{n}}\right]\left[\mathrm{Li}\left(\mathrm{C}\left(\mathrm{SiCH}_{3}\right)_{3}\right)_{2}\right]$ and the $\mathrm{CIP}\left[\mathrm{LiC}\left(\mathrm{SiCH}_{3}\right)_{3}\right] .{ }^{[27 a]}$ Additionally, lithium lithiates are also often called triple ions, because their anionic building block consists of a combination of three distinct ions (e.g. $\left[\mathrm{Li}\left(\mathrm{C}\left(\mathrm{SiMe}_{3}\right)_{3}\right)_{2}\right]^{-}$consists of two $\mathrm{C}\left(\mathrm{SiMe}_{3}\right)_{3}{ }^{-}$and one $\left.\mathrm{Li}^{+}\right)$. This portfolio of SSIPs could recently be extended towards the so-called pentuple ion, named by the same principle. ${ }^{\left[29 \mathrm{q}_{3} 32\right]}$ In conclusion, most organometallic structures can be classified as either SSIPs or CIPs and their reactivities also depend in many cases on these basic aggregational concepts. 


\subsubsection{Alkali Metal Cyclopentadienides (CpMs)}

Another important class of compounds in organometallic chemistry are alkali metal cyclopentadienides (CpMs) and their derivatives which have been reviewed in literature $\mathrm{e}^{[33]}$ extensively.

\subsubsection{A Short Historical Background}

A historical landmark of cyclopentadienide chemistry is the synthesis and structure determination of bis(cyclopentadienyl)iron(II), commonly known as ferrocene, which expedited the organometallic chemistry of the transition metals. ${ }^{[34]}$ However, ferrocene was discovered over half a century later than sodium cyclopentadienide $(\mathrm{CpNa})$ which was already synthesized in situ by Thiele $e^{[35]}$ in 1900, even though he was not able to isolate it. However, a year later Thiele ${ }^{[36]}$ was able to synthesize and isolate potassium cyclopentadienide ( $\mathrm{CpK}$ ) which was the first purely isolated organometallic compound featuring an alkali metal. This all took place shortly after the isolation of cyclopentadiene itself which is achieved by the reversible Diels-Alder dimerization reaction of dicyclopentadiene (DCp), even though this reaction behavior was puzzling at those times. ${ }^{\left[{ }^{[7]}\right.} \mathrm{A}$ few years after the discovery of ferrocene, Fischer et al. ${ }^{[38]}$ and Ziegler et al. ${ }^{[39]}$ independently isolated pure $\mathrm{CpNa}$ from xylene solution which made CpMs more accessible. Both studies point out that this had not been possible for Thiele due to his choice of solvent (benzene) and its lower boiling point.

\subsubsection{Practical Applications}

CpMs are highly relevant precursors for a variety of different sandwich or half-sandwich d-block organometallics which can be synthesized through either transmetalation or salt elimination (see Scheme 1-3). ${ }^{[40]}$

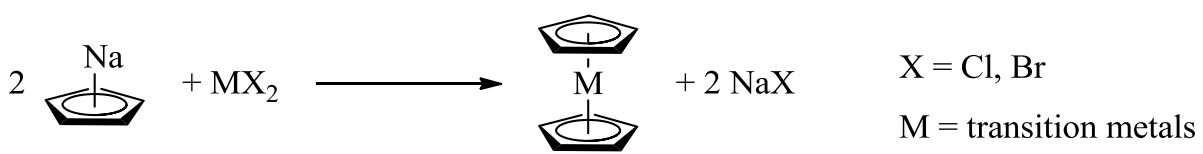

Scheme 1-3: General synthesis of transition metal metallocenes using CpNa as starting material. ${ }^{[4]}$

Such metallocenes, whereas it is debateable, if the term includes only sandwich or also halfsandwich complexes, ${ }^{[33 e]}$ and their derivatives can be used in the catalysis of polymerization reactions of alkenes. Especially the polymerization of propene by homogeneous Kaminsky-catalysts is of major importance in this regard. These were discovered in 1980 by Kaminsky et al. ${ }^{[42]}$ and consist of zirconium, hafnium or titanium sandwich complexes which may also contain bigger ligand systems based on indenides or fluorenides (see Scheme 1-4) in combination with methylaluminoxane (MAO). ${ }^{[43]}$ 

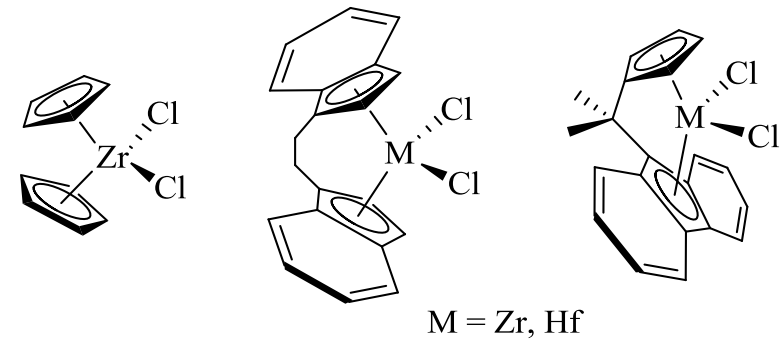

Scheme 1-4: Small selection of metallocene-type Kaminsky-catalysts which can be used for polymerization reactions. ${ }^{[43 c]}$

Different catalysts lead selectively to a-, syndio- or isotactic polymers and can be up to 100 times as effective as their close relatives, the Ziegler-Natta catalysts. ${ }^{[43 c]}$ There are also many other syntheses, wherein complexes incorporating the cyclopentadienide ligand are of use, e.g. the synthesis of isoindolones ${ }^{[44]}$ or of fullerene-ferrocene-hybrids. ${ }^{[45]}$ Recently, Cramer et al. ${ }^{[46]}$ have also been using chiral cyclopentadienide ligands for asymmetric C-H functionalization. Additionally, there are some metallocene dihalides which exhibit anti-tumor properties, although none have proceeded far in clinical trials. ${ }^{[4]}$

\subsubsection{Properties and Bonding}

CpMs are very salt-like which can be underlined by their high melting points und low volatilities. ${ }^{[33,48]}$ Due to this it is not surprising that their solubility in hydrocarbons and ethers (except THF) are quite poor which made structure elucidation, especially crystal structure determination of the donor-free compounds, challenging.

Some first analytical results concerning the bonding of CpMs have been gathered in the $60 \mathrm{~s}$ and $70 \mathrm{~s}$ by $\mathrm{IR}^{[49]}$ and NMR measurements ${ }^{[50]}$ which indicated that the metal cation and $\mathrm{Cp}^{-}$are $\pi$-bonded along its fivefold axis ( $\eta^{5}$-coordination) in solution. Those findings already showed that the $\sigma$-bonded complex of CpLi, i.e. 2-lithio-cyclopentadiene, is unlikely which has also been confirmed by simple ab initio calculations (RHF/6-31+ $\mathrm{G}^{*}$ ) that result in a $49.3 \mathrm{kcal} / \mathrm{mol}$ difference in favor of the $\eta^{5}$ - $\pi$-bonded complex. ${ }^{[33 \mathrm{e}]}$ In principle, the alkali metal could be attached to the $\mathrm{Cp}^{-}$at several positions, ranging from $\eta^{1}$ - to $\eta^{5}$-coordination. However, the hapticity of CpLi was analyzed by means of a theoretical haptotropic search which again emphasized the preference of the $\eta^{5}$-coordination motif (see Figure 1-2). ${ }^{[33 e]}$

Still, Harder ${ }^{[33 e]}$ suggests that minor deviations from a perfect $\eta^{5}$-coordination are common due to the rather flat minimum of the energy curve. The importance of electrostatics on the bonding between alkali metal and cyclopentadienide was explained in an extensive theoretical study by Rayon and Frenking ${ }^{[51]}$ in 2002. 

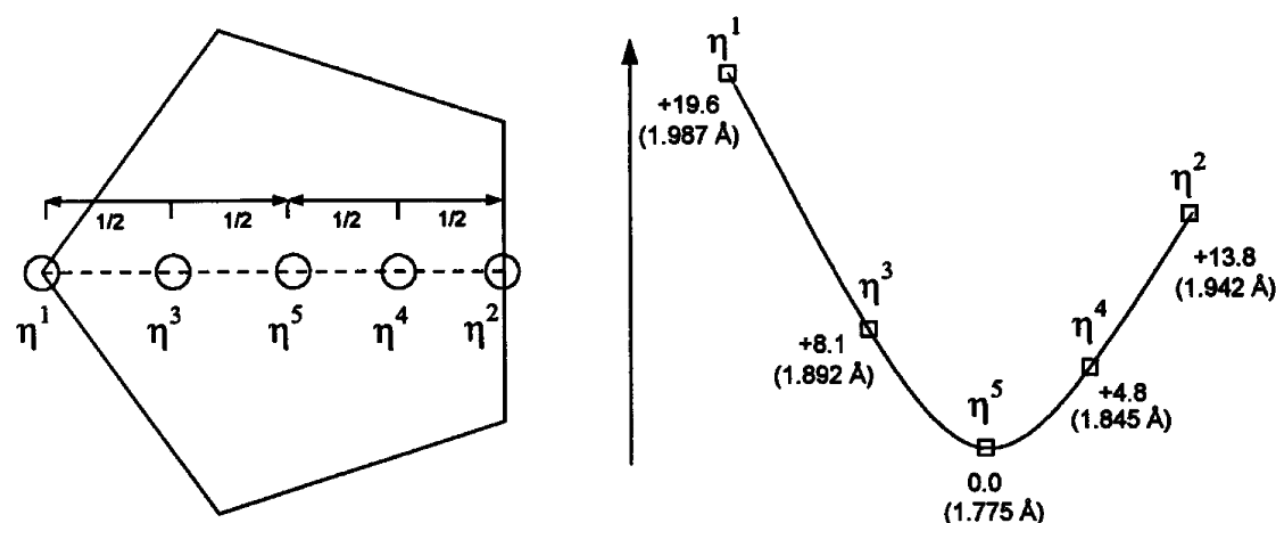

Figure 1-2: Results of a haptotropic search of CpLi $\left(M P 2 / 6-31+\mathrm{G}^{*}\right)$. With decreasing hapticity the energy and distance of lithium to $\mathrm{Cp}^{-}$increase, while lithium is shifted over the Cp-plane. Reprinted from reference [33e]. Copyright () 1998 Elsevier.

\subsubsection{Aggregation of CpMs in the Solid State and in Solution}

The first crystal structure of an underivatized CpM was published in the late $70 \mathrm{~s}$ by Wade et al. ${ }^{[52]}$ with $[\mathrm{CpNa}(\mathrm{TMEDA})]_{\infty}$ which features a polymeric CIP with attached donating Lewis bases. Over the years, other crystal structures of CpMs were presented that entailed similar donor-coordinated polymeric chains. ${ }^{[53]}$ Still, it was not until over 20 years later that structures of donor-free CpMs were published: By then Olbrich et al. ${ }^{[54]}$ used powder diffraction to provide the solid state structures of $\mathrm{CpLi}, \mathrm{CpNa}$ and $\mathrm{CpK}$. These molecules also form CIPs arranged into polymers that are linear and isomorphous for $\mathrm{CpLi}$ and $\mathrm{CpNa}$, whereas for $\mathrm{CpK}$ and the higher homologues bent structures could be observed (see Figure 1-3; for CpRb there are ambivalent structures). ${ }^{[54-55]}$

$$
-|-\mathrm{M}-|-\mathrm{M}-\mid-\mathrm{M}-\mathrm{M}-\mathrm{M}
$$

Figure 1-3: Donor-free aggregation of CpMs (also known for methyl- or silyl-substituted Cp-moieties, see literature mentioned above).

Polymeric donor-free structures are also known for the methylated derivatives (i.e. methylcyclopentadienides $(\mathrm{CpMe})$ or pentamethylcyclopentadienides $\left(\mathrm{Cp}^{*}\right)$ ) and silyl-substituted $\mathrm{Cp}$ systems. ${ }^{[56]}$ While silyl substituents stabilize the cyclopentadienide carbanion, alkyl groups have the opposite effect. Still, alkyl-substituted ring systems are widely used to fine-tune reactivities of the desired products or increase the steric demand. 
In 1983, Jutzi et al. ${ }^{[57]}$ published the first single crystal structures featuring solvated monomeric aggregates $\left(\left[\left(\mathrm{Me}_{3} \mathrm{Si}\right)_{3} \mathrm{CpLi}\right.\right.$ (Base) $]$ with Base $=$ TMEDA, PMDETA or chinuclidin $)$. They and others derivatized the cyclopentadienide with trimethylsilyl-substituents to increase its solubility. ${ }^{[58]}$ Today, many crystal structures are known with monomeric ${ }^{\mathrm{a}}$ or polymeric aggregation, whilst containing additional donor bases (see Figure 1-4). ${ }^{[52-53,57-59]}$ Monomeric aggregation is often achieved by crown ethers or other strongly donating tri-, bi- or monodentate ligands. While monomeric aggregates represent the complete disaggregation of the polymeric chain, in a few cases oligomers could also be observed: ${ }^{[60]}$ In 2011, two oligomeric structures of nonderivatized CpMs were reported by Stalke et al. ${ }^{[290]}$ who found dimeric $\left[(\mathrm{CpLi})_{2}\left(\mathrm{NH}_{3}\right)_{3}\right]$ and Klett et al. ${ }^{[61]}$ who reported tetrameric $[(\mu-\mathrm{Cp}) \operatorname{Li}(\mu-\mathrm{TMP}) \mathrm{Li}]_{4}$.
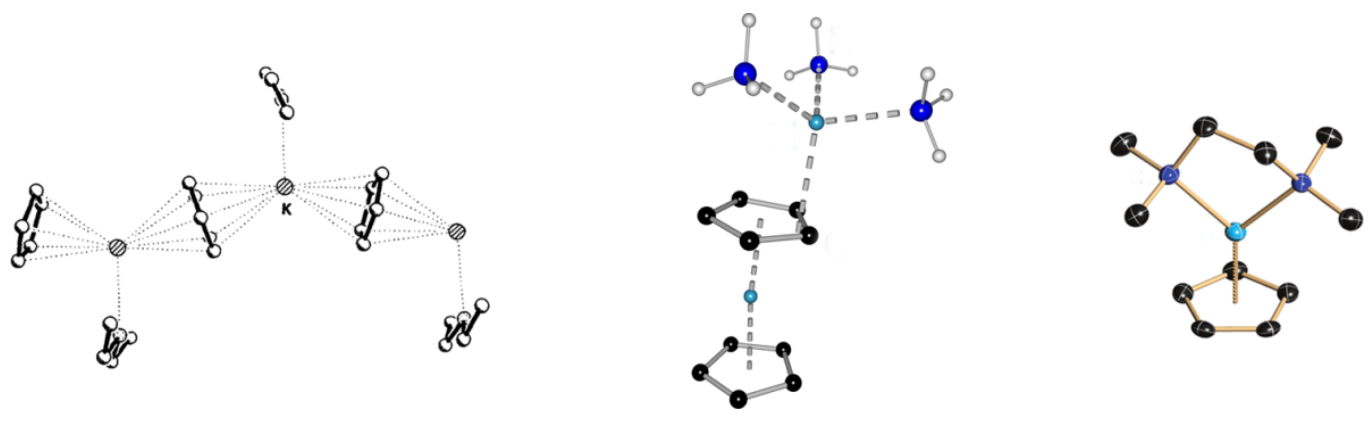

Figure 1-4: Selection of solid state structures of CpMs, illustrating different aggregational possibilities, when incorporating donating solvents. (from left to right: polymeric $\left[\mathrm{CpK}\left(\mathrm{Et}_{2} \mathrm{O}\right)\right]_{\infty}{ }_{\infty}^{[53 \mathrm{a}]}$, dimeric $\left[(\mathrm{CpLi})_{2}\left(\mathrm{NH}_{3}\right)_{3}\right]^{\left[29 \mathrm{O}^{\circ}\right.}$ and monomeric [CpLi(TMEDA)] $\left.{ }^{[59 n]}\right)$ Structures were reprinted from references [53a], [59n] and [29o]. Copyrights ${ }^{\circ} 1996$ Elsevier and (2) 2010, 2011 American Chemical Society.

Lastly, concerning CpM aggregational states, there are also charged species known (see Figure 1-5). The most interesting one is probably the smallest metallocene possible, the lithocene anion $\left(\left[\mathrm{Cp}_{2} \mathrm{Li}\right]^{-}\right)$. It was first crystallized in 1994 by Harder and $\operatorname{Prosenc}^{[62]}$ as $\left[\mathrm{Ph}_{4} \mathrm{P}\right]\left[\mathrm{Cp}_{2} \mathrm{Li}\right]$. A year later, Mews et al. ${ }^{[63]}$ were able to exchange the counter-ion for tris(dimethylamino)sulfonium $\left(\left[\left(\mathrm{Me}_{2} \mathrm{~N}\right)_{3} \mathrm{~S}\right]^{+}\right)$and crystallized not only $\left[\left(\mathrm{Me}_{2} \mathrm{~N}\right)_{3} \mathrm{~S}\right]\left[\mathrm{Cp}_{2} \mathrm{Li}\right]$, but also the sodium analogue (sodocene). Harder et al. ${ }^{[64]}$ reported another sodocene structure a year later $\left(\left[\mathrm{Ph}_{4} \mathrm{P}\right]\left[\mathrm{Cp}{ }_{2} \mathrm{Na}\right]\right)$. Moreover, a silylated lithocene structure was published by Westerhausen et al. ${ }^{[65]}$ $\left(\left[\mathrm{Li}(\mathrm{THF})_{4}\right]\left[\left(\left(\mathrm{Me} \mathrm{Si}_{2} \mathrm{Cp}\right)_{2} \mathrm{Li}\right]\right)\right.$ which can also be classified as a lithium lithiate.

The possibility of the existence of lithocene in solution has been investigated as well. In 1990, Paquette et al. ${ }^{[66]}$ studied CpLi in THF via NMR spectroscopy and found out that there might exist an equilibrium between a monomeric CIP and the lithocene anion. In their study, they focused primarily on lithium isodicyclopentadienide and could prove for this the existence of a monomerdimer equilibrium in THF at low temperatures (the dimer being a sandwich type aggregate). ${ }^{[6]}$ For CpLi they proposed a similar behavior, even though exchange rates seem to be much faster and they were not able to "tell whether the monomer-dimer equilibrium [...] is shifted to either side at room temperature". ${ }^{[66]}$ In the same year, Brandsma et al. ${ }^{[67]}$ used cryoscopy to examine CpLi within

${ }^{a}$ Monomeric CpM aggregates are in some cases called "piano stool” or half-sandwich complexes. 
ammonia and proposed that CpLi does not form simple monomeric [CpLi] therein, whereas higher aggregates, including charged species, like lithocene, are likely. This is also hinted at by findings of Stalke et al. ${ }^{[290]}$ who could show an involvement of lithocene type aggregates within a crystal structure (middle of Figure 1-4; $\left.\left[(\mathrm{CpLi})_{2}\left(\mathrm{NH}_{3}\right)_{3}\right]\right)$ which was crystallized from ammonia and might be preserved in solution as well.

Stalke et al.$^{[20]}$ also crystallized another unprecedented aggregate utilizing a higher concentration of ammonia: A SSIP consisting of $\mathrm{Cp}^{-}$anions with solvent-coordinated $\left[\mathrm{Li}\left(\mathrm{NH}_{3}\right)_{4}\right]^{+}$cations (see Figure 1-5). A handful of such SSIPs have been published over the years, but $\mathrm{Cp}^{-}$anions could, in contrast to the above mentioned case, only be stabilized by sterically demanding cations. ${ }^{[33 b, 63]}$ Aside from metallocene type structures, like the lithocene (a), cationic inverse sandwich complexes $^{[68]}(\mathrm{b})$ as well as a caesocene triple-decker structure ${ }^{[69]}$ (c) were reported which conclude the range of charged species known for CpMs.

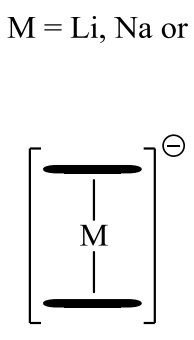

(a)

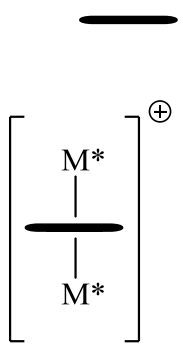

(b)

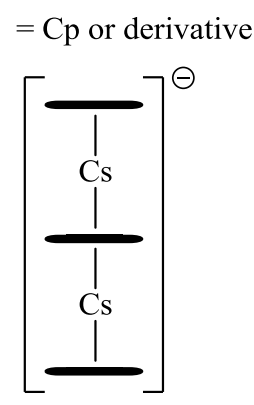

(c)

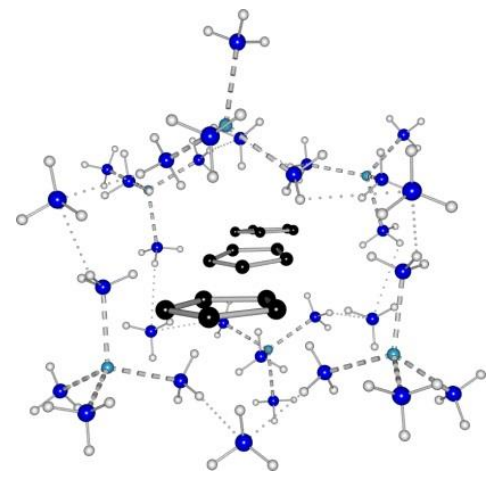

Figure 1-5: Selection of charged species known from crystal structure analysis (from left to right: metallocene-type sandwich complexes (a), inverse sandwich complexes (b), a caesocene triple-decker (c) (donating solvent molecules have been omitted for clarity) and an exemplary SSIP ([Li(NH3)4][Cp]). The SSIP structure (right) was reprinted from reference [29o]. Copyright $\odot 2010$ American Chemical Society.

\subsection{Nuclear Magnetic Resonance (NMR) Spectroscopy}

Even though most structures discussed above have been solved by single crystal or powder X-ray diffraction, and in recent years many advances were made in this field to improve structure determination of volatile, air- or moisture-sensitive compounds and the handling of crystals at lower temperatures, it is still only a first step towards understanding a molecule's reactivity. ${ }^{[70]}$ While a crystal structure can mostly be viewed as unambiguous, solution state structures are often complex equilibria of multiple co-existing aggregates. Since reactions are mostly carried out in solutions, it is therefore imperative to understand these equilibria and the individual aggregates thereof. While there are other methods available to observe and afterwards interpret the constitution of a molecule in solution (e.g. ultraviolet-visible (UV-VIS) or infrared (IR) spectroscopy, cryoscopy, ebulliometry), NMR spectroscopy has been on the rise in the last decades and can in many ways go beyond or be combined with other methodologies. Nowadays, advanced high-resolution NMR methods exist or are being developed which can be used to monitor reactions 
or even determine a molecule's stereochemistry (e.g. by Niklas et al. ${ }^{[71]}$ ). Furthermore, many other methods published in the last decades (e.g. correlation spectroscopy (COSY), heteronuclear single quantum correlation (HSQC), heteronuclear multiple bond correlation (HMBC) and many more) added to the NMR spectroscopisc's repertoire. The basics and theoretical background of NMR spectroscopy as well as most routinely used $1 \mathrm{D}$ and $2 \mathrm{D}$ methods have been reviewed extensively in literature ${ }^{[72]}$ and will not be discussed in this work. ${ }^{\mathrm{a}}$

\subsubsection{Heteronuclear Overhauser Enhancement Spectroscopy (HOESY)}

Apart from routine experiments or diffusion NMR techniques which will be elucidated in the following sections, heteronuclear Overhauser enhancement spectroscopy (HOESY) is repeatedly utilized throughout this work. This type of experiment was introduced independently by Levy et al. ${ }^{[73]}$ and Rinaldi ${ }^{[74]}$ in 1983. It is derived from nuclear Overhauser enhancement spectroscopy (NOESY) which visualizes homonuclear dipole-dipole interactions through space using the nuclear Overhauser effect (NOE). The HOESY technique, in contrast to NOESY, aims at heteronuclear couplings and is nowadays often used in lithium chemistry to observe short ${ }^{6} \mathrm{Li}^{-}{ }^{1} \mathrm{H}$ or ${ }^{7} \mathrm{Li}^{-1} \mathrm{H}$ distances (up to $4 \AA$ ). ${ }^{[75]}$ This allows discrimination between different aggregational states (CIP vs. SSIP), since dipole-dipole interactions to solvents or ligandsystems can be interpreted. If used qualitatively, it is intuitive: If a crosspeak is observed, the coupling nuclei have to be in close proximity to each other. Furthermore, the higher the intensity of the crosspeak the closer are the nuclei, since the crosspeaks intensity is proportional to $\frac{1}{r^{6}}$. Hence, even quantitative distance calculations are possible. ${ }^{[76]}$

Von Ragué Schleyer et al. ${ }^{[75]}$ were the first to use HOESY in organolithium chemistry. ${ }^{[77]}$ They showed that ${ }^{6} \mathrm{Li}-{ }^{1} \mathrm{H}$ couplings can effortlessly be observed at natural abundance. This is possible since ${ }^{6} \mathrm{Li}$ has the smallest quadrupolar moment known and behaves like a spin-1/2 nucleus (see Table 1-2).

Table 1-2: Quadrupole moments $(Q)$ of alkali metal nuclei.

\begin{tabular}{llllllllll}
\hline Nucleus & ${ }^{6} \mathrm{Li}$ & ${ }^{7} \mathrm{Li}$ & ${ }^{23} \mathrm{Na}$ & ${ }^{39} \mathrm{~K}$ & ${ }^{39} \mathrm{~K}$ & ${ }^{41} \mathrm{~K}$ & ${ }^{85} \mathrm{Rb}$ & ${ }^{87} \mathrm{Rb}$ & ${ }^{133} \mathrm{Cs}$ \\
\hline $\boldsymbol{Q}^{[78]}\left[\mathbf{f m}^{2}\right]$ & -0.0808 & -4.01 & 10.4 & 5.85 & -7.3 & 7.11 & 27.6 & 13.35 & -0.343 \\
\hline
\end{tabular}

Interestingly enough, ${ }^{7} \mathrm{Li},{ }_{1}^{1} \mathrm{H}$-HOESY experiments are also possible, even though its quadrupole moment is relatively large (see Table 1-2). ${ }^{133} \mathrm{Cs},{ }^{1} \mathrm{H}$-HOESY has also been applied in a few cases, but for it to be successful, $T_{1}$ relaxation times have to be higher than $0.1 \mathrm{~s} .{ }^{[79]}$ For other alkali metals HOESY experiments are unknown, as their quadrupole moments are even larger (see Table 1-2) which enables quadrupolar relaxation and reduces the NOE needed to observe interactions through space.

${ }^{\text {a }}$ Information on alkali metal NMR spectroscopy is given as deemed appropriate. 


\subsubsection{Overview of Diffusion NMR}

Aside from the above-mentioned approaches, diffusion NMR experiments play a fundamental role in solving aggregational phenomena. Through such experiments diffusion coefficients $(D)$ of molecules can be determined and afterwards correlated to their sizes or weights. The theoretical background as well as related experiments have been reviewed extensively in literature ${ }^{[80]}$ and shall only be discussed shortly herein. The basics of the methodology were already developed in the mid-1960s by Stejskal and Tanner ${ }^{[81]}$ who were looking for a way to reliably determine the self-diffusion coefficient of solutes. The result was the pulsed-field gradient spin-echo (PFG-SE) pulse sequence (see Figure 1-6).

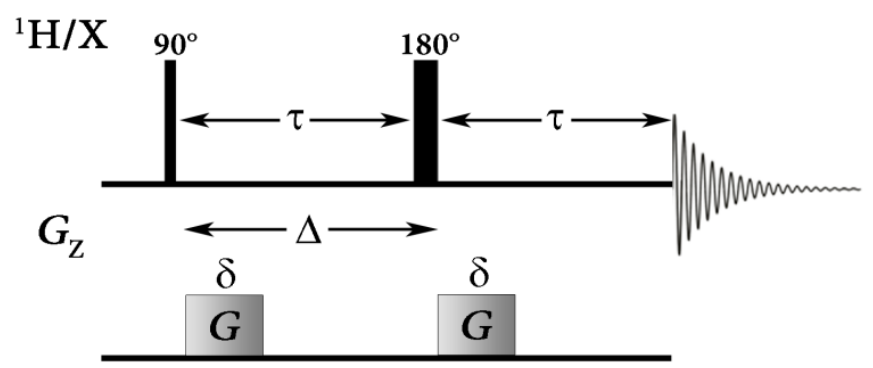

Figure 1-6: Pulse sequence of the PFG-SE experiment. ${ }^{[81]}$

It incorporates the Hahn spin-echo sequence ${ }^{[82]}$ (consisting of a $90^{\circ}$ and a $180^{\circ}$ pulse) which is used to refocus the chemical shift evolution. This way, the detected signal is only attenuated by its spin-spin relaxation $\left(T_{2}\right)$ during the experiment $(2 \tau)$. Additionally, pulsed-field gradients are employed to encode the physical location of a spin. Molecules diffuse through random translational motion (Brownian motion) driven by the thermal energy of the system. This motion changes the physical location of a molecule (depending on its individual diffusion coefficient) and through the imposed field gradient also the local field it experiences. Due to this change in local field, magnetization is not completely refocused after the completion of the spin-echo sequence.

Varying the diffusion delay (time the molecule can diffuse; $\Delta$ ), the length of the gradient pulses $(\delta)$ or their strength $(G)$ enables the characterization and differentiation of diffusion rates (see Figure 1-7).

\footnotetext{
a In literature sometimes PGSE.

${ }^{\mathrm{b}}$ Varying the gradient strength is preferred as longer diffusion times increase the loss of magnetization due to $T_{2}$ relaxation.
} 


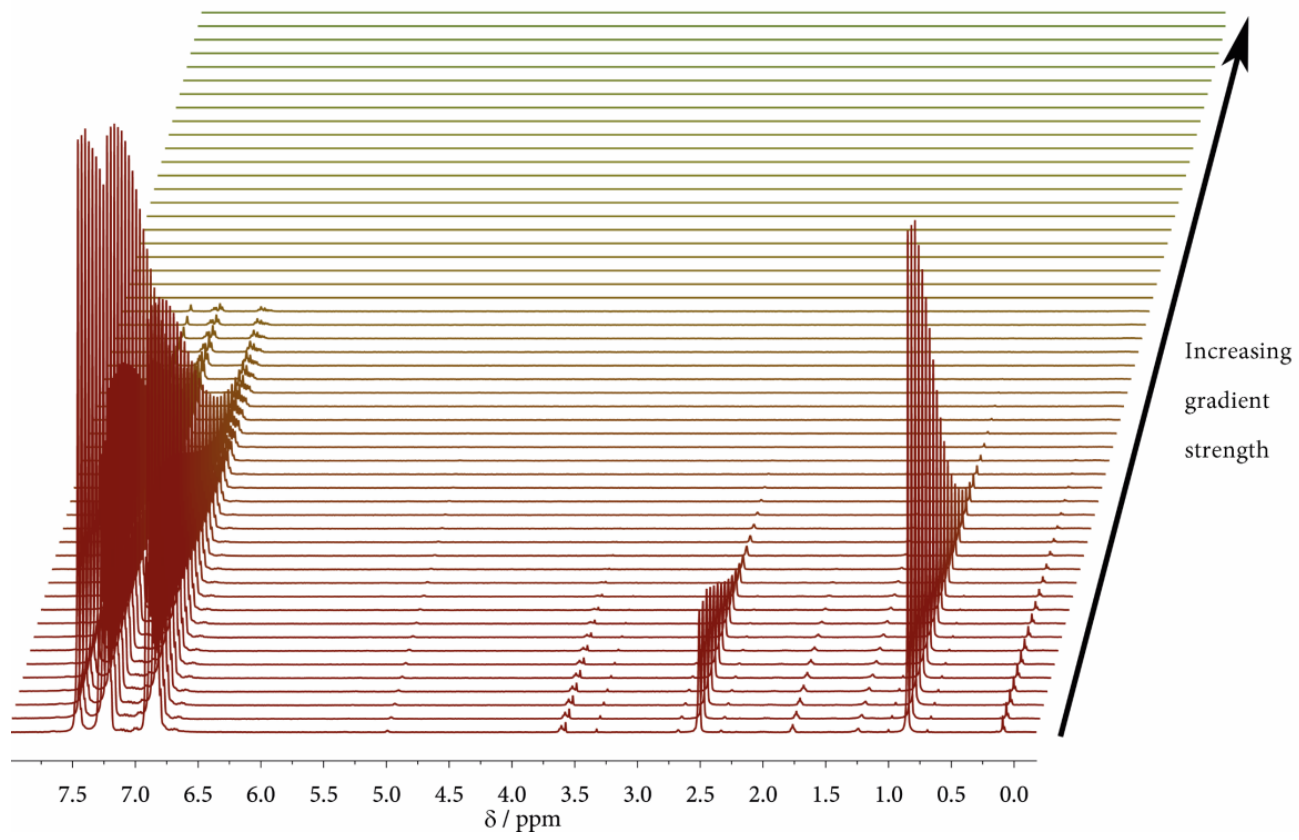

Figure 1-7: ${ }^{1} \mathrm{H}$ signal attenuation due to the continuous increase in gradient strength $(G)$ in PFG-SE-based experiments.

\subsubsection{Calculating Diffusion Coefficients}

The signal intensity after a PFG-SE experiment $\left(I_{\mathrm{G}}\right)$ can be expressed by the Stejskal-Tanner equation (1-1) and is thereby correlated directly to the desired diffusion coefficient.

$$
I_{\mathrm{G}}=I_{0} \exp \left(-\frac{2 \tau}{T_{2}}\right) \exp \left(-(\gamma \delta G)^{2} D\left(\Delta-\frac{\delta}{3}\right)\right)
$$

Here $I_{0}$ represents the signal intensity recorded after the first $90^{\circ}$ pulse before the application of the field gradient and spin echo, while $G$ is the gradient strength, $D$ the diffusion coefficient, $\gamma$ the gyromagnetic ratio of the observed nuclei, $T_{2}$ the transverse relaxation rate constant and $\tau, \delta$ and $\Delta$ delays that are shown in Figure 1-6.

Variation of $G$ leads to a Gaussian decay profile for $I_{\mathrm{G}}$ that can be fitted according to equation (1-1). ${ }^{\mathrm{a}}$ The signal attenuation should be neither too fast nor too slow as to consider all recorded points and get information as reliable as possible (see Figure 1-8).

Other possibilities to determine diffusion coefficients are to plot $I_{\mathrm{G}}$ against $G^{2}$ which results in an exponential profile or $\ln \left(I_{\mathrm{G}}\right)$ against $G^{2}$ which can be fitted linearly. These options are also available in modern software applications, e.g. Topspin 3.1. To obtain the diffusion coefficients of individual components of a mixture, their resonances should be clearly separated. ${ }^{\mathrm{b}}$ If, however, multiple signals overlap, the signal attenuation is comprised of a multiexponential decay.

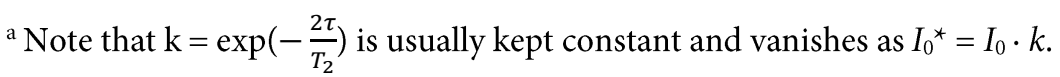

${ }^{\mathrm{b}}$ Baseline distortions should also be avoided for a precise signal intensity acquisition.
} 


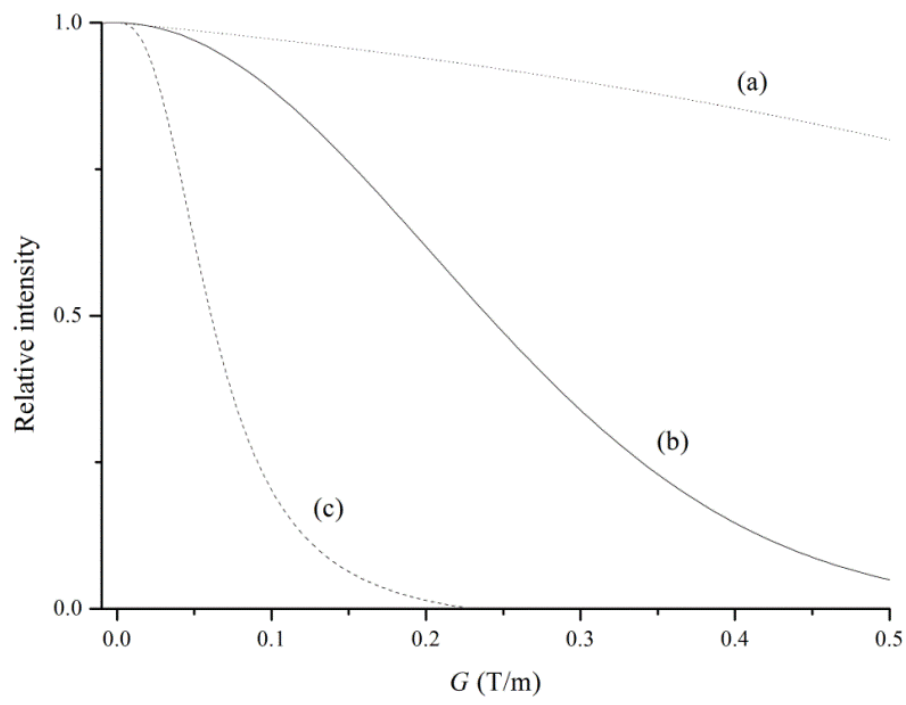

Figure 1-8: Qualitative Gaussian signal intensity decay profiles ( $I_{\mathrm{G}}$ vs. G). Signal attenuation should not be too slow (a) or too fast (c). In an optimal decay profile (b) all points can contribute to the determination of $D .{ }^{[80 e]}$

\subsubsection{Advances in Diffusion NMR}

Modern NMR spectrometers are usually equipped with probe heads with the capability to generate pulsed-field gradients, and therefore diffusion NMR has become routinely accessible. Furthermore, there are improved pulse sequences available (e.g. pulsed field gradient stimulated-echo $(\mathrm{PFG}-\mathrm{STE})^{[83]}$, bipolar pulse pairs with longitudinal-eddy-current-delay (BPP-LED) ${ }^{[84]}$ or double-stimulated-echo (D-STE) ${ }^{[85]}$ ) that reduce experimental times, magnetization loss through relaxation, spectral (eddy-current ${ }^{\mathrm{a}}$ ) distortions or the effect of convection.

Another technique that is gaining popularity and works well in combination with various NMR techniques is called pure-shift NMR. ${ }^{[8]}$ This technique entails homo nuclear decoupling and thus reduces signal overlap which is crucial for diffusion NMR. In 2014, Maddaluno and Oulyadi et al. ${ }^{[87]}$ showed the effectiveness of the combination of the two techniques (diffusion and pure-shift NMR) on model $n$ BuLi aggregates in THF.

\subsubsection{Diffusion-Ordered Spectroscopy (DOSY)}

While 1D NMR experiments provide chemical shifts $(\delta)$ and scalar homo- or heteronuclear coupling constants $(J)$ as basic and resilient parameters to judge a sample's composition, it was the introduction of a $2^{\text {nd }}$ dimension which enabled easier access to their relations. In 1992, Johnson and Morris ${ }^{[8]}$ introduced a new representation for diffusion experiments which was a (pseudo) 2D arrangement they named diffusion-ordered spectroscopy (DOSY). Using DOSY, different components within a mixture are visually separated by their diffusion coefficients which are still

\footnotetext{
${ }^{a}$ Swirling (eddy) currents are produced during rapid changes in the magnetic field (e.g. gradients) induced in
} conducting materials (mostly any metallic component in the surrounding NMR spectrometer). 
being correlated to their chemical shift information (see Figure 1-9). The methodology has gained huge popularity since its publication and is frequently called "NMR chromatography". ${ }^{a 9]}$

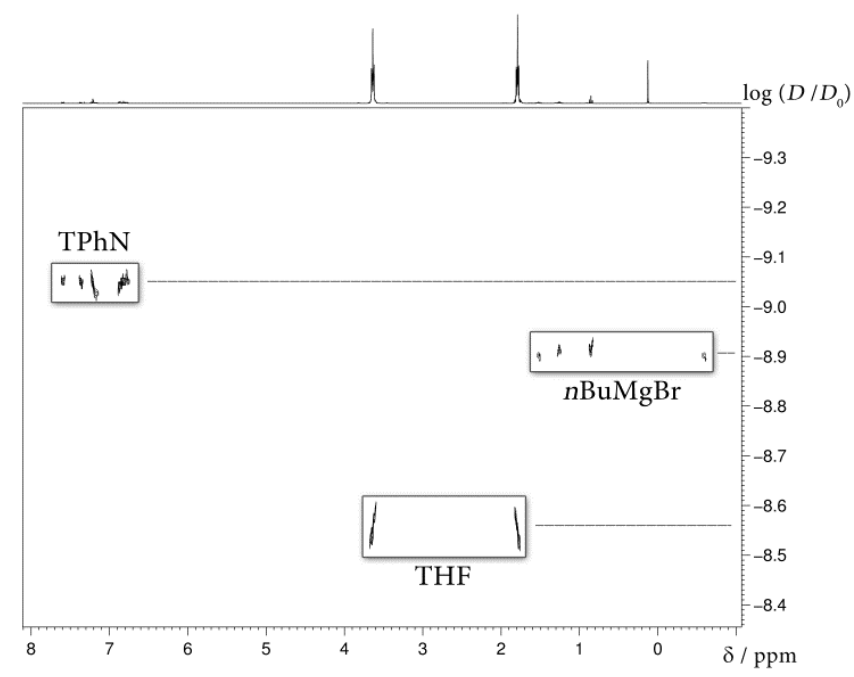

Figure 1-9: Pseudo 2D ${ }^{1} \mathrm{H}$-DOSY spectrum of $n$-butylmagnesium bromide $(n \mathrm{BuMgBr})$ and the internal reference 1,2,3,4-tetraphenylnaphthalene $(\mathrm{TPhN})$ in THF- $d_{8}$. Signals corresponding to the same compound have the same diffusion coefficient and appear in the same row of the spectrum. $\left(D_{0}=1 \mathrm{~m}^{2} / \mathrm{s}\right)$

In organometallic chemistry, DOSY has been used in the last few decades to analyze a number of transition metal complexes. ${ }^{[90]}$ Additionally, the work of Williard et al. ${ }^{[91]}$ should be emphasized, as they have been focusing heavily on lithiumorganics (e.g. they proved the tetramer-dimer equilibrium of $n \mathrm{BuLi}$ in THF using DOSY). These studies also focused heavily on heteronuclear DOSY experiments. Even though heteronuclei often suffer from lower sensitivities, due to their higher chemical shift ranges (e.g. for ${ }^{13} \mathrm{C}$ ) their employment can reduce signal overlap. Moreover, such DOSY analyses can be used to prove coordination behavior (e.g. for $\left.{ }^{7} \mathrm{Li}\right)$. However, it does not work for nuclei with short $T_{2}$ relaxation times.

Recent developments expand the DOSY methodology towards the $3^{\text {rd }}$ dimension to correlate more vital information to the diffusivity of aggregates. ${ }^{[55,92]}$

\subsubsection{Molecular Sizes and Weights from Diffusion Coefficients}

A key interest of chemists lies within the correlation of self-diffusion coefficients $(D)$ to molecular sizes or weights to deduce either aggregational states or interpret other diffusion phenomena (e.g. host-guest interactions). ${ }^{[93]}$ The relationship of the diffusion coefficients to the size of a molecule can most easily be shown by the Stokes-Einstein equation (1-2): ${ }^{[94]}$

$$
D=\frac{k_{\mathrm{b}} T}{6 \pi \eta r_{\mathrm{S}}}
$$

${ }^{a}$ Chromatography is usually used for diffusion experiments supported by stationary phases. 
Here, $r_{\mathrm{S}}$ represents the Stokes-radius which is the radius of a hard sphere that diffuses at the same rate as the observed solute. $k_{\mathrm{b}}$ is the Boltzmann constant, $T$ the absolute temperature and $\eta$ the solvent viscosity at the respective temperature.

However, this equation is only valid for spherical molecules that are much larger than the solvent. While it may be acceptable to make size estimations in a qualitative way, further steps have to be taken to receive more quantitative results, especially for molecules that match or fall below the size of the solvent. Early on, it was proposed to change the frictional coefficient (denominator) " $6 \pi \eta r_{\mathrm{S}}$ " (stick-boundary condition) to " $4 \pi \eta r_{\mathrm{S}}$ " (slip-boundary condition). ${ }^{[94]}$ Later, it was argued that instead of a fixed value, a correctional factor $(c)$ should be introduced that depends on the ratio of the radii of solvent $\left(r_{\text {solv }}\right)$ and solute $\left(r_{\mathrm{s}}\right)$. Additionally, a second correctional term $\left(f_{\mathrm{s}}\right)$ needs to be added to take differing solute geometries/shapes into account. The combination of both correctional factors leads to equation (1-3).

$$
D=\frac{k_{\mathrm{b}} T}{c\left(r_{\mathrm{solv}}, r_{\mathrm{S}}\right) \cdot f_{\mathrm{s}}(a, b) \cdot \pi \eta r_{\mathrm{S}}}
$$

Multiple attempts to express $c$ as function of $r_{\text {solv }} / r_{\mathrm{S}}$ resulted in equation (1-4) by Gierer and Wirtz ${ }^{[95]}$ attained by microfrictional theory, and equation (1-5) by Chen and Chen ${ }^{[96]}$ who used a semi-empirical approach. Both equations result in a numerical factor of 6 for bigger solutes, while Chen's approach reaches this value faster (see Figure 1-10). Still, they drastically improve the model for solutes that have a similar size as the solvent.

$$
\begin{aligned}
& c=\frac{6}{1.5 \cdot\left(\frac{r_{\mathrm{solv}}}{r_{\mathrm{S}}}\right)+\frac{1}{1+\left(\frac{r_{\mathrm{solv}}}{r_{\mathrm{S}}}\right)}} \\
& c=\frac{6}{1+0.695 \cdot\left(\frac{r_{\mathrm{solv}}}{r_{\mathrm{S}}}\right)^{2.234}}
\end{aligned}
$$

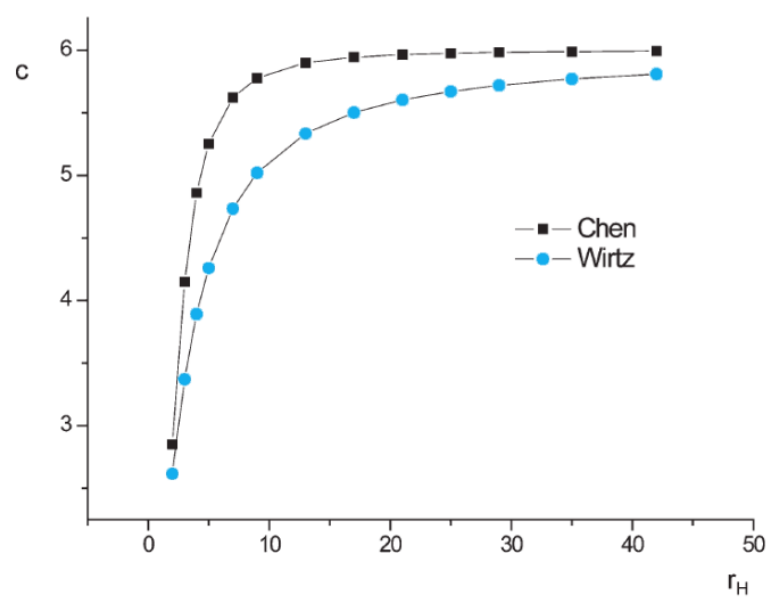

Figure 1-10: Comparison of the approaches of Wirtz ${ }^{[95]}$ and Chen ${ }^{[96]}$ on the correctional factor $c$ correlated with a change in hydrodynamic radius (size of the solute). Adapted from reference [80d]. Copyright (c) 2008 The Royal Society of Chemistry. 
The shape correctional factor $f_{\mathrm{s}}$ can be based on early results of Perrin.${ }^{[97]} \mathrm{He}$ differentiated prolate and oblate ellipsoid molecules depending on the ratio of their minor $(b)$ and major $(a)$ semiaxes (see Figure 1-11).

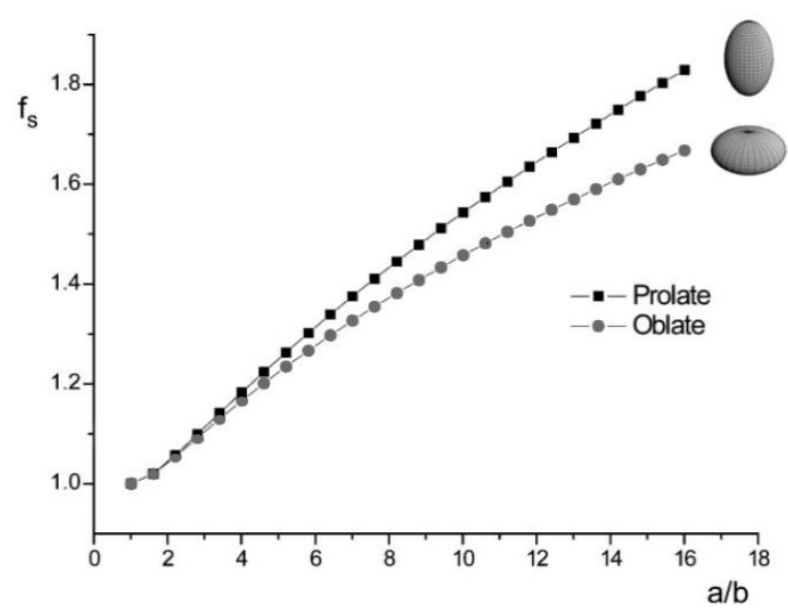

Figure 1-11: Dependence of the shape correctional factor $f_{\mathrm{s}}$ for prolate and oblate ellipsoid molecules. Adapted from reference [80d]. Copyright (c) 2008 The Royal Society of Chemistry.

In 2013, Morris et al. ${ }^{[98]}$ improved the aforementioned Stokes-Einstein based equations. They used the effective solvent density ( $\left.\rho_{\text {eff }}\right)$ instead of solvent radii and were able to drastically increase the average (av.) accuracy (up to about 15\%) of the calculated hydrodynamic radii $\left(r_{\mathrm{H}}\right)$, especially in regard to small molecules.

These calculated molecular sizes in form of $r_{\mathrm{S}}$ or $r_{\mathrm{H}}$ need to be compared to other theoretically or experimentally accessible radii, e.g. van-der-Waals radii $\left(r_{\mathrm{vdW}}\right)$ or radii obtained from X-ray crystallography $\left(r_{\mathrm{X} \text {-ray }}\right)$. While $r_{\mathrm{vdW}}$ are in good agreement with $r_{\mathrm{S}}$ for very spherical molecules, they completely fail for molecules with any inlets or cavities. Since $r_{\mathrm{vdw}}$ are based on the assumption that molecules are made up of perfect spheres, they naturally represent the lower limit of radii. In contrast, radii obtained by X-ray crystallography are larger than $r_{\mathrm{S}}$ and correlate best for non-spherical molecules incorporating inlets. ${ }^{[80 \mathrm{~d}]}$ In light of these facts, it is essential to know what type of molecule and aggregation is to be interpreted and what kind of information is already available for it, so that the right conclusions are drawn from calculated hydrodynamic radii or other information based on these.

\subsubsection{MW Estimation Using Power Law Approaches}

There are several formulae to estimate molecular weights ( $M W \mathrm{~s})$ from the aforementioned radii. However, these incorporate at least the same shortcomings and errors as the radii themselves since they are all based on the Stokes-Einstein equation. Recently, an empirical power law has become the center of attention for the correlation of measured diffusion coefficients to MWs (see equation $(1-6))$. 


$$
D \propto M W^{-\alpha}
$$

In this correlation, $\alpha$ is a parameter that incorporates molecular properties and that is also often referred $^{[99]}$ to as the Flory exponent. That is because Flory ${ }^{[100]}$ established a similar relation for polymers and proteins in the 1950s. A Flory exponent of $\alpha=1$ is achieved for completely linear molecules, while a Flory exponent of $\alpha=1 / 3$ is related to objects or molecules that completely fill up all available space. Therefore, this exponent is a measure for molecular compactness amongst others. While, like in the case of Flory, power laws were and are mostly used for larger molecules (e.g. for polymers ${ }^{[99,101]}$, hollow clusters ${ }^{[102]}$ or biomolecules ${ }^{[103]}$ ), Crutchfield and Harris ${ }^{[104]}$ could show in 2007 that even for small molecules a greatly improved accuracy can be accomplished compared to Stokes-Einstein approaches. Since diffusion measurements themselves and therefore absolute diffusion coefficients are strongly influenced by temperature, viscosity, concentration and instrument specific variables (e.g. gradient strength), there was need for a complimentary technique. Harris et al. ${ }^{[104]}$ chose to relate diffusion coefficients of analytes to those of internal references which they believed are "conveniently present in common NMR solvents", like tetramethylsilane (TMS) or water. The av. deviation of estimated MWs using this method was according to Harris et al. only $11 \%$ (maximum deviations were $35 \%$ ), while the lowest average deviation of the Stokes-Einstein based estimations was 15\% (Morris ${ }^{\text {[98] }}$ approach). The independence of viscosity for such relations (see equation (1-7)) could already be shown earlier. ${ }^{[105]}$

$$
D_{\text {rel, ref }}=\frac{D_{\text {ref }}}{D_{\mathrm{x}}}
$$

Here $D_{\text {ref }}$ is the diffusion coefficient of an internal reference and $D_{\mathrm{x}}$ of an analyte x. Dobson et al. ${ }^{[105 a]}$ termed this ratio relative diffusivity $\left(D_{\text {rel,ref }}\right)$. Harris et al. ${ }^{[104]}$ used this relation to obtain relative diffusivity vs. $M W$ calibration curves in $\mathrm{CDCl}_{3}$ and $\mathrm{D}_{2} \mathrm{O}$. They also showed that those relative diffusivities are widely constant in various solvents (variations of up to $25 \%$ ). However, through their calibration curves they also showed that differentiating solvent environments is vital (see Figure 1-12).

Another approach to estimate MWs from diffusion coefficients was introduced by Williard et al.: ${ }^{[106]}$ They used at least three different internal references (see Figure 1-13) with known $M W$ s to establish internal calibration curves (ICCs). Because these references as well as the analytes were in the same sample, they were exposed to the same temperature, viscosity, etc. which made their diffusion coefficients mutually comparable. Using a logarithmic form of the power law (see equation (1-8)) they were able to estimate $M W$ s of the remaining solutes using a linear fit of a plot of logarithmic diffusion coefficients against logarithmic molecular weights.

$$
\log (D)=\log (K)-\alpha \cdot \log (M W)
$$




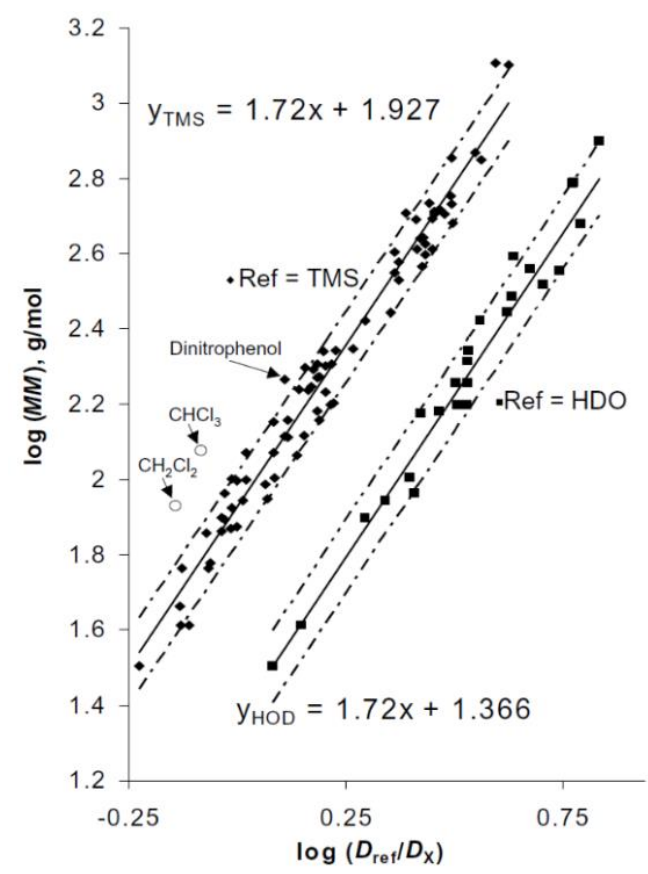

Figure 1-12: Calibration curves of a multitude of references in $\mathrm{CDCl}_{3}$ (diamonds) and $\mathrm{D}_{2} \mathrm{O}$ (squares) using their logarithmic relative diffusivities and molecular weights. Reprinted from reference [104]. Copyright (c) 2007 Elsevier.

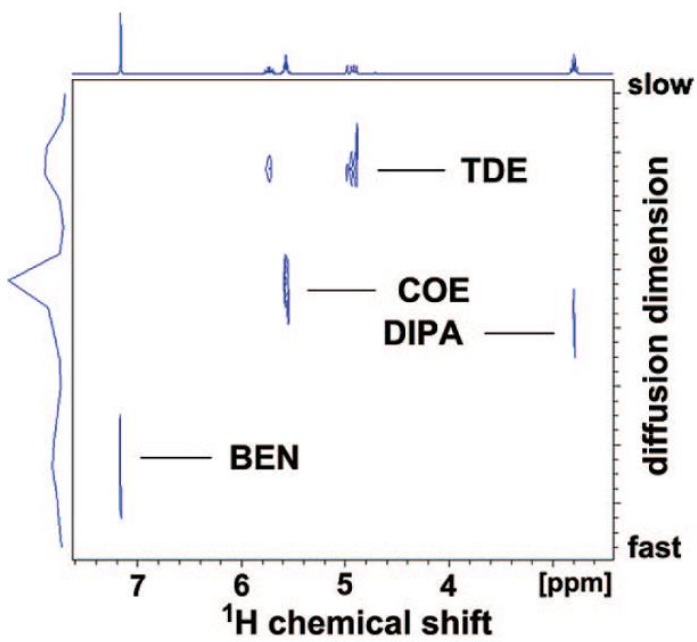

Figure 1-13: ${ }^{1} \mathrm{H}$-DOSY spectrum showcasing several internal references (1-tetracene (TDE); cyclooctene (COE) and benzene (BEN)). These can later be used to form an ICC and estimate the MWs of other solutes (in this case of diisopropylamide (DIPA)). Reprinted from reference [106b]. Copyright (C) 2009 American Chemical Society.

This ICC methodology was used extensively to estimate $M W$ s of a multitude of compounds (e.g. organometallics) and deduce their solution state structures. ${ }^{[107]}$ Moreover, Williard et al. ${ }^{[108]}$ were able to extend the methodology towards heteronuclei. There are still a few setbacks with this technique that are on the one hand related to the needed reference count (a minimum of three) and the limited applicability (an ICC is only viable for a single NMR sample and experiment). Furthermore, this technique is in the same accuracy region as Harris' normalization approach.

Very recently, our group was able to develop a new method on the basis of this ICC technique of Williard. ${ }^{[109]}$ As was already mentioned, Williard's method required a multitude of different 
references present in an NMR sample which produces a number of complications, since the references needed to possess certain properties. They needed to be inert, should not produce signal overlap or interact in any way with other solutes, especially not with the analytes. They should also be well soluble and have a good $M W$ distribution so that the subsequent $M W$ estimation is based on a wide range of $M W$ s. It is difficult to accommodate all these criteria, since especially signal overlap is a prevalent problem. Normalizing diffusion coefficients similar to Harris approach proved to be the easiest way to produce comparability, while only utilizing a single reference. Such normalized diffusion coefficients can be arranged in the same way as in ICCs by simply plotting them logarithmically against known logarithmic $M W$ s (see Figure 1-14, left).
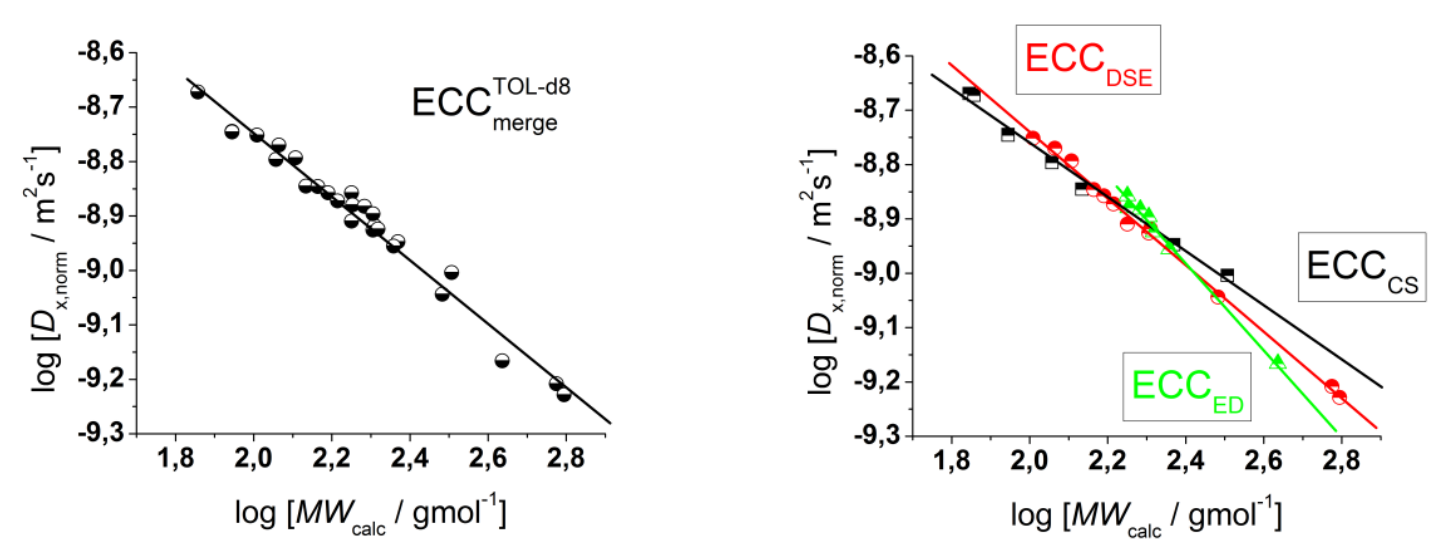

Figure 1-14: External calibration curves (ECCs) of several references in toluene. Molecules with very high or very low $M W$ deviate in some cases more strongly from an averaged ECC (left). This can be improved by using separate ECCs based on molecular shape (right). Reprinted from reference [109a]. Copyright $\odot 2015$ The Royal Society of Chemistry.

Since all diffusion coefficients were normalized, they also did not need to be measured at the same time, but rather in different samples and experiments which made it possible to establish a wider, more extensive $M W$ distribution. The resulting logarithmic plots were named external calibration curves (ECCs).

It was already mentioned for the Stokes-Einstein approaches that deriving molecular sizes or masses from diffusion coefficients is tightly related to their shapes. Therefore, the accuracy of the ECC method was further increased by differentiating between molecular shapes (see Figure 1-14, right). ${ }^{[109 a]}$ This was done by semi-empirically dividing the compounds used for calibration into three categories (the categorization process will be explained in more detail in section 2.1): Compact spheres (CS), dissipated spheres and ellipsoids (DSE) or expanded discs (ED) (see Figure 1-15). After this categorization, MWs could be estimated with the resulting ECCs with a maximum deviation of $9 \%$ in until now two solvents (THF- $d_{8}$ and toluene- $d_{8}$ ) which was deemed an immense improvement compared to averaged ECCs. ${ }^{a}$ Using this method, our group was able to solve the solution structures of lithium diisopropylamide ${ }^{[110]}$ as well as several Hauser bases. ${ }^{[11]}$ Moreover, the methodology has already been adopted by other groups: Mulvey et al. ${ }^{[12]}$

${ }^{a}$ Deviations of the average ECCs were not given in reference ${ }^{[109]}$. 
investigated alkali metal and alkali metal magnesiate amido systems, Wang and Pedersen et al. ${ }^{[13]}$ glycosylation intermediates and O'Hara et al. ${ }^{[14]}$ alkali metal hexamethyldisilazides using ECCs.
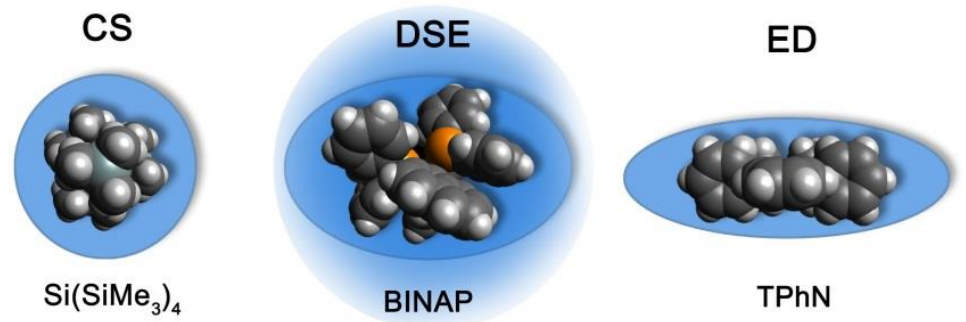

Figure 1-15: Different molecular shapes defined for ECC-DOSY: Compact spheres (CS), dissipated spheres and ellipsoids (DSE) and expanded discs (ED). Reprinted from reference [109a]. Copyright (c) 2015 The Royal Society of Chemistry.

Even though all the herein-presented $M W$ estimations from diffusion data may never be as precise as mass spectrometry, it was proven that they hold great value for the structure determination in solution.

\subsection{Scope of this Thesis}

It is the aim of this thesis to improve and apply the recently developed ECC-DOSY- $M W$ estimation methodology which can aid in a more straightforward solvation of occurring ambiguities related to equilibria in solution via NMR spectroscopy. This is important since aggregates within these equilibria may determine the result of a reaction.

In the first part of this work, the ECC-DOSY- $M W$ estimation methodology itself is reviewed, improved and extended to assure an easy and robust application. In particular, the limited applicability is addressed, since ECCs were only available for THF- $d_{8}$ and toluene- $d_{8}$. Therefore, creating new external calibration curves for other solvent environments is a key element of this work (see Figure 1-16 (left)). Furthermore, most of the research regarding ECCs has been solely empirical; a theoretical calculation of the limits of the ECC- $M W$ estimation as well as a theoretical approach towards the categorization of model compounds according to their shapes is pursued.
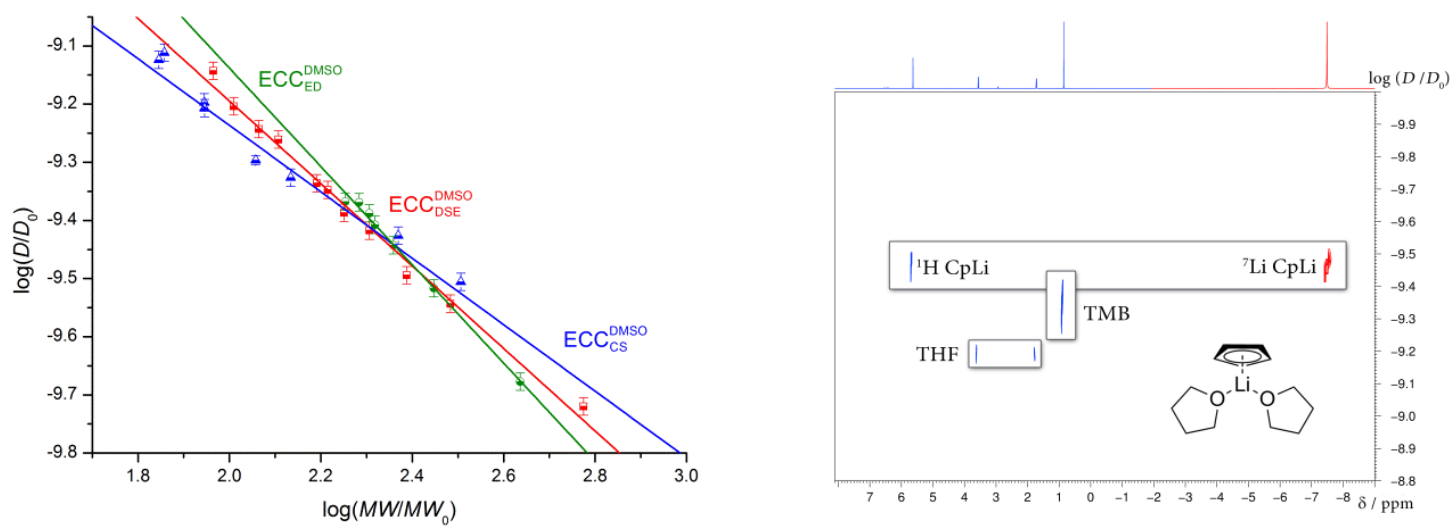

Figure 1-16: Illustrations of the advancement and application of the ECC-DOSY methodology presented in this work. 
The second section features the application of the ECC methodology towards alkali metal cyclopentadienide derivatives in different solvents (see Figure 1-16 (right)). Prior to this work they have been characterized extensively and different aggregational states have been established in the solid state (see section 1.1.2.4). These results can be compared to solution state structures deduced from ECC-DOSY. Moreover, Paquette et al. ${ }^{[66]}$ observed CpLi in THF and stated that they could not "tell whether the monomer-dimer equilibrium [...] is shifted to either side at room temperature". This question is addressed in greater detail and exemplified via ECC-DOSY.

In the third and final part of this work, different ambiguities concerning various organometallic compounds shall be clarified using DOSY: Aside from several potential catalyst and sensor materials, for which simple characterization as well as identifying ion or solvent interactions are envisioned, especially alkyl Grignard systems are in the focus of this section. Here, solution state structures estimated by ECC-DOSY are compared to high-resolution ESI mass spectrometry results from the Koszinowski group, who found trimeric species of alkyl Grignard compounds which extend the classical Schlenk equilibrium. 


\section{RESULTS AND DISCUSSION}

\subsection{Improving the External Calibration Curve (ECC)-DOSY-MW Estimation Methodology}

The external calibration curve (ECC) methodology was developed in our group ${ }^{[109 a]}$ just recently and has already resulted in a multitude of important characterizations of organometallic molecules in solution using DOSY NMR. ${ }^{[10-115]}$ Some of the basics of the methodology have been discussed in section 1.2.3.1, however they will be extended in this chapter, if special considerations are required. Herein, measurements were mostly performed at $25^{\circ} \mathrm{C}$ and low concentrations (15-25 mM), while the methodology was proven to be resilient towards changes in temperature from $-75^{\circ} \mathrm{C}$ to $100^{\circ} \mathrm{C}$ and concentrations of up to $120 \mathrm{mM}$ in $\mathrm{THF}^{\mathrm{a}}$ and toluene. ${ }^{[109 \mathrm{a}]}$ However, there are other issues remaining which will be addressed in this chapter, i.e. the limited applicability in only two solvents, the empirical molecule categorization, the theoretical limitations of the ECCs and the $M W$ prediction for molecules incorporating heavier atoms. Furthermore, the ECC methodology shall be made accessible for a wider scope of application by introducing a user-friendly software application.

\subsubsection{New External Calibration Curves for Various Solvents ${ }^{\mathrm{b}}$}

One of the major tasks for the improvement of the ECC methodology is the establishment of new calibration curves for different deuterated solvents. Up until now ECCs were available for THF- $d_{8}$ and toluene- $d_{8}$ only, however, there are other solvents frequently used in solution state NMR spectroscopy. In this work, the portfolio of ECCs will be extended towards chloroform- $d_{1}\left(\mathrm{CDCl}_{3}\right)$, dichloromethane- $d_{2} \quad\left(\mathrm{CD}_{2} \mathrm{Cl}_{2}\right)$, benzene- $d_{6} \quad\left(\mathrm{C}_{6} \mathrm{D}_{6}\right)$, cyclohexane- $d_{12} \quad\left(\mathrm{C}_{6} \mathrm{D}_{12}\right.$ or $\left.\mathrm{Cycl}\right)$, dimethylsulfoxide- $d_{6}$ (DMSO- $\left.d_{6}\right)$, methanol- $d_{4}\left(\mathrm{CD}_{3} \mathrm{OD}\right)$ and acetonitrile- $d_{3}\left(\mathrm{CD}_{3} \mathrm{CN}\right)$. To establish new ECCs for these solvents, fixed logarithmic diffusion coefficients of specific internal references $\left(\log \left(D_{\text {ref,fix }}\right)\right)$ are needed for the normalization of other diffusion coefficients according to equation (2-1).

$$
\log \left(D_{\mathrm{x}, \mathrm{norm}}\right)=\log \left(D_{\mathrm{x}}\right)+\left(\log \left(D_{\text {ref,fix }}\right)-\log \left(D_{\text {ref }}\right)\right)
$$

These fixed diffusion coefficients $\left(D_{\text {ref,fix }}\right)$ were generated by averaging the results from multiple separate DOSY measurements (at least five). The addition of one of these internal references to an

\footnotetext{
${ }^{a}$ Note that for temperature-dependent measurements, solvent signals should not be used as internal reference. It was shown that at especially lower temperatures deviations of predicted $M W$ s increase. ${ }^{[109 a]}$

${ }^{b}$ Some results of this section have been published separately: S. Bachmann, R. Neufeld, M. Dzemski, D. Stalke, Chem. Eur. J. 2016, 22, 8462-8465. ${ }^{[2]}$
} 
NMR sample together with an analyte $\mathrm{x}$ and application of their measured $\left(D_{\mathrm{x}}\right.$ and $\left.D_{\mathrm{ref}}\right)$ and the fixed diffusion coefficient to equation (2-1) results in normalized diffusion coefficients $\left(D_{x, \text { norm }}\right)$ of the analyte. All normalized diffusion coefficients correlated to the same fixed diffusion coefficient are then mutually comparable and therefore independent of temperature, viscosity and instrumentspecific properties. This is especially useful for the comparison of measurements of different samples, as can be underlined by Figure 2-1.

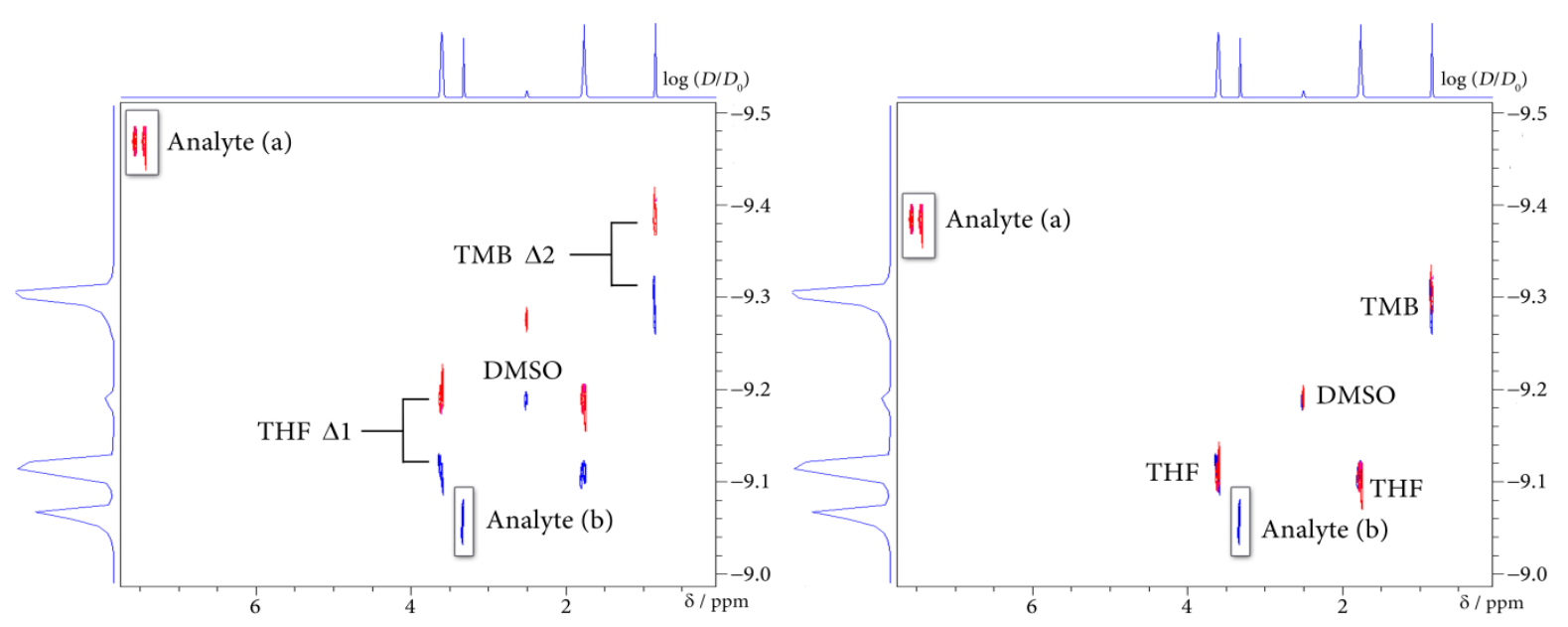

Figure 2-1: Superposition of two ${ }^{1} \mathrm{H}$-DOSY NMR spectra of different samples measured at different instruments and temperatures (red and blue). Before normalization, the individual compounds (analyte (a) and (b)) of these samples are not comparable. Absolute diffusion coefficients of the same compounds (THF, TMB) are different, but differ by the same amount $(\Delta 1=\Delta 2 \neq 0$; left). References can be locked on their fixed diffusion values; all other diffusion coefficients are normalized at the same time. The normalized diffusion coefficients are now mutually comparable. The same compounds (THF, TMB) receive identical values $\left(\Delta 1=\Delta 2=0\right.$; right). $\left(D_{0}=1 \mathrm{~m}^{2} / \mathrm{s}\right)$

For $\mathrm{C}_{6} \mathrm{D}_{12}, \mathrm{CDCl}_{3}, \mathrm{CD}_{2} \mathrm{Cl}_{2}$ and $\mathrm{C}_{6} \mathrm{D}_{6}$ adamantane (Adam) was used as internal reference which produces two signals at about 1.9 and $1.8 \mathrm{ppm}$. Since Adam was barely soluble in DMSO- $d_{6}$, $\mathrm{CD}_{3} \mathrm{OD}$ and $\mathrm{CD}_{3} \mathrm{CN}, 2,2,3,3$-tetramethylbutane $(\mathrm{TMB})$ was used instead. TMB produces a single singlet at about $0.85 \mathrm{ppm}$. Their logarithmic fixed diffusion coefficients are given in Table 2-1.

Table 2-1: $\log \left(D_{\text {ref,fix }}\right)$ of internal references.

\begin{tabular}{lcc}
\hline Solvent & Internal reference & $\log \left(D_{\text {ref,fix }}\right)$ \\
\hline $\mathbf{C}_{6} \mathbf{D}_{6}$ & Adam & -8.8025 \\
$\mathrm{C}_{6} \mathbf{D}_{12}$ & Adam & -9.0204 \\
$\mathrm{CD}_{2} \mathrm{Cl}_{2}$ & Adam & -8.7035 \\
$\mathrm{CDCl}_{3}$ & Adam & -8.8155 \\
$\mathrm{CD}_{3} \mathrm{CN}$ & $\mathrm{TMB}$ & -8.5464 \\
$\mathrm{CD}_{3} \mathrm{OD}$ & $\mathrm{TMB}$ & -8.7737 \\
$\mathrm{DMSO}_{-} \boldsymbol{d}_{6}$ & $\mathrm{TMB}$ & -9.2963 \\
\hline
\end{tabular}

Several compounds were measured together with these internal references for each solvent and their diffusion coefficients were afterwards normalized according to equation (2-1). This way, these model compounds can be used as internal references themselves $\left(\log \left(D_{\mathrm{x}, \mathrm{norm}}\right)=\log \left(D_{\text {ref,fix }}\right)\right)$, as $\operatorname{long}$ as they are available in the respective normalized system. Hence, tables with normalized diffusion 
values of up to 31 model compounds for each solvent are given in the appendix of this work (Table 5-5 (DMSO- $\left.d_{6}\right)$, Table 5-9 $\left(\mathrm{C}_{6} \mathrm{D}_{12}\right)$, Table 5-11 $\left(\mathrm{CDCl}_{3}\right)$, Table 5-13 $\left(\mathrm{CD}_{2} \mathrm{Cl}_{2}\right)$, Table 5-15 $\left(\mathrm{C}_{6} \mathrm{D}_{6}\right)$, Table 5-17 ( $\left.\mathrm{CD}_{3} \mathrm{OD}\right)$ and Table 5-19 $\left.\left(\mathrm{CD}_{3} \mathrm{CN}\right)\right)$. A small selection of these model compounds that can preferably be used as internal references and their respective normalized diffusion coefficients for each solvent are presented in Table 2-2.

Table 2-2: $\log \left(D_{\mathrm{x}, \mathrm{norm}}\right)=\log \left(D_{\text {ref }, \text { fix }}\right)$ for a selection of model compounds that may be used as internal references.

\begin{tabular}{|c|c|c|c|c|c|c|c|}
\hline $\begin{array}{l}\text { Solvent } \\
\text { Reference }\end{array}$ & $\begin{array}{c}\mathrm{C}_{6} \mathrm{D}_{6} \\
\log \left(D_{\mathrm{x}, \text { norm }}\right) \\
\end{array}$ & $\begin{array}{c}\mathrm{C}_{6} \mathrm{D}_{12} \\
\log \left(D_{\mathrm{x}, \text { norm }}\right) \\
\end{array}$ & $\begin{array}{c}\mathrm{CDCl}_{3} \\
\log \left(D_{\mathrm{x}, \text { norm }}\right) \\
\end{array}$ & $\begin{array}{c}\mathrm{CD}_{2} \mathrm{Cl}_{2} \\
\log \left(D_{\mathrm{x}, \text { norm }}\right) \\
\end{array}$ & $\begin{array}{c}\mathrm{CD}_{3} \mathrm{CN} \\
\log \left(D_{\mathrm{x}, \text { norm }}\right) \\
\end{array}$ & $\begin{array}{c}\mathrm{CD}_{3} \mathrm{OD} \\
\log \left(D_{\mathrm{x}, \text { norm }}\right) \\
\end{array}$ & $\begin{array}{c}\text { DMSO- } d_{6} \\
\log \left(D_{x, \text { norm }}\right) \\
\end{array}$ \\
\hline 1-PhN & -8.9085 & -9.0418 & -8.8925 & -8.7860 & -8.6612 & -8.8796 & - \\
\hline DHBP & -8.9793 & -9.1570 & -8.9745 & -8.8623 & -8.7428 & -8.9766 & -9.5168 \\
\hline Adam & -8.8025 & -9.0204 & -8.8155 & -8.7035 & -8.5630 & -8.8025 & -9.3262 \\
\hline Anthracene & -8.8400 & -8.9495 & -8.8142 & -8.6949 & -8.5909 & -8.8080 & - \\
\hline $\mathrm{C}_{6} \mathrm{D}_{6}{ }^{*}$ & -8.6894 & - & - & - & - & - & - \\
\hline $\mathrm{C}_{6} \mathrm{D}_{12}{ }^{*}$ & - & -8.8827 & - & - & - & - & - \\
\hline $\mathrm{CDCl}_{3}{ }^{*}$ & - & - & -8.6580 & - & - & - & - \\
\hline $\mathrm{CD}_{2} \mathrm{Cl}_{2}{ }^{*}$ & - & - & - & -8.4982 & - & - & - \\
\hline $\mathrm{CD}_{3} \mathrm{CN}^{*}$ & - & - & - & - & -8.3784 & - & - \\
\hline $\mathrm{CD}_{3} \mathrm{OD}^{*}$ & - & - & - & - & - & -8.6926 & - \\
\hline Cyclopentane & -8.6276 & -8.8091 & -8.6510 & -8.5514 & -8.4072 & -8.6247 & -9.1238 \\
\hline DPA & -8.8749 & -8.9949 & -8.8589 & -8.7536 & -8.6259 & -8.8541 & -9.3869 \\
\hline DMSO- $d_{6}{ }^{*}$ & - & - & - & - & - & - & -9.1787 \\
\hline Indene & -8.7533 & -8.8679 & -8.7482 & -8.6462 & -8.5183 & -8.7204 & -9.2429 \\
\hline Naphthalene & -8.7650 & -8.8697 & -8.7453 & -8.6439 & -8.5290 & -8.7382 & -9.2609 \\
\hline Pyrene & -8.8698 & -8.9830 & -8.8431 & -8.7386 & -8.6302 & -8.8571 & -9.3876 \\
\hline $\mathrm{Si}\left(\mathrm{SiMe}_{3}\right)_{4}$ & -8.9698 & -9.1839 & -8.9689 & -8.8717 & -8.7317 & -9.0128 & -9.5058 \\
\hline TPhN & -9.1408 & -9.3471 & -9.1166 & -9.0069 & -8.8750 & -9.1068 & -9.6772 \\
\hline TMB & -8.7771 & -8.9783 & -8.7889 & -8.6789 & -8.5464 & -8.7737 & -9.2963 \\
\hline TMS & -8.7144 & -8.8781 & -8.7237 & -8.6252 & -8.4916 & -8.7222 & -9.2037 \\
\hline Toluene & -8.6900 & -8.7966 & -8.6864 & -8.5687 & -8.4630 & -8.6650 & -9.1423 \\
\hline
\end{tabular}

Residual solvent signals are also included in Table 2-2. Still, for these solvents as well as for any other compound that is to be used as internal reference, it must be kept in mind that they should not interact with other solutes or cause signal overlap in the final spectrum, as that can influence the results which leads to the wrong conclusions.

\subsubsection{Merged External Calibration Curves}

After the normalization process, Williard's approach ${ }^{[106 a]}$ (see section 1.2.3.1 for a short summary) of plotting logarithmic diffusion coefficients $\left(\log \left(D_{\mathrm{x}, \mathrm{norm}}\right)\right)$ against logarithmic molecular weights $\left(\log \left(M W_{\text {calc }}\right)\right)$ can be employed to receive a linear dependence. A linear fit (calibration curve) provides the parameters $\log (K)$ (intercept) and $-\alpha$ (slope) which can afterwards be used in formulae (2-2) and (2-3) to estimate $M W s$ ( $M W_{\text {det }}$ ).

$$
\begin{gathered}
\log \left(D_{\mathrm{x}, \text { norm }}\right)=\log (K)-\alpha \cdot \log \left(M W_{\mathrm{det}}\right) \\
\Rightarrow M W_{\mathrm{det}}=10\left(\frac{-\log \left(D_{\mathrm{X}, \text { norm }}\right)+\log (K)}{\alpha}\right)
\end{gathered}
$$


In this work, the normalized diffusion values of model compounds are obtained from many separate measurements with only one internal reference, as mentioned before. Therefore, the corresponding calibration curves are called external calibration curves (ECCs), in contrast to the internal calibration curves (ICCs) of Williard who measured all references and analytes at the same time. This makes a wider range of model compounds accessible (e.g. concerning $M W$ distribution). In this work, small molecules with $M W \mathrm{~s}$ ranging from 70 to $600 \mathrm{~g} / \mathrm{mol}$ were used. MWs of analytes with diffusion coefficients corresponding to $M W$ s inside the given appropriate range can safely be predicted. ${ }^{a}$ If these ECCs incorporate all measured model compounds without any discrimination, they are called "merged" calibration curves (two examples are shown in Figure 2-2; the complete set of merged ECCs for all solvents is given in the appendix: Figure 5-1 (DMSO); Figure 5-2 $\left(\mathrm{C}_{6} \mathrm{D}_{12}\right)$; Figure 5-3 $\left(\mathrm{CDCl}_{3}\right)$; Figure 5-4 $\left(\mathrm{CD}_{2} \mathrm{Cl}_{2}\right)$; Figure 5-5 $\left(\mathrm{C}_{6} \mathrm{D}_{6}\right)$; Figure 5-6 $\left(\mathrm{CD}_{3} \mathrm{OD}\right)$ and Figure 5-7 $\left.\left(\mathrm{CD}_{3} \mathrm{CN}\right)\right)$.
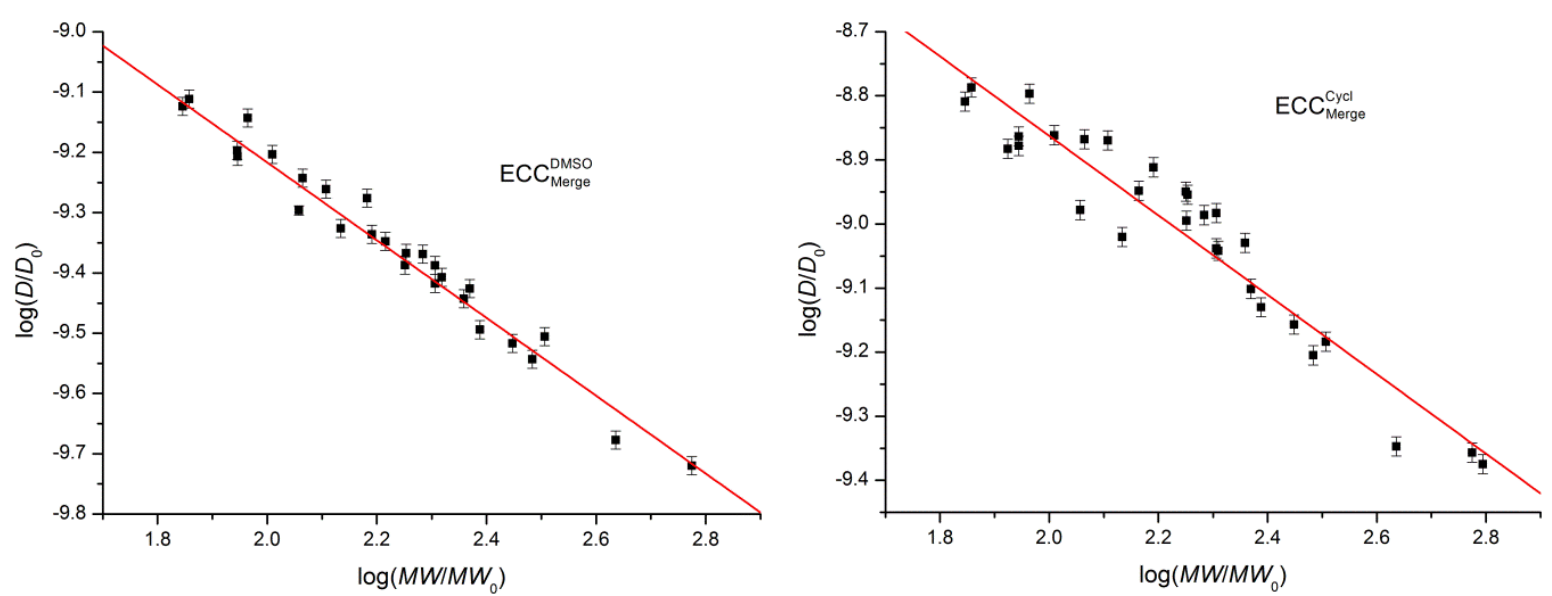

Figure 2-2: Merged ECCs for DMSO- $d_{6}$ and cyclohexane- $d_{12}(\mathrm{Cycl})$. For some solvents (e.g. DMSO) the deviations are rather small, while in other cases (e.g. Cycl) some molecules deviate heavily. $\left(D_{0}=1 \mathrm{~m}^{2} / \mathrm{s} ; M W_{0}=1 \mathrm{~g} / \mathrm{mol}\right)$

The ECC parameters $(\log (K),-\alpha$ and their errors) of these merged ECCs are given in Table 2-3 for all solvents. In the original publication, ${ }^{[109 a]}$ the approach towards merged ECCs was different (see comments in section 2.1.1.2) which required the repetition with normalized diffusion coefficients given in literature. ${ }^{[109 a]}$ The resulting ECC parameters for THF- $d_{8}$ and toluene- $d_{8}$ have also been listed in Table 2-3. At this point it should be noted that three significant digits were used for the ECC parameters as more would overstate the accuracy of the method. This is why some of the estimated $M W$ s of organometallic compounds that are discussed in the following chapters are differing slightly from literature. ${ }^{[2-3,109]}$ To estimate the suitability of ECCs for $M W$ predictions, $M W$ s were back-calculated for all model compounds $\left(M W_{\text {det }}\right)$ and compared with their true molecular weights $\left(M W_{\text {calc }}\right)$. Av. and maximum (max.) deviations (dev.; $M W_{\text {dif }}$ ) of these estimated $M W$ s are also listed in Table 2-3. These deviations were calculated with equation (2-4). $[2,109 \mathrm{a}], \mathrm{b}$

\footnotetext{
a The corresponding diffusion coefficient range is different for each solvent.

b The formula $M W_{\text {dif }}=\left(1-\frac{M W_{\text {det }}}{M W_{\text {calc }}}\right)$ used in literature ${ }^{[2,109]}$ leads to different deviations, if an estimated $M W_{\text {det }}$ is lighter or heavier by the same amount than $M W_{\text {calc }}$, since $M W_{\text {dif }}$ is calculated relative to 1.
} 


$$
M W_{\mathrm{dif}}=\left(\frac{M W_{\mathrm{calc}}-M W_{\mathrm{det}}}{M W_{\mathrm{det}}}\right) \cdot 100 \%
$$

Table 2-3: ECC parameters and their errors, av. and max. deviations of back-calculated $M W s$ and cor. $\mathrm{R}^{2}$ values of merged calibration curves for all solvents available for the ECC method.

\begin{tabular}{|c|c|c|c|c|c|c|c|}
\hline Solvent & $\log (K)$ & $\Delta \log (K)$ & $-\alpha$ & $\Delta \alpha$ & av. dev. & max. dev. & cor. $\mathbf{R}^{2}$ \\
\hline DMSO- $d_{6}$ & -7.93 & 0.0535 & -0.644 & 0.0241 & $\pm 7 \%$ & $\pm 20 \%$ & 0.96 \\
\hline $\mathrm{C}_{6} \mathrm{D}_{12}$ & -7.62 & 0.0831 & -0.620 & 0.0369 & $\pm 15 \%$ & $\pm 29 \%$ & 0.91 \\
\hline $\mathrm{CDCl}_{3}$ & -7.67 & 0.0537 & -0.532 & 0.0239 & $\pm 11 \%$ & $\pm 26 \%$ & 0.94 \\
\hline $\mathrm{CD}_{2} \mathrm{Cl}_{2}$ & -7.55 & 0.0487 & -0.535 & 0.0215 & $\pm 10 \%$ & $\pm 23 \%$ & 0.96 \\
\hline $\mathrm{C}_{6} \mathrm{D}_{6}$ & -7.58 & 0.0380 & -0.572 & 0.0172 & $\pm 8 \%$ & $\pm 20 \%$ & 0.97 \\
\hline $\mathrm{CD}_{3} \mathrm{OD}$ & -7.51 & 0.0555 & -0.600 & 0.0247 & $\pm 9 \%$ & $\pm 22 \%$ & 0.96 \\
\hline $\mathrm{CD}_{3} \mathrm{CN}$ & -7.38 & 0.0456 & -0.553 & 0.0204 & $\pm 8 \%$ & $\pm 19 \%$ & 0.96 \\
\hline THF- $d_{8}$ & -7.60 & 0.0407 & -0.553 & 0.0180 & $\pm 7 \%$ & $\pm 18 \%$ & 0.97 \\
\hline Toluene- $d_{8}$ & -7.59 & 0.0389 & -0.579 & 0.0172 & $\pm 7 \%$ & $\pm 18 \%$ & 0.98 \\
\hline
\end{tabular}

Merged ECCs produce av. deviations of 7-15\% and max. deviations of $18-29 \%$. This is impressive, since all model compounds were considered and no structural differentiation has been done $a$ priori. ${ }^{\text {a }}$ This accuracy enables the verification or falsification of a wide range of aggregates, e.g. a monomeric aggregate can easily be distinguished from dimeric aggregates (this is usually the case, since massive co-aggregation of solvent molecules (solvation) would be necessary to close the $M W$ gap). Additionally, correlated (cor.) $\mathrm{R}^{2}$ values which indicate how good the variation of the points is explained by the fit are given for all merged ECCs. The closer cor. $\mathrm{R}^{2}$ values are to 1 , the better the fit. The cor. $\mathrm{R}^{2}$ values fit in most cases the tendencies of deviations of back-calculated $M W$ s for the different ECCs.

The normalization process for the preparation of the ECCs is similar to the method used by Harris et al. ${ }^{[104]}$ (a short summary of their method can be found in section 1.2.3.1) who employed relative diffusivities $\left(D_{\text {ref,rel }}\right)$. For the ECC methodology, the relative diffusivities are referenced to fixed diffusion coefficients ( $D_{\text {reffixi }}$ ) of internal references as shown in equation (2-5).

$$
\Rightarrow D_{\mathrm{x}, \text { norm }}=\frac{D_{\mathrm{x}}}{D_{\text {ref }}} \cdot D_{\text {ref,fix }}=\frac{1}{D_{\text {ref,rel }}} \cdot D_{\text {ref,fix }}
$$

Harris et al. ${ }^{[104]}$ reported similar relative error limits (11\% av. dev. and 35\% max. dev.; $M W$ distribution of 2 to $1280 \mathrm{~g} / \mathrm{mol}$ ) compared to the merged ECCs which indicates that this normalization is conclusive and transferable. Unfortunately, Harris et al. did not explicitly list their used references or individual diffusion coefficients which hampers the direct comparison between their calibration curve in $\mathrm{CDCl}_{3}$ and the merged ECC of this work. However, Harris et al. ${ }^{[104]}$ plotted $\log \left(D_{\text {ref,rel }}\right)$ vs. $\log \left(M W_{\text {calc }}\right)$ which was fitted linearly. This could be repeated with diffusion coefficients measured herein for $\mathrm{CDCl}_{3}$ (see Figure 2-3).

\footnotetext{
${ }^{a}$ Model compounds with elevated molar van-der-Waals density were excluded $\left(M D_{\mathrm{W}}\right.$; will be explained in section 2.1.4). Note that $\mathrm{Si}(\mathrm{OMe})_{4}$ was removed for this reason and therefore resulting ECCs and conclusions thereof may differ compared to reference [2] (for further information see at the end of section 2.1.1.2).
} 


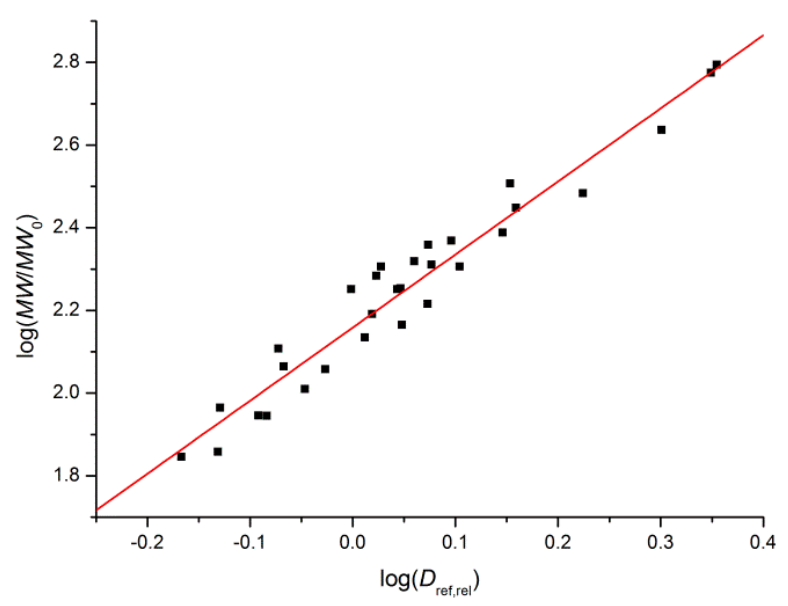

Figure 2-3: Plot of $\log \left(D_{\text {ref,rel }}\right)$ vs. $\log \left(M W_{\text {calc }}\right)$ of measurements in $\mathrm{CDCl}_{3}$. The cor. $\mathrm{R}^{2}(0.94)$ value remains identical in comparison to the conventional plot. $\left(M W_{0}=1 \mathrm{~g} / \mathrm{mol}\right)$

The linear fit parameters of the plot with the data of this work (slope: 1.77; intercept: 2.16) differ slightly compared to those reported by Harris et al.$^{[104]}$ (slope: 1.72 ; intercept: 1.93 ). This is probably due to the different pool of substances utilized by Harris et al. compared to this work. Since the compounds were not given by Harris et al., ${ }^{[104]}$ this could not be investigated.

The usefulness of the normalization process and advantage of the introduction of a standardized system can be further illustrated by the following example. Wang and Pedersen et al. ${ }^{[113]}$ investigated glycosylation intermediates incorporating fluorinated counter ions that are soluble in dichloromethane $\left(\mathrm{CD}_{2} \mathrm{Cl}_{2}\right)$. These are a result of the use of fluorinated catalysts, e.g. $\left[\mathrm{BF}_{3} \cdot \mathrm{Et}_{2} \mathrm{O}\right]$ or [TMSOTf]. They decided to prepare their own ECCs, to account for specific features of these compounds. One model compound Wang and Pedersen et al. used was diethylether $\left(\mathrm{Et}_{2} \mathrm{O}\right)$. To enable cross-referencing, $\mathrm{Et}_{2} \mathrm{O}\left(\log \left(D_{\mathrm{x}, \mathrm{norm}}\right)=-8.5724\right)$ was also measured for this work and normalized according to equation (2-1) in $\mathrm{CD}_{2} \mathrm{Cl}_{2}$. This way, all normalized diffusion coefficients of Wang and Pedersen et al. (see Supporting Information of reference $\left.{ }^{[13]}\right)$ could be converted to the herein used internal standard by repeatedly applying formula (2-1) (A complete list of all diffusion coefficients is given in the appendix: Table 5-25). Figure 2-4 depicts the resulting ECC of Wang and Pedersen et al. converted into the herein used system (red) together with the merged ECC in $\mathrm{CD}_{2} \mathrm{Cl}_{2}$ (black) for comparison. It is immediately obvious that these two shown calibration curves cannot simply be combined. Their slopes are substantially different: $-\alpha\left(\mathrm{ECC}_{\text {merge }}\right)=-0.535$; $-\alpha\left(\mathrm{ECC}_{\text {Wang/Pedersen }}\right)=-0.220$.

This could be due to the used references of Wang and Pedersen et al., however, after careful evaluation this assumption can be dismissed: While $\mathrm{Et}_{2} \mathrm{O}$ (marked in Figure 2-4) would be in a reasonable region for the herein presented merged ECC, the other references that were meant to resemble their investigated aggregates exhibit irregularities. These molecules display mainly an increasingly elevated molar van-der-Waals density ( $M D_{\mathrm{W}}$; A-C shown in Scheme 2-1) which rises continuously along their calibration curve (for 5 of the 6 molecules; see Table 5-25) and effectively 


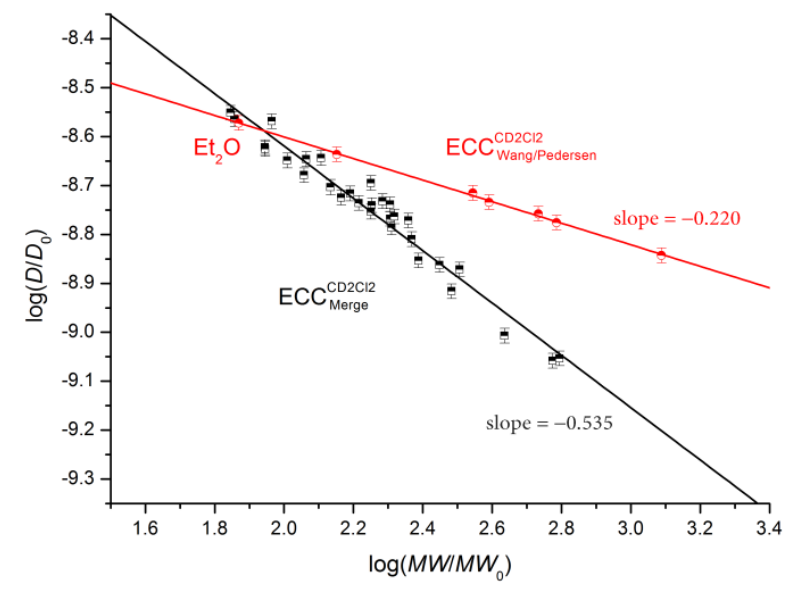

Figure 2-4: Merged ECC for $\mathrm{CD}_{2} \mathrm{Cl}_{2}$ (black) and ECC of Wang and Pedersen et al. ${ }^{[113]}$ (red) converted into the herein used normalized system. Wang and Pedersen et al. used references that produce irregularities, i.e. due to increasing molar vander-Waals-densities or sterically demanding functional groups. $\left(D_{0}=1 \mathrm{~m}^{2} / \mathrm{s} ; M W_{0}=1 \mathrm{~g} / \mathrm{mol}\right)$
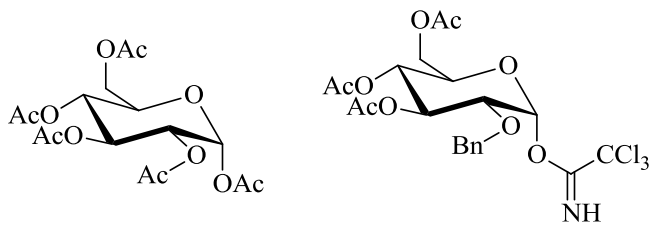

B

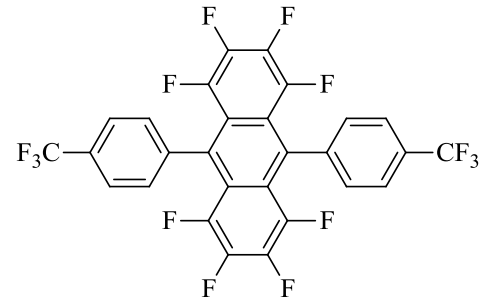

C

$$
M W=390 \mathrm{~g} \mathrm{~mol}^{-1} \quad M W=541 \mathrm{~g} \mathrm{~mol}^{-1} \quad M W=610 \mathrm{~g} \mathrm{~mol}^{-1}
$$

increasing molar van-der-Waals density

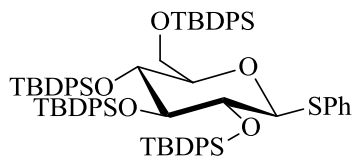

D

$M W=1226 \mathrm{~g} \mathrm{~mol}^{-1}$
TBDPS $=$

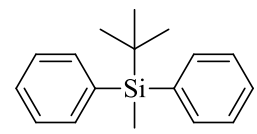

Sterically demanding groups induce cavities or inlets

Scheme 2-1: Selection of utilized references of Wang and Pedersen et al. ${ }^{[113]}$ References A-C show increasing levels of vander-Waals densities, whereas the substituents of reference $\mathbf{D}$ induce substantial inlets and cavities.

induces underestimation of $M W \mathrm{~s}$ which would explain their raised diffusion coefficients $\left(M D_{\mathrm{W}}\right.$ will be discussed in more detail in section 2.1.4). However, their last utilized reference (D shown in Scheme 2-1) has no elevated van-der-Waals density and even possesses enormous cavities and inlets, induced by sterically demanding substituents which would more likely have an opposite effect and lead to an overestimation of $M W$ s. Even without this reference, their slope $\left(-\alpha\left(\right.\right.$ ECC $\left.\left._{\text {Wang/Pedersen }}\right)=-0.220\right)$ does not fit the assumption for a Flory-coefficient $(0.33 \leq \alpha \leq 1)$. Hence, it is probable that there has been an error in their measurement, e.g. due to convection. ${ }^{a}$ While it is unfortunate that these ECCs cannot be combined, it could be made apparent that the normalization process can help detect irregularities.

\footnotetext{
${ }^{a}$ Wang and Pedersen et al. ${ }^{[113]}$ utilized the pulse sequence ledbpgp $2 \mathrm{~s}$ and measured at $-55^{\circ} \mathrm{C}$.
} 


\subsubsection{Shape-Optimized External Calibration Curves}

The shape dependency of $D$ is also the main reason for the elevated max. deviations of merged ECCs. These result mostly from individual model compounds with distinct molecular shapes. Alas, theoretical calculations of diffusion coefficients are currently not precise enough to complement experimental findings (at least for small molecules). ${ }^{[116]}$ Advanced theoretical means achieved by rising computational power may in the future make shape-related discrepancies predictable.

Such geometry-related effects on diffusion experiments were already acknowledged with the correctional factor $\left(f_{S}\right)$ of Perrin ${ }^{[97]}$ for Stokes-Einstein approaches (see section 1.2.3.1), however neither by Harris nor by Williard for their power-law based methods. Therefore, to further improve the accuracy of ECCs, the pool of model compounds was semi-empirically divided into one of the following categories of molecular shapes: Compact spheres (CS), dissipated spheres and ellipsoids (DSE) and expanded discs (ED). ${ }^{[109 a], ~ a ~ C o m p a c t ~ s p h e r i c a l ~ m o l e c u l e s ~ a r e ~ h i g h l y ~ s y m m e t r i c ~(e . g . ~} \mathrm{T}_{\mathrm{d}}$, $\mathrm{O}_{\mathrm{h}}$ ) and densely packed (e.g. tetramethylsilane (TMS) or Adam). However, most model compounds are not ideally spherical, but rather ellipsoidal (e.g. tetramethoxypropane or diphenylsulfoxide (DPS)) or incorporate small cavities/inlets or dative bonds. These are grouped in the DSE category. Moreover, small aromatic molecules like toluene are included, since expanded discs do not seem to behave significantly different before $M W \mathrm{~s}>170 \mathrm{~g} / \mathrm{mol}$. Above $170 \mathrm{~g} / \mathrm{mol}$, larger expanded discs may also be differentiated (e.g. anthracene or pyrene). A complete list of all model compounds and their individual categorization is given in the appendix (Table 5-1). ${ }^{\mathrm{b}}$

Shape-optimized ECCs (using the given procedure) were then created after this geometrical categorization (see Figure 2-5).
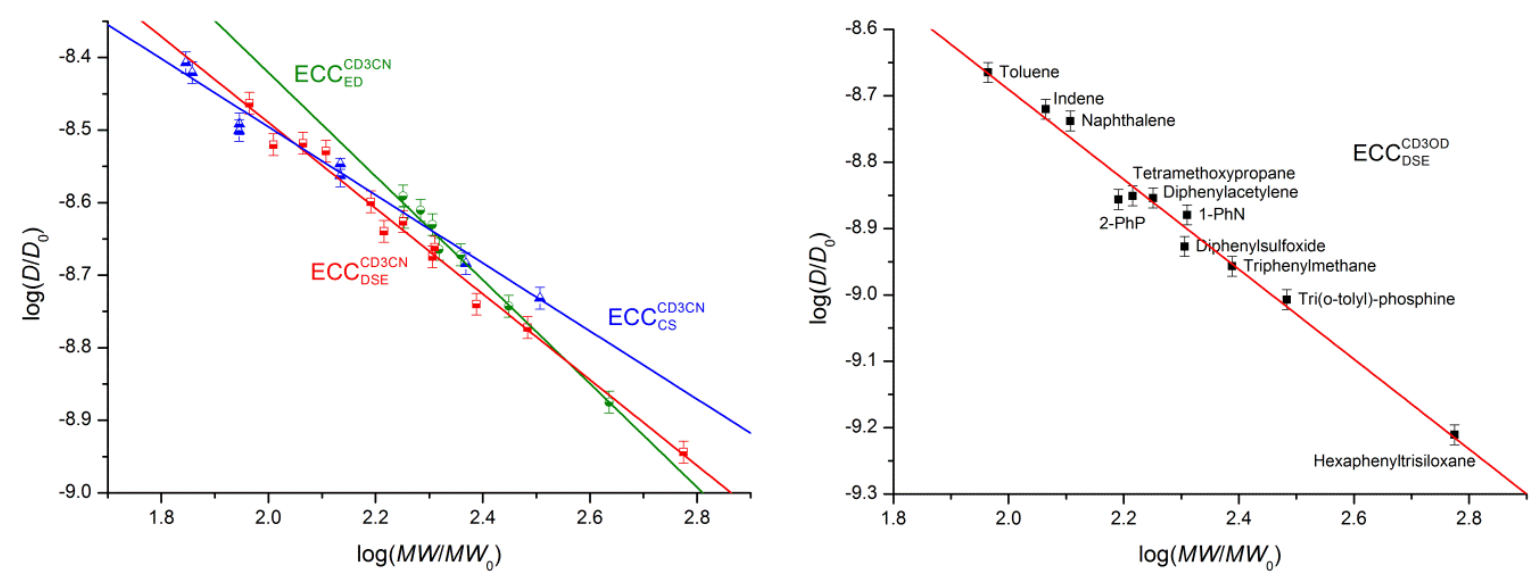

Figure 2-5: Shape-optimized ECCs: Semi-empirical differentiation of molecular shapes leads to three distinct categories: Compact spheres (CS), dissipated spheres and ellipsoids (DSE) and expanded discs (ED). Such categorization is shown for $\mathrm{CD}_{3} \mathrm{CN}$ (left). The individual shape-optimized ECCs show increased accuracy compared to raw merged ECCs, e.g. shape-optimized ECC for DSE molecules in $\mathrm{CD}_{3} \mathrm{OD}$ (right). $\left(D_{0}=1 \mathrm{~m}^{2} / \mathrm{s} ; M W_{0}=1 \mathrm{~g} / \mathrm{mol}\right.$ )

\footnotetext{
a A fourth molecular shape will be introduced and discussed in section 2.3.1.

b There are some borderline molecules, but there are also clear systematic trends.
} 
The complete set of shape-optimized ECCs for all solvents is given in the appendix (Figure 5-1 (DMSO); Figure 5-2 $\left(\mathrm{C}_{6} \mathrm{D}_{12}\right)$; Figure 5-3 $\left(\mathrm{CDCl}_{3}\right)$; Figure 5-4 $\left(\mathrm{CD}_{2} \mathrm{Cl}_{2}\right)$; Figure 5-5 $\left(\mathrm{C}_{6} \mathrm{D}_{6}\right)$; Figure 5-6 $\left(\mathrm{CD}_{3} \mathrm{OD}\right)$ and Figure 5-7 $\left.\left(\mathrm{CD}_{3} \mathrm{CN}\right)\right)$. For most organometallic molecules, the calibration curves for dissipated spheres and ellipsoids (DSE) have been proven to be the best choice for $M W$ estimation. ${ }^{[109-112]}$ Further calibration curves (CS, ED) are suited to discuss molecules with well-defined shapes.

This categorization led to an immense increase in accuracy of back-calculated $M W s$ of the model compounds. However, for shape-optimized ECCs to be applied, the molecular shape of an analyte needs to be known a priori. This is usually a delicate situation and in some cases, where it is not possible to conclude the "right" molecular shape, universal merged ECCs should instead be utilized. In the original work ${ }^{[109 a]}$ such merged ECCs were obtained by averaging the ECC parameters of the three shape-optimized ECCs to equally weigh the shapes. However, it is most probably not suitable, since the shape-optimized ECCs may be biased, e.g. through the categorization of borderline molecules (other approaches towards categorization are discussed in section 2.1.2). Therefore, merged ECCs reported in this work were simply obtained by utilizing all model compounds and weighing them evenly, as explained before. However, if any evidence towards the constitution of an aggregate in solution exists or a reasonable guess can be made, shape-optimized ECCs are still useful.

For $\mathrm{C}_{6} \mathrm{D}_{6}, \mathrm{CDCl}_{3}, \mathrm{CD}_{2} \mathrm{Cl}_{2}, \mathrm{CD}_{3} \mathrm{CN}$ and $\mathrm{CD}_{3} \mathrm{OD}$ calibration curves for the three molecular shapes (ED, CS, and DSE) could be assembled. Their ECC parameters as well as av. and max. deviations and cor. $\mathrm{R}^{2}$ values are given in Table 2-4. Furthermore, av. and max. deviations recalculated via equation (2-4) and errors of the ECC parameters are listed for THF- $d_{8}$ and toluene- $d_{8}$, as these were not listed in the original publication. ${ }^{[109 a]}$ Additionally, the same information is given in Table 2-4 for the ED and DSE calibration curves of DMSO- $d_{6}$ and cyclohexane- $d_{12}$ (problems with the CS calibration curves are discussed later).

The av. deviations of these ECCs vary between 1 and 10\%, while max. deviations stray substantially more ( 3 to $22 \%$ ). A realistic av. deviation of about $5 \%$ can be proposed for these ECCs; anything better should be viewed skeptically. Still, these error limits mark a substantial improvement (around 5 to 15\%) compared to the merged ECCs. The very pronounced max. deviations are still caused by some specific borderline molecules in many of the ECCs: Naphthalene and toluene diffuse a little faster than anticipated for the DSE shape which could be due to their planarity, like for most ED molecules. However, they are essentially too small to be considered for the ED shape, as they would produce even more elevated deviations for the ED ECCs. Also, some oxygencontaining molecules like diisopropylether and tetramethoxypropane tend to diffuse slower than expected; maybe some intermolecular interactions (e.g. with the solvent) cause this behavior. This however, could not be confirmed, yet. 
Table 2-4: ECC parameters and their errors, av. and max. deviations of back-calculated $M W s$ and cor. $\mathrm{R}^{2}$ values of shape-optimized calibration curves (CS, DSE and ED) for $\mathrm{CDCl}_{3}, \mathrm{CD}_{2} \mathrm{Cl}_{2}, \mathrm{C}_{6} \mathrm{D}_{6}, \mathrm{CD}_{3} \mathrm{OD}, \mathrm{CD}_{3} \mathrm{CN}$, THF- $d_{8}$ and toluene- $d_{8}$. The same information is given for shape-optimized calibration curves (DSE and ED) for DMSO- $d_{6}$ and $\mathrm{C}_{6} \mathrm{D}_{12}$.

\begin{tabular}{|c|c|c|c|c|c|c|c|}
\hline & $\log (K)$ & $\Delta \log (K)$ & $-\alpha$ & $\Delta \alpha$ & av. dev. & max. dev. & cor. $\mathrm{R}^{2}$ \\
\hline \multicolumn{8}{|c|}{ Solvent: $\mathrm{CDCl}_{3}$} \\
\hline CS & -7.84 & 0.0487 & -0.457 & 0.0231 & $\pm 6 \%$ & $\pm 18 \%$ & 0.98 \\
\hline DSE & -7.59 & 0.0694 & -0.572 & 0.0302 & $\pm 10 \%$ & $\pm 22 \%$ & 0.97 \\
\hline ED & -7.13 & 0.133 & -0.753 & 0.0565 & $\pm 4 \%$ & $\pm 10 \%$ & 0.96 \\
\hline \multicolumn{8}{|c|}{ Solvent: $\mathrm{CD}_{2} \mathrm{Cl}_{2}$} \\
\hline CS & -7.70 & 0.0354 & -0.469 & 0.0169 & $\pm 4 \%$ & $\pm 7 \%$ & 0.99 \\
\hline DSE & -7.46 & 0.0484 & -0.577 & 0.0210 & $\pm 5 \%$ & $\pm 14 \%$ & 0.98 \\
\hline ED & -6.98 & 0.113 & -0.767 & 0.0480 & $\pm 3 \%$ & $\pm 9 \%$ & 0.97 \\
\hline \multicolumn{8}{|c|}{ Solvent: $\mathrm{C}_{6} \mathrm{D}_{6}$} \\
\hline CS & -7.75 & 0.0545 & -0.494 & 0.0260 & $\pm 7 \%$ & $\pm 17 \%$ & 0.98 \\
\hline DSE & -7.47 & 0.0284 & -0.622 & 0.0124 & $\pm 3 \%$ & $\pm 8 \%$ & 0.99 \\
\hline ED & -7.06 & 0.0861 & -0.787 & 0.0365 & $\pm 3 \%$ & $\pm 6 \%$ & 0.99 \\
\hline \multicolumn{8}{|c|}{ Solvent: $\mathrm{CD}_{3} \mathrm{OD}$} \\
\hline $\mathrm{CS}$ & -7.64 & 0.0809 & -0.539 & 0.0383 & $\pm 5 \%$ & $\pm 13 \%$ & 0.96 \\
\hline DSE & -7.34 & 0.0677 & -0.677 & 0.0296 & $\pm 5 \%$ & $\pm 11 \%$ & 0.98 \\
\hline ED & -7.08 & 0.0530 & -0.773 & 0.0223 & $\pm 2 \%$ & $\pm 3 \%$ & 1.00 \\
\hline \multicolumn{8}{|c|}{ Solvent: $\mathrm{CD}_{3} \mathrm{CN}$} \\
\hline CS & -7.55 & 0.0677 & -0.469 & 0.0320 & $\pm 9 \%$ & $\pm 17 \%$ & 0.97 \\
\hline DSE & -7.31 & 0.0491 & -0.591 & 0.0217 & $\pm 4 \%$ & $\pm 13 \%$ & 0.99 \\
\hline ED & -6.99 & 0.0871 & -0.714 & 0.0369 & $\pm 3 \%$ & $\pm 7 \%$ & 0.98 \\
\hline \multicolumn{8}{|c|}{ Solvent: THF- $d_{8}$} \\
\hline CS & -7.74 & 0.0397 & -0.494 & 0.0187 & $\pm 2 \%$ & $\pm 4 \%$ & 0.99 \\
\hline DSE & -7.54 & 0.0285 & -0.582 & 0.0124 & $\pm 4 \%$ & $\pm 8 \%$ & 0.99 \\
\hline ED & -7.12 & 0.0449 & -0.752 & 0.0191 & $\pm 1 \%$ & $\pm 3 \%$ & 1.00 \\
\hline \multicolumn{8}{|c|}{ Solvent: Toluene- $d_{8}$} \\
\hline CS & -7.76 & 0.0469 & -0.502 & 0.0224 & $\pm 6 \%$ & $\pm 10 \%$ & 0.99 \\
\hline DSE & -7.51 & 0.0246 & -0.613 & 0.0106 & $\pm 3 \%$ & $\pm 7 \%$ & 1.00 \\
\hline ED & -7.10 & 0.0717 & -0.784 & 0.0306 & $\pm 2 \%$ & $\pm 4 \%$ & 0.99 \\
\hline \multicolumn{8}{|c|}{ Solvent: DMSO- $d_{6}$} \\
\hline DSE & -7.78 & 0.0487 & -0.709 & 0.0215 & $\pm 3 \%$ & $\pm 10 \%$ & 0.99 \\
\hline ED & -7.45 & 0.0660 & -0.846 & 0.0278 & $\pm 2 \%$ & $\pm 4 \%$ & 0.99 \\
\hline \multicolumn{8}{|c|}{ Solvent: $\mathrm{C}_{6} \mathrm{D}_{12}$} \\
\hline DSE & -7.41 & 0.0643 & -0.707 & 0.0279 & $\pm 5 \%$ & $\pm 17 \%$ & 0.98 \\
\hline $\mathrm{ED}$ & -6.59 & 0.109 & -1.04 & 0.0461 & $\pm 1 \%$ & $\pm 5 \%$ & 0.99 \\
\hline
\end{tabular}

It was already explained that the slope $(-\alpha)$ is a measure of molecular compactness, which is also reflected in the shape-optimized ECCs. The ECCs for CS compounds have comparably the lowest $\alpha$ values, while they increase over DSE to ED as the molecules get more two-dimensional.

The parameters for shape-optimized calibration curves of CS compounds of DMSO- $d_{6}$ and cyclohexane- $d_{12}$ are deliberately left out, since these ECCs displayed atypical deviations. Linear fitting of the CS-type references resulted in a significantly reduced quality of the fit (cor. $\mathrm{R}^{2}=0.88$ 
for $\mathrm{C}_{6} \mathrm{D}_{12}$ and cor. $\mathrm{R}^{2}=0.92$ for DMSO- $\left.d_{6}\right)$. To exclude aggregation phenomena, two CS references, $\mathrm{Si}\left(\mathrm{SiMe}_{3}\right)_{4}$ and TMS, were measured at different concentrations which resulted in almost identical

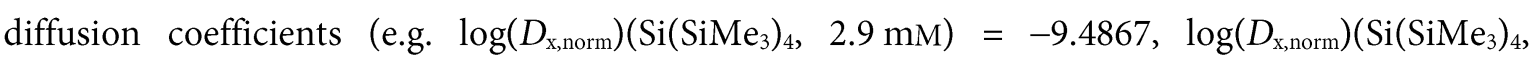

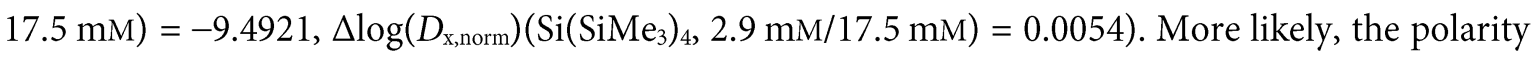
of the solvents causes these deviations in diffusion (note that cyclohexane has no dipole moment, whereas DMSO adopts $\left.3.96 \mathrm{D}^{[117]}\right)$. Still, grouping polar (e.g. THF $1.75 \mathrm{D}^{[117]}, \mathrm{N}\left(\mathrm{SiMe}_{3}\right)_{3} 0.51 \mathrm{D}^{[118]}$ ) and nonpolar (e.g. TMB, TMS) compact spheres together did not result in a classification, in which all references fitted the corresponding ECCs. Furthermore, the slope of these ECCs should be inversed for DMSO and cyclohexane, if polarity was the only responsible property. Measurements have also been performed in dry DMSO to exclude interactions with water. This also did not alter the findings significantly.

In a separate publication, ${ }^{[2]}$ we divided CS compounds into purely hydrocarbon (PCS; e.g. TMB, Adam) and non-hydrocarbon (NCS; e.g. MTBE, $\left.\mathrm{Si}\left(\mathrm{SiMe}_{3}\right)_{4}\right)$ compounds which resulted in a clear increase in accuracy (see reference [2] for deviations, normalized diffusion coefficients and plots). However, different effects of hydrocarbon and non-hydrocarbon compounds on diffusion are questionable and this problem was therefore investigated in more detail since publication: Both solvents in question display an increased viscosity (DMSO: $2.24 \mathrm{mPa} \cdot \mathrm{s}$; cyclohexane: $1.0 \mathrm{mPa} \cdot \mathrm{s}$ $\left(\right.$ at $20^{\circ} \mathrm{C}$ ) ${ }^{[119]}$ compared to other solvents (e.g. chloroform: $0.57 \mathrm{mPa} \cdot \mathrm{s}$; methanol: $0.59 \mathrm{mPa} \cdot \mathrm{s}$ (at $\left.20^{\circ} \mathrm{C}\right)$ ). ${ }^{[19]}$ This can be further underlined by comparison of absolute diffusion coefficients (before normalization; the complete list of diffusion coefficients for all references and solvents is given in the appendix: Table 5-3) which were significantly lower for DMSO and cyclohexane within the defined measurement setup than for other solvents. Hence, to investigate, if changes in viscosity were the cause for the aberrant behavior of CS compounds in these solvents, temperature-dependent measurements were performed exemplary for DMSO- $d_{6} \cdot{ }^{a}$ Upon heating, the normalized diffusion coefficients did not change significantly (see Figure 2-6 (left); the complete set of data for the measurements at different temperatures in DMSO is given in the appendix: Table 5-6; cooling was not an option $\left.\left(\mathrm{mp}\left(\mathrm{DMSO}-d_{6}\right)=20.2^{\circ} \mathrm{C} ; \mathrm{mp}\left(\mathrm{C}_{6} \mathrm{D}_{12}\right)=7{ }^{\circ} \mathrm{C}\right)^{[78]}\right)$. The logarithmic diffusion coefficients stayed mostly within the assumed error interval (0.015; see section 2.1.3). With this data, calibration curves were obtained for all investigated temperatures $\left(25^{\circ} \mathrm{C}, 30^{\circ} \mathrm{C}, 40^{\circ} \mathrm{C}, 50^{\circ} \mathrm{C}\right.$ and $60^{\circ} \mathrm{C}$; the complete set of parameters for these ECCs is given in the appendix: Table 5-7). As shown in Figure 2-6 (right), cor. $\mathrm{R}^{2}$ values vary slightly, but independent of temperature changes which was generally presumed for ECCs and can therefore be confirmed (note that TMB was excluded for these ECCs, as it was used as internal reference). While it cannot be dismissed that viscosity plays a role in the determination of diffusion coefficients, it obviously does not explain the elevated deviations in this case.

\footnotetext{
${ }^{a}$ Note that DMSO was used without being dried beforehand and therefore will accommodate varying quantities of water, which could potentially slightly alter the overall viscosities.
} 
Finally, a closer look at the utilized references revealed a problem: A single reference $\left(\mathrm{Si}(\mathrm{OMe})_{4}\right)$ displayed an increased van-der-Waals density (further illustrated in section 2.1.4). After removal of
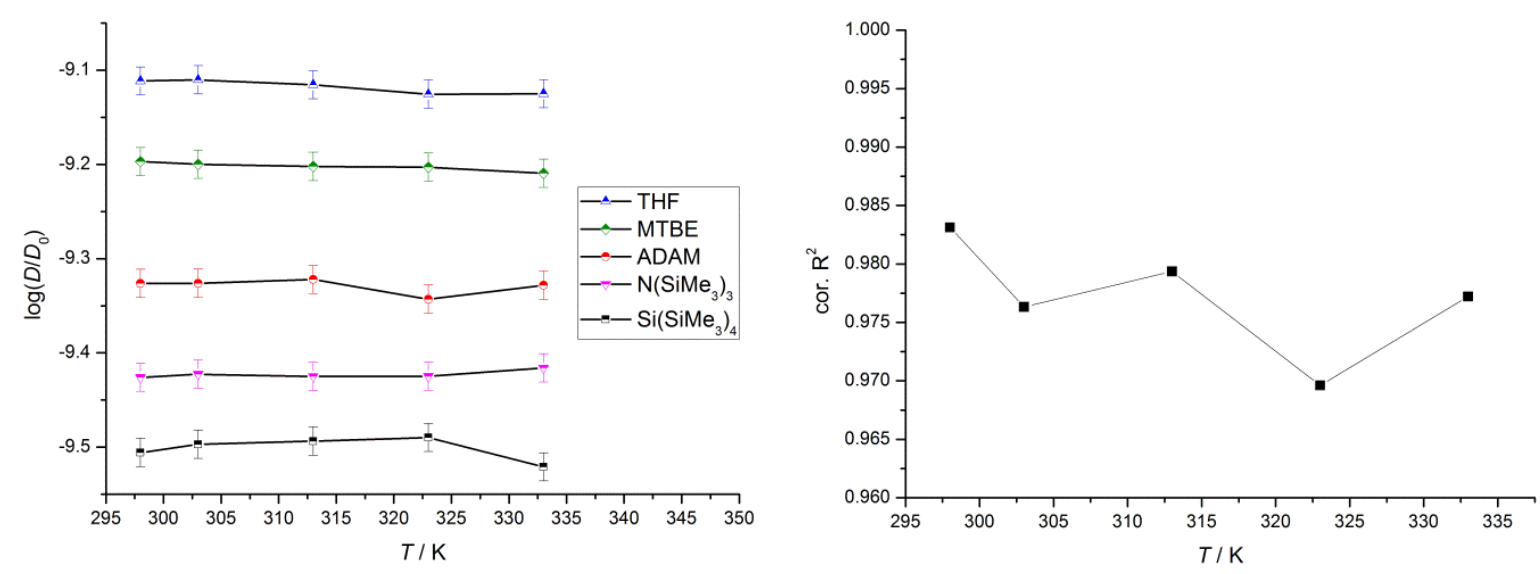

Figure 2-6: Temperature dependence of normalized diffusion coefficients of a selection of CS model compounds in DMSO- $d_{6}$ (left; $25^{\circ} \mathrm{C}$ to $60^{\circ} \mathrm{C}$ ). Most diffusion coefficients only deviate within the assumed error interval. Cor. $\mathrm{R}^{2}$ values of the corresponding ECCs vary independently of temperature (right). ( $D_{0}=1 \mathrm{~m}^{2} / \mathrm{s}$ )

this reference, ${ }^{a}$ the linear fits of compact spherical compounds in DMSO- $d_{6}$ and cyclohexane- $d_{12}$ displayed substantially improved accuracies and cor. $\mathrm{R}^{2}$ values (see Table 2-5). While it should be mentioned that the fit qualities are still a little worse compared to other solvents, the need to differentiate between PCS and NCS compounds in DMSO and cyclohexane has essentially been negated.

Table 2-5: ECC parameters and their errors, av. and max. deviations of back-calculated $M W s$ and cor. $\mathrm{R}^{2}$ values of shape-optimized calibration curves (CS) for DMSO- $d_{6}$ and $\mathrm{C}_{6} \mathrm{D}_{12}$.

\begin{tabular}{cccccccc}
\hline & $\log (K)$ & $\Delta \log (K)$ & $-\alpha$ & $\Delta \alpha$ & av. dev. & max. dev. & cor. $\mathbf{R}^{2}$ \\
\hline Solvent: DMSO- $d_{6}$ & & & & & & & \\
\hline CS & -8.09 & 0.106 & -0.572 & 0.0508 & $\pm 8 \%$ & $\pm 18 \%$ & 0.95 \\
\hline Solvent: $\mathrm{C}_{6} \mathrm{D}_{\mathbf{1 2}}$ & & & & & & & \\
\hline $\mathrm{CS}$ & -7.76 & 0.0877 & -0.572 & 0.0422 & $\pm 8 \%$ & $\pm 15 \%$ & 0.96 \\
\hline
\end{tabular}

In conclusion, the empirical categorization in terms of molecular shapes should be applied with caution. Even though accuracies increase and the correlation between shape and diffusion coefficient is scientifically valid; the categorization itself as well as conclusions thereof can in some cases be prone to bias. It was demonstrated that even a single unsuited reference can alter the findings significantly. Hence, an increase in reference count may help avert this problem in the future, while other approaches towards categorization may also improve the entire methodology and enable prevention of unnecessary bias.

${ }^{a}$ Resulting from its increased van-der-Waals density, $\mathrm{Si}(\mathrm{OMe})_{4}$ was removed from all ECCs herein. 


\subsubsection{Molecule Categorization}

The categorization of model compounds has been a huge improvement of the ECC methodology (see section 2.1.1.2). Still, as already mentioned, this categorization process lacks clear criteria and includes bias. A misplaced or unsuited reference may have negative consequences, as illustrated in the previous section. Hence, to optimize this categorization process, parameters need to be utilized that are accessible theoretically or experimentally and furthermore related to the individual molecular shape. There are many possibilities for such shape-related parameters, i.e. molecular sizes and relations obtained from crystal structure analysis or theoretical calculations. ${ }^{\mathrm{a}}$

In this thesis, the approach of calculating principal moments of inertia $\left(I_{\mathrm{xx}}, I_{\mathrm{yy}}, I_{\mathrm{zz}}\right)$ of the different model compounds was pursued. Since diffusion experiments were performed in isotropic environments, the molecules can rotate freely (molecular tumbling). This rotation is largely determined by the mass and size of the particle as well as its interactions with the solvent. ${ }^{\mathrm{b}}$ Hence, principle moments of inertia should be able to express these criteria and delimit molecular shapes. For example, toluene is flat but comparably small (and light) and can therefore rotate faster than larger, similarly flat compounds. It was previously categorized as DSE instead of ED which might therefore be recognizable utilizing principal moments of inertia. The principal moments of inertia are the elements of the principal inertia matrix (I) (2-6), for which $0 \neq I_{\mathrm{xx}} \leq I_{\mathrm{yy}} \leq I_{\mathrm{zz}}$ applies.

$$
\mathbf{I}=\left[\begin{array}{ccc}
I_{\mathrm{xx}} & 0 & 0 \\
0 & I_{\mathrm{yy}} & 0 \\
0 & 0 & I_{\mathrm{zz}}
\end{array}\right]
$$

The matrix elements can also be used to calculate the relative shape anisotropy $\kappa^{2}$, as shown in formula $(2-7):^{[121]}$

$$
\kappa^{2}=1-\frac{3\left(I_{\mathrm{xx}} I_{\mathrm{yy}}+I_{\mathrm{xx}} I_{\mathrm{zz}}+I_{\mathrm{yy}} I_{\mathrm{zz}}\right)}{\left(I_{\mathrm{xx}}+I_{\mathrm{yy}}+I_{\mathrm{zz}}\right)^{2}}
$$

This relative shape anisotropy $\kappa^{2}$ is always between zero and one. If it is zero, all points are spherically symmetrically distributed and if it is one, they are all located on a line. Hence, this is a good parameter to discuss molecular shapes, especially to differentiate linear from spherical molecules.

Principal moments of inertia were calculated for all model compounds utilizing the program PMIFST $^{[122]}$ with force-field (MMFF94) optimized structures (see Figure 2-7) which produce reasonably good geometries to give a proof of concept.

\footnotetext{
${ }^{a}$ Nowadays, very elaborate parameters can be calculated, e.g. by employing spherical harmonics. ${ }^{[20]}$

${ }^{\mathrm{b}}$ For high concentrations, even solute-solute interactions may have to be considered.
} 
From these principal moments, relative shape anisotropies $\kappa^{2}$ were calculated according to equation (2-7). The complete set of principal moments of inertia as well as their relations and shape anisotropies are given in the appendix (see Table 5-2). In Table 2-6 a limited selection is shown.

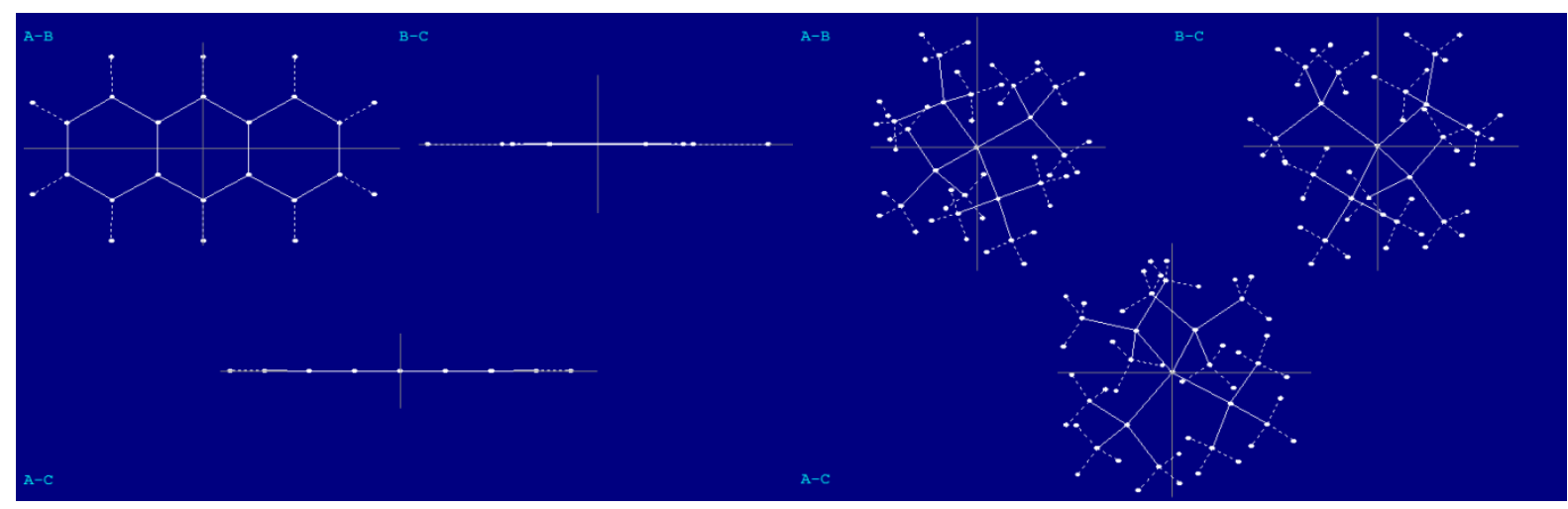

Figure 2-7: Principal axes defined by PMIFST for the calculation of principal moments of inertia of anthracene (left) and $\mathrm{Si}\left(\mathrm{SiMe}_{3}\right)_{4}$ (right).

Table 2-6: Principal moments of inertia calculated with PMIFST ${ }^{[122]}$ for geometry optimized (MMFF94) structures of model compounds used in ECCs. The relative shape anisotropy $\kappa^{2}$ is also listed for each model compound.

\begin{tabular}{|c|c|c|c|c|c|}
\hline Compound & $I_{\mathrm{xx}}\left[\mathrm{u} \AA^{2}\right]$ & $I_{\mathrm{yy}}\left[\mathbf{u} \AA^{2}\right]$ & $I_{z z}\left[\mathbf{u} \AA^{2}\right]$ & $\sim I_{\mathrm{Xx}}: I_{\mathrm{yy}}: I_{\mathrm{zz}}$ & $\kappa^{2} \cdot 100 \%$ \\
\hline 1,3-Indanedione & 301 & 408 & 707 & $1: 1: 2$ & 6.6 \\
\hline 2-PhP & 173 & 905 & 1053 & $1: 5: 6$ & 14.7 \\
\hline 9-MA & 356 & 1133 & 1485 & $1: 3: 4$ & 11.3 \\
\hline Anthracene & 230 & 1113 & 1343 & $1: 5: 6$ & 14.4 \\
\hline Benzene & 89 & 89 & 177 & $1: 1: 2$ & 6.1 \\
\hline DHBP & 1146 & 1890 & 2716 & $1: 2: 2$ & 5.6 \\
\hline Cyclopentane & 76 & 76 & 132 & $1: 1: 3$ & 3.9 \\
\hline DPS & 426 & 1217 & 1425 & $1: 3: 3$ & 8.9 \\
\hline Hexaphenyltrisiloxane & 6123 & 7683 & 8920 & $1: 1: 1$ & 1.1 \\
\hline$i \operatorname{Pr}_{2} \mathrm{O}$ & 122 & 326 & 370 & $1: 3: 3$ & 7.9 \\
\hline Naphthalene & 160 & 409 & 568 & $1: 3: 4$ & 9.8 \\
\hline $\mathrm{N}\left(\mathrm{SiMe}_{3}\right)_{3}$ & 893 & 893 & 1311 & $1: 1: 1$ & 1.8 \\
\hline Pyrene & 490 & 904 & 1394 & $1: 2: 3$ & 7.9 \\
\hline $\mathrm{Si}\left(\mathrm{SiMe}_{3}\right)_{4}$ & 1914 & 1914 & 1914 & $1: 1: 1$ & 0 \\
\hline TPhN & 2876 & 4349 & 6524 & $1: 2: 2$ & 5.4 \\
\hline THF & 70 & 72 & 126 & $1: 1: 2$ & 4.2 \\
\hline TMB & 219 & 510 & 515 & $1: 2: 2$ & 5.6 \\
\hline TMS & 162 & 162 & 162 & $1: 1: 1$ & 0 \\
\hline Toluene & 92 & 199 & 288 & $1: 2: 3$ & 8.6 \\
\hline Triphenylene & 990 & 990 & 1980 & $1: 1: 2$ & 6.3 \\
\hline
\end{tabular}

Two attempts were made to categorize the model compounds utilizing the calculated data: First, model compounds were grouped together that exhibited similar ratios of their principal moments of inertia $\left(I_{\mathrm{xx}}: I_{\mathrm{yy}}: I_{\mathrm{zz}}\right)$ which resulted in three categories: Molecules with the same or similar principal moments for all directions $\left(I_{\mathrm{xx}} \approx I_{\mathrm{yy}} \approx I_{\mathrm{zz}}\right)$ and molecules with the same or similar principal moments for two directions $\left(I_{\mathrm{xx}}<I_{\mathrm{yy}} \approx I_{\mathrm{zz}}\right.$ and $\left.I_{\mathrm{xx}} \approx I_{\mathrm{yy}}<I_{\mathrm{zz}}\right)$. Some model compounds fit the expectations, e.g. TMS and $\mathrm{Si}\left(\mathrm{SiMe}_{3}\right)_{4}$ display $I_{\mathrm{xx}} \approx I_{\mathrm{yy}} \approx I_{\mathrm{zz}}$ and are therefore to be classified as spherical. Others may not be as easily classifiable with this approach, e.g. hexamethyldisilazane which also has similar principal moments in all three dimensions, but was previously assumed to be a dissipated sphere. Additionally, these molecules were categorized depending on their relative 
shape anisotropies $\left(\kappa^{2} \cdot 100 \%\right)$ which were differentiated once again into three categories: Molecules displaying anisotropies of $0-5 \%, 5-10 \%$ and more than $10 \%$. While these shape anisotropies are helpful in differentiating linear from spherical compounds, the minute differences of the model compounds herein were not as easily distinguishable utilizing this parameter. The findings are presented exemplary for benzene- $d_{6}$ in Figure 2-8.
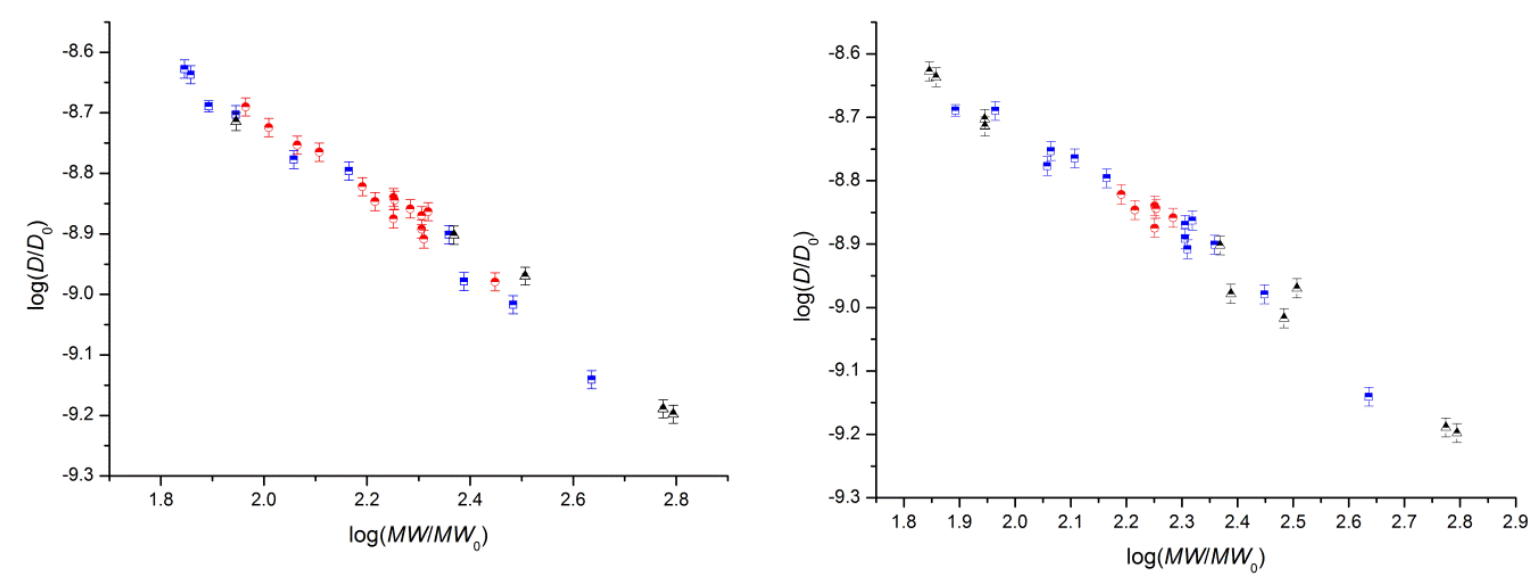

Figure 2-8: Categorization attempts using principle moments of inertia of all model compounds for benzene- $d_{6}$. On the left, molecules are divided by the relations of their principle moments of inertia (black $=I_{\mathrm{xx}} \approx I_{\mathrm{yy}} \approx I_{\mathrm{zz}}$; red $=I_{\mathrm{xx}} \approx I_{\mathrm{yy}} \neq$ $I_{\mathrm{zz}}$; blue $\left.=I_{\mathrm{xx}} \neq I_{\mathrm{yy}} \approx I_{\mathrm{zz}}\right)$. On the right, the same molecules are categorized by their relative shape anisotropies $\kappa^{2}(\mathrm{black}=$ $0-5 \%$; red $=5-10 \%$ and blue $=10 \%+) .\left(D_{0}=1 \mathrm{~m}^{2} / \mathrm{s} ; M W_{0}=1 \mathrm{~g} / \mathrm{mol}\right)$

Linear fits of these categorization attempts led to cor. $\mathrm{R}^{2}$ values similar to merged ECCs (e.g. for compounds with $I_{\mathrm{xx}} \approx I_{\mathrm{yy}} \approx I_{z z}$ : cor. $\mathrm{R}^{2}=0.97$; worst for compounds with $\kappa^{2}=5-10 \%$ : cor. $\mathrm{R}^{2}=0.31$ ). The best result was received for compounds with $\kappa^{2}=0-5 \%$ (cor. $\left.\mathrm{R}^{2}=0.98\right)$ which shows that at least a differentiation into spherical and linear compounds seems feasible using shape anisotropies. This could also help to establish better merged ECCs by simply ruling out the very spherical or very linear molecules. However, the categorization attempts would overall, according to the cor. $\mathrm{R}^{2}$ values, not result in a more accurate $M W$ estimation. This is unfortunate, since the goal of a straightforward categorization procedure could not be accomplished using this route.

It was also difficult to find suitable limits for the categorization of molecules utilizing not only the ratios of principal moments of inertia, but also their absolute values, e.g. THF and cyclopentane have principal moments that behave like $I_{\mathrm{xx}} \approx I_{\mathrm{yy}} \neq I_{\mathrm{zz}}$, however, the absolute principal moments are also very small. The observed diffusion coefficients of these molecules were reduced and they were therefore previously empirically categorized as CS compounds, for which $I_{\mathrm{xx}} \approx I_{\mathrm{yy}} \approx I_{\mathrm{zz}}$ would be assumed. Hence, in the future, a differentiation utilizing absolute principal moments of inertia may be worthwhile, since these incorporate the size of the solute and therefore enable a more accurate classification, e.g. naphthalene has ratios of principal moments similar to anthracene, whereas their absolute values are only half. However, this approach would most certainly require careful evaluation for every compound, therefore also further complicate a desired simple categorization process. Such individual differentiations may also be aided by the calculation of the gyration tensor (S) (2-8) which is purely geometry-based, i.e. it only depends on the angles and bond lengths of the 
investigated molecule. Also, the gyration tensor should be very similar to the inertia tensor in the absence of very heavy elements. The elements of this gyration tensor can be used to calculate the asphericity $b$ and the acyclindricity $c$ shown in formulae (2-9) and (2-10), respectively which can be further criteria for a more elaborate categorization.

$$
\begin{gathered}
\mathbf{S}=\left[\begin{array}{ccc}
S_{\mathrm{xx}} & 0 & 0 \\
0 & S_{\mathrm{yy}} & 0 \\
0 & 0 & S_{\mathrm{zz}}
\end{array}\right] \\
b=S_{\mathrm{zz}}-\frac{1}{2}\left(S_{\mathrm{xx}}+S_{\mathrm{yy}}\right) \\
c=S_{\mathrm{yy}}-S_{\mathrm{xx}}
\end{gathered}
$$

\subsubsection{Theoretical Error Analysis ${ }^{\mathrm{a}}$}

Deviations of back-calculated $M W$ s correspond to an empirical error limit of the ECCs, but cannot replace a theoretical error calculation. So far, this subject has scarcely been touched in preceding studies. Equation (2-11) incorporates all parameters to predict $M W_{\text {det: }}$ :

$$
M W_{\text {det }}=\left(\frac{D_{\mathrm{x}} D_{\mathrm{ref}, \mathrm{fix}}}{D_{\mathrm{ref}} K}\right)^{-\frac{1}{\alpha}}
$$

The diffusion coefficients $\left(D_{\mathrm{x}}, D_{\text {ref,fix }}\right.$ and $\left.D_{\text {ref }}\right)$ used therein are clearly error-prone as well as the linear fit parameters $-\alpha$ and $K$ of the ECCs. Error propagation of formula (2-11) resulted in equations (2-12) and (2-13) for the calculation of the maximum error of the predicted $M W_{\text {det }}$ $\left(\Delta M W_{\text {det }}\right)$ and the relative error $\left(\Delta M W_{\text {det,rel }}\right)$, respectively:

$$
\begin{gathered}
\Delta M W_{\text {det }}=\sqrt{\left(\frac{M W_{\text {det }}}{D_{\text {ref }} \alpha} \cdot \Delta D_{\text {ref }}\right)^{2}+\left(\frac{M W_{\text {det }}}{K \alpha} \cdot \Delta K\right)^{2}+\left(-\frac{M W_{\text {det }}}{D_{\mathrm{x}} \alpha} \cdot \Delta D_{\mathrm{x}}\right)^{2}} \\
+\left(-\frac{M W_{\text {det }}}{D_{\text {ref,fix }} \alpha} \cdot \Delta D_{\text {ref, fix }}\right)^{2}+\left(-\frac{M W_{\text {det }} l o g\left(\frac{D_{\mathrm{x}} D_{\text {ref,fix }}}{D_{\text {ref }} K}\right)}{\alpha^{2}} \cdot \Delta \alpha\right)^{2} \\
\Delta M W_{\text {det, rel }}=\frac{\Delta M W_{\text {det }}}{M W_{\text {det }}} \cdot 100 \%
\end{gathered}
$$

The errors for the linear fit parameters $(\Delta \alpha$ and $\Delta \log (K))$ are listed further above (Table 2-3, Table 2-4 and Table 2-5). These errors are taken from the least-squares fitting procedure as implemented in the program Origin Pro 8.5G. Errors for the diffusion coefficients were estimated from multiple DOSY measurements which resulted in a maximum variation of $\log (D)$ values of about $0.0075 . \Delta \log (D)$ was defined as twice this deviation (0.015) and $\Delta D$ were calculated relatively using equation (2-14) for analytes and internal references ( $\Delta K$ was calculated likewise).

a The theoretical error analysis was not featured, but first presented in: S. Bachmann, B. Gernert, D. Stalke, Chem. Commun. 2016, 52, 12861-12864. ${ }^{[4]}$ 


$$
\Delta D=D\left(\frac{\Delta \log (D)}{\log (D)}\right) \cdot 100 \%
$$

However, this leads to smaller relative errors for smaller diffusion coefficients, i.e. smaller aggregates. Still, there is only a difference of about $1 \%$ in the final errors which seems to be negligible. The average errors for all ECCs are given in Table 2-7.

Table 2-7: Average relative theoretical errors $\left(\Delta M W_{\text {det,rel }}\right)$ for all solvents and merged as well as shape-optimized ECCs.

\begin{tabular}{|c|c|c|c|c|}
\hline & $\Delta M W_{\text {det,rel }}$ & $\Delta M W_{\text {det,rel }}$ & $\Delta M W_{\text {det,rel }}$ & $\Delta M W_{\text {det,rel }}$ \\
\hline Solvent & Merged & DSE & ED & CS \\
\hline $\mathrm{CDCl}_{3}$ & $\pm 10 \%$ & $\pm 12 \%$ & $\pm 18 \%$ & $\pm 10 \%$ \\
\hline $\mathrm{CD}_{2} \mathrm{Cl}_{2}$ & $\pm 9 \%$ & $\pm 8 \%$ & $\pm 15 \%$ & $\pm 8 \%$ \\
\hline $\mathrm{C}_{6} \mathrm{D}_{6}$ & $\pm 7 \%$ & $\pm 5 \%$ & $\pm 11 \%$ & $\pm 11 \%$ \\
\hline $\mathrm{CD}_{3} \mathrm{OD}$ & $\pm 9 \%$ & $\pm 10 \%$ & $\pm 7 \%$ & $\pm 15 \%$ \\
\hline $\mathrm{CD}_{3} \mathrm{CN}$ & $\pm 8 \%$ & $\pm 9 \%$ & $\pm 12 \%$ & $\pm 15 \%$ \\
\hline THF- $d_{8}$ & $\pm 7 \%$ & $\pm 5 \%$ & $\pm 6 \%$ & $\pm 8 \%$ \\
\hline Toluene- $d_{8}$ & $\pm 7 \%$ & $\pm 4 \%$ & $\pm 9 \%$ & $\pm 9 \%$ \\
\hline DMSO- $d_{6}$ & $\pm 8 \%$ & $\pm 7 \%$ & $\pm 8 \%$ & $\pm 19 \%$ \\
\hline $\mathrm{C}_{6} \mathrm{D}_{12}$ & $\pm 13 \%$ & $\pm 9 \%$ & $\pm 11 \%$ & $\pm 15 \%$ \\
\hline
\end{tabular}

The complete set of all individual, calculated $\Delta M W_{\text {det,rel }}$ for all references and solvents is given in the appendix (Table 5-22 (merged ECCs); Table 5-23 (shape-optimized ECCs)).

The theoretical errors $\left(\Delta M W_{\text {det }}\right)$ are mostly influenced by the errors of the linear fit parameters themselves $(\Delta \log (K)$ and $\Delta \alpha)$. Their contributions make up about $99 \%$ of the final error. The limitation of the technique is therefore closely related to the quality and validity of the ECCs, i.e. the validity of the power-law. Still, the presented theoretical errors seem to be an adequate measure of how accurate the $M W$ prediction can effectively be. Essentially, this would be 4 to $19 \%$ which matches the previously discussed empirical deviations attained from back-calculated $M W$ s.

For the merged ECCs the theoretical limitations are mostly exceeded by their previously discussed empirical max. deviations (e.g. for DMSO- $d_{6}$ : max. dev. (merge) $= \pm 20 \%, \Delta M W_{\text {det,rel }}($ merge $)= \pm 8 \%$ ). This is on the one hand encouraging, as the methodology is not overstating the data, but on the other hand a clear indication that there is room for improvement. Such improvements have been attempted by introducing the categorization of compounds in relation to their shape (see section 2.1.1.2). The theoretical errors of the resulting shape-optimized ECCs are comparable to those of merged ECCs, while their empirical deviations are substantially diminished. Empirical deviations are in some cases even better than the theoretical limitations. Hence, an overinterpretation of the data is a possibility and in such cases, theoretical limitations should be taken into account instead of the empirical deviations. Furthermore, the theoretical error calculation can be an indicator towards the overall quality of ECCs, e.g. the high theoretical error for the CS ECC of DMSO- $d_{6}$ shows that the categorization might in this case still be improved. 


\subsubsection{Heavy Atoms in the ECC-DOSY-MW Estimation}

There is another issue which is of great importance for the interpretation of results obtained from ECC-DOSY: Molecular sizes (volumes) can mostly be correlated proportionally to molecular weights (see Figure 2-9). This works well, if only hydrocarbons with an occasional heavier atom are compared. But, there are some cases where the increase in size is no longer correlated to the increase in weight. For example, deuterium atoms are roughly the same size as hydrogen atoms, whereas their weights are doubled. Thus, in the ECC-MW estimation deuterated and hydrogenated molecules cannot be distinguished, since their diffusion coefficients are almost the same in solution as these are mainly dependent on their quasi-identical molecular sizes. For this reason, all hypothetical aggregates herein are only proposed to be coordinated by hydrogenated donors, even though in deuterated solvents this does not have to be the case.

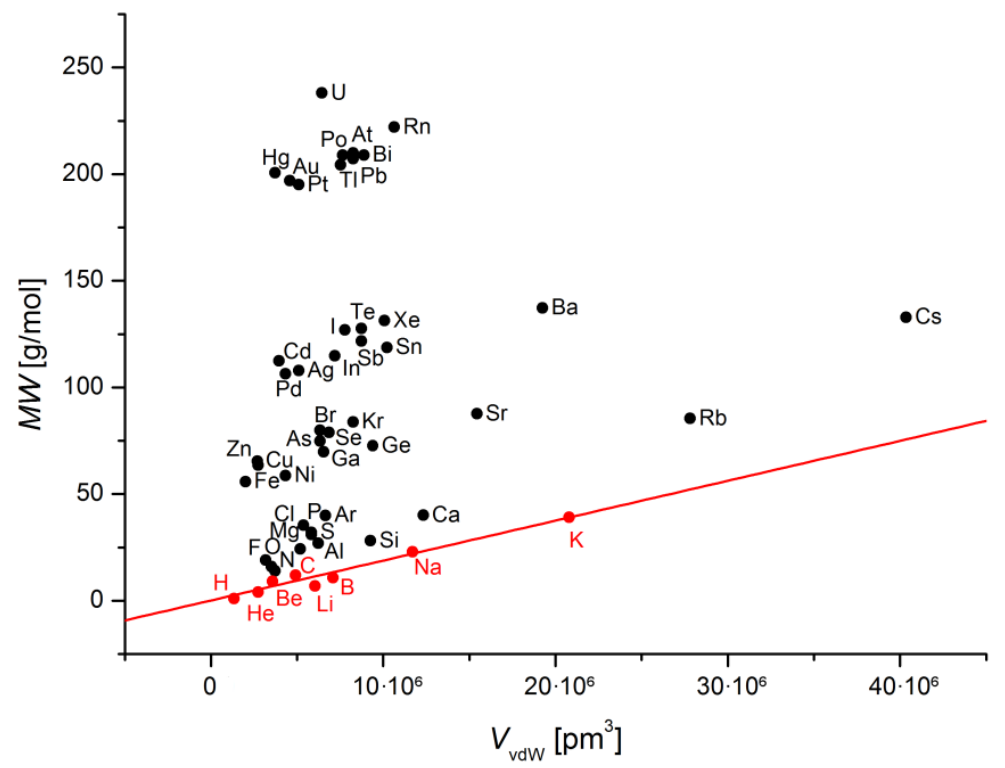

Figure 2-9: Plot of the molecular weight $(M W)$ of atoms against their van-der-Waals volumes $\left(V_{\mathrm{vdw}}=\frac{\mathbf{4}}{\mathbf{3}} \boldsymbol{\pi} \boldsymbol{r}_{\mathrm{vdw}}{ }_{\mathrm{vdw}}\right)$. The red line demonstrates for which elements (also marked red) a linear dependence can be assumed for the ECCs herein. Other elements (e.g. heavier halides like Br or transition metals like Cd) deviate strongly.

This is problematic for heavier elements (e.g. transition metals or higher halides) which invoke the same problem. The diffusion coefficients of molecules incorporating such elements are normally higher than that of molecules of the same mass, but without heavier elements and their molecular weights would be underestimated. The influence of these elements gets weaker as the molecules get larger, e.g. a bromine atom has a heavier impact on falsifying diffusion coefficients of a small molecule, since the effect depends on the bromine's percentage of the overall mass. One way to compensate for this is to establish specialized calibration curves with molecules that incorporate the same heavy elements or other similar irregularities as was done by Wang and Pedersen et al.. ${ }^{[13], \text { a }}$ However, it is only viable if certain molecular properties are known a priori, e.g. the number of

${ }^{\text {a }}$ Calculations of $M D_{\mathrm{W}}$ for their used molecules can be found in the appendix (see Table 5-25). 
heavy atoms. Still, utilizing this procedure of specialized ECCs is valid. However, by recognizing these problems beforehand they can be circumnavigated or used to improve the whole methodology. Our group could previously show that such irregularities can be recognized by simply calculating the so-called molar van-der-Waals density $\left(M D_{\mathrm{w}}\right.$; see equation (2-15)) of proposed aggregates or utilized references. ${ }^{[109]}$

$$
M D_{\mathrm{W}}=\frac{M W}{\sum V_{\mathrm{vdW}}}=\frac{M W}{\sum_{i=1}^{n} \frac{4}{3} \pi r_{v d W, i}^{3}}
$$

If $M D_{\mathrm{W}}$ values are between 4.2 and $5.9 \cdot 10^{29} \mathrm{~g} /\left(\mathrm{mol} \cdot \mathrm{m}^{3}\right), M W \mathrm{~s}$ can be safely predicted by the ECCs given herein, as the corresponding aggregates do either not contain any heavy elements or their effects are negated by the fact that the molecules are of considerable size.

The sum of the van-der-Waals volumes of all individual atoms $\left(V_{\mathrm{vdW}}\right)$ of a molecule is the denominator, when calculating $M D_{\mathrm{w}}$. Van-der-Waals volumes of a selection of elements are given in Table 2-8.

Table 2-8: Van-der-Waals volumes ( $\left.V_{\mathrm{vdw}}\right)$ of a selection of elements in increasing order. Van-der-Waals radii $\left(r_{\mathrm{vdw}}\right)$ were taken from references [123].

\begin{tabular}{llllllllllll}
\hline Element & $\mathrm{H}$ & $\mathrm{Zn}$ & $\mathrm{F}$ & $\mathrm{O}$ & $\mathrm{N}$ & $\mathrm{Cd}$ & $\mathrm{C}$ & $\mathrm{Mg}$ & $\mathrm{Cl}$ & $\mathrm{P}$ & $\mathrm{S}$ \\
\hline $\boldsymbol{V}_{\mathbf{v d w}}\left[\mathbf{m}^{\mathbf{3}} \cdot \mathbf{1 0}^{-30}\right]$ & 5.6 & 11.3 & 13.3 & 14.7 & 15.6 & 16.5 & 20.6 & 21.7 & 22.5 & 24.4 & 24.4 \\
\hline Element & $\mathrm{Li}$ & $\mathrm{Br}$ & $\mathrm{B}$ & $\mathrm{Si}$ & $\mathrm{Na}$ & $\mathrm{K}$ & $\mathrm{Rb}$ & $\mathrm{Cs}$ & & & \\
\hline $\boldsymbol{V}_{\text {vdw }}\left[\mathbf{m}^{\mathbf{3}} \cdot \mathbf{1 0}^{-30}\right]$ & 25.3 & 26.5 & 29.7 & 38.8 & 49.0 & 87.1 & 116.5 & 169.0 & & \\
\hline
\end{tabular}

To exclude related discrepancies, $M D_{\mathrm{w}}$ were calculated for all herein proposed aggregates as well as model compounds (see Table 5-24). All molecules that inhibit higher $M D_{\mathrm{W}}$ were dismissed in the preparation of ECCs (e.g. $\mathrm{CDCl}_{3}$, DMSO or $\left.\mathrm{Si}(\mathrm{OMe})_{4}\right)$.

$M D_{\mathrm{W}}$ enabled other options to correct corresponding deviations within the $M W$ estimation process aside from establishing the aforementioned specialized ECCs, i.e. deviations of $M W s$ or diffusion coefficients can simply be correlated to the respective molar van-der-Waals densities of molecules that inhibit heavier atoms to generate a correctional factor. This was first explained by Neufeld: ${ }^{[109 b]}$ The estimated $M W$ s of molecules with elevated $M D_{\mathrm{w}}$ were corrected to what they should have been for the merged ECCs depending on their diffusion coefficients. The ratio of these corrected and the uncorrected $M W$ s (correction factor) was plotted against the respective molecule's $M D_{\mathrm{W}}$ which resulted in a seemingly linear dependency that could be fitted accordingly. However, due to the limited reference count, this result was ultimately not very reliable.

Therefore, the following results were produced in cooperation with Kreyenschmidt ${ }^{[124]}$ with a master thesis (for more detailed information see reference ${ }^{[124]}$ ): To cure the lack of limited references, 60 different mono- or multibrominated and -iodated molecules were measured that also exhibited

${ }^{a}$ Manuscript in preparation. ${ }^{[5]}$ 
elevated $M D_{\mathrm{W}}$. With this data, a similar approach to that of Neufeld was chosen, i.e. to correct diffusion coefficients and plot the ratio of the normalized uncorrected and corrected diffusion coefficients against the $M D_{\mathrm{w}}$. Instead of obtaining the previously anticipated linear dependence a non-linear correlation became apparent due to the increased reference count and could be fitted adequately (see equation (2-16); see Figure 2-10). This was done for THF- $d_{8}$ and benzene- $d_{6}$. Unfortunately, the additional parameters $(a, k$ and $x c)$ are only identified from distribution and do not have any physical basis, yet.

$$
X_{\text {cor }}=\frac{\log \left(D_{\text {merge }}\right)}{\log \left(D_{\mathrm{x}, \text { norm }}\right)}=\frac{a}{\left(1+e^{\left(-k\left(M D_{\mathrm{W}}-x c\right)\right)}\right)}
$$

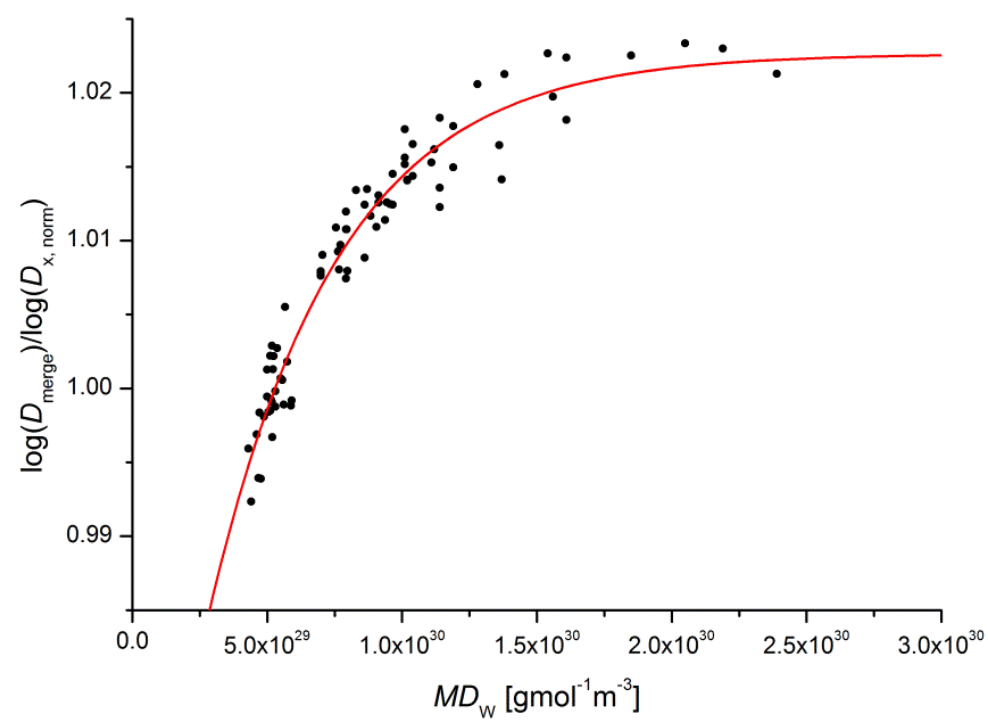

Figure 2-10: Non-linear fit of a plot of $\log \left(D_{\text {merge }}\right) / \log \left(D_{\mathrm{x}, \text { norm }}\right)$ against $M D_{\mathrm{W}}$ in THF- $d_{8}$.

Here, $D_{\text {merge }}$ are the corrected diffusion coefficients assumed for aggregates of the same $M W$ without heavy atoms on the corresponding merged ECC. The correctional factor $\left(X_{\text {cor }}\right)$ seems to approach a limiting value with increasing $M D_{\mathrm{w}}$. While there need to be further investigation into this behavior, it can be noted that molecules that display high $M D_{\mathrm{w}}$ are comparably small with high heavy atom content, e.g. $M D_{\mathrm{W}}\left(\mathrm{CHBr}_{3}\right)=2.39 \cdot 10^{30} \mathrm{~g} /\left(\mathrm{mol} \cdot \mathrm{m}^{3}\right) ; M D_{\mathrm{W}}\left(\mathrm{SiHBr}_{3}\right)=2.17 \cdot 10^{30} \mathrm{~g} /\left(\mathrm{mol} \cdot \mathrm{m}^{3}\right)$; $M D_{\mathrm{W}}\left(\mathrm{C}_{2} \mathrm{H}_{4} \mathrm{I}_{2}\right)=2.19 \cdot 10^{30} \mathrm{~g} /\left(\mathrm{mol} \cdot \mathrm{m}^{3}\right)$.

Using the parameters of the fit curves (THF- $d_{8}: a=1.023 \pm 0.001, k=(2.156 \pm 0.188) \cdot 10^{-30}, x c=$ $(-1.228 \pm 0.137) \cdot 10^{30}$; benzene- $d_{6}: a=1.028 \pm 0.001, k=(1.799 \pm 0.155) \cdot 10^{-30}, x c=(-1.495 \pm$ $0.155) \cdot 10^{30}$ ) the normalized diffusion coefficients of compounds with elevated $M D_{\mathrm{W}}$ can be corrected in dependence of their $M D_{\mathrm{W}}$ by simply multiplying them with their individual correction factor $X_{\text {cor }}$. While the correction factor seems to have a narrow range $\left(0.99<X_{\text {cor }}<1.025\right)$, the correction does in almost all cases exceed the theoretical variation of diffusion coefficients (0.015).

Afterwards, the corrected diffusion coefficients can be used in a classical ECC-DOSY-MW estimation with merged calibration curves of the respective solvents. Note that the fit curve 
parameters have to be improved at the same time as the merged ECCs are improved, since they are closely related. Using this technique, $M W$ s were calculated with highly improved accuracy for molecules with a $M D_{\mathrm{w}}$ of $5.6 \cdot 10^{29}$ to $2.4 \cdot 10^{30} \mathrm{~g} /\left(\mathrm{mol} \cdot \mathrm{m}^{3}\right)$. Unfortunately, just using this approach, a better $M W$ prediction of molecules that do not have elevated $M D_{\mathrm{W}}$ was not achieved and therefore this procedure does not need to be applied in such cases. Furthermore, due to the incorporation of this additional empirical fit to the methodology, the theoretical errors would certainly rise and it is therefore doubtful that a $M W$ estimation should be any better than 10 to $20 \%$ as is reflected by the maximum empirical deviations (THF- $d_{8}$ : av. dev. $= \pm 6 \%$; max. dev. $= \pm 23 \%$; benzene- $d_{6}$ : av. dev. $= \pm 4 \%$; max. dev. $= \pm 13 \%$ ).

\subsubsection{MW Estimation Software ${ }^{a}$}

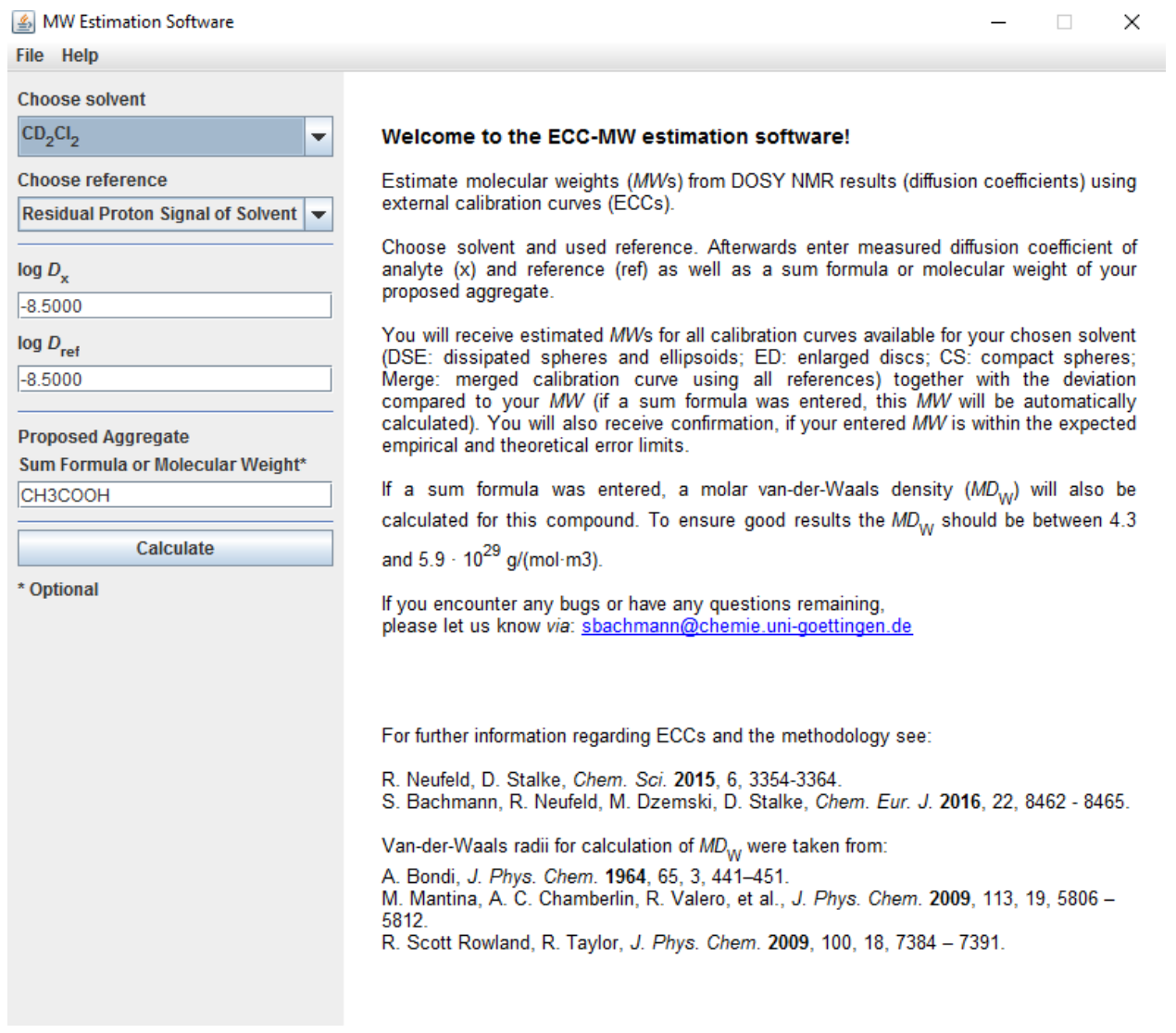

Figure 2-11: Screenshot of the starting screen displaying the welcoming message of the ECC-MW estimation software along with its general graphical user interface (GUI).

To address a larger number of researchers, an easy to use yet comprehensive software was programmed that incorporates state-of-the-art approaches towards ECCs. The software will be continuously updated. Everything that is discussed in this section is related to version 1.0. The

a Some results of this section have been published separately: S. Bachmann, B. Gernert, D. Stalke, Chem. Commun. 2016, 52, 12861-12864. ${ }^{[4]}$ 
newest version is always available for download on: http://www.stalke.chemie.unigoettingen.de/mwestimation/

On startup, the software recognizes the user's system setting and is displayed in the according language (the default setting is English, while German is also available). The idea behind the program is to simplify the $M W$ estimation process and guide the user to ultimately estimate $M W \mathrm{~s}$ systematically and precisely. Therefore, a welcoming message familiarizes the user with the required input and the methodology itself (see Figure 2-11).

Within the main graphical user interface (GUI), the user is asked to choose the used solvent and internal reference and insert logarithmic measured diffusion coefficients of the analyte $\left(\log \left(D_{\mathrm{x}}\right)\right)$ and the internal reference $\left(\log \left(D_{\text {ref }}\right)\right)$. In the options, the input can be changed towards the non-logarithmic form of the diffusion coefficients. Furthermore, users can input an $M W_{\text {calc }}$ or a sum formula of hypothetical aggregates. If a sum formula is entered, $M W_{\text {calc }}$ and $M D_{\mathrm{W}}$ will be automatically calculated. The user will receive verification, if the $M D_{\mathrm{W}}$ is within a reasonable range.

With the entered diffusion values, $M W_{\text {det }}$ will be calculated and displayed for every available ECC of the chosen solvent. $M W_{\text {calc }}$ will be compared to these $M W_{\text {det }}$ i.e. $M W_{\text {dif }}$ will be calculated and displayed. Furthermore, confirmation will be given if the calculated $M W_{\text {dif }}$ is within the empirical and/or theoretical error limit of the respective ECC (see Figure 2-12). The error limits may also be displayed numerically. Additionally, there is an option to change the theoretical errors of the individual diffusion coefficients.

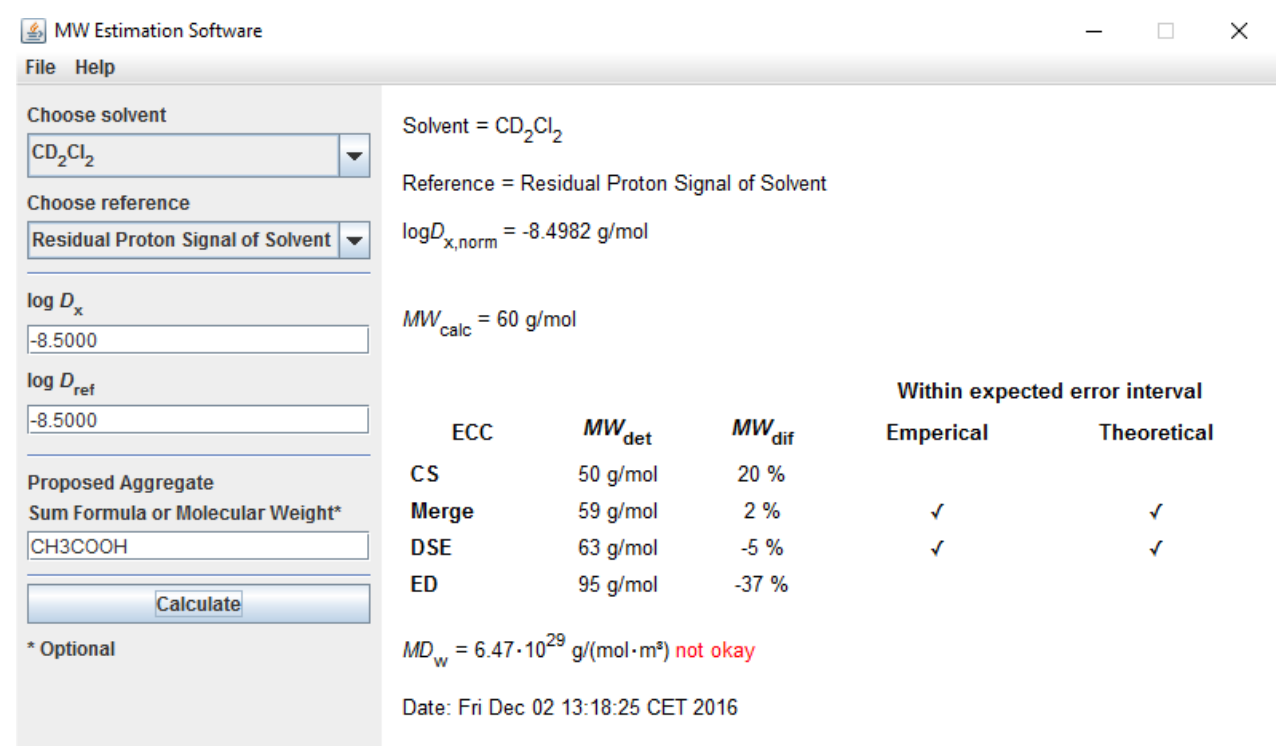

Figure 2-12: Screenshot of a $M W$ estimation. The left-hand side shows the chosen and entered parameters, while the right-hand side shows results, i.e. the normalized diffusion coefficient, the calculated $M W_{\text {calc }}$ from the entered sum formula, the calculated $M W_{\text {det }}$ for every available ECC for the chosen solvent, $M W_{\mathrm{dif}}$ for each ECC, respectively, confirmations if $M W_{\text {dif }}$ is within the theoretical and/or empirical error range, date and time of the estimation and finally a molar van-der-Waals density for the entered sum formula. 
The chosen and entered data will automatically be saved upon closing the program. Furthermore, manually saving and loading this data is an option. Results may also be copied, printed or simply reproduced from saved data to facilitate publication. Within the software a library is given (in form of several .json files) that can easily be changed and new calibration curves as well as references can be added. Moreover, previous ECCs can easily be adapted to new findings.

In the future, further improvements towards the software are envisioned, i.e. correction of diffusion coefficients in relation to $M D_{\mathrm{W}}$ (for information on this and $M D_{\mathrm{W}}$ see section 2.1.4). 


\subsection{Structure Elucidation of Alkali Metal Cyclopentadienide Derivatives via Diffusion NMR}

In the previous chapter, the ECC methodology was reviewed and improvements were made to elevate the degree of reliance. In this chapter, this methodology will be utilized to elucidate the solution state structures of alkali metal cyclopentadienides (CpMs) and their derivatives (MeCpMs and $\mathrm{Cp}^{\star} \mathrm{Ms}$ ). As was described in section 1.1.2, these heavily used organometallic building blocks have been thoroughly investigated in the solid state and were reviewed in many articles. ${ }^{[33]}$ However, information on solution state structures is scarce. Hence, further evidence is needed to finally clarify persistent ambiguities.

\subsubsection{Solution State Structures of Alkali Metal Cyclopentadienides (CpMs) in Different Solvents ${ }^{a}$}

Alkali metal cyclopentadienides ( $\mathrm{CpLi}, \mathrm{CpNa}, \mathrm{CpK}, \mathrm{CpRb}$ and $\mathrm{CpCs}$ ) were synthesized according to literature procedures or in slightly adapted one-step syntheses. These syntheses will not be discussed any further in the main part of this work, but are listed for completeness in section 4.2.1. CpMs were then analyzed fully by $1 \mathrm{D}$ and 2D NMR experiments in different solvent environments.

\subsubsection{Solution State Structures of CpMs in THF- $d_{8}$}

In the solid state CpMs arrange into polymeric chains and can be disaggregated into monomers by donating solvents (for further information see section 1.1.2). These structures are mostly contact ion pairs (CIPs) and provide the basis on which solution structures have to be judged. CpMs have very poor solubilities in hydrocarbons and ethers. THF is the only solvent that provides reasonable concentrations and was therefore chosen for solution state structure analysis. The effects of more strongly donating solvents, i.e. ammonia and DMSO, on the solution state structures of CpMs will be discussed in a subsequent section.

In THF- $d_{8}$, the ${ }^{1} \mathrm{H}$ and ${ }^{13} \mathrm{C}$ NMR spectra of all CpMs show only a single resonance. The signal tends to be shifted towards higher field when descending Group 1 (see Figure 2-13). ${ }^{7} \mathrm{Li}(\delta=-7.75 \mathrm{ppm}$ ) for $\mathrm{CpLi}$ and ${ }^{133} \mathrm{Cs}$ resonances $(\delta=-240.1 \mathrm{ppm})$ for $\mathrm{CpCs}$ were found to be in accordance with literature. ${ }^{[125]}$ The ${ }^{23} \mathrm{Na}$ resonance of $\mathrm{CpNa}(\delta=-28$ to $-31 \mathrm{ppm})$ is shifted towards lower field in solution compared to solid state magic angle spinning (MAS) NMR findings for a mixture of $\mathrm{CpNa} /[\mathrm{CpNa}(\mathrm{THF})]$ and can be shifted even lower upon cooling. ${ }^{[59 \mathrm{~m}]}$

\footnotetext{
${ }^{\text {a }}$ Some results of this section have been published separately: S. Bachmann, B. Gernert, D. Stalke, Chem. Commun. 2016, 52, 12861-12864. ${ }^{[4]}$
} 


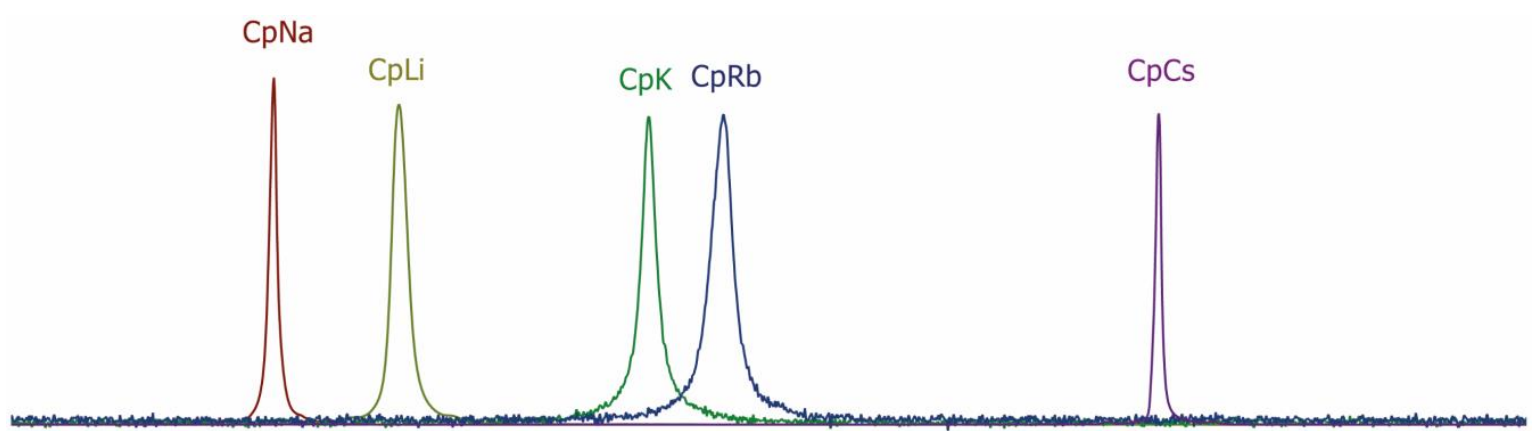

5.74

5.70

5.66

${ }_{\delta / \mathrm{ppm}}^{5.62} 5.58$

5.54

5.50

5.46

Figure 2-13: Superposition of ${ }^{1} \mathrm{H}$ NMR spectra of CpLi, CpNa, CpK, CpRb and CpCs (15 mM solutions, except for CpK and $\mathrm{CpRb}$ which were barely soluble) in THF- $d_{8}$ at $25^{\circ} \mathrm{C}$. A shift towards higher field can be observed for larger alkali metals (exception $\mathrm{CpNa}$ ).

It should be stated that all of these chemical shifts for the alkali metals are quite abnormal for organometallic compounds (e.g. normally the chemical shifts of caesiumorganics range from $\delta=$ 250 to $-60 \mathrm{ppm}) .{ }^{[126]}$ One reason for these unique shifts is the fact that the alkali metals are situated above the $\pi$-plane of the aromatic five-membered cyclopentadienide which provides additional shielding to the metal nuclei. However, such ring currents should only provide a maximum of $10 \mathrm{ppm}$ of shielding independent of observed nucleus.

A similar downfield shift as observed upon cooling for the ${ }^{23} \mathrm{Na}$ resonance of $\mathrm{CpNa}$ was also noticed for the ${ }^{7} \mathrm{Li}$ NMR signal of $\mathrm{CpLi}$ and is further accompanied by broadening (see Figure 2-14).

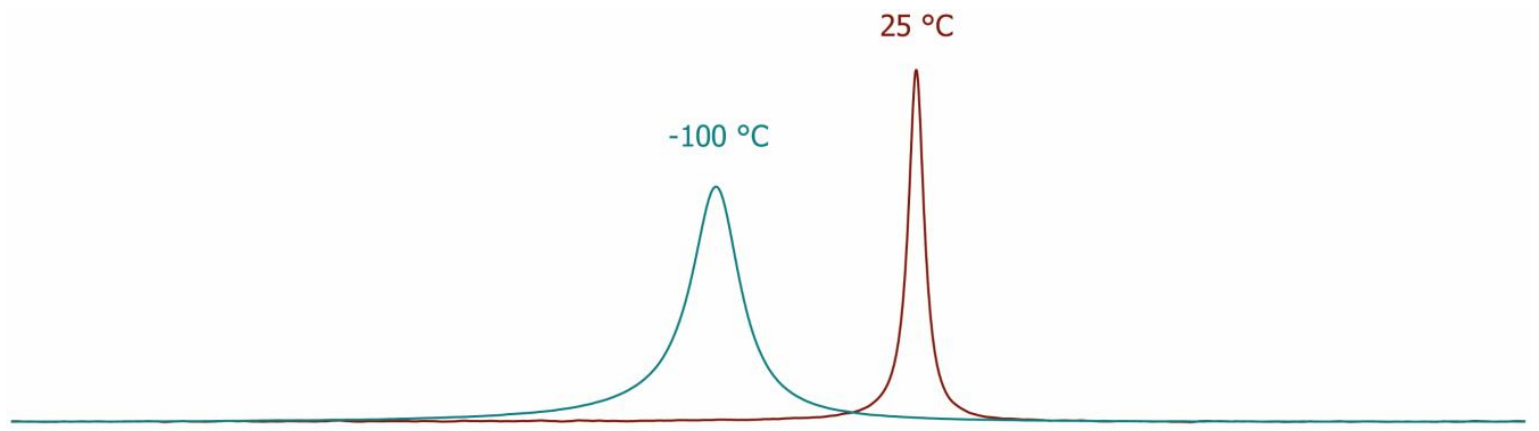

\begin{tabular}{|c|c|c|c|c|c|c|c|c|c|c|}
\hline 4.5 & -5.0 & -5.5 & -6.0 & -6.5 & -7.0 & -7.5 & -8.0 & -8.5 & -9.0 & -9.5 \\
\hline
\end{tabular}

Figure 2-14: Superposition of ${ }^{7} \mathrm{Li}$ NMR spectra of different samples of CpLi at ambient temperature (red) and $-100{ }^{\circ} \mathrm{C}$ (cyan). Broadening and shifting of the resonance are clearly observable.

In 1990, Paquette et al. already reported this behavior. ${ }^{[66]}$ They investigated a closely related compound to $\mathrm{CpLi}$, namely lithium isodicyclopentadienide, and could provide evidence for a fast exchange process between a monomeric and a "sandwich" dimeric species. In the same study, they also investigated CpLi and concluded a similar behavior, based on the splitting of the ${ }^{6} \mathrm{Li}$ NMR signal at lower temperatures (splitting was first observed at $-107^{\circ} \mathrm{C}$ ). Still, they could not "tell 
whether the monomer-dimer equilibrium of" $\mathrm{CpLi}$ "is shifted to either side at room temperature". In this work, $\mathrm{CpLi}$ was cooled to $-100^{\circ} \mathrm{C}$. However, measurements showed no splitting (see Figure 2-14) and at about $-105^{\circ} \mathrm{C} \mathrm{CpLi} \mathrm{precipitated} \mathrm{completely.} \mathrm{Its} \mathrm{behavior} \mathrm{could} \mathrm{therefore} \mathrm{not} \mathrm{be}$ studied further.

Aside from these 1D spectra that provide chemical shift information, DOSY NMR spectroscopy was used to estimate MWs utilizing the ECC methodology. The ECCs for dissipated spheres and ellipsoids (DSE) were found to be quite accurate for determining the MWs of organometallic reagents. ${ }^{[109 a]}$ Therefore, all $M W$ s of CpMs in THF- $d_{8}$ were estimated via the DSE-ECC. For comparison, MWs were also estimated using the merged ECC. The mandatory molar van-der-Waals density calculations have also been done and the complete set of data is given in the appendix (see Table 5-39). However, for all aggregates proposed herein errors due to higher $M D_{\mathrm{W}}$ can be excluded. The estimated $M W_{\text {det }}$ were compared to $M W_{\text {calc }}$ of likely monomeric [CpM(THF) $)_{x}$ and dimeric aggregates $\left[(\mathrm{CpM})_{2}(\mathrm{THF})_{\mathrm{x}}\right]$ with $\mathrm{x}=0-4, \mathrm{M}=\mathrm{Li}, \mathrm{Na}, \mathrm{K}, \mathrm{Rb}, \mathrm{Cs}$ (see Table 2-9; the complete set of data is given in the appendix: Table 5-26 (CpLi); Table 5-28 (CpNa); Table 5-30 (CpK); Table 5-32 (CpRb); Table 5-34 (CpCs)).

Table 2-9: ECC ${ }^{\mathrm{THF}}$ (DSE and merged) were used to predict $M W_{\mathrm{det}}$ of CpMs in THF- $d_{8}$. The accuracy of the ECC ${ }^{\mathrm{THF}}$ (DSE) is in the range of $M W_{\mathrm{dif}}= \pm 8 \%$ and of the $\mathrm{ECC}^{\mathrm{THF}}$ (merged) in the range of $M W_{\mathrm{dif}}= \pm 18 \%$. Theoretical errors as discussed in section 2.1.3 are given as standard deviations for all estimated $M W$ s. All results in this table are from measurements at $25^{\circ} \mathrm{C} . M W_{\mathrm{dif}}$ are given for some exemplary aggregates.

\begin{tabular}{|c|c|c|c|c|c|}
\hline & & & {$\left[\mathrm{CpM}(\mathrm{THF})_{2}\right]$} & {$\left[\mathrm{CpM}(\mathrm{THF})_{3}\right]$} & {$\left[(\mathrm{CpM})_{2}(\mathrm{THF})_{4}\right]$} \\
\hline & $\begin{array}{c}M W_{\mathrm{det}} \\
\text { (merged) }[\mathrm{g} / \mathrm{mol}]\end{array}$ & $\begin{array}{c}M W_{\mathrm{det}} \\
(\mathrm{DSE})[\mathrm{g} / \mathrm{mol}]\end{array}$ & $\begin{array}{c}M W_{\mathrm{dif}} \\
\text { (DSE) [\%] }\end{array}$ & $\begin{array}{c}M W_{\mathrm{dif}} \\
(\mathrm{DSE})[\%]\end{array}$ & $\begin{array}{c}M W_{\mathrm{dif}} \\
\text { (DSE) [\%] }\end{array}$ \\
\hline CpLi & $225 \pm 17$ & $218 \pm 11$ & -1 & 32 & 98 \\
\hline $\mathrm{CpNa}$ & $310 \pm 25$ & $295 \pm 16$ & -21 & 3 & 57 \\
\hline CpK & $354 \pm 30$ & $335 \pm 18$ & -26 & -4 & 48 \\
\hline CpRb & $309 \pm 25$ & $294 \pm 16$ & $\mathbf{0}$ & 25 & 100 \\
\hline CpCs & $1864 \pm 198$ & $1624 \pm 111$ & - & - & - \\
\hline
\end{tabular}

The results fit best for monomeric aggregates with different quantities of coordinated THF per alkali metal (except for CpCs); hence, these are proposed to be the most populated species for CpMs in THF solution (see Figure 2-15). Just considering the $M W_{\text {det }}$ of dimeric aggregates, without or with a minimum of coordinated THF, they would also be an option, e.g. $M W_{\text {dif }}$ (DSE; $\left.\left[(\mathrm{CpLi})_{2}(\mathrm{THF})\right]\right)=-1 \%$ (the complete set of data for the other CpMs is given in the appendix). However, such dimeric aggregates are more unlikely. They do not only presume a very low coordination number, but, e.g. for $\left[(\mathrm{CpLi})_{2}(\mathrm{THF})\right]$ it is also difficult to imagine a bonding situation where only one lithium resonance would be observable at lower temperatures, even with fast exchange. Metallocene-type aggregates e.g. $\left[\mathrm{Cp}_{2} \mathrm{M}(\mathrm{THF})_{\mathrm{x}}\right]^{-}$cannot be differentiated from $\left[\mathrm{CpM}(\mathrm{THF})_{\mathrm{x}+1}\right]$, because $M W(\mathrm{THF}) \approx M W(\mathrm{Cp})$. Therefore, the question of existing metallocene-type species in solution cannot be irrevocably answered by the ECC technique. 


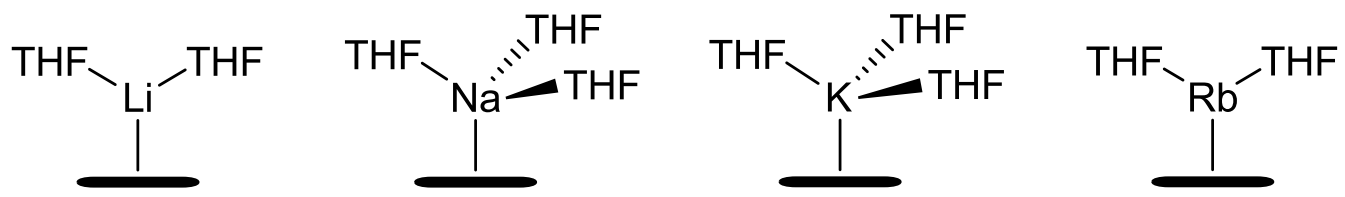

Figure 2-15: Proposed monomeric aggregates of $\mathrm{CpLi}, \mathrm{CpNa}, \mathrm{CpK}$ and $\mathrm{CpRb}$ in THF solution.

For CpLi the predominant monomeric aggregate seems to be coordinated by only two THF molecules. This molecule is stable over a large temperature range. If normalized $\log \left(D_{\mathrm{x}, \mathrm{norm}}\right)$ are compared, there is almost a perfect fit for all measured temperatures: $\log \left(D_{\mathrm{x}, \mathrm{norm}}\right)\left(\mathrm{CpLi}, 50^{\circ} \mathrm{C}\right)=$ $-8.886 ; \log \left(D_{\mathrm{x}, \text { norm }}\right)\left(\mathrm{CpLi}, 25^{\circ} \mathrm{C}\right)=-8.901 ; \log \left(D_{\mathrm{x}, \text { norm }}\right)\left(\mathrm{CpLi},-50^{\circ} \mathrm{C}\right)=-8.905 ; \log \left(D_{\mathrm{x}, \text { norm }}\right)(\mathrm{CpLi}$, $\left.-80^{\circ} \mathrm{C}\right)=-8.909 ; \Delta \log \left(D_{\mathrm{x}, \mathrm{norm}}\right)\left(\mathrm{CpLi}, 50 /-80^{\circ} \mathrm{C}\right)=0.023$. Furthermore, the formation of CIPs can be deduced from the identical $\log \left(D_{\mathrm{x}, \text { norm }}\right)$ values from the ${ }^{7} \mathrm{Li}$ - and ${ }^{1} \mathrm{H}-\mathrm{DOSY}$ NMR spectra (see Figure 2-16 and Table 5-26 in the appendix).

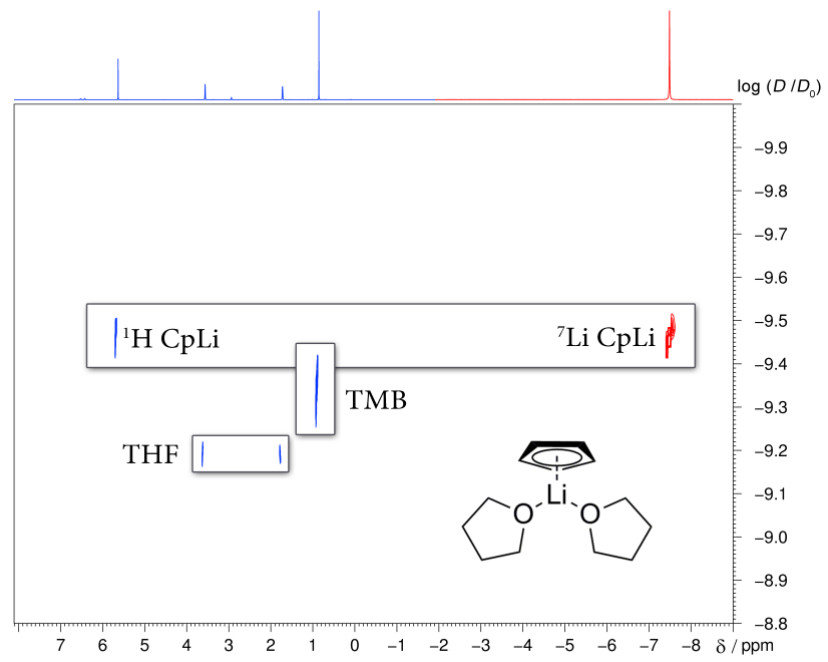

Figure 2-16: Superposition of ${ }^{1} \mathrm{H}$ - (blue) and ${ }^{7} \mathrm{Li}-\mathrm{DOSY}$ (red) NMR spectra of CpLi in THF- $d_{8}$ at $-50{ }^{\circ} \mathrm{C}$. Since only $15 \mathrm{~mm}$ solutions of CpLi were used, not all THF is coordinated to CpLi and the diffusion coefficients are averaged with free THF. $\left(D_{0}=1 \mathrm{~m}^{2} / \mathrm{s}\right)$

For $\mathrm{CpNa}$ and $\mathrm{CpK}$ the coordination by 3 THF molecules is preferred with a slightly bigger $M W_{\text {dif }}$ for $\mathrm{CpK}$. Cooling $\mathrm{CpNa}$ to $-50^{\circ} \mathrm{C}$ resulted in almost identical diffusion coefficients as were observed at $25^{\circ} \mathrm{C}\left(\log \left(D_{\mathrm{x}, \text { norm }}\right)\left(\mathrm{CpNa}, 25^{\circ} \mathrm{C}\right)=-8.978 ; \log \left(D_{\mathrm{x}, \text { norm }}\right)\left(\mathrm{CpNa},-50^{\circ} \mathrm{C}\right)=-8.971\right.$; $\Delta \log \left(D_{x, \text { norm }}\right)\left(\mathrm{CpNa}, 25 /-50^{\circ} \mathrm{C}\right)=0.007$. It was previously proposed that the mono-solvated $[\mathrm{CpNa}(\mathrm{THF})]$ is a possible aggregate in the solid state which could not be confirmed for solution. ${ }^{[59 \mathrm{~m}]}$ Cooling led to precipitation of $\mathrm{CpK}, \mathrm{CpRb}$ and $\mathrm{CpCs}$, therefore no further insight into thermal dependence could be gained for either of them.

Interestingly, the normalized diffusion coefficient of $\mathrm{CpRb}\left(\log \left(D_{\mathrm{x}, \mathrm{norm}}\right)\left(\mathrm{CpRb}, 25^{\circ} \mathrm{C}\right)=-8.9770\right)$ is elevated in comparison to $\mathrm{CpK}\left(\log \left(D_{\mathrm{x}, \mathrm{norm}}\right)\left(\mathrm{CpK}, 25^{\circ} \mathrm{C}\right)=-9.0098\right)$. Hence, $\mathrm{CpRb}$ seems to be coordinated by only 2 THF molecules at $25^{\circ} \mathrm{C}$. In literature, a coordination polymer of $\mathrm{CpRb}$ is known which crystallized with two THF molecules attached to the metal. ${ }^{[127]}$ This provides 
plausibility to the disaggregation in solution by breaking one of the two $\mathrm{Cp}-\mathrm{Rb}$ bonds. This atypical trimeric coordination of $\mathrm{Rb}$ in solution is supposedly due to the fading donor capacity of THF towards increasingly soft Lewis acidic cations. This can be underlined by alkali metal/ammonia binding energies $\left(\Delta E\left(\mathrm{M}^{+}-\mathrm{NH}_{3}\right)=170.3(\mathrm{Li}) ; 117.2(\mathrm{Na}) ; 82.0(\mathrm{~K}) ; 71.1(\mathrm{Rb}) ; 61.9(\mathrm{Cs}) \mathrm{kJmol}^{-1}\right) .^{[128]}$ These correspond with recently observed structures for hexamethyldisilazides crystallized from liquid ammonia. ${ }^{[15]}$ The same analogous trend can be assumed for $\Delta E\left(\mathrm{M}^{+}-\mathrm{THF}\right)$, as the heavier alkali metal $\mathrm{Cp}$ compounds tend to form coordination polymers instead of taking on more coordinated solvent molecules. $\mathrm{Rb}$ seems to be the borderline case of not adding solvent molecules and not coordinating additional cyclopentadienide ligands.

DOSY NMR spectroscopic measurements show that only CpCs forms oligomeric aggregates. As described above, such formations of coordination polymers are more prevalent among caesium than rubidium compounds. The observed aggregates display a $M W>1500 \mathrm{~g} / \mathrm{mol}$ $\left(\log \left(D_{x, \text { norm }}\right)\left(\mathrm{CpCs}, 25^{\circ} \mathrm{C}\right)=-9.407\right)$ and stay intact after heating to $50^{\circ} \mathrm{C}\left(\log \left(D_{\mathrm{x}, \mathrm{norm}}\right)\left(\mathrm{CpCs}, 50^{\circ} \mathrm{C}\right)\right.$ $\left.=-9.409, \Delta \log \left(D_{x, \text { norm }}\right)\left(\mathrm{CpCs}, 50 / 25^{\circ} \mathrm{C}\right)=0.002\right)$. Hence, it seems that very specific species are formed and retained. To gather further insights into this behavior of CpCs in THF, it was crystallized from a saturated THF solution. While this crystallization was effortlessly achieved at slightly lowered temperatures $\left(4^{\circ} \mathrm{C}\right.$ ) or even ambient temperature, the crystallized needles (see Figure 2-17) exhibited some limitations towards crystal structure analysis.

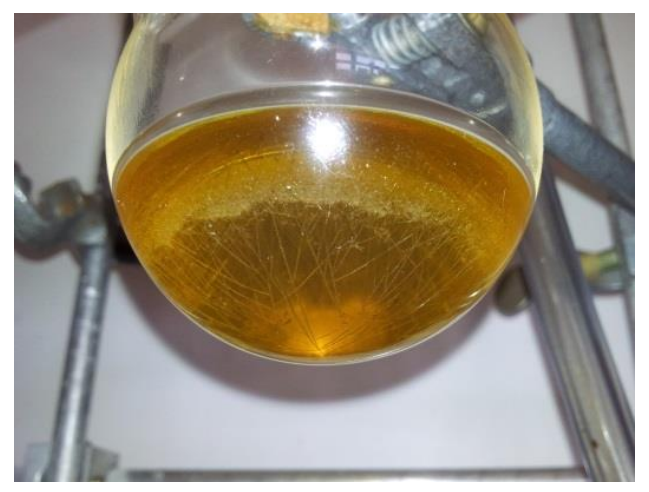

Figure 2-17: Needles of CpCs which were crystallized at ambient temperature in THF solution.

The crystals were sensitive towards air and moisture and furthermore displayed a phase transition upon cooling. After several attempts only a poor dataset could be acquired at $-23^{\circ} \mathrm{C}$. This data did not fulfill quality requirements for publication, since it displayed a modulated structure. Still, a polymeric setup with two coordinated THF molecules is indicated from the data which seems reasonable, as this has been previously observed for other related CpM structures. ${ }^{[59 k, 59 \mathrm{~m}, 127]}$ However, due to the low quality of the data, crystallographic parameters will not be discussed in this work.

In 1996, Harder and Prosenc could show the formation of a caesocene triple-decker. ${ }^{[69]}$ From this and the estimated $M W$, pentameric $\left[(\mathrm{CpCs})_{5}(\mathrm{THF})_{10}\right]\left(M W_{\text {dif }}(\right.$ merged $\left.)=-8 \%\right)$ or hexameric $\left[(\mathrm{CpCs})_{6}(\mathrm{THF})_{12}\right]\left(M W_{\text {dif }}(\right.$ merged $\left.)=12 \%\right)$ cyclic structures can be envisioned for the solution state 
structure of CpCs (see Figure 2-18), whereas other motifs (linear structures) with different amounts of THF or Cp are feasible, as well.
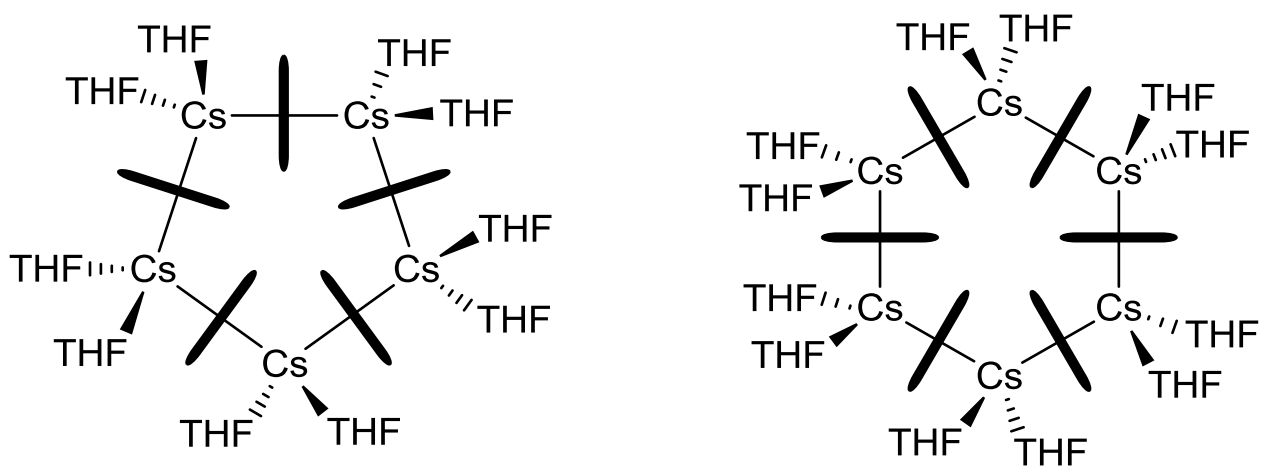

Figure 2-18: Proposed pentameric (left) and hexameric (right) cyclic aggregates of CpCs in THF solution.

The assumption of such cyclic structures can also be made, since high hapticities are known to prevail with soft Lewis acidic cations like $\mathrm{Cs}^{+} \cdot{ }^{[79 \mathrm{~b}, 129]}$ Also, similar hexameric structures have been observed in the solid state for bis(trimethylsilyl)cyclopentadienylthallium ${ }^{[130]}$ and pentamethylcyclopentadienylindium ${ }^{[131]}$. Still, it needs to be emphasized that current ECCs are not optimized for accurate $M W$ estimations of such aggregates, since reference compounds do not cover $M W s$ > $600 \mathrm{~g} / \mathrm{mol}$, yet.

\subsubsection{Solution State Structures of CpMs in ammonia and DMSO- $d_{6}$}

In the last section, solution state structures of CpMs in THF have been presented after utilizing ECC-MW estimations. Analyzing their aggregation in other solvents, for which different aggregational motifs could be observed in the solid state, are of similar interest, as they present new opportunities of fine-tuning reactivities. Structures which were crystallized from ammonia, i.e. $\left[(\mathrm{CpLi})_{2}\left(\mathrm{NH}_{3}\right)_{3}\right]$ and $\left[\mathrm{Li}\left(\mathrm{NH}_{3}\right)_{4}\right][\mathrm{Cp}]$, are especially fascinating, since they reveal on the one hand the formation of an SSIP containing naked $\mathrm{Cp}^{-}$and on the other a lithocene-type structure which may be preserved in solution as well. ${ }^{[290]}$ Furthermore, these crystal structures show that different aggregational states may be achieved depending on ammonia concentrations. ${ }^{[290]}$

It is reasonably difficult to control ammonia concentrations, since it is gaseous at ambient temperatures. Hence, the establishment of ECCs which are dependent on solvent viscosity would not be feasible. Still, CpLi was dissolved within pure ammonia (the procedure is given in section 4.2.1.1) in a sealed NMR tube with little amounts of toluene for referencing and measured at $25^{\circ} \mathrm{C}$. A chemical shift for the ${ }^{7} \mathrm{Li}$ NMR signal of $\delta=-0.59 \mathrm{ppm}$ was observed which is an immense difference compared to findings in THF $(\delta=-7.75 \mathrm{ppm})$, while the resonances in the proton and carbon spectra only varied slightly from THF measurements $\left({ }^{1} \mathrm{H}: \Delta \delta=0.2 \mathrm{ppm} ;{ }^{13} \mathrm{C}\right.$ : $\Delta \delta$ $=0.7 \mathrm{ppm})$. As was already stated, the strong high field shift of the ${ }^{7} \mathrm{Li}$ signal in THF is a consequence of the $\eta^{5}$-coordination of the alkali metal. Therefore, the difference in chemical shift 
observed in ammonia is a clear indication that this coordination behavior has changed. Additionally, a coupling between ammonia and lithium could be observed in a ${ }^{7} \mathrm{Li},{ }^{1} \mathrm{H}-\mathrm{HOESY}$ experiment, while a similar coupling to the cyclopentadienide could not be found (see Figure 2-19). All this points towards the formation of SSIPs. A ${ }^{1} \mathrm{H}-\mathrm{DOSY}$ spectrum was measured which did not provide any further insights (see Figure 5-9 in the appendix). ${ }^{\mathrm{a}}$

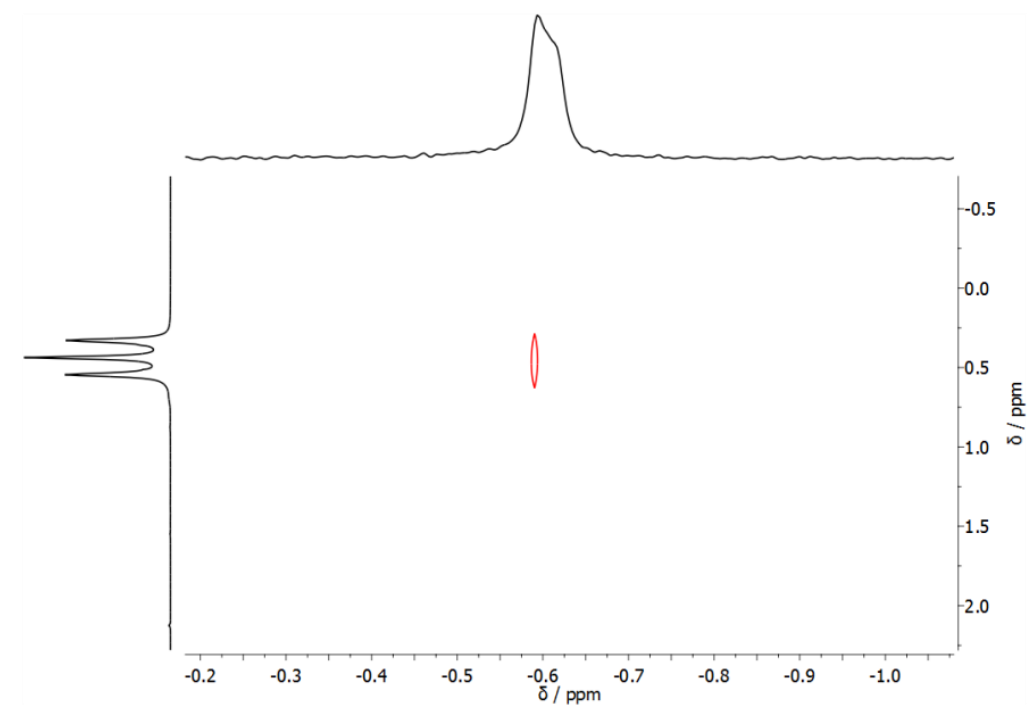

Figure 2-19: Excerpt from ${ }^{7} \mathrm{Li},{ }^{1} \mathrm{H}-\mathrm{HOESY}$ NMR spectrum of $\mathrm{CpLi}$ in ammonia at $25^{\circ} \mathrm{C}$. A coupling of ammonia and lithium is visible.

All CpMs were also fully characterized in DMSO- $d_{6}$. Due to its comparably high dielectric constant $(\varepsilon(D M S O)=47$; see Table 1-1 $)$ and the availability of ECCs, DMSO presents an opportunity for the observation of SSIPs that are comparable to species presumed for ammonia. Chemical shifts obtained from proton and carbon spectra in DMSO were similar for all CpM reagents $\left({ }^{1} \mathrm{H}: \delta=5.33\right.$ to $5.41 \mathrm{ppm},{ }^{13} \mathrm{C}: \delta=103.0$ to $\left.103.9 \mathrm{ppm}\right)$. These spectra also show only one nucleus for the CpMs. This indicates that no decomposition into e.g. starting material ${ }^{\mathrm{b}}$ took place, which might be possible if highly reactive SSIPs are formed. Chemical shifts observed for the alkali metals in DMSO were drastically shifted towards lower field compared to resonances observed in THF which is (same as before in ammonia) a good indication for an SSIP. The ${ }^{7} \mathrm{Li}$ resonance of CpLi $(\delta=$ $-1.07 \mathrm{ppm}$ ) is comparable to chemical shifts of solvated lithium-salts in DMSO shown in literature. ${ }^{[132]}$ The same is true for the ${ }^{23} \mathrm{Na}(\delta=-1$ to $-4 \mathrm{ppm})$ and ${ }^{133} \mathrm{Cs}$ resonances $(\delta=$ $-14.7 \mathrm{ppm}$ ) of $\mathrm{CpNa}$ and CpCs, respectively.

To further elucidate this behavior, DOSY and HOESY NMR experiments were employed: Similar to ammonia an NOE was observed between the solvent (DMSO) and lithium in a ${ }^{7} \mathrm{Li},{ }^{1} \mathrm{H}-\mathrm{HOESY}$ experiment of CpLi (see Figure 5-10 in the appendix), while no coupling to the cyclopentadienide was visible. The formation of SSIPs for DMSO can be further underlined by the differences of normalized diffusion coefficients received from ${ }^{1} \mathrm{H}$ - and ${ }^{7} \mathrm{Li}$-DOSY NMR (see Figure 2-20; the

\footnotetext{
${ }^{\text {a }} \mathrm{Li}$-DOSY was not measured.

${ }^{\mathrm{b}}$ Dicyclopentadiene has multiple resonances and was measured in DMSO- $d_{6}$ for comparison.
} 
complete set of data is given in the appendix: Table 5-27). Unfortunately, such a comparison was not possible for the other CpMs, since DOSY experiments of the other alkali metals could not be attained. Furthermore, HOESY spectra would be ineffective for the other alkali metals, as discussed in section 1.2.1.1. Still, these findings seem to confirm the formation of SSIPs at least for CpLi.

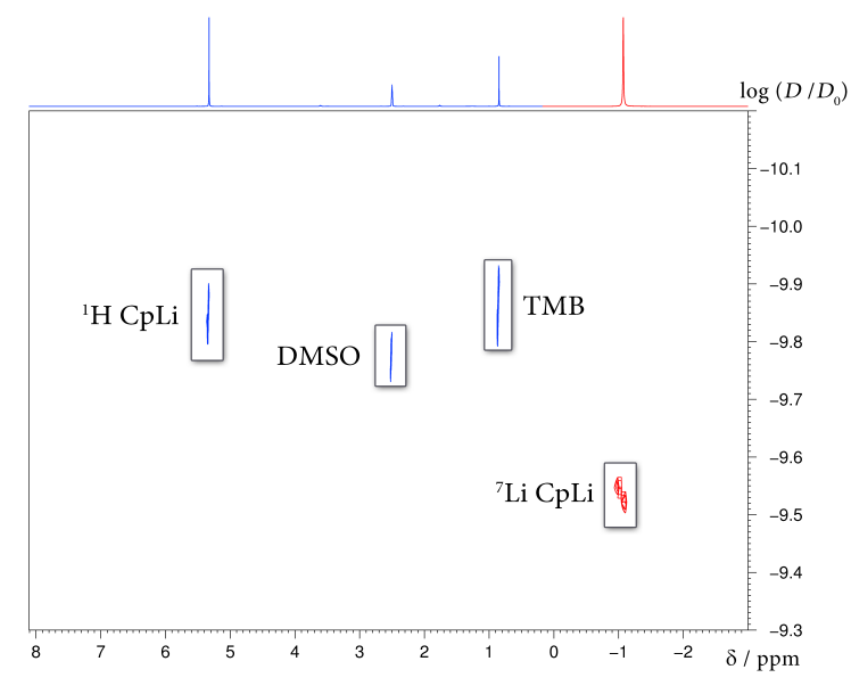

Figure 2-20: Superposition of ${ }^{1} \mathrm{H}$ - (blue) and ${ }^{7} \mathrm{Li}$-DOSY NMR spectra (red) of CpLi in DMSO- $d_{6}$ at $25^{\circ} \mathrm{C}$. This superposition shows a clear discrepancy of the diffusion coefficients of the cyclopentadienide and lithium ions as expected for the formation of SSIPs. $\left(D_{0}=1 \mathrm{~m}^{2} / \mathrm{s}\right)$

To transfer these indicative findings from CpLi to the other CpMs and further clarify the specific types of aggregates that are formed, normalized diffusion coefficients from ${ }^{1} \mathrm{H}$-DOSY can be compared amongst each other and ECC-MW predictions can be employed. For all CpMs (except CpCs) almost identical values for the normalized diffusion coefficients (see Table 2-10) were measured. Hence, it can be rationalized that SSIPs are formed by $\mathrm{CpNa}, \mathrm{CpK}$ and $\mathrm{CpRb}$ as well, since CIPs would result in differences of their diffusion coefficients of at least the contribution of the respective alkali metals and possibly differences in solvation.

Table 2-10: ECC ${ }^{\text {DMSO }}$ (DSE, ED and merged) were used to predict $M W_{\text {det }}$ of CpMs in DMSO- $d_{6}$. The accuracy of the $\mathrm{ECC}^{\mathrm{DMSO}}$ (DSE) is in the range of $M W_{\mathrm{dif}}= \pm 10 \%$, of the $\mathrm{ECC}^{\mathrm{DMSO}}(\mathrm{ED})$ is in the range of $M W_{\mathrm{dif}}= \pm 4 \%$ and of the ECC EMSO $^{\mathrm{D}}$ (merged) in the range of $M W_{\mathrm{dif}}= \pm 20 \%$. Theoretical errors as discussed in section 2.1.3 are given as standard deviations for all estimated $M W$ s. All results in this table are from measurements at $25^{\circ} \mathrm{C} . \log \left(D_{\mathrm{x}, \mathrm{norm}}\right)$ are also given for comparison.

\begin{tabular}{|c|c|c|c|c|}
\hline & $\log \left(D_{\mathrm{x}, \text { norm }}\right)$ & $\begin{array}{c}M W_{\text {det }} \\
\text { (merged) }[\mathrm{g} / \mathrm{mol}]\end{array}$ & $\begin{array}{c}M W_{\mathrm{det}} \\
(\mathrm{DSE})[\mathrm{g} / \mathrm{mol}]\end{array}$ & $\begin{array}{c}M W_{\mathrm{det}} \\
(\mathrm{ED})[\mathrm{g} / \mathrm{mol}]\end{array}$ \\
\hline $\mathrm{CpLi}$ & -9.2763 & $123 \pm 10$ & $129 \pm 8$ & $144 \pm 10$ \\
\hline $\mathrm{CpNa}$ & -9.2733 & $122 \pm 10$ & $128 \pm 6$ & $143 \pm 10$ \\
\hline CpK & -9.2706 & $121 \pm 9$ & $127 \pm 6$ & $142 \pm 10$ \\
\hline $\mathrm{CpRb}$ & -9.2671 & $119 \pm 9$ & $125 \pm 6$ & $141 \pm 10$ \\
\hline CpCs & -9.3699 & $172 \pm 15$ & $175 \pm 7$ & $186 \pm 15$ \\
\hline$\left({ }^{7} \mathbf{L i}\right) \mathrm{CpLi}$ & -8.9039 & $43 \pm 3$ & $49 \pm 5$ & $64 \pm 4$ \\
\hline
\end{tabular}

ECC- $M W$ predictions (see Table 2-10) show that the $M W$ s of all the CpMs (except CpCs) for the merged ECC are about $120 \mathrm{~g} / \mathrm{mol}$ (similar values are obtained for the DSE ECC; the complete set of 
data is given in the appendix: Table 5-27 (CpLi); Table 5-29 (CpNa); Table 5-31 (CpK); Table 5-33 (CpRb); Table 5-35(CpCs)). Since the formation of SSIP-type aggregates seems confirmed by the findings of CpLi stated above, the estimated $M W$ s pose the question of how exactly they aggregate. The estimated $M W$ s could be interpreted directly and viewed as unambiguous like it was done previously for CpMs in THF solution. However, for THF reasonable aggregates could be presented, whereas the estimated MWs for DMSO are not that conclusive. If the assumption of complete SSIP formation was true, only $\mathrm{Cp}^{-}$anions with a $M W$ of $65 \mathrm{~g} / \mathrm{mol}$ should be observed. This is obviously not the case, as the estimated $M W s$ are nearly doubled. ${ }^{a}$ While these $M W s$ would fit the starting material (dicyclopentadiene), decomposition can be disregarded, as well. Taking a step back from the SSIP assumption, the estimated MWs also exclude even most monomeric CIP structures with and without coordinated solvent, let alone dimeric or metallocene-like aggregates. ${ }^{\mathrm{b}}$

Hence, interpreting the predicted $M W$ s directly does not result in any reasonable aggregates. Some approximation within the methodology may prohibit a reasonable analysis: In section 2.1.4 the influence of elevated van-der-Waals densities was discussed which can be mostly disregarded here, since $\mathrm{Li}, \mathrm{Na}$ and $\mathrm{K}$ aggregates display normal $M D_{\mathrm{W}}$. Note that some $\mathrm{CIP}$ structures produce elevated $M D_{\mathrm{W}}$ values for $\mathrm{CpRb}$ and $\mathrm{CpCs}\left(M D_{\mathrm{w}}([\mathrm{CpRb}])=6.09 \cdot 10^{29} \mathrm{~g} /\left(\mathrm{mol} \cdot \mathrm{m}^{3}\right)\right.$; $M D_{\mathrm{W}}([\mathrm{CpRb}(\mathrm{DMSO})])=6.33 \cdot 10^{29} \mathrm{~g} /\left(\mathrm{mol} \cdot \mathrm{m}^{3}\right) ; \quad M D_{\mathrm{W}}([\mathrm{CpCs}])=6.60 \cdot 10^{29} \mathrm{~g} /\left(\mathrm{mol} \cdot \mathrm{m}^{3}\right) ;$ $\left.M D_{\mathrm{w}}([\mathrm{CpCs}(\mathrm{DMSO})])=6.68 \cdot 10^{29} \mathrm{~g} /\left(\mathrm{mol} \cdot \mathrm{m}^{3}\right)\right)$. Another important aspect is that the whole methodology is based on measured self-diffusion coefficients. Any influence on the self-diffusion of the particles is therefore also accumulated within the estimated MWs. In the introduction, it was stated that the diffusion coefficients can display averaged values for multiple aggregates in an equilibrium which might be the case for $\mathrm{CpMs}$ in DMSO. Hence, temperature-dependent measurements were performed exemplary for $\mathrm{CpNa}\left(25^{\circ} \mathrm{C}, 50^{\circ} \mathrm{C}\right.$ and $\left.75^{\circ} \mathrm{C}\right)$ which resulted in an increase of the diffusion coefficient $\left(\Delta \log \left(D_{x, \text { norm }}\right)\left(\mathrm{CpNa}, 75 / 25^{\circ} \mathrm{C}\right)=0.027\right)$ upon heating that exceeded significance (the complete set of data is given in the appendix: Table 5-29). This may be a hint for dynamic behavior, but unfortunately does not offer other new insights, as predicted $M W \mathrm{~s}$ are still within a similar margin.

Other intermolecular effects can also affect self-diffusion: For ammonia, the comparison to the $\left[\mathrm{Li}\left(\mathrm{NH}_{3}\right)_{4}\right][\mathrm{Cp}]$ structure was drawn. This solid state structure indicates the formation of a "two-dimensional net with tailored meshes bridged by hydrogen bonding to free lattice ammonia"[290], thus forming channels, wherein the anionic cyclopentadienides are shielded by solvent molecules from the solvent-coordinated lithium cations (see Figure 2-21).

While the formation of such polar channels is questionable in an isotropic solution and no direct comparison to a crystal structure in DMSO can be made, since all attempts to crystallize CpMs

\footnotetext{
${ }^{\text {a }}$ Note that estimated $M W \mathrm{~s}$ fit for the ED-ECC $[\mathrm{Cp}(\mathrm{DMSO})]^{-}\left(M W_{\text {calc }}=143 \mathrm{~g} / \mathrm{mol}\right)$.

${ }^{\mathrm{b}}$ They fit incidentally for $[\mathrm{CpK}]$ and $[\mathrm{CpCs}]$ which are deemed unlikely in the strongly coordinating solvent DMSO.
} 


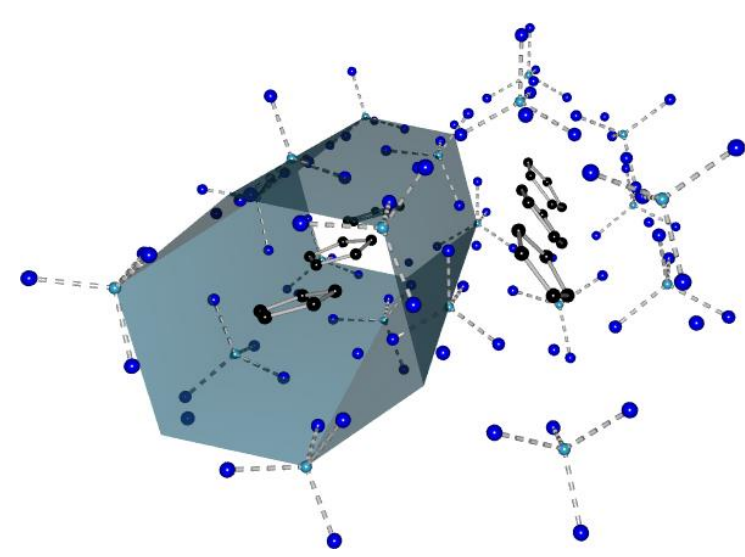

Figure 2-21: Polar channels for the accommodation of cyclopentadienide anions shown in the crystal structure of $\left[\mathrm{Li}\left(\mathrm{NH}_{3}\right)_{4}\right][\mathrm{Cp}]$. Reprinted from reference [29o]. Copyright $\odot 2010$ American Chemical Society.

from DMSO failed, a the overall similar behavior to ammonia may hint to some (partial) association or formation of coordinative bonds as the reason for the observed elevated MWs of the cyclopentadienide. In future, conductivity or further temperature-dependent measurements of other CpMs might lead to a more conclusive explanation. However, in this case, the ECC methodology alone does not give clear results about the aggregation of CpMs. This nicely illustrates the methodology's current limits, as diffusion-influencing effects that are not cancelled due to referencing have to be considered and known a priori for a successful interpretation.

Lastly, CpCs will be discussed shortly, since it was the only CpM that displayed a significantly different diffusion coefficient in DMSO: Its estimated $M W$ was about $170 \mathrm{~g} / \mathrm{mol}$. While, similar to $\mathrm{CpLi}$ and $\mathrm{CpNa}$, the alkali metal shift of $\mathrm{CpCs}\left(\delta\left({ }^{133} \mathrm{Cs}\right)=-14.7 \mathrm{ppm}\right)$ was shifted substantially towards lower field compared to findings in THF $\left(\delta\left({ }^{133} \mathrm{Cs}\right)=-240.1 \mathrm{ppm}\right)$, it is still significantly different to shifts observed for completely solvent-separated $\mathrm{Cs}^{+}$cations (caesium-salts) in DMSO presented in literature by DeWitte et al. $(\delta=68 \mathrm{ppm}) \cdot{ }^{[133]}$ DeWitte et al. also mention that $\mathrm{Cs}^{+}$ions are more likely to form CIPs than the other alkali metals. ${ }^{[133]}$ This is also be indicated by the findings herein. The possible equilibrium, indicated by the temperature-dependent measurements of $\mathrm{CpNa}$, might be pushed towards the CIP side for CpCs. Unfortunately, this behaviour cannot be further investigated, as cooling is not possible for DMSO

\subsubsection{Solution State Structures of Methylated Alkali Metal Cyclopentadienides in THF- $d_{8}$}

To further augment the acquired knowledge on aggregation and solvation as well as obtain additional information on the effects of methyl substituents on CpM aggregation, synthetically

\footnotetext{
${ }^{a}$ Even attempts to diffuse nonpolar solvents (e.g. pentane) slowly into DMSO did not result in crystallization. After a few days the solution always turned dark in an argon glove box, indicating decomposition.
} 
accessible ${ }^{\mathrm{a}}$ mono- $(\mathrm{MeCpM})$ and pentamethylated $\left(\mathrm{Cp}^{*} \mathrm{M}\right)$ alkali metal cyclopentadienide reagents were analyzed by NMR (MeCpLi, Cp ${ }^{\star} \mathrm{Li}, \mathrm{Cp}^{\star} \mathrm{K}$ and $\mathrm{Cp}^{\star} \mathrm{Cs}$ ). The synthetic routes for these reagents were either already known in literature or simple one-step syntheses and will therefore not be discussed in the main part of this thesis (for further information see sections 4.2.2 and 4.2.3).

\subsubsection{Solution State Structure of MeCpLi in THF- $d_{8}$}

Neither in the solid state nor in solution has methylcyclopentadienyllithium (MeCpLi) been investigated intensely. Still, a single crystal structure of monomeric MeCpLi has been published by Hammel et al. in 1990 ([MeCpLi(TMEDA)]). ${ }^{[50 b]}$ Since the solution state structures of THF aggregates could be successfully investigated for underivatized CpMs with ECC-DOSY-MW predictions, this should be repeated for $\mathrm{MeCpLi}$ and the pentamethylated compounds in the following section. MeCpLi was synthesized in good yields (90\%) and was readily available for NMR spectroscopic analysis. Its ${ }^{1} \mathrm{H}$ and ${ }^{13} \mathrm{C}$ NMR spectra as well as the ${ }^{7} \mathrm{Li}$ resonance which was observed at $-7.6 \mathrm{ppm}$ indicate a single aggregate at $25^{\circ} \mathrm{C}$ (see section 4.2.2). While multiplicities are comparable, the ${ }^{1} \mathrm{H}$ chemical shifts are a little different compared to literature. ${ }^{[134]}$

ECC-DOSY NMR spectroscopy was also employed to investigate MeCpLi. The estimated $M W_{\text {det }}(\mathrm{DSE})$ of $237 \mathrm{~g} / \mathrm{mol}$ was compared to $M W_{\text {calc }}$ of likely monomeric [MeCpLi(THF) $)_{\mathrm{x}}$ ] and dimeric aggregates $\left[(\mathrm{MeCpLi})_{2}(\mathrm{THF})_{\mathrm{x}}\right]$ with $\mathrm{x}=0-4$ (the complete set of data is given in the appendix: Table 5-36). This comparison indicates the clear preference of aggregation as $\left[\mathrm{MeCpLi}(\mathrm{THF})_{2}\right]\left(M W_{\text {dif }}(\mathrm{DSE})=-3 \%\right)$ which is isostructural to species found for CpLi, i.e. $\left[\mathrm{CpLi}(\mathrm{THF})_{2}\right]$. Interestingly, these solution state structures underline the preference of the coordination number three for such lithium aggregates.

\subsubsection{Solution State Structures of $C p^{\star} \mathrm{Ms}$ in THF- $d_{8}$}

The pentamethylated alkali metal cyclopentadienides $\left(\mathrm{Cp}^{\star} \mathrm{Ms}\right)$ have been more thoroughly investigated in the solid state than the monomethylated MeCpMs: Crystal structures are known that range from the polymeric form without coordinating solvent to monomeric aggregates featuring donating Lewis bases such as pyridine and crown ethers. ${ }^{[56,56,566,59,59 h, 59 i]}$ These structures closely resemble the solid state structures of underivatized $\mathrm{CpM}$ reagents (see introduction section 1.1.2.4). Pentamethylcyclopentadienyllithium $\left(\mathrm{Cp}^{\star} \mathrm{Li}\right)$, -potassium $\left(\mathrm{Cp}^{\star} \mathrm{K}\right)$ and -caesium $\left(\mathrm{Cp}{ }^{\star} \mathrm{Cs}\right)$ were analyzed in this work. While $\mathrm{Cp}^{\star} \mathrm{K}$ and $\mathrm{Cp}^{\star} \mathrm{Cs}$ could be investigated straightforward

\footnotetext{
${ }^{a}$ Freshly cut, not further activated, elemental sodium was not reactive enough to metalate $\mathrm{MeCp}$ or $\mathrm{Cp}^{\star}$ (with otherwise the same reaction conditions as listed for MeCpLi in section 4.2.2). For further studies other sodium reagents (e.g. $\mathrm{NaH}$ or NaHMDS) should be utilized. Syntheses of the higher homologues of $\mathrm{MeCpNa}$ as well as of $\mathrm{Cp}^{\star} \mathrm{K}$ were not pursued.
} 
in THF solution, alas, $\mathrm{Cp}^{\star} \mathrm{Li}$ was not soluble. Hence, chemical shifts, normalized diffusion coefficients and estimated $M W$ s are only given for $\mathrm{Cp}^{\star} \mathrm{K}$ and $\mathrm{Cp}^{\star} \mathrm{Cs}$ in Table 2-11.

Table 2-11: Chemical shifts, normalized diffusion coefficients $\left(\log \left(D_{\mathrm{x}, \text { norm }}\right)\right)$ and estimated $M W_{\mathrm{det}}$ of $\mathrm{Cp}^{\star} \mathrm{K}$ and $\mathrm{Cp}^{\star} \mathrm{Cs}$. $\mathrm{ECC}^{\mathrm{THF}}$ (DSE and merged) were used to predict $M W_{\text {det }}$ in THF- $d_{8}$. The accuracy of the ECC ${ }^{\mathrm{THF}}$ (DSE) is in the range of $M W_{\text {dif }}= \pm 8 \%$ and of the ECC ${ }^{\mathrm{THF}}$ (merged) in the range of $M W_{\mathrm{dif}}= \pm 18 \%$. Theoretical errors as discussed in section 2.1.3 are given as standard deviations for all estimated $M W$ s. All results in this table are from measurements at $25^{\circ} \mathrm{C}$.

\begin{tabular}{|c|c|c|c|c|c|}
\hline & $\begin{array}{c}{ }^{1} \mathbf{H} \\
\delta[\mathrm{ppm}]\end{array}$ & $\begin{array}{l}{ }^{{ }^{13} \mathrm{C}\left\{{ }^{1} \mathrm{H}\right\}} \\
\delta[\mathrm{ppm}]\end{array}$ & $\log \left(D_{\mathrm{x}, \mathrm{norm}}\right)$ & $\begin{array}{c}M W_{\text {det }} \\
\text { (merged) }[\mathrm{g} / \mathrm{mol}]\end{array}$ & $\begin{array}{c}M W_{\mathrm{det}} \\
(\mathrm{DSE})[\mathrm{g} / \mathrm{mol}]\end{array}$ \\
\hline$C p^{*} K$ & $1.95(\mathrm{~s})$ & $104.8,11.4$ & -9.0393 & $401 \pm 34$ & $377 \pm 21$ \\
\hline $\mathrm{Cp}^{\star} \mathrm{Cs}$ & $1.85(\mathrm{~s})$ & $108.0,11.1$ & -9.1411 & $612 \pm 56$ & $564 \pm 33$ \\
\hline
\end{tabular}

For both compounds, only a single signal set was observable (see Figure 2-22 exemplary for $\mathrm{Cp}^{\star} \mathrm{K}$ ).

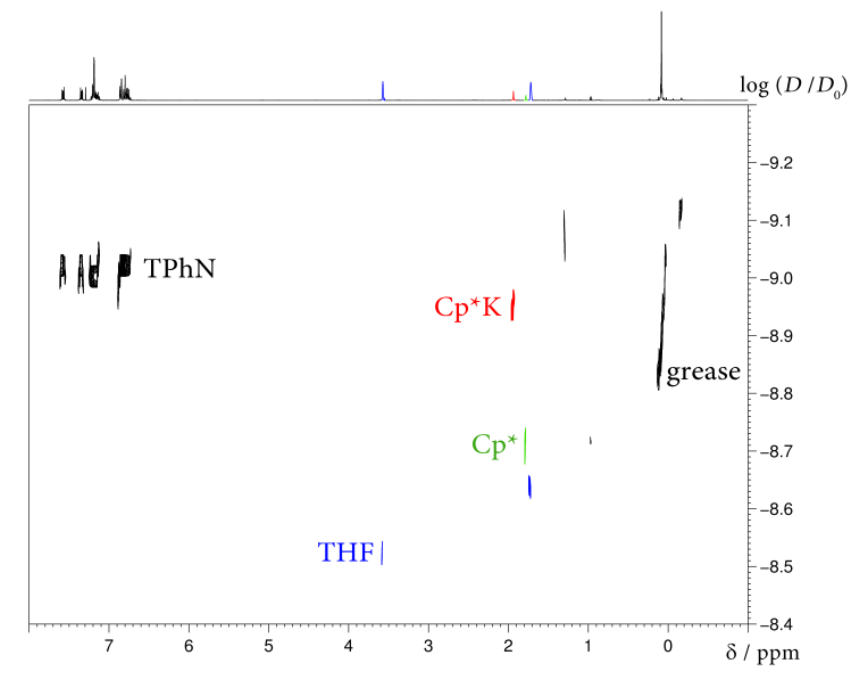

Figure 2-22: ${ }^{1} \mathrm{H}$-DOSY spectrum of $\mathrm{Cp}^{\star} \mathrm{K}$ in THF- $d_{8}$ at $25^{\circ} \mathrm{C}$. Since only $15 \mathrm{~mm}$ solutions of $\mathrm{Cp}^{\star} \mathrm{K}$ were used, not all THF is coordinated to $C \mathrm{p}^{\star} \mathrm{K}$ and the diffusion coefficients are averaged with free THF. Residues of the starting material (pentamethylcyclopentadiene; $\left.C \mathrm{p}^{*}\right)$ are also visible. $\left(D_{0}=1 \mathrm{~m}^{2} / \mathrm{s}\right)$

Since these compounds behaved like underivatized CpMs in the solid state, the same was anticipated for solution: Indeed, $\mathrm{Cp}^{\star} \mathrm{K}$ displayed the same aggregation and solvation $\left(\left[\mathrm{Cp}^{\star} \mathrm{K}(\mathrm{THF})_{3}\right] ; M W_{\mathrm{dif}}(\mathrm{DSE})=4 \%\right.$; the complete set of data is given in the appendix: Table 5-37) as $\mathrm{CpK}\left(\left[\mathrm{CpK}(\mathrm{THF})_{3}\right]\right)$. However, $\mathrm{Cp}^{\star} \mathrm{Cs}$ did not reveal oligomeric aggregation, as previously observed for CpCs, but instead seems to be aggregated in a monomeric fashion as $\left[\mathrm{Cp}^{*} \mathrm{Cs}(\mathrm{THF})_{4}\right]$ $\left(\left(M W_{\text {dif }}(\mathrm{DSE})=-1 \%\right.\right.$; the complete set of data is given in the appendix: Table $\left.5-38\right)$. The $\mathrm{Cp}^{\star} \mathrm{Cs}$ solution state structure indicates that the methyl groups prevent or hinder oligomeric aggregate formation, perhaps by introducing more steric demand. The increased coordination number of Cs that would be required for the predicted $M W$ might be explained by the inclination of Cs to form higher coordination polymers, as was already explained for the CpCs compound.

These two examples show that further investigations are promising. Possibly, other solvents can be utilized to solvate $\mathrm{Cp}^{*} \mathrm{Li}$. Moreover, temperature-dependent measurements might shed further light onto the aggregational behavior of all $\mathrm{Cp}^{\star} \mathrm{Ms}$ as well as investigations through alkali metal 
NMR. Additionally, the synthetic endeavors towards $\mathrm{MeCpMs}$ and $\mathrm{Cp}^{\star} \mathrm{Ms}$ should be continued to compliment this work.

\subsection{Combined Studies Involving Diffusion NMR to Solve Structural Ambiguities}

In the previous chapter, the ECC methodology was utilized to determine the aggregation and solvation of CpMs and some of their methylated derivatives in different deuterated solvents. While these findings enable a closer look into s-block chemistry of Group 1, other organometallic reagents have so far not been discussed. However, with the acquired knowledge of the ECC-DOSY methodology, the transferability towards Group 2 or even transition metal organometallics is possible and has already been demonstrated in several studies. ${ }^{[111-112]}$ Hence, in this chapter the ECC methodology and DOSY itself will be utilized to investigate reagents which were provided by collaborators. Each section will contain a different research area which will be introduced shortly, while the chapter will mainly concern insights acquired through NMR.

\subsubsection{Solution State Structures of Alkyl Grignard Reagents in THF- $d_{8}{ }^{a}$}

Prominent examples of Group 2 reagents are organomagnesium halides (RMgX; $\mathrm{R}=$ alkyl- or arylgroups; $\mathrm{X}=$ halides) which were first discovered in the year of 1900 by Grignard and are named accordingly Grignard reagents. ${ }^{[135]}$ Their applicability towards a multitude of reactants and the ability of carbon-carbon bond formation is widely utilized and has been reviewed and featured in literature extensively. ${ }^{[136]}$ Their use can be highlighted e.g. in the total synthesis of the natural antibiotic vancomycin. ${ }^{[137]}$

Accordingly, structure elucidation of these strong nucleophiles has been of interest for a long time. ${ }^{[138]}$ They were investigated early on by Schlenk and Schlenk, who showed that they undergo a disproportionation into dialkyl- or diaryl-magnesium compounds $\left(\mathrm{R}_{2} \mathrm{Mg}\right)$ and magnesium halides $\left(\mathrm{MgX}_{2}\right) .{ }^{[139]}$ Their complex solution behavior can be depicted with the so-called Schlenk equilibrium (see Scheme 2-2) which has since been refined by further studies, e.g. by Ashby et al. through NMRand IR-spectroscopic investigations. ${ }^{[140]}$

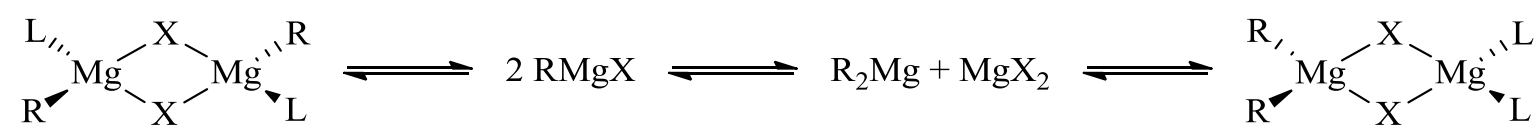

Scheme 2-2: Schlenk equilibrium of Grignard reagents, depicting the disproportionation and dimerization in solution. (L is a Lewis base or donating solvent, whereas $\mathrm{R}$ is an organic residue and $\mathrm{X}$ a halide)

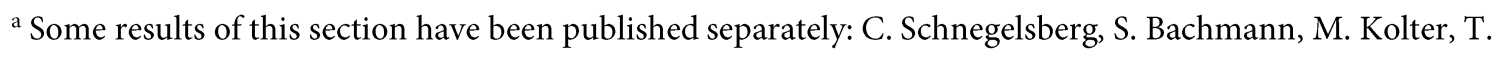
Auth, M. John, D. Stalke, K. Koszinowski, Chem. Eur. J. 2016, 22, 7752-7762.[3] 
While this equilibrium is very fast and no signal separation can be observed at room temperature on the NMR timescale, it could still be shown that Grignard reagents are prone to form either monomeric or dimeric species. Their formation is highly dependent on the solvent, the analyte's concentration, temperature and the reagents themselves. While in $\mathrm{Et}_{2} \mathrm{O}$ monomeric and dimeric species were observed simultaneously, in THF only monomeric species were found. ${ }^{[140 f]}$ However, the disproportionation is more pronounced in THF leading to increased amounts of $\mathrm{R}_{2} \mathrm{Mg}$ and $\mathrm{MgX}_{2}$.

In the solid state, Grignard reagents crystallized from bulk $\mathrm{RMgCl}$ solutions $(\mathrm{R}=\mathrm{Me}, t \mathrm{Bu}, \mathrm{Ph}, \mathrm{Bn})$ prefer forming a tetrameric open-cube that is depicted in Scheme 2-3..$^{[141]}$

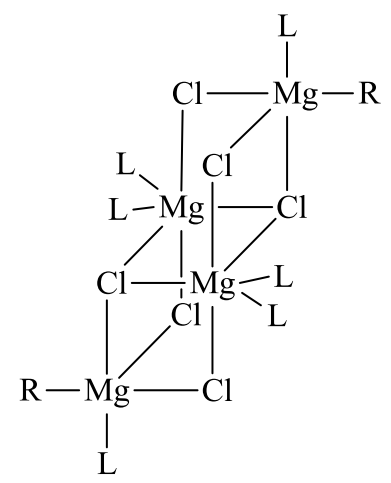

Scheme 2-3: Observed solid state structure after crystallization of bulk Grignard reagents displaying an open-cube setup. This specific open-cube was crystallized from a THF solution of $[i \mathrm{PrMgCl} \cdot \mathrm{LiCl}]$ layered with $\mathrm{Et}_{2} \mathrm{O}{ }^{[141 \mathrm{bl}]}$

Herein, conventional alkyl $\mathrm{RMgX}(\mathrm{R}=\mathrm{Et}, n \mathrm{Bu}, n \mathrm{Hex}, n \mathrm{Oct}, n \mathrm{Dec}, i \mathrm{Pr} ; \mathrm{X}=\mathrm{Cl}, \mathrm{Br})$ were investigated to elucidate their behavior in cooperation with the Koszinowski group. This study entailed a combination of electrospray-ionization (ESI) mass spectrometry (MS), electrical conductivity measurements, NMR spectroscopy and quantum chemical calculations. In this study, negative ion mode ESI mass spectrometry measurements of the different alkyl Grignard reagents in THF resulted in mainly trinuclear anionic species of the type $\left[\mathrm{R}_{n} \mathrm{Mg}_{3} \mathrm{Cl}_{7-\mathrm{n}}\right]^{-}$. An exemplary spectrum for $n \mathrm{BuMgCl}$ is shown in Figure 2-23. Those results stand in contrast to the classical Schlenk equilibrium and are most probably due to the increased analyte concentration in the charged droplets that are produced during the ESI process. The high selectivity for trinuclear anions is rooted in their close resemblance to the tetrameric open-cube solid state structures which could be further underlined by theoretical calculations. The subtraction of only a single $[\mathrm{MgX}]^{+}$ moiety leads to the corresponding trinuclear anion. ${ }^{[3]}$

To further compliment the ESI-MS findings, NMR spectroscopy was used. Notably, magnesium amide bases or so-called Hauser-bases $\left(\mathrm{R}_{2} \mathrm{NMgX}\right)$ which are closely related to Grignard reagents, have already been investigated via ECC-DOSY in our group. ${ }^{[111]}$ Hence, the solution state structures of alkyl Grignard reagents were investigated by the same technique in THF- $d_{8}$. For this study, $25 \mathrm{mM}$ solutions of $\mathrm{RMgX}$ with equimolar amounts of the internal reference 1,2,3,4-tetraphenylnaphthalene $(\mathrm{TPhN})$ were prepared. 


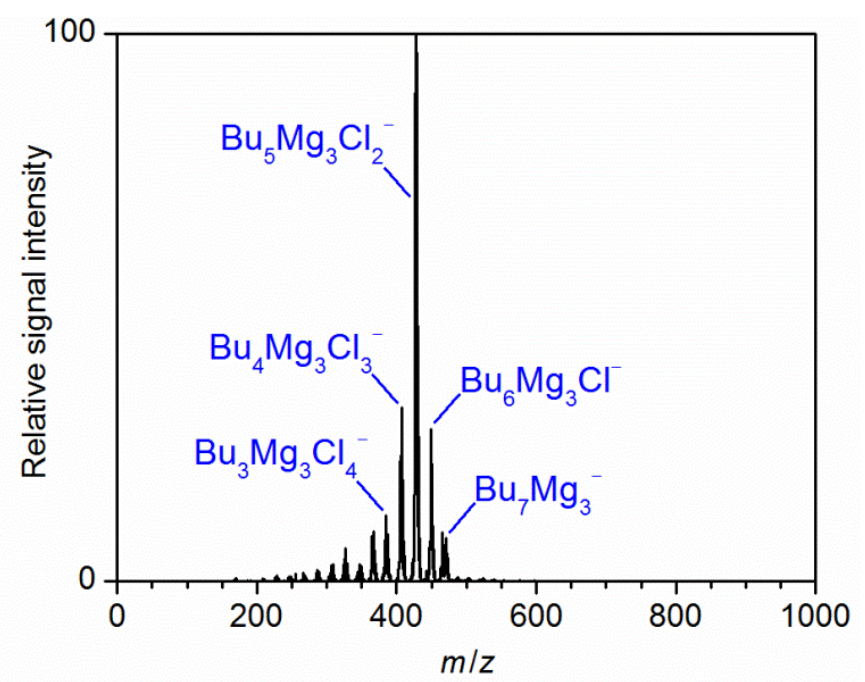

Figure 2-23: Negative ion-mode ESI mass spectrum of a $25 \mathrm{mM}$ solution of $n \mathrm{BuMgCl}$ in THF. ${ }^{[3]}$

The proton and carbon spectra of the alkyl Grignard systems showed the expected upfield-shifted signals of the methylene or methine groups at $25^{\circ} \mathrm{C}$, respectively, which were directly bound to the

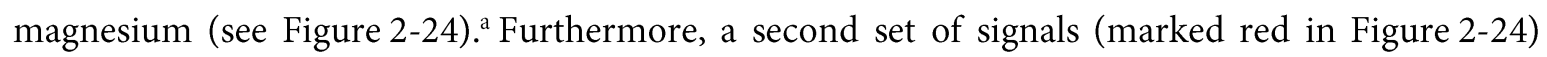
revealed the presence of minimum amounts of possibly starting material $\mathrm{RCl}$ for all systems. Cooling to $-50^{\circ} \mathrm{C}$ did not result in additional signals.

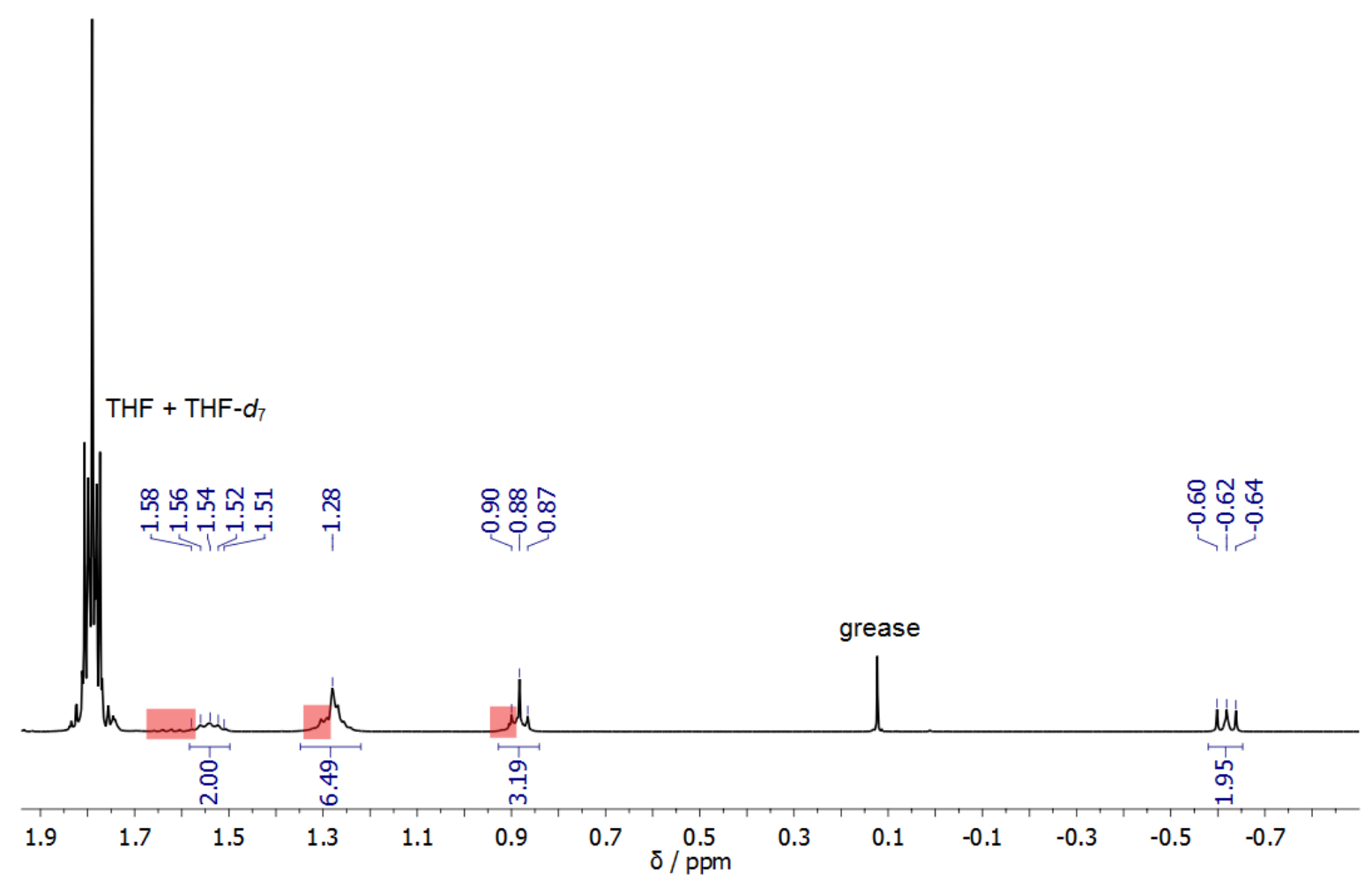

Figure 2-24: Excerpt from the ${ }^{1} \mathrm{H}$ NMR spectrum of $n \mathrm{HexMgCl}$ in THF- $d_{8}$ at $25^{\circ} \mathrm{C}$ reveals a second set of signals (marked in red).

${ }^{\text {a }}$ The complete set of 1D and DOSY NMR spectra is given in the Supporting Information of reference [3]. 
Additionally, ${ }^{1} \mathrm{H}$-DOSY NMR was used to determine the aggregation of Grignard reagents. In the DOSY spectra only their diffusion coefficients were observable, whereas the signal intensity of RCl was too low to gather any related information. An exemplary ${ }^{1} \mathrm{H}$-DOSY spectrum is shown in Figure 2-25.

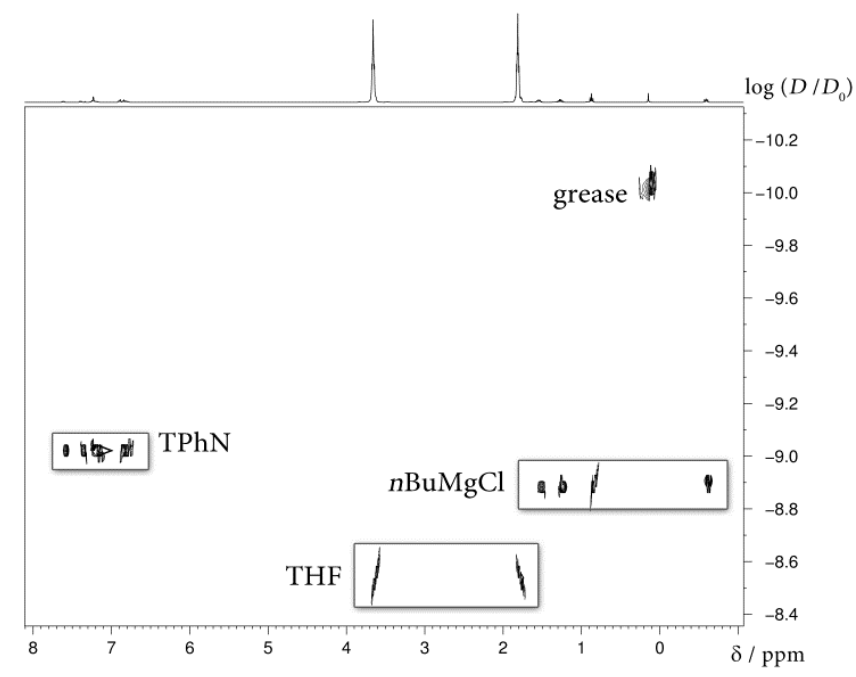

Figure 2-25: ${ }^{1} \mathrm{H}$-DOSY spectrum of $n \mathrm{BuMgCl}$ with the internal reference TPhN in THF- $d_{8}$ at $25^{\circ} \mathrm{C} .\left(D_{0}=1 \mathrm{~m}^{2} / \mathrm{s}\right)$

\subsubsection{External Calibration Curves for Rod-Like (RL) Molecules}

While the calibration curves for dissipated spheres and ellipsoids (DSE) have been proven to be effective for the $M W$ prediction of organometallic species before, in the case of the larger alkyl Grignard systems they would most certainly fail. ${ }^{\text {a }}$ The shapes of these reagents are dominated by the increasing lengths of the alkyl chains and cannot be described as spherical. Hence, references were sought that demonstrated a similar geometry to establish more specialized ECCs. References were mostly starting materials used in synthesis (e.g. 1-chlorohexane $\left(n \mathrm{HexCl} ; \mathrm{CH}_{3}\left(\mathrm{CH}_{2}\right)_{4} \mathrm{CH}_{2} \mathrm{Cl}\right)$ ) as well as other compounds incorporating long alkyl chains (e.g. batylalcohol (Batyl; $\mathrm{CH}_{3}\left(\mathrm{CH}_{2}\right)_{17-}$ $\left.\mathrm{OCH}_{2} \mathrm{CH}(\mathrm{OH}) \mathrm{CH}_{2} \mathrm{OH}\right)$ ). The comparability and suitability of these references can be easily confirmed by the calculation of principal moments of inertia $I$ and subsequently relative shape anisotropies $\kappa^{2}$, as explained in section 2.1.2. While it was demonstrated that the calculation of these principal moments of inertia would overall not lead to a more straightforward molecule categorization, they can still be an adequate indicator to distinguish linear from more spherical molecules. Model compounds that were intended to represent rod-like (RL) molecules incorporating alkyl chains all display shape anisotropies of more than $20 \%$ which is considerably higher than values observed for other model compounds (see Table 2-12). ${ }^{\mathrm{b}}$

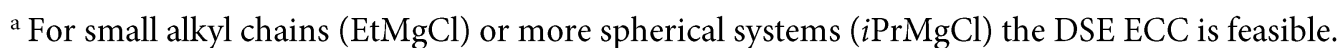

b Tetrabutylammoniumchloride (TBACl) was removed as model compound from the ECC for rod-like (RL) molecules, as it is more spherical than linear. Results based on the ECCs may differ slightly from literature. ${ }^{[3]}$
} 
Accordingly, utilizing the references listed in Table 2-12, an ECC for rod-like (RL) molecules, i.e. the alkyl Grignard systems, was established in THF- $d_{8}$.

Table 2-12: Principal moments of inertia calculated with PMIFST ${ }^{[122]}$ for geometry optimized (MMFF94) structures of model compounds used in ECCs for rod-like aggregates. The relative shape anisotropy $\kappa^{2}$ is also listed for each model compound.

\begin{tabular}{lccccc}
\hline Compound & $\boldsymbol{I}_{\mathbf{x x}}\left[\mathbf{u} \AA^{2}\right]$ & $\boldsymbol{I}_{\mathbf{y y}}\left[\mathbf{u} \AA^{2}\right]$ & $\boldsymbol{I}_{\mathbf{z z}}\left[\mathbf{u} \AA^{2}\right]$ & $\boldsymbol{I}_{\mathbf{x x}}: \boldsymbol{I}_{\mathbf{y y}}: \boldsymbol{I}_{\mathrm{zz}}$ & $\boldsymbol{\kappa}^{\mathbf{2}} \cdot \mathbf{1 0 0} \%$ \\
\hline Batylalcohol & 274 & 20730 & 20911 & $1: 76: 76$ & 24.0 \\
$\boldsymbol{n}$ DecCl & 70 & 3349 & 3387 & $1: 48: 48$ & 23.5 \\
$\boldsymbol{n} \mathbf{H e x C l}$ & 44 & 956 & 980 & $1: 22: 22$ & 21.8 \\
$\boldsymbol{n}$ OctCl & 57 & 1916 & 1948 & $1: 34: 34$ & 22.9 \\
\hline
\end{tabular}

Since also brominated reagents with elevated $M D_{\mathrm{W}}$ (see section 2.1.4), i.e. $n \mathrm{BuMgBr}$, were to be analyzed, another specialized ECC for rod-like molecules was established utilizing brominated references $^{\mathrm{a}}(\mathrm{RLBr}$; the complete set of normalized diffusion coefficients for RL and RLBr references is given in the appendix: Table 5-21). Note that only mono-brominated references can be described by the RLBr ECC. Figure 2-26 depicts these ECCs, whereas in Table 2-13 the corresponding ECC parameters as well as other quality factors, i.e. av. and max. deviations of back-calculated $M W$ s and cor. $\mathrm{R}^{2}$ values, are listed.
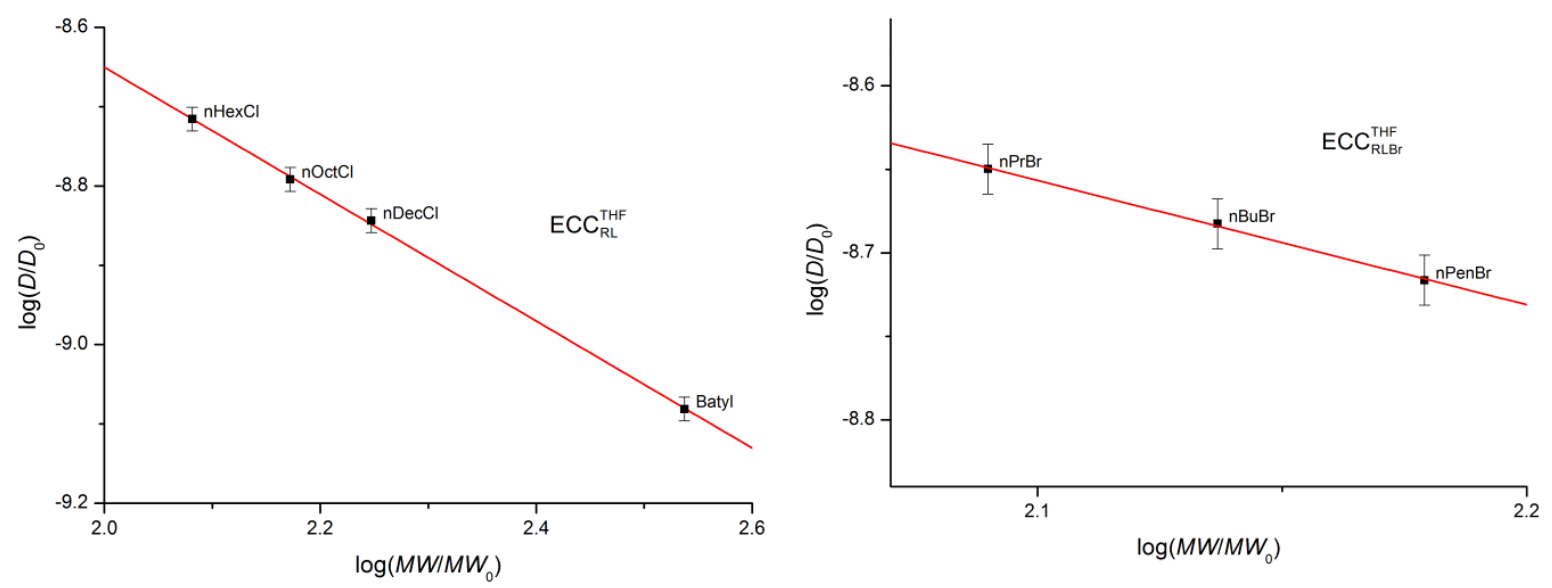

Figure 2-26: ECCs for rod-like (RL) (left) and brominated rod-like (RLBr) (right) compounds for THF- $d_{8} .\left(D_{0}=1 \mathrm{~m}^{2} / \mathrm{s}\right.$; $\left.M W_{0}=1 \mathrm{~g} / \mathrm{mol}\right)$

The same information is given for specialized ECCs developed in the master thesis of Kreyenschmidt for mono- $(\mathrm{Br})$ and di-brominated $\left(\mathrm{Br}_{2}\right)$ reagents. ${ }^{[124], \mathrm{b}}$

Since the reference count was quite limited for the RL and RLBr ECCs, theoretical errors are more feasible than empirical deviations. Theoretical errors are listed for the individual model compounds in Table 2-14. These were calculated according to the formulae described in section 2.1.3.

a Principal moments of inertia were not calculated for brominated references.

${ }^{\mathrm{b}}$ Molecules of the $\mathrm{Br}$ and $\mathrm{Br}_{2}$ ECCs are not differentiated by shape. 
Table 2-13: ECC parameters and their errors, av. and max. deviations of back-calculated $M W$ s and cor. $\mathrm{R}^{2}$ values of shape-optimized calibration curves (RL and RLBr) as well as for specialized ECCs for mono- and di-brominated reagents $\left(\mathrm{Br} \text { and } \mathrm{Br}_{2}\right)^{[124]}$ for THF- $d_{8}$.

\begin{tabular}{cccccccc}
\hline & $\log (\boldsymbol{K})$ & $\boldsymbol{\Delta} \log (\boldsymbol{K})$ & $-\boldsymbol{\alpha}$ & $\boldsymbol{\Delta} \boldsymbol{\alpha}$ & $\mathbf{a v . d e v .}$ & $\max \mathbf{d e v}$. & cor. $\mathbf{R}^{2}$ \\
\hline Solvent: THF- $\boldsymbol{d}_{\mathbf{8}}$ & & & & & & & \\
\hline $\mathrm{RL}$ & -7.05 & 0.0277 & -0.800 & 0.0122 & $\pm 1 \%$ & $\pm 1 \%$ & 1.00 \\
$\mathrm{RLBr}$ & -7.09 & 0.0625 & -0.745 & 0.0293 & $\pm 1 \%$ & $\pm 1 \%$ & 1.00 \\
$\mathrm{Br}^{[124]}$ & -7.12 & 0.0632 & -0.728 & 0.0284 & $\pm 4 \%$ & $\pm 15 \%$ & 0.96 \\
$\mathrm{Br}_{2}{ }^{[124]}$ & -6.80 & 0.0176 & -0.834 & 0.0740 & $\pm 6 \%$ & $\pm 18 \%$ & 0.93 \\
\hline
\end{tabular}

Table 2-14: Calculated relative theoretical errors $\left(\Delta M W_{\text {det,rel }}\right)$ of used model compounds for RL and RLBr ECCs as well as average values.

\begin{tabular}{lccc}
\hline Compound & RL & RLBr & Merge \\
\hline & $\Delta M W[\%]$ & $\Delta M W[\%]$ & $\Delta M W[\%]$ \\
Batylalcohol & 4 & - & 9 \\
$n$ DecCl & 3 & - & 7 \\
$n$ HexCl & 3 & - & 7 \\
$n$ OctCl & 3 & - & 7 \\
$n$ PrBr & - & 8 & 6 \\
$n$ BuBr & - & 9 & 6 \\
$n$ PenBr & - & 9 & 7 \\
$\varnothing$ & $\mathbf{3}$ & $\mathbf{9}$ & 7 \\
\hline
\end{tabular}

The theoretical errors, at least of the RL ECC, are still questionably low. A deviation of up to $8 \%$ seems to be a realistic margin (same as for the DSE ECC in THF- $d_{8}$ ).

\subsubsection{MW Estimation of Alkyl Grignard Reagents from Diffusion Data}

Because the internal reference TPhN was added beforehand, normalized diffusion coefficients could be calculated for all alkyl Grignard reagents for $M W$ predictions via ECCs.

Additionally, utilizing normalized diffusion coefficients, the concentration dependence could be investigated. A tenfold increase in concentration did not lead to a significant change of the normalized diffusion coefficients $\left(\log \left(D_{x, \text { norm }}\right)(n \mathrm{BuMgCl}, 25 \mathrm{mM})=-8.9750 ; \log \left(D_{\mathrm{x}, \mathrm{norm}}\right)(n \mathrm{BuMgCl}\right.$, $250 \mathrm{mM})=-8.9787)$. Only at a concentration of $1 \mathrm{M}$ of $n \mathrm{BuMgCl}$ did the increase in diffusion coefficient exceed theoretical error limits $\left(\log \left(D_{\mathrm{x}, \mathrm{norm}}\right)(n \mathrm{BuMgCl}, 1 \mathrm{M})=-9.0072\right)$. This could be an indication for the (partial) formation of higher aggregates, however, ECC-DOSY has not been rigorously tested for solutions with concentrations higher than $120 \mathrm{mM} .^{[109 a]}$ Hence, the concentration dependence seems negligible in THF at least for the low concentrations investigated herein.

Utilizing the ECCs for RL compounds, $M W$ s were predicted for all measured alkyl Grignard systems. Furthermore, for comparison, MWs were estimated with merged and DSE ECCs. For $n \mathrm{BuMgBr}$ the RLBr ECC as well as the Br ECC provided by Kreyenschmidt ${ }^{[124]}$ and the correctional factor $\left(X_{\text {cor }}\right)$ introduced in section 2.1.4 were utilized for the estimation of $M W$ s. Results for chlorinated and brominated Grignard systems are listed in Table 2-15 and Table 2-16, respectively 
(the complete set of data is given in the appendix: Table 5-40 (EtMgCl); Table 5-41 (iPrMgCl); Table 5-42 ( $n \mathrm{BuMgCl})$; Table 5-43 ( $n \mathrm{HexMgCl})$; Table 5-44 ( $n$ OctMgCl); Table 5-45 ( $n \mathrm{DecMgCl})$; Table 5-46 ( $n \mathrm{BuMgBr}))$.

Table 2-15: $\mathrm{ECC}^{\mathrm{THF}}$ (RL, DSE and merged) were used to predict $M W_{\mathrm{det}}$ of RMgCl in THF- $d_{8}$. The accuracy of the ECC ${ }^{\mathrm{THF}}$ (RL) is in the range of $\pm 8 \%$ (see section 2.3.1.1), of the $\mathrm{ECC}^{\mathrm{THF}}$ (DSE) in the range of $M W_{\mathrm{dif}}= \pm 8 \%$ and of the $\mathrm{ECC}^{\mathrm{THF}}$ (merged) in the range of $M W_{\mathrm{dif}}= \pm 18 \%$. Theoretical errors, as discussed in section 2.1.3, are given as standard deviations for all estimated $M W$ s. All results in this table are from measurements at $25^{\circ} \mathrm{C} . M W_{\text {dif }}$ is given for monomeric $\left[\mathrm{RMgCl}(\mathrm{THF})_{2}\right]$ and dimeric $[\mathrm{RMgClTHF}]_{2}$ for the RL ECC.

\begin{tabular}{lccccc}
\hline & & & & {$\left[\mathbf{R M g C l}(\mathrm{THF})_{2}\right]$} & {$[\mathbf{R M g C l}(\mathbf{T H F})]_{2} \cdot$} \\
\hline & $M W_{\text {det }}$ & $M W_{\text {det }}$ & $M W_{\text {det }}$ & $M W_{\text {dif }}$ & $M W_{\text {dif }}$ \\
& $(\mathrm{merged})[\mathrm{g} / \mathrm{mol}]$ & $(\mathrm{DSE})[\mathrm{g} / \mathrm{mol}]$ & $(\mathrm{RL})[\mathrm{g} / \mathrm{mol}]$ & $(\mathrm{RL})[\%]$ & $(\mathrm{RL})[\%]$ \\
\hline $\mathbf{E t M g C l}$ & $261 \pm 21$ & $250 \pm 13$ & $228 \pm 8$ & $\mathbf{2}$ & 41 \\
$\boldsymbol{i P r M g C l}$ & $262 \pm 21$ & $251 \pm 13$ & $228 \pm 8$ & $\mathbf{8}$ & 53 \\
$\boldsymbol{n B u M g C l}$ & $307 \pm 25$ & $292 \pm 15$ & $255 \pm 9$ & $\mathbf{2}$ & 48 \\
$\boldsymbol{n} \mathbf{H e x M g C l}$ & $366 \pm 31$ & $345 \pm 19$ & $288 \pm 11$ & $\mathbf{0}$ & 51 \\
$\boldsymbol{n O c t M g C l}$ & $405 \pm 35$ & $381 \pm 21$ & $317 \pm 12$ & $\mathbf{0}$ & 55 \\
$\boldsymbol{n D e c M g C l}$ & $445 \pm 39$ & $417 \pm 24$ & $348 \pm 14$ & $-\mathbf{1}$ & 57 \\
\hline
\end{tabular}

Table 2-16: $\mathrm{ECC}^{\mathrm{THF}}$ (RLBr, Br) were used to predict $M W_{\text {det }}$ of $n \mathrm{BuMgBr}$ in THF- $d_{8}{ }^{[124]}$ The accuracy of the ECC ${ }^{\mathrm{THF}}$ (RLBr) is in the range of $\Delta M W_{\text {det,rel }}= \pm 8 \%$ (theoretical; see section 2.3.1.1) and of the ECC ${ }^{\mathrm{THF}}(\mathrm{Br}$ ) is in the range of $M W_{\text {dif }}= \pm 15 \%{ }^{[124]}$ Theoretical errors, as discussed in section 2.1.3, are given as standard deviations for these estimated $M W s$. All results in this table are from measurements at $25^{\circ} \mathrm{C} . M W_{\text {dif }}$ is given for monomeric $\left[n \operatorname{BuMgBr}(\mathrm{THF})_{2}\right]$ and dimeric $[n \mathrm{BuMgBr}(\mathrm{THF})]_{2}$ for the RLBr ECC. Additionally, the correctional factor $X_{\text {cor }}$ as discussed in section 2.1.4 has been utilized to calculate a $M W\left(M D_{\mathrm{W}}\left(\left[n \mathrm{BuMgBr}(\mathrm{THF})_{2}\right]\right)=6.59 \cdot 10^{29} \mathrm{~g} /\left(\mathrm{mol} \cdot \mathrm{m}^{3}\right)\right.$ was used $)$.

\begin{tabular}{|c|c|c|c|c|c|}
\hline & & & & $\begin{array}{c}{\left[n \mathrm{BuMgBr}(\mathrm{THF})_{2}\right]} \\
305 \mathrm{~g} \mathrm{~mol}^{-1}\end{array}$ & $\begin{array}{c}{[n \mathrm{BuMgBr}(\mathrm{THF})]_{2} .} \\
466 \mathrm{~g} \mathrm{~mol}^{-1}\end{array}$ \\
\hline & $M W_{\text {det }}$ & $M W_{\text {det }}$ & $M W_{\mathrm{det}}$ & $M W_{\text {dif }}$ & $M W_{\mathrm{dif}}$ \\
\hline & $(\mathrm{RLBr})[\mathrm{g} / \mathrm{mol}]$ & $(\mathrm{Br})[\mathrm{g} / \mathrm{mol}]$ & $\left(X_{\text {cor }}\right)[\mathrm{g} / \mathrm{mol}]$ & (RLBr) [\%] & (RLBr) [\%] \\
\hline$n \mathrm{BuMgBr}$ & $330 \pm 33$ & $343 \pm 34$ & 362 (merge) & -8 & 41 \\
\hline
\end{tabular}

The mandatory molar van-der-Waals density calculations have also been done and the complete set of data is given in the appendix (see Table 5-47). Except for $n \mathrm{BuMgBr}$, for all herein proposed aggregates errors due to higher $M D_{\mathrm{W}}$ can be excluded. The estimated $M W_{\text {det }}$ were compared to hypothetical monomeric $\left[\mathrm{RMgX}(\mathrm{THF})_{2}\right]$ and dimeric $[\mathrm{RMgX}(\mathrm{THF})]_{2}$ aggregates that are in accordance with the Schlenk equilibrium.

Estimated $M W$ s show the clear preference for monomeric aggregation as $\left[\mathrm{RMgX}(\mathrm{THF})_{2}\right]$ of alkyl Grignard reagents in THF, which confirms the results and consensus reached in many other studies. ${ }^{[136 b, 140 a-c, 140-\mathrm{h}]}$ Since the diffusion coefficients may be averaged, it is difficult to say, if there are other monomeric species coexisting with proposed $\left[\mathrm{RMgX}(\mathrm{THF})_{2}\right]$ which is a possibility, as shown in the Schlenk equilibrium. However, trinuclear molecules which were observed in the ESI experiments or even dimeric aggregation can be disregarded or are extremely low populated. For $i \mathrm{PrMgCl}$ the ECC for RL molecules should not be utilized, as this molecule cannot be described as rod-like. However, calculations using the DSE-ECC led to similarly good results for monomeric 
aggregation of $\left[i \operatorname{PrMgCl}(\mathrm{THF})_{2}\right]$ as for the other Grignard systems $\left(M W_{\text {dif }}(\mathrm{DSE})=-2 \%\right.$; see Table 5-41 in the appendix).

A special case in this investigation was $n \mathrm{BuMgBr}$. Its proposed aggregates exhibit increased vander-Waals densities (see Table 5-39 in the appendix). Therefore, specialized ECCs for brominated systems ( $\mathrm{RLBr}$ and $\mathrm{Br}$ of Kreyenschmidt ${ }^{[124]}$ ) were utilized to describe this aggregate. These produce almost identical $M W$ s which fit best $\left[n \operatorname{BuMgBr}(\mathrm{THF})_{2}\right]$. Also, the correctional factor $\left(X_{\text {cor }}\right)$ for molecules with increased $M D_{\mathrm{W}}$ was calculated according to the procedure described in section 2.1.4 $\left(X_{\text {cor }}=1.005796\right.$; with $\left.M D_{\mathrm{W}}\left(\left[n \mathrm{BuMgBr}(\mathrm{THF})_{2}\right]\right)=6.59 \cdot 10^{29} \mathrm{~g} /\left(\mathrm{mol} \cdot \mathrm{m}^{3}\right)\right)$ and applied which resulted in a slightly raised but similar $M W$. The increase in $M W$ is reasonable, as no shape-optimization has been done and the corrected diffusion coefficient was applied to the merged ECC. However, if the corrected diffusion coefficient $\left(\log \left(D_{\mathrm{x}, \mathrm{norm}, \mathrm{cor}}\right)(n \mathrm{BuMgBr})=-9.0180\right)$ is applied to the RL ECC, a MW of $288 \mathrm{~g} / \mathrm{mol}$ is obtained, which fits even better the proposed $\left[n \mathrm{BuMgBr}(\mathrm{THF})_{2}\right]^{\mathrm{a}}$

\subsubsection{Solution State Structures of the "Turbo"-Grignard Analogues}

More recently, Grignard reagents were further advanced by Knochel et al. by the investigation of metal halides on their reactivity. ${ }^{[142]}$ The addition of metal halides, e.g. LiCl, led to an immense increase in reactivity, accompanied by an enhanced functional group tolerance. These reagents were accordingly named "turbo"-Grignards and have been largely utilized in different fields across academia and industry ever since. ${ }^{[143]}$ Knochel et al. proposed that this increased reactivity was a result of the formation of ate complexes (see Scheme 2-4). This proposal could be further substantiated by quantum chemical calculations. These also suggested the formation of solvent-separated ion pairs. ${ }^{[144]}$

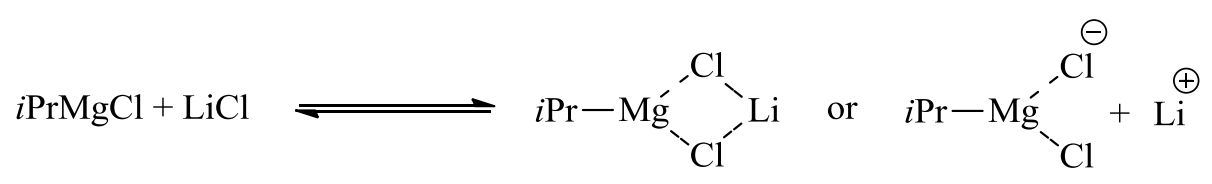

assumed species in solution

Scheme 2-4: $[i \mathrm{PrMgCl} \cdot \mathrm{LiCl}]$ species assumed to be present in solution (ate complexes) ${ }^{\left[{ }^{[142 a}, 144\right]}$ Coordinating solvent molecules are omitted for clarity.

Lerner et al. tried to crystallize "turbo"-Grignard compounds, i.e. [iPrMgCl $\cdot \mathrm{LiCl}]$, but only found the open-cube analogue for $\mathrm{RMgCl}$ (see Scheme 2-3).[141b] This indicates an exchange between conventional RMgX and the lithiated ate species. Since the conventional RMgX were investigated

\footnotetext{
${ }^{a}$ While this approach seems feasible here, note that the correctional factor is based on the merged ECCs and therefore applying the corrected diffusion coefficients to other ECCs has not been proven, yet.

${ }^{b}$ Some results of this section have been published separately: C. Schnegelsberg, S. Bachmann, M. Kolter, T. Auth, M. John, D. Stalke, K. Koszinowski, Chem. Eur. J. 2016, 22, 7752-7762. ${ }^{[3]}$
} 
in the previous section, description of the effect of LiX in solution on these aggregates would be an interesting addition. Hence, "turbo"-analogues were investigated by the same analytical methods (e.g. ESI-MS) as RMgX in cooperation with the Koszinowski group. a As for NMR spectroscopy, the addition of one equivalent of $\mathrm{LiCl}$ to solutions of $n \mathrm{BuMgCl}$ did not change its ${ }^{1} \mathrm{H}$ or ${ }^{13} \mathrm{C}$ spectra in any significant way. ${ }^{7} \mathrm{Li} \mathrm{NMR}$ displayed only a single resonance at $\delta=0.28 \mathrm{ppm}$, which is slightly different to the chemical shift of pure $\mathrm{LiCl}$ in THF $(\delta=0.49 \mathrm{ppm})$. However, ${ }^{1} \mathrm{H}$-DOSY measurements resulted in significantly raised normalized diffusion coefficients for $[n \mathrm{BuMgCl} \cdot \mathrm{LiCl}]\left(\log \left(D_{\mathrm{x}, \mathrm{norm}}\right)([n \mathrm{BuMgCl} \cdot \mathrm{LiCl}])=-9.0293\right)$ compared to the conventional $n \mathrm{BuMgCl}\left(\log \left(D_{\mathrm{x}, \text { norm }}\right)(n \mathrm{BuMgCl})=-8.9750\right)$. This is consistent with findings from Pöppler concerning $i \mathrm{PrMgCl} \cdot \mathrm{LiCl}^{[32 \mathrm{c}]}$ Hence, (partial) association of $\mathrm{LiCl}$ with the Grignard reagents can be concluded which is a confirmation of at least partial formation of the proposed ate complexes of Knochel et al. or neutral complexes shown in Scheme 2-4. ${ }^{[142 a, 144]}$ The estimated MWs for RL, DSE and merge ECCs are shown in Table 2-17 for $[n \mathrm{BuMgCl} \cdot \mathrm{LiCl}]$ (the complete set of data is given in the appendix: Table 5-48).

Table 2-17: ECC ${ }^{\mathrm{THF}}$ (RL, DSE and merged) were used to predict $M W_{\text {det }}$ of $[n \mathrm{BuMgCl} \cdot \mathrm{LiCl}]$ in THF- $d_{8}$. The accuracy of the $\mathrm{ECC}^{\mathrm{THF}}(\mathrm{RL})$ is in the range of $\pm 8 \%$ (see section 2.3.1.1); of the ECC ${ }^{\mathrm{THF}}$ (DSE) is in the range of $M W_{\mathrm{dif}}= \pm 8 \%$ and of the $\mathrm{ECC}^{\mathrm{THF}}$ (merged) in the range of $M W_{\mathrm{dif}}= \pm 18 \%$. Theoretical errors, as discussed in section 2.1.3, are given as standard deviations for all estimated $M W$ s. All results in this table are from measurements at $25^{\circ} \mathrm{C} . M W_{\text {dif }}$ is given for monomeric $\left[n \mathrm{BuMgCl}(\mathrm{THF})_{3} \cdot \mathrm{LiCl}\right]$ for the DSE ECC.

\begin{tabular}{ccccc}
\hline & & & $\begin{array}{c}{\left[\mathbf{n B u g C l}(\mathrm{THF})_{3} \cdot \mathbf{L i C l}\right]} \\
\mathbf{3 7 6} \mathbf{~ g ~ m o l}^{-1}\end{array}$ \\
\hline & $\begin{array}{c}M W_{\text {det }} \\
(\mathrm{RL})[\mathrm{g} / \mathrm{mol}]\end{array}$ & $\begin{array}{c}M W_{\text {det }} \\
(\mathrm{DSE})[\mathrm{g} / \mathrm{mol}]\end{array}$ & $\begin{array}{c}M W_{\text {det }} \\
(\mathrm{Merge})[\mathrm{g} / \mathrm{mol}]\end{array}$ & $\begin{array}{c}M W_{\text {dif }} \\
(\mathrm{DSE})[\%]\end{array}$ \\
\hline$[\mathbf{n B u M g C l} \cdot \mathbf{L i C l}]$ & $330 \pm 11$ & $362 \pm 20$ & $384 \pm 33$ & 4 \\
\hline
\end{tabular}

Results from the RL ECC must be interpreted with caution, as the addition of $\mathrm{LiCl}$ to the structure would certainly result in a smaller influence of the alkyl chain to the overall shape of the solute compared to conventional RMgX. While the RL ECC results in an estimated $M W$ that corresponds to $\left[n \mathrm{BuMgCl}(\mathrm{THF})_{2} \cdot \mathrm{LiCl}\right]$ which has a slightly increased $M D_{\mathrm{W}}$ and is not in agreement with the coordination number four for either magnesium or lithium, the results of the DSE ECCs are more feasible. Hence, the solution state structure $\left[n \mathrm{BuMgCl}(\mathrm{THF})_{3} \cdot \mathrm{LiCl}\right]$ is proposed, which fits the expectations. $^{\mathrm{b}}$

The brominated $n \mathrm{BuMgBr}$ also exhibited elevated diffusion coefficients after addition of $\mathrm{LiBr}$ $\left(\log \left(D_{\mathrm{x}, \mathrm{norm}}\right)([n \mathrm{BuMgBr} \cdot \mathrm{LiBr}])=-8.9816 ; \log \left(D_{\mathrm{x}, \mathrm{norm}}\right)(n \mathrm{BuMgBr})=-8.9660\right)$ which indicates a (partial) association of the solutes. MWs were estimated with the specialized $\mathrm{Br}_{2}$ ECC of Kreyenschmidt. ${ }^{[124]}$ Moreover, calculations with the correctional factor $X_{\text {cor }}$ were made $\left(X_{\text {cor }}=\right.$

a For a detailed description of results from ESI-MS, electrical conductivity measurements and quantum chemical calculations consult reference [3].

b ${ }^{7}$ Li-DOSY was not measured. 
1.007461 ; presuming the formation of $\left[n \mathrm{BuMgBr}(\mathrm{THF})_{3} \cdot \mathrm{LiBr}\right] \quad$ with $M D_{\mathrm{W}}\left(\left[n \mathrm{BuMgBr}(\mathrm{THF})_{3} \cdot \mathrm{LiBr}\right]=7.07 \cdot 10^{29} \mathrm{~g} /\left(\mathrm{mol} \cdot \mathrm{m}^{3}\right)\right)$. Results are shown in Table 2-18 (the complete set of data is given in the appendix: Table 5-49).

Table 2-18: $\mathrm{ECC}{ }^{\mathrm{THF}}\left(\mathrm{Br}_{2}\right)$ was used to predict $M W_{\text {det }}$ of $[n \mathrm{BuMgBr} \cdot \mathrm{LiBr}]$ in THF- $d_{8}{ }^{[124]}$ The accuracy of the $\mathrm{ECC}^{\mathrm{THF}}\left(\mathrm{Br}_{2}\right)$ is in the range of $M W_{\mathrm{dif}}= \pm 18 \%$. ${ }^{[124]}$ Theoretical errors, as discussed in section 2.1.3, are given as standard deviations for these estimated $M W$. All results in this table are from measurements at $25^{\circ} \mathrm{C} . M W_{\text {dif }}$ is given for monomeric $\left[n \mathrm{BuMgBr}(\mathrm{THF})_{3} \cdot \mathrm{LiBr}\right]$ and $\left[n \mathrm{BuMgBr}(\mathrm{THF})_{2} \cdot \mathrm{LiBr}\right]$ for the $\mathrm{Br}_{2}$ ECC. Additionally, the correctional factor $X_{\text {cor }}$ as discussed in section 2.1.4 has been utilized to calculate a $M W\left(M D_{\mathrm{W}}\right.$ of $\left[n \mathrm{BuMgBr}(\mathrm{THF})_{3} \cdot \mathrm{LiBr}\right]$ was used).

\begin{tabular}{|c|c|c|c|c|}
\hline & & & $\begin{array}{c}{\left[n \mathrm{BuMgBr}(\mathrm{THF})_{3} \cdot \mathrm{LiBr}\right]} \\
465 \mathrm{~g} \mathrm{~mol}^{-1}\end{array}$ & $\begin{array}{c}{\left[n \mathrm{BuMgBr}(\mathrm{THF})_{2} \cdot \mathrm{LiBr}\right]} \\
392 \mathrm{~g} \mathrm{~mol}^{-1}\end{array}$ \\
\hline & $\begin{array}{c}M W_{\text {det }} \\
\left(\mathrm{Br}_{2}\right)[\mathrm{g} / \mathrm{mol}]\end{array}$ & $\begin{array}{c}M W_{\text {det }} \\
\left(X_{\text {cor }}\right)[\mathrm{g} / \mathrm{mol}]\end{array}$ & $\begin{array}{c}M W_{\mathrm{dif}} \\
\left(\mathrm{Br}_{2}\right)[\%]\end{array}$ & $\begin{array}{c}M W_{\mathrm{dif}} \\
\left(\mathrm{Br}_{2}\right)[\%]\end{array}$ \\
\hline$[n \mathrm{BuMgBr} \cdot \mathrm{LiBr}]$ & $413 \pm 96$ & 412 (merge) & 12 & -5 \\
\hline
\end{tabular}

These results point towards the formation of $\left[n \mathrm{BuMgBr}(\mathrm{THF})_{3} \cdot \mathrm{LiBr}\right]$ or $\left.\left[n \mathrm{BuMgBr}(\mathrm{THF})_{2} \cdot \mathrm{LiBr}\right]\right]^{\mathrm{a}}$ However, for the $\mathrm{Br}_{2}$ calibration curve as well as for the correctional factor, the assumption of the coordination of $\mathrm{LiBr}$ was already made a priori. Hence, these results should be weighted even more carefully than in the case of the chlorinated reagents. Furthermore, the exact solvation state is difficult to assign, as the errors of the ECCs and correctional factor exceed the deviations of the different species. It is always possible that the diffusion coefficients are averaged as some part of an equilibrium, however, as $\left[n \mathrm{BuMgBr}(\mathrm{THF})_{3} \cdot \mathrm{LiBr}\right]$ would invoke lithium's and magnesium's preferred coordination number four, it is more likely from a chemical point of view.

In conclusion, while the aggregation of these "turbo"-Grignard reagents cannot be described without uncertainty, the increase in diffusion coefficient upon addition of LiX salts corroborates the formation of ate complexes proposed by Knochel et al. in the past. ${ }^{[142 a, 144]}$

\subsubsection{Anion and Solvent Coordination Elucidated via DOSY}

The ECC technique is mostly restricted to hydrocarbons and less sensitive when considering transition metal complexes due to problems related to elevated $M D_{\mathrm{W}}$ or shape irregularities. However, DOSY and diffusion coefficient normalization in general are useful tools to investigate even such molecules. This will be demonstrated and discussed in this section while investigating the coordination of different anions to an $\mathrm{N}$-heterocyclic carbene precursor. Additionally, the solvation of an acridine-based fluorophore and a heterocyclic substituted methanide and their metal complexes are elucidated by diffusion NMR techniques.

${ }^{\text {a }}$ Note that no shape dependence can be discussed for the utilized ECC and correctional factor. 


\subsubsection{Coordination of Different Anions to an N-Heterocyclic Carbene in $\mathrm{CD}_{2} \mathrm{Cl}_{2}{ }^{\mathrm{a}}$}

The synthesis of stable $\mathrm{N}$-heterocyclic carbenes (NHCs), ${ }^{[146]}$ which are widely used as ligands in transition metal chemistry and can even be used as powerful organocatalysts, has been an important addition to modern organometallic chemistry. Their metal complexes frequently display enhanced catalytic activity, e.g. Grubbs' second generation catalyst for olefin metathesis. ${ }^{[147]}$ Recently, the synthesis of unconventional carbenes, i.e. mesoionic carbenes, has come to the focus of researchers, as such compounds have an even higher $\sigma$-donor strength. ${ }^{[148]}$ Hence, new synthetic approaches were made by Reichmann to widen the scope of such unconventional carbenes. He

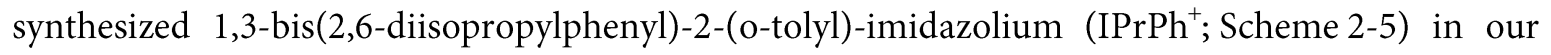
group as precursor in this pursuit. To enhance its solubility and this way optimize follow-up reactions the corresponding anions were varied, i.e. strongly coordinating halides $\left(\mathrm{Br}^{-}, \mathrm{I}^{-}\right)$and less coordinating fluorinated anions $\left(\mathrm{BF}_{4}^{-}, \mathrm{PF}_{6}^{-}, \mathrm{OTf}^{-}\right)$were used.

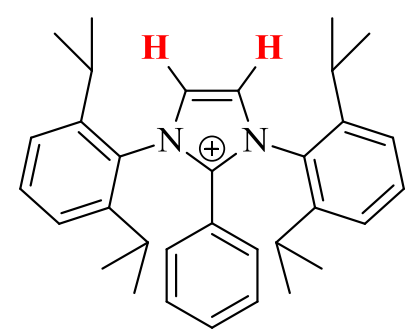

Scheme 2-5: Structure of 1,3-bis(2,6-diisopropylphenyl)-2-(o-tolyl)-imidazolium ( $\left.\mathrm{PPrPh}^{+}\right)$.

The variation of anions had a visible effect on the proton spectra of these compounds in $\mathrm{CD}_{2} \mathrm{Cl}_{2}$, as the signal of the back-bone protons (marked red in Scheme 2-5) of $\mathrm{IPrPh}^{+}$changed significantly $(\delta$ $\left.=8.29 \mathrm{ppm}\left(\mathrm{Br}^{-}\right) ; 8.07 \mathrm{ppm}\left(\mathrm{I}^{-}\right) ; 7.97 \mathrm{ppm}\left(\mathrm{OTf}^{-}\right) ; 7.88 \mathrm{ppm}\left(\mathrm{BF}_{4}^{-}\right) ; 7.76 \mathrm{ppm}\left(\mathrm{PF}_{6}^{-}\right)\right)$, while all other signals remained identical. ${ }^{[145]}$ Reichmann correlated this chemical shift difference to the acidity of the protons and proposed that the brominated species is most acidic, whereas the compound with $\mathrm{PF}_{6}{ }^{-}$is the least acidic. ${ }^{[145]}$ The assumption that the fluorinated anions would be less likely coordinated to the $\mathrm{IPrPh}^{+}$could be dismissed by ${ }^{19} \mathrm{~F},{ }^{1} \mathrm{H}$-HOESY experiments which showed in all three cases couplings between the anions and the backbone protons of the precursor. $^{[145]}$

ECC-DOSY has then been utilized to further elucidate structural motifs: Adamantane (Adam) was used as internal reference and samples were prepared of each derivative in $\mathrm{CD}_{2} \mathrm{Cl}_{2}$. MWs were estimated utilizing the DSE and merged ECCs, as the molecules can be described neither as flat nor purely spherical. Results are shown in Table 2-19 (the complete set of data is given in the appendix:

\footnotetext{
a Some of the results have been published separately: S. O. Reichmann, Unconventional Carbene-Donor Ligands for the Development of New Catalysts, Ph. D. thesis, Göttingen, 2016. ${ }^{[145]}$ Furthermore, syntheses, NMR assignments and crystal structures of the individual reagents can be found there.
} 
Table 5-51 ((IPrPh)Br); Table 5-52 ((IPrPh)I); Table 5-53 ((IPrPh)OTf); Table 5-54 ((IPrPh)BF $)$; Table 5-55((IPrPh) $\left.\left.\mathrm{PF}_{6}\right)\right)$.

The predicted $M W$ s were compared to $M W_{\text {calc }}$ of species with and without coordinated anions and in all cases this comparison concludes the anion's coordination. Theoretical error ranges further disprove the possibility that some anions might not coordinate at all. Hence, these observations are in accordance with the ${ }^{19} \mathrm{~F},{ }^{1} \mathrm{H}-\mathrm{HOESY}$ experiments.

Table 2-19: ECC $\mathrm{CD}^{\mathrm{CC} 12}$ (DSE and merged) were used to predict $M W_{\text {det }}$ of (IPrPh)Br, (IPrPh)I, (IPrPh)OTf, (IPrPh)BF $\mathrm{BF}_{4}$ and (IPrPh) $\mathrm{PF}_{6}$ in $\mathrm{CD}_{2} \mathrm{Cl}_{2}$. The accuracy of the $\mathrm{ECC}^{\mathrm{CD} 2 \mathrm{Cl} 2}$ (DSE) is in the range of $M W_{\mathrm{dif}}= \pm 14 \%$ and of the $\mathrm{ECC}^{\mathrm{CD} 2 \mathrm{Cl} 2}$ (merged) in the range of $M W_{\text {dif }}= \pm 23 \%$. Theoretical errors, as discussed in section 2.1.3, are given as standard deviations for all estimated $M W$ s. All results in this table are from measurements at $25^{\circ} \mathrm{C} . M W_{\text {dif }}$ is always given for (IPrPh) ${ }^{+}$and the corresponding aggregate with the respective coordinated anion for the DSE ECC.

\begin{tabular}{lcccc}
\hline & & & $\begin{array}{c}\mathbf{I P r P h}^{+} \\
\mathbf{4 6 6} \mathbf{g} / \mathbf{m o l}\end{array}$ & with anion \\
\hline & $\begin{array}{c}M W_{\text {det }} \\
(\mathrm{merged})[\mathrm{g} / \mathrm{mol}]\end{array}$ & $\begin{array}{c}M W_{\text {det }} \\
(\mathrm{DSE})[\mathrm{g} / \mathrm{mol}]\end{array}$ & $\begin{array}{c}M W_{\text {dif }} \\
(\mathrm{DSE})[\%]\end{array}$ & $\begin{array}{c}M W_{\text {dif }} \\
(\mathrm{DSE})[\%]\end{array}$ \\
\hline (IPrPh)Br & $661 \pm 75$ & $590 \pm 60$ & -21 & $-\mathbf{8}$ \\
$(\mathbf{I P r P h}) \mathbf{I}$ & $614 \pm 69$ & $551 \pm 55$ & -15 & $\mathbf{8}$ \\
$(\mathbf{I P r P h}) \mathbf{O T f}$ & $629 \pm 71$ & $564 \pm 57$ & -17 & $\mathbf{9}$ \\
$(\mathbf{I P r P h}) \mathbf{B F}_{4}$ & $641 \pm 73$ & $573 \pm 58$ & -19 & $-\mathbf{4}$ \\
$(\mathbf{I P r P h}) \mathbf{P F}_{6}$ & $631 \pm 71$ & $565 \pm 57$ & -18 & $\mathbf{8}$ \\
\hline
\end{tabular}

Still, taking a closer look at the predicted $M W$ s, there are some questions remaining that should be discussed: These aggregates display almost identical normalized diffusion coefficients (e.g. $\left.\log \left(D_{\mathrm{x}, \mathrm{norm}}\right)((\mathrm{IPrPh}) \mathrm{I})=-9.0416 ; \log \left(D_{\mathrm{x}, \mathrm{norm}}\right)\left((\mathrm{IPrPh}) \mathrm{PF}_{6}\right)=-9.0480\right)$. Hence, it is difficult to compare absolute values, as their differences mostly do not transcend error ranges. However, it should be possible to rationalize tendencies, if the anions had equal influence on the overall structure, i.e. coordinate equally strong. The decrease in diffusion coefficient should then be proportional to the increase of the van-der-Waals volumes of the anions. This does not seem to be the case, as the van-der-Waals volumes of the anions are related like $\operatorname{Br}^{-}\left(2.65 \cdot 10^{-29} \mathrm{~m}^{3}\right)>\mathrm{I}^{-}$ $\left(3.25 \cdot 10^{-29} \mathrm{~m}^{3}\right)>\mathrm{BF}_{4}^{-}\left(8.29 \cdot 10^{-29} \mathrm{~m}^{3}\right)>\mathrm{PF}_{6}^{-}\left(1.04 \cdot 10^{-28} \mathrm{~m}^{3}\right)>\mathrm{OTf}^{-}\left(1.29 \cdot 10^{-28} \mathrm{~m}^{3}\right)$, whereas the diffusion coefficients and the resulting $M W$ s are not (see Table 2-19). This could have several reasons: Either the anions do not have enough impact on the overall structure, and their differences are simply compensated by the error of the experiment, or the degree of coordination varies for each anion which therefore results in diffusion values that do not correlate directly with the anion's volumes. Whichever it is, cannot be unequivocally determined. Furthermore, elevated molecular densities $\left(M D_{\mathrm{W}}\right)$ can lead to the underestimation of some $M W \mathrm{~s}$. However, here this is only the case for the iodated reagent (the complete set of data is given in the appendix: Table 5-56).

Additionally, it must be noted that $\mathrm{IPrPh}^{+}$has sterically demanding groups, i.e. $i \mathrm{Pr}$ and $\mathrm{Ph}$, which can rotate and therefore exhibit increased hydrodynamic radii which is not covered by the existing power law variations. Hence, diffusion coefficients for all these molecules might be overestimated. 
What degree of overestimation this would produce is difficult to predict. However, this should be comparable for all aggregates. In the future, possibly theoretical calculations may enlighten these observations.

\subsubsection{Solvent-Anion Exchange of an Acridine-Based System in DMF- $d_{7}{ }^{a}$}

The detection and/or quantification of specific ions in solution, e.g. $\mathrm{Zn}^{2+}$ and $\mathrm{Cd}^{2+}$ in the human body, is highly relevant, as their concentration can be an important diagnostic tool or in other cases even their occurrence can have harmful effects. ${ }^{[149]}$ Hence, the synthesis of sensor molecules designed to fulfil that need is a rewarding goal. In our group Visscher recently synthesized an acridine-based fluorophore (4,5-bis-( $N, N$-dimethylaminemethylene)-acridine; see Scheme 2-6) which shows selective $\mathrm{Zn}^{2+}$ and $\mathrm{Cd}^{2+}$ ion binding together with a detectable fluorescence response. ${ }^{[1,}$ 150]

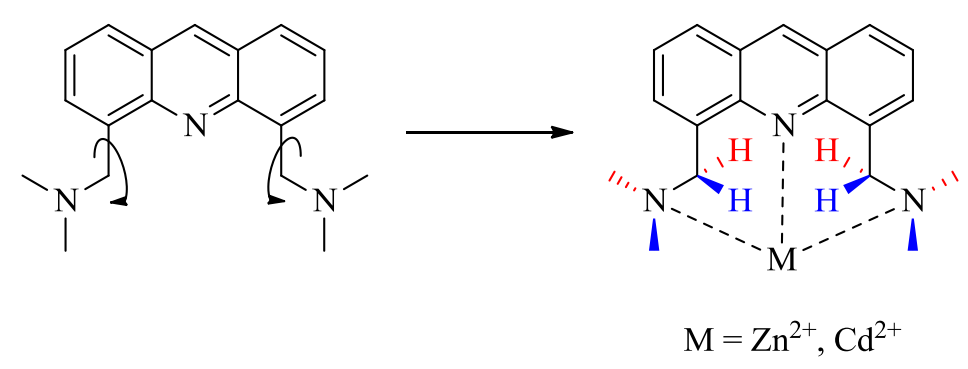

Scheme 2-6: Structure of 4,5-bis-( $N, N$-dimethylaminemethylene)-acridine) and its metal complexes. The possibility of rotation of the amines is hindered after metal coordination. Highlighted groups (red and blue) then lose their chemical equivalency.

In his work, Visscher was able to show that after metal binding the molecule fluoresces. He further characterized the ligand itself as well as the resulting $\mathrm{Zn}^{2+}$ and $\mathrm{Cd}^{2+}$ metal complexes with different analytical techniques (X-ray diffraction, ESI-TOF mass spectrometry, fluorescence spectroscopy). For a more detailed look at these results as well as a description of the synthesis of bis $(\mathrm{N}, \mathrm{N}-$ dimethylaminemethylene)acridine see the corresponding publication. ${ }^{[1,150]}$ Since it is important for a solution-based sensor to understand its solution state structure, the ligand system and the metal complexes were also investigated by NMR and the corresponding findings will be highlighted in this section. Visscher recorded proton spectra of the three compounds $\left(\mathrm{Me}_{2} \mathrm{NCH}_{2}\right)_{2} \mathrm{Acr}$, $\left.\left[\mathrm{ZnBr}(\mathrm{DMF})\left(\left(\mathrm{Me}_{2} \mathrm{NCH}_{2}\right)_{2} \mathrm{Acr}\right)\right)\right]^{+}$and $\left.\left[\mathrm{CdBr}_{2}\left(\left(\mathrm{Me}_{2} \mathrm{NCH}_{2}\right)_{2} \mathrm{Acr}\right)\right)\right]^{\mathrm{b}}$ by dissolving their crystals in DMSO- $d_{6}$. While the spectrum of the ligand showed the expected signal set, the resonances of the metal complexes were highly broadened at $25^{\circ} \mathrm{C}$ (for $\left.\left[\mathrm{CdBr}_{2}\left(\left(\mathrm{Me}_{2} \mathrm{NCH}_{2}\right)_{2} \mathrm{Acr}\right)\right)\right]$ only the non-aromatic signals were broadened). Due to this broadening a sensible DOSY analysis was impossible at this temperature. Therefore, in cooperation with Visscher, the solvent was changed to

\footnotetext{
a Some results of this section have been published separately: A. Visscher, S. Bachmann, C. Schnegelsberg, T. Teuteberg, R. A. Mata, D. Stalke, Dalton Trans. 2016, 45, 5689-5699. [1]

b Sum formulae from crystal structures, for further information see reference [1].
} 
DMF- $d_{7}$, which made a wider temperature range accessible $\left(-61\right.$ to $\left.151^{\circ} \mathrm{C}\right)$. Even though signals were still broadened at $25^{\circ} \mathrm{C}$ in this solvent, temperature-dependent NMR revealed at elevated temperatures $\left(80^{\circ} \mathrm{C}\right)$ one definite signal set for both metal complexes, which was similar, but shifted compared to the ligand (for NMR assignments see section 4.2.7). Cooling the samples led to a splitting of the signals of the diastereotopic methyl groups and methylene protons (marked red and blue in Scheme 2-6) below the coalescence temperature (about $30^{\circ} \mathrm{C}$ for the $\mathrm{Zn}^{2+}$ complex and $40{ }^{\circ} \mathrm{C}$ for the $\mathrm{Cd}^{2+}$ complex; could be slightly different for methyl and methylene groups). This dynamic behavior explains their broadened resonances at ambient temperature. The temperature-dependent measurements (from $80^{\circ} \mathrm{C}$ to $-40{ }^{\circ} \mathrm{C}$ ) are shown for the $\mathrm{Cd}^{2+}$ complex in Figure 2-27 and for the $\mathrm{Zn}^{2+}$ complex in Figure 2-29.

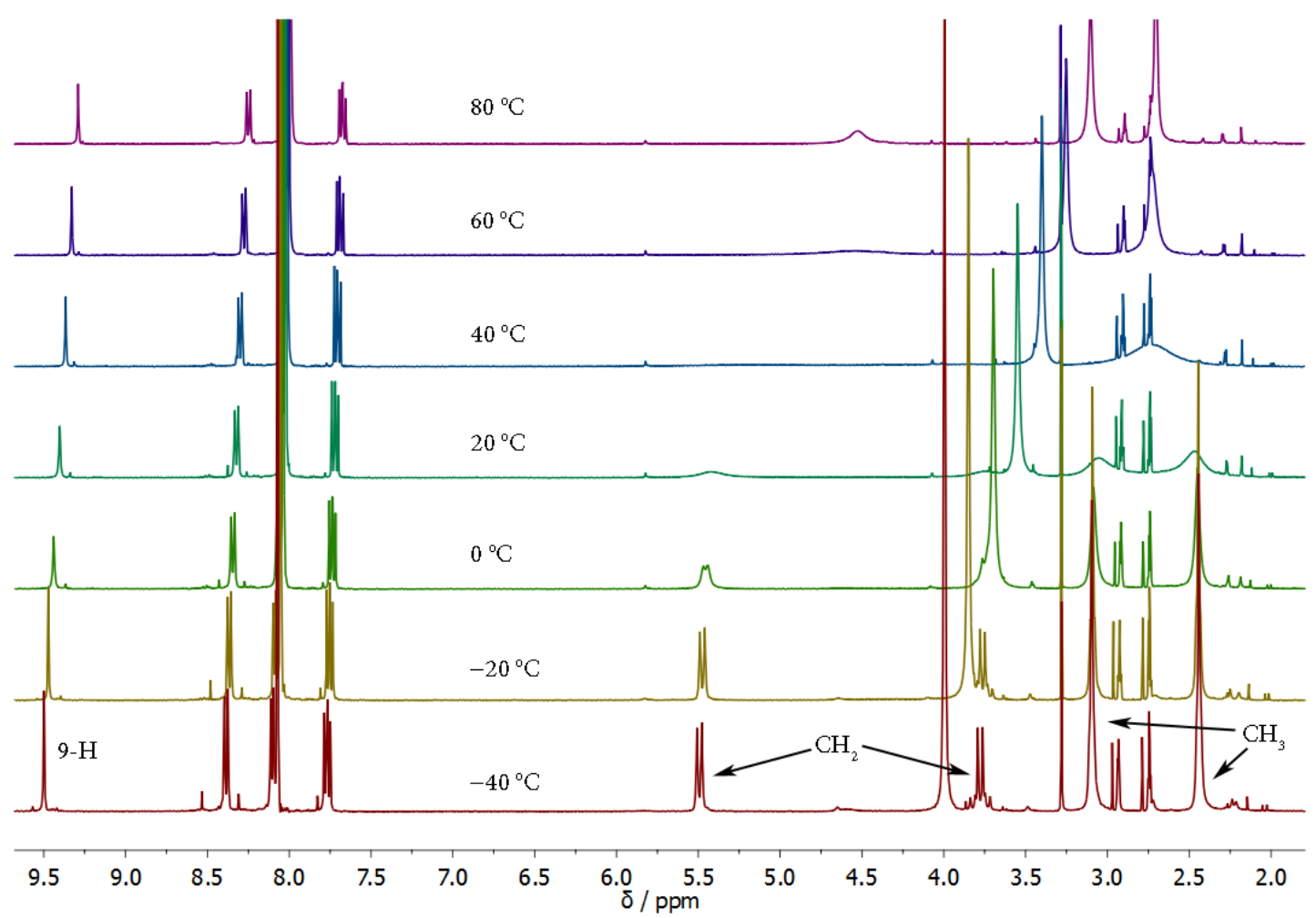

Figure 2-27: Temperature-dependent ${ }^{1} \mathrm{H}$ NMR spectra of $\left.\left[\mathrm{CdBr}_{2}\left(\left(\mathrm{Me}_{2} \mathrm{NCH}_{2}\right)_{2} \mathrm{Acr}\right)\right)\right]$ in DMF- $d_{7}$ from $80{ }^{\circ} \mathrm{C}$ to $-40^{\circ} \mathrm{C}$. Coalescence temperature is at about $40{ }^{\circ} \mathrm{C}$.

To fully ascertain the ligand binding, further experiments were conducted: For $\left.\left[\mathrm{CdBr}_{2}\left(\left(\mathrm{Me}_{2} \mathrm{NCH}_{2}\right)_{2} \mathrm{Acr}\right)\right)\right]$ couplings of the NMR-active ${ }^{113} \mathrm{Cd}$ nuclei to the methyl- and methylene-protons could be observed in a ${ }^{113} \mathrm{Cd},{ }^{1} \mathrm{H}-\mathrm{HMBC}$ experiment at $-30{ }^{\circ} \mathrm{C}$ (see Figure 2-28).

The chemical shift of ${ }^{113} \mathrm{Cd}$ was observed at $\delta=-345 \mathrm{ppm}$ (referenced to $\mathrm{Me}_{2} \mathrm{Cd}$ ). Only the coupling of one dihedral proton of each methylene group towards cadmium was visible, possibly due to an unfavourable angle of the related atoms which is important for the observation of vicinal couplings. ${ }^{[151]}$ 


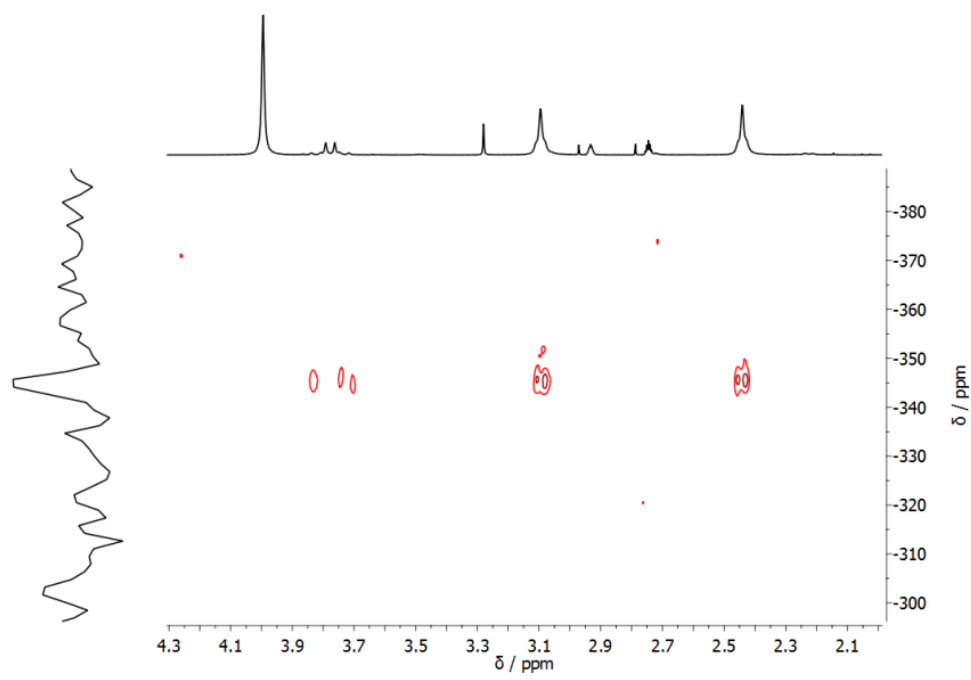

Figure 2-28: ${ }^{113} \mathrm{Cd},{ }^{1} \mathrm{H}-\mathrm{HMBC}$ spectrum of $\left.\left[\mathrm{CdBr}_{2}\left(\left(\mathrm{Me}_{2} \mathrm{NCH}_{2}\right)_{2} \mathrm{Acr}\right)\right)\right]$ in DMF- $d_{7}$ at $-30{ }^{\circ} \mathrm{C}$. Couplings of the ${ }^{113} \mathrm{Cd}$ nuclei to the methyl-groups and one methylene-group are visible.

Since the only NMR active nucleus for zinc $\left({ }^{67} \mathrm{Zn}\right)$ has low natural abundance $(4.1 \%)$ and a large quadrupole moment, since it is a spin $5 / 2$ nucleus, the observation of ${ }^{15} \mathrm{~N}$ chemical shifts and couplings via ${ }^{15} \mathrm{~N},{ }^{1} \mathrm{H}-\mathrm{HMBC}$ experiments was the only feasible way to further attest its binding. A small but significant deshielding of $\delta=8.6 \mathrm{ppm}$ could be observed by comparison of the ${ }^{15} \mathrm{~N}$ chemical shifts of the side-arm nitrogen of the free ligand and the metal complex. ${ }^{[152]}$ Also, for the $\mathrm{Zn}^{2+}$ complex the formation of additional species was observed at lower temperatures which explains why its aromatic signals are also broadened at ambient temperatures in contrast to the $\mathrm{Cd}^{2+}$ complex (see Figure 2-29).

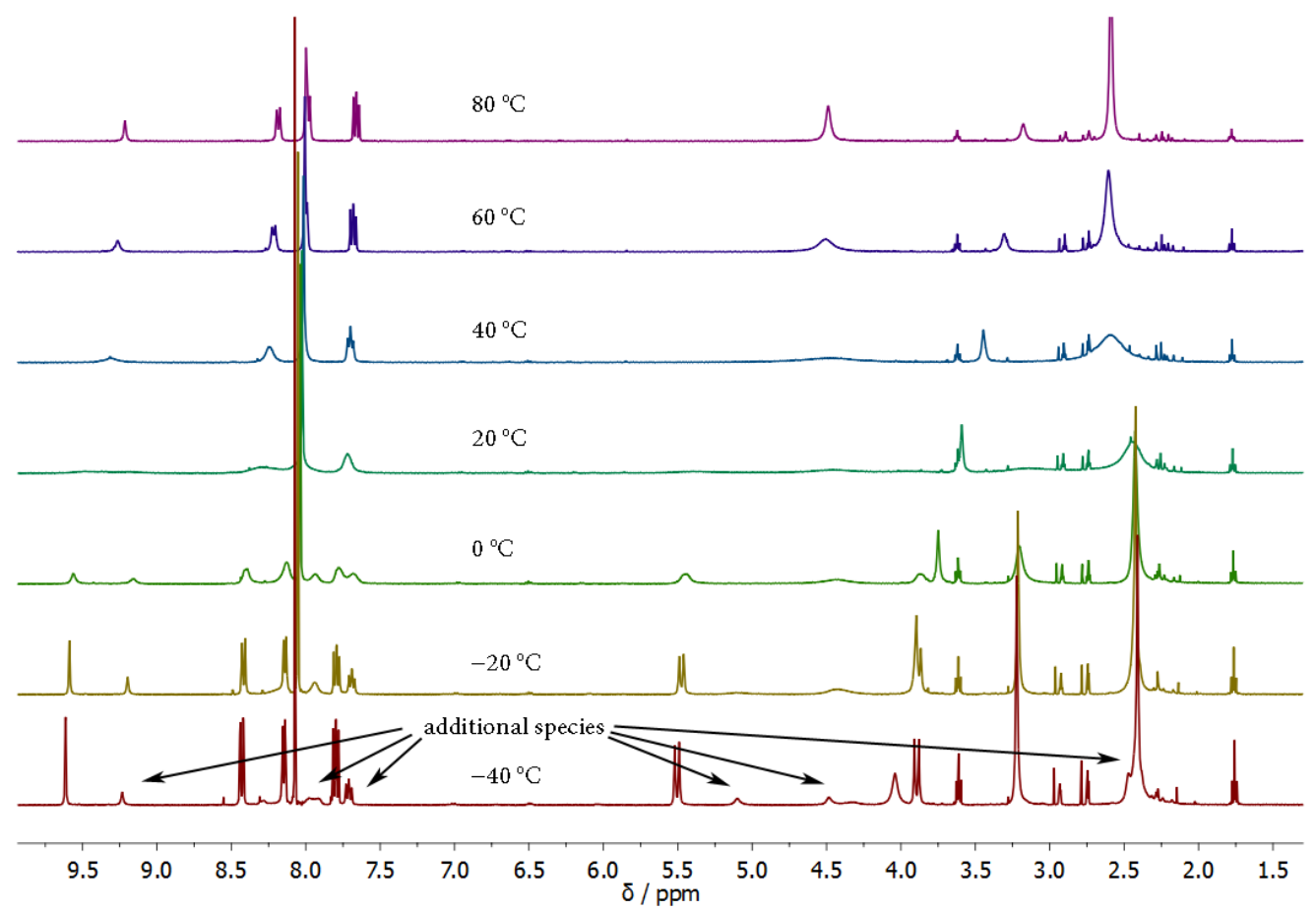

Figure 2-29: Temperature-dependent ${ }^{1} \mathrm{H}$ NMR spectra of $\left.\left[\mathrm{ZnBr}(\mathrm{DMF})\left(\left(\mathrm{Me}_{2} \mathrm{NCH}_{2}\right)_{2} \mathrm{Acr}\right)\right)\right]^{+}$in $\mathrm{DMF}-d_{7}$ from $80^{\circ} \mathrm{C}$ to $-40{ }^{\circ} \mathrm{C}$. Coalescence temperature is at about $30^{\circ} \mathrm{C}$. Additional species can be identified at lower temperatures (with an intensity maximum at about $-20^{\circ} \mathrm{C}$ ). 
Since such species could not be observed at higher temperatures, they seem to originate from dynamical exchange processes that are slowed below the NMR timescale by cooling. A hint towards the origin of this exchange is given by the crystal structures of the metal complexes: While for $\left.\left[\mathrm{CdBr}_{2}\left(\left(\mathrm{Me}_{2} \mathrm{NCH}_{2}\right)_{2} \mathrm{Acr}\right)\right)\right]$ the $\mathrm{Cd}^{2+}$ is coordinated by two bromine atoms, in case of $\left.\left[\mathrm{ZnBr}(\mathrm{DMF})\left(\left(\mathrm{Me}_{2} \mathrm{NCH}_{2}\right)_{2} \mathrm{Acr}\right)\right)\right]^{+}$one of these bromines has been exchanged for a DMF molecule. The stronger binding of the $\mathrm{Br}^{-}$anions towards $\mathrm{Cd}^{2+}$ can be explained by the HSAB theory, as $\mathrm{Cd}^{2+}$ is a softer metal than $\mathrm{Zn}^{2+}$ and hence more prone to bind the softer $\mathrm{Br}^{-}$. Due to this observation from the solid state, it is reasonable to assume that such an exchange of bromine and solvent might also be the reason for the additional species observed at lower temperatures in the NMR spectra of the $\mathrm{Zn}^{2+}$ complex. Hence, to shift the corresponding equilibrium towards the brominated species, a solution of $\mathrm{NaBr}$ was added in excess (10 eq.) to the sample. This addition reduced the broadening of the aromatic signals at $25^{\circ} \mathrm{C}$. Furthermore, the ratio of signal intensities observed at lower temperatures for the different aggregates was reversed after the addition of $\mathrm{NaBr}$ (see Figure 2-30), indicating that the dominant species before has been $\left.\left[\mathrm{Zn}(\mathrm{DMF})_{2}\left(\left(\mathrm{Me}_{2} \mathrm{NCH}_{2}\right)_{2} \mathrm{Acr}\right)\right)\right]^{2+}$.

A closer look at the methylene region of the spectrum reveals the possibility of altogether three distinct, coexisting species (A, B, C; see Figure 2-30), whereas for two of these species most signals seem to overlap. Since the signal intensity of $\mathbf{B}$ and $\mathbf{C}$ rose after the addition of the $\mathrm{NaBr}$ solution, it can be assumed that these signals belong to the brominated derivatives, probably $\left.\left[\mathrm{ZnBr}(\mathrm{DMF})\left(\left(\mathrm{Me}_{2} \mathrm{NCH}_{2}\right)_{2} \mathrm{Acr}\right)\right)\right]^{+}$and $\left.\left[\mathrm{ZnBr}_{2}\left(\left(\mathrm{Me}_{2} \mathrm{NCH}_{2}\right)_{2} \mathrm{Acr}\right)\right)\right]$.

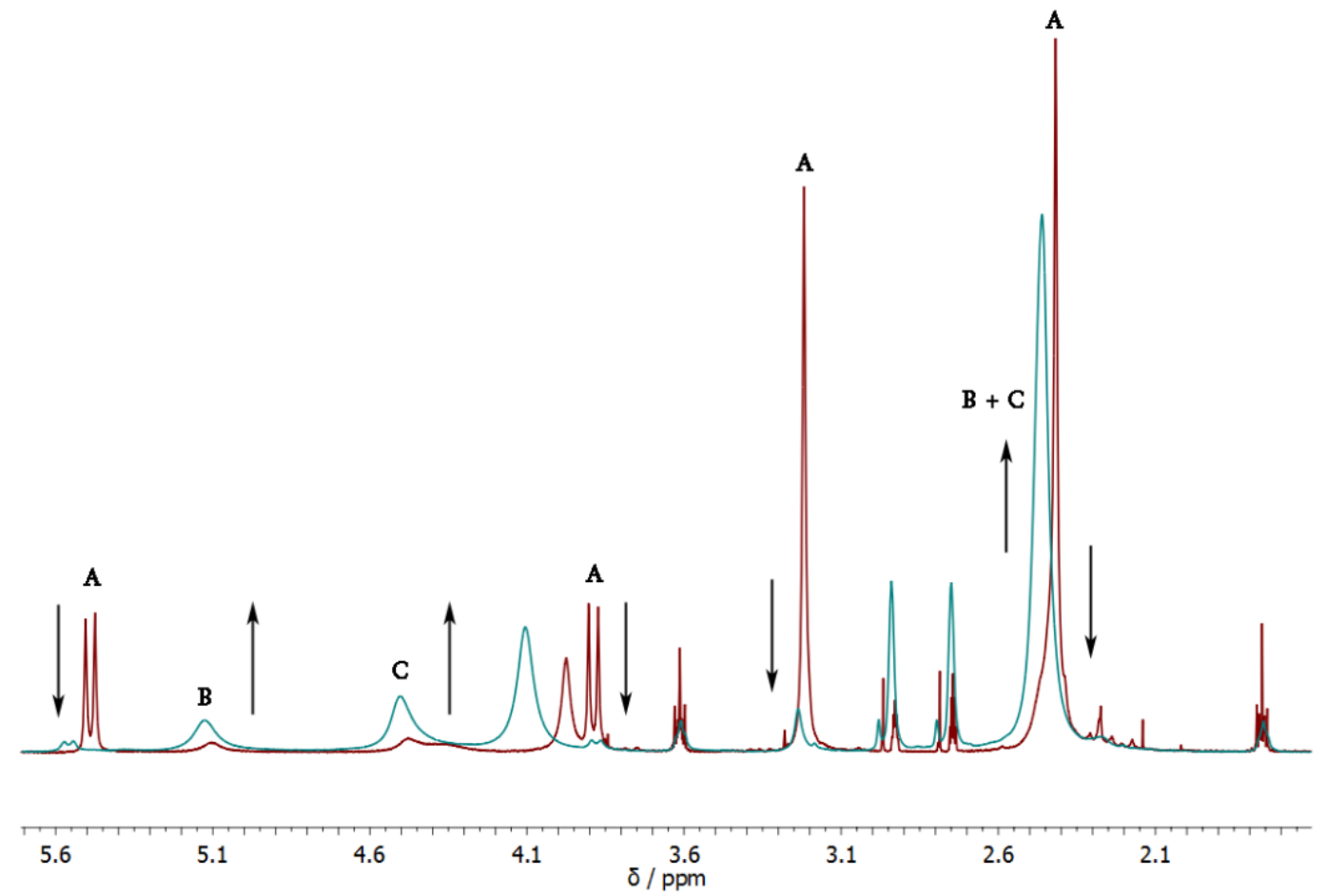

Figure 2-30: Excerpt from the superimposed ${ }^{1} \mathrm{H}$ NMR spectra of $\left.\left[\mathrm{ZnBr}(\mathrm{DMF})\left(\left(\mathrm{Me}_{2} \mathrm{NCH}_{2}\right)_{2} \mathrm{Acr}\right)\right)\right]^{+}$in $\mathrm{DMF}-d_{7}$ at $-30^{\circ} \mathrm{C}$ before (red) and after the addition of $10 \mathrm{eq}$. $\mathrm{NaBr}$ (turquois). The methylene region displays three sets of signals indicating three species (A, B and C). While the signal intensity of $\mathbf{A}$ decreases after the $\mathrm{NaBr}$ addition, it rises for $\mathbf{B} / \mathbf{C}$. 
To confirm this suspicion ${ }^{1} \mathrm{H}$-DOSY NMR was employed (see Figure 2-31).

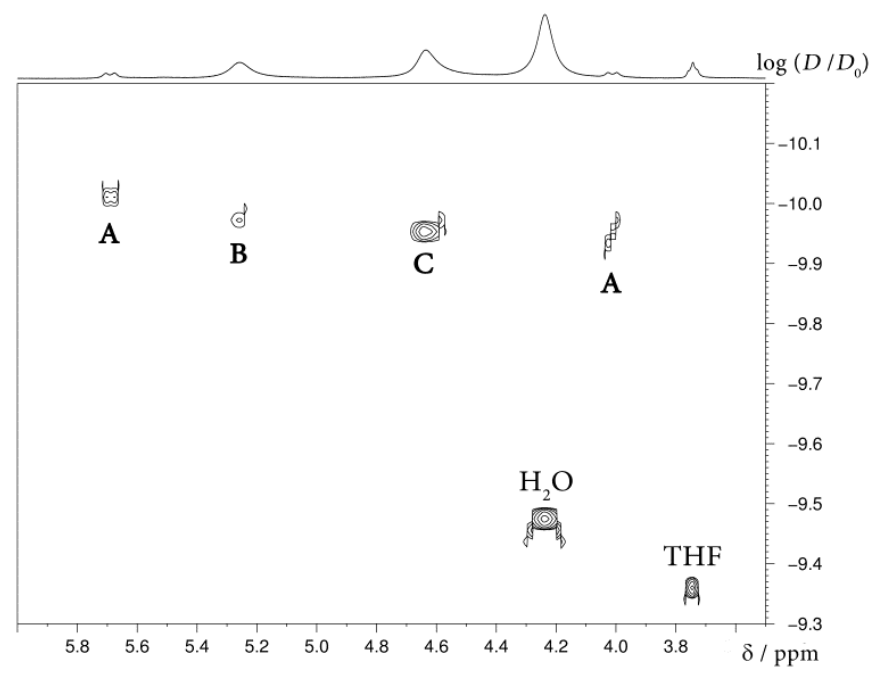

Figure 2-31: Excerpt from the ${ }^{1} \mathrm{H}$-DOSY spectrum of $\left.\left[\mathrm{ZnBr}(\mathrm{DMF})\left(\left(\mathrm{Me}_{2} \mathrm{NCH}_{2}\right)_{2} \mathrm{Acr}\right)\right)\right]^{+}$in $\mathrm{DMF}-d_{7}$ at $-30^{\circ} \mathrm{C}$ after the addition of 10 eq. $\mathrm{NaBr}$. The three methylene-resonances display different diffusion coefficients which further underlines the formation of three species $(\mathbf{A}, \mathbf{B}$ and $\mathbf{C}) .^{\mathrm{a}}\left(D_{0}=1 \mathrm{~m}^{2} / \mathrm{s}\right)$

Even though the diffusion coefficients of the different methylene signals vary only slightly, they match the tendency for size of the proposed solution state structures. Since the van-der-Waals volume of bromine is smaller than of DMF $\left(V_{\mathrm{vdW}}(\mathrm{Br})=2.74 \cdot 10^{-29} \mathrm{~m}^{3} ; V_{\mathrm{vdW}}(\mathrm{DMF})=13.1 \cdot 10^{-29} \mathrm{~m}^{3}\right)$ the largest species should be $\left.\left[\mathrm{Zn}(\mathrm{DMF})_{2}\left(\left(\mathrm{Me}_{2} \mathrm{NCH}_{2}\right)_{2} \mathrm{Acr}\right)\right)\right]^{2+}$ which is in this case aggregate $\mathbf{A}$, whereas $\left.\left[\mathrm{ZnBr}(\mathrm{DMF})\left(\left(\mathrm{Me}_{2} \mathrm{NCH}_{2}\right)_{2} \mathrm{Acr}\right)\right)\right]^{+}$and $\left.\left[\mathrm{ZnBr}_{2}\left(\left(\mathrm{Me}_{2} \mathrm{NCH}_{2}\right)_{2} \mathrm{Acr}\right)\right)\right]$ would be aggregates $\mathbf{B}$ and $\mathbf{C}$, respectively. Unfortunately, no ECCs have been established for DMF- $d_{7}$ and therefore these findings cannot be used for $M W$ predictions. However, the diffusion coefficients and the behavior of the signals after the addition of $\mathrm{NaBr}$ corroborate the assumptions.

\subsubsection{Solvation of a Heterocyclic Substituted Methanide in $C_{6} D_{6}$}

Another important aim of our group is the stabilization of low oxidation states of metals. To further foster this goal, different bisheterocyclomethanides which offer the potential to shield coordinated low-valent metal cations as well as some corresponding Group 13 metal complexes have been synthesized and characterized recently. ${ }^{[153]}$ These studies show that such ligand systems hold great potential for metal coordination and therefore offer promising opportunities for investigation. That in mind, Köhne synthesized the bis-(4,6-tertbutyl-benzoxazol-2-yl)-methanide ligand (4,6- $\left.t \mathrm{Bu}-\mathrm{NCOC}_{6} \mathrm{H}_{2}\right)_{2} \mathrm{CH}_{2}$; Scheme 2-7).

\footnotetext{
a Since a high content of water was in the samples, we also tried varying this water content. This did not result in significant changes of any signals and therefore its influence can be dismissed. Elevated diffusion coefficient of water is possibly a result of hydrogen bonding.
} 

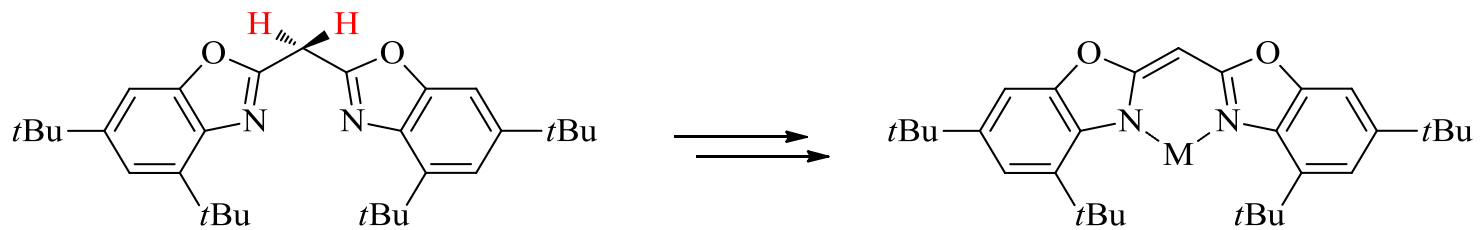

$$
\mathrm{M}=\mathrm{Li}^{+}, \mathrm{K}^{+}, \mathrm{Mg}^{2+}
$$

Scheme 2-7: Structure of bis-(4,6-tertbutyl-benzoxazol-2-yl)-methanide and its deprotonated and metalated form.

The ligand backbone can be deprotonated which enables the coordination of different metal cations by the two endocyclic nitrogen donor atoms, thus forming a six-membered ring system. This deprotonation is a good indication of a successful synthesis, as the resonance of the methanide $(\delta=$ $4.19 \mathrm{ppm}$; marked red in Scheme 2-7) is shifted significantly in the ${ }^{1} \mathrm{H}$ NMR spectra of the metal complexes (e.g. for $\left[\mathrm{Li}\left(4,6-t \mathrm{Bu}-\mathrm{NCOC}_{6} \mathrm{H}_{2}\right)_{2} \mathrm{CH}\right] \delta=5.59 \mathrm{ppm}$; for $\left[\mathrm{MgCl}_{2}(4,6-t \mathrm{Bu}-\right.$ $\left.\left.\mathrm{NCOC}_{6} \mathrm{H}_{2}\right)_{2} \mathrm{CH}\right]^{-} \delta=5.65 \mathrm{ppm}$ ), while its integral halves. Furthermore, all other ligand signals (two doublets of the benzoxazole at $\delta=7.39$ and $7.20 \mathrm{ppm}$ as well as two singlets of the $t \mathrm{Bu}$-groups at $\delta=$ 1.67 and $1.23 \mathrm{ppm}$ ) shift slightly as well after metalation.

To fully characterize the resulting organometallic reagents $\left(\left[\mathrm{Li}\left(4,6-t \mathrm{Bu}-\mathrm{NCOC}{ }_{6} \mathrm{H}_{2}\right)_{2} \mathrm{CH}\right]\right.$, $[\mathrm{K}(4,6-$ $\left.\left.t \mathrm{Bu}-\mathrm{NCOC}_{6} \mathrm{H}_{2}\right)_{2} \mathrm{CH}\right],\left[\mathrm{MgCl}_{2}\left(4,6-t \mathrm{Bu}-\mathrm{NCOC}_{6} \mathrm{H}_{2}\right)_{2} \mathrm{CH}\right]^{-}$and $\left.\left[\mathrm{MgBr}_{2}\left(4,6-t \mathrm{Bu}-\mathrm{NCOC}_{6} \mathrm{H}_{2}\right)_{2} \mathrm{CH}\right]^{-}\right)$and understand their aggregation behavior, ECC-DOSY $M W$-estimations have been performed in cooperation with Köhne. ${ }^{a}$ All compounds have been analyzed in $\mathrm{C}_{6} \mathrm{D}_{6}$ with the internal reference TMB. Also, there were traces of THF remaining from synthesis in all samples. $M W_{\text {det }}$ have been estimated with merged, DSE and ED ECCs. The ED ECC is in this instance helpful, as especially the protonated ligand can be described as flat rather than spherical. $M W_{\text {det }}$ were then compared to monomeric aggregates featuring varying solvation, i.e. $\left[\mathrm{M}\left(4,6-t \mathrm{Bu}-\mathrm{NCOC}_{6} \mathrm{H}_{2}\right)_{2} \mathrm{CH}(\mathrm{THF})_{\mathrm{x}}\right]$ with $\mathrm{x}=$ $0-2 ; \mathrm{M}=\mathrm{Li}, \mathrm{K}, \mathrm{MgCl}_{2-\mathrm{x}} ; \mathrm{MgBr}_{2-\mathrm{x}}$. The protonated ligand has also been assumed to be monomeric, i.e. $\left(4,6-t \mathrm{Bu}-\mathrm{NCOC}_{6} \mathrm{H}_{2}\right)_{2} \mathrm{CH}_{2}$. Results are given in Table 2-20 for the most likely species (the complete set of data is given in the appendix: Table 5-57 ((4,6- $\left.\left.t \mathrm{Bu}-\mathrm{NCOC}_{6} \mathrm{H}_{2}\right)_{2} \mathrm{CH}_{2}\right)$; Table 5-58 ([Li(4,6- $\left.\left.\left.t \mathrm{Bu}-\mathrm{NCOC}_{6} \mathrm{H}_{2}\right)_{2} \mathrm{CH}\right]\right)$; Table 5-59 ([K(4,6-tBu-NCOC $\left.\left.\left.\mathrm{H}_{2}\right)_{2} \mathrm{CH}\right]\right)$; Table 5-60 $\left(\left[\mathrm{MgCl}_{2}(4,6-\right.\right.$ $\left.\left.\left.t \mathrm{Bu}-\mathrm{NCOC}_{6} \mathrm{H}_{2}\right)_{2} \mathrm{CH}\right]^{-}\right)$; Table 5-61 $\left.\left(\left[\mathrm{MgBr}_{2}\left(4,6-t \mathrm{Bu}-\mathrm{NCOC}_{6} \mathrm{H}_{2}\right)_{2} \mathrm{CH}\right]^{-}\right)\right)$.

Note that the ECCs were only prepared for molecules with MWs of up to $600 \mathrm{~g} / \mathrm{mol}$ and that some $M W_{\text {det }}$ exceed this range. While it is straightforward that for these methanides always monomeric aggregation is preferred, the exact solvation state is in some cases not as easily determinable. For the alkali metal complexes everything points towards a threefold coordination incorporating a single THF molecule, i.e. $\left[\mathrm{M}\left(4,6-t \mathrm{Bu}-\mathrm{NCOC}_{6} \mathrm{H}_{2}\right)_{2} \mathrm{CH}(\mathrm{THF})\right]$ with $\mathrm{M}=\mathrm{Li}, \mathrm{K}$. $[\mathrm{K}(4,6-t \mathrm{Bu}-$ $\left.\mathrm{NCOC}_{6} \mathrm{H}_{2}\right)_{2} \mathrm{CH}$ ] would also be a possibility, but is more unlikely. The preferred coordination

\footnotetext{
${ }^{a}$ As this is an ongoing investigation for the Ph.D. thesis of Köhne, further information regarding syntheses and characterizations (e.g. individual NMR assignments and crystal structures) will not be given herein. Findings will be mentioned, if they help elucidate the solvation or aggregation state of the different molecules. ${ }^{\mathrm{b}}$ Dimeric aggregates can be excluded.
} 
number of four for the alkali metals is either way not achieved, possibly due to the sterical hindrance provided by the ligand.

Table 2-20: ECC ${ }^{\mathrm{C} 6 \mathrm{D} 6}$ (DSE, ED and merged) were used to predict $M W_{\text {det }}$ of $\left(4,6-t \mathrm{Bu}-\mathrm{NCOC}_{6} \mathrm{H}_{2}\right)_{2} \mathrm{CH}_{2}$, $[\mathrm{Li}(4,6-t \mathrm{Bu}-$ $\left.\left.\mathrm{NCOC}_{6} \mathrm{H}_{2}\right)_{2} \mathrm{CH}\right]$, $\left[\mathrm{K}\left(4,6-t \mathrm{Bu}-\mathrm{NCOC}_{6} \mathrm{H}_{2}\right)_{2} \mathrm{CH}\right],\left[\mathrm{MgCl}_{2}\left(4,6-t \mathrm{Bu}-\mathrm{NCOC}_{6} \mathrm{H}_{2}\right)_{2} \mathrm{CH}\right]$ and $\left[\mathrm{MgBr}_{2}\left(4,6-t \mathrm{Bu}-\mathrm{NCOC}_{6} \mathrm{H}_{2}\right)_{2} \mathrm{CH}\right]$ in $\mathrm{C}_{6} \mathrm{D}_{6}$. The accuracy of the $\mathrm{ECC}^{\mathrm{C} 6 \mathrm{D} 6}$ (DSE) is in the range of $M W_{\mathrm{dif}}= \pm 8 \%$, of the $\mathrm{ECC}^{\mathrm{C} 6 \mathrm{D} 6}$ (ED) in the range of $M W_{\mathrm{dif}}= \pm 6 \%$ and of the $\mathrm{ECC}^{\mathrm{C} 6 \mathrm{D} 6}$ (merged) in the range of $M W_{\mathrm{dif}}= \pm 19 \%$. Theoretical errors, as discussed in section 2.1.3, are given as standard deviations for all estimated $M W$ s. All results in this table are from measurements at $25^{\circ} \mathrm{C} . M W_{\text {dif }}$ is given for most likely monomeric aggregates for either DSE or ED ECCs.

\begin{tabular}{|c|c|c|c|c|c|}
\hline Possible aggregate(s) & $\begin{array}{c}M W_{\text {calc }} \\
{[\mathrm{g} / \mathrm{mol}]}\end{array}$ & $\begin{array}{c}M W_{\text {det }} \\
\text { (merged) }[\mathrm{g} / \mathrm{mol}]\end{array}$ & $\begin{array}{c}M W_{\mathrm{det}} \\
(\mathrm{DSE})[\mathrm{g} / \mathrm{mol}]\end{array}$ & $\begin{array}{c}M W_{\mathrm{det}} \\
(\mathrm{ED})[\mathrm{g} / \mathrm{mol}]\end{array}$ & $\begin{array}{c}M W_{\mathrm{dif}} \\
{[\%]}\end{array}$ \\
\hline$\left(4,6-t \mathrm{Bu}-\mathrm{NCOC}_{6} \mathrm{H}_{2}\right)_{2} \mathrm{CH}_{2}$ & 475 & $607 \pm 51$ & $545 \pm 30$ & $483 \pm 61$ & $-2(\mathrm{ED})$ \\
\hline$\left[\mathrm{Li}\left(4,6-\mathrm{tBu}-\mathrm{NCOC}_{6} \mathrm{H}_{2}\right)_{2} \mathrm{CH}(\mathrm{THF})\right]$ & 553 & $643 \pm 55$ & $575 \pm 32$ & $503 \pm 64$ & -4 (DSE) \\
\hline$\left[\mathrm{K}\left(4,6-t \mathrm{Bu}-\mathrm{NCOC}_{6} \mathrm{H}_{2}\right)_{2} \mathrm{CH}\right]$ & 513 & $620 \pm 52$ & $556 \pm 31$ & $490 \pm 62$ & -8 (DSE) \\
\hline$\left[\mathrm{K}\left(4,6-t \mathrm{Bu}-\mathrm{NCOC}{ }_{6} \mathrm{H}_{2}\right)_{2} \mathrm{CH}(\mathrm{THF})\right]$ & 585 & & & & 5 (DSE) \\
\hline$\left[\mathrm{MgCl}_{2}\left(4,6-t \mathrm{Bu}-\mathrm{NCOC}_{6} \mathrm{H}_{2}\right)_{2} \mathrm{CH}\right]^{-}$ & 569 & $685 \pm 59$ & $609 \pm 34$ & $527 \pm 67$ & -7 (DSE) \\
\hline$\left[\mathrm{MgCl}\left(4,6-t \mathrm{Bu}-\mathrm{NCOC}{ }_{6} \mathrm{H}_{2}\right)_{2} \mathrm{CH}(\mathrm{THF})\right]$ & 606 & & & & -1 (DSE) \\
\hline$\left[\mathrm{Mg}\left(4,6-t \mathrm{Bu}-\mathrm{NCOC}{ }_{6} \mathrm{H}_{2}\right)_{2} \mathrm{CH}(\mathrm{THF})_{2}\right]^{+}$ & 642 & & & & 5 (DSE) \\
\hline$\left[\mathrm{MgBr}\left(4,6-t \mathrm{Bu}-\mathrm{NCOC}{ }_{6} \mathrm{H}_{2}\right)_{2} \mathrm{CH}(\mathrm{THF})\right]$ & 650 & $715 \pm 62$ & $634 \pm 36$ & $544 \pm 70$ & 3 (DSE) \\
\hline$\left[\mathrm{Mg}\left(4,6-t \mathrm{Bu}-\mathrm{NCOC}{ }_{6} \mathrm{H}_{2}\right)_{2} \mathrm{CH}(\mathrm{THF})_{2}\right]^{+}$ & 642 & & & & 1 (DSE) \\
\hline
\end{tabular}

For the magnesium compounds a coordination number of four was postulated leading to aggregates like $\left[\mathrm{MgHal}_{2-\mathrm{x}}\left(4,6-t \mathrm{Bu}-\mathrm{NCOC}_{6} \mathrm{H}_{2}\right)_{2} \mathrm{CH}(\mathrm{THF})_{\mathrm{x}}\right]$ with $\mathrm{Hal}=\mathrm{Cl}$, Br. However, the $\left[\mathrm{MgBr}_{2}\left(4,6-t \mathrm{Bu}-\mathrm{NCOC}_{6} \mathrm{H}_{2}\right)_{2} \mathrm{CH}\right]^{-}$aggregate can be disregarded, since it displays elevated $M D_{\mathrm{w}}$. All other possible aggregates shown in Table 2-20 do not (the complete set of $M D_{\mathrm{W}}$ calculations is given in the appendix: Table 5-63). Other than that, all listed magnesium aggregates seem based on the estimated $M W$ s theoretically possible.

To elucidate the exact solvation of $\left[\mathrm{MgCl}_{2}\left(4,6-t \mathrm{Bu}-\mathrm{NCOC}_{6} \mathrm{H}_{2}\right)_{2} \mathrm{CH}\right]^{-}$, the normalized diffusion coefficient of THF in the measured sample was determined $\left(\log \left(D_{\mathrm{x}, \mathrm{norm}}\right)=-8.9318\right)$ and compared with the normalized diffusion coefficient of separately measured free THF $\left(\log \left(D_{\mathrm{x}, \mathrm{norm}}\right)=-8.6371\right)$ and the $\left[\mathrm{MgCl}_{2}\left(4,6-t \mathrm{Bu}-\mathrm{NCOC} \mathrm{H}_{2}\right)_{2} \mathrm{CH}(\mathrm{THF})_{\mathrm{x}}\right]^{-}$aggregate $\left(\log \left(D_{\mathrm{x}, \mathrm{norm}}\right)=-9.2020\right)$. Integration showed that approximately 1.25 eq. THF were in the sample altogether. Out of this amount of THF, about $50 \%$ coordinated based on the elevated diffusion coefficients compared to the diffusion coefficient of a pure THF solution and to the diffusion coefficient of the metal complex. Another sample was prepared with approximately 2.75 eq. THF which showed that about $55 \%$ aided in the solvation of the metal complex. In both cases the number of coordinating THF molecules is between 0 and 2, as proposed by the ECC calculations. It seems to be a defined equilibrium, wherein possibly a chloride is exchanged by THF, whereas always about $50 \%$ of the available THF is involved. In the future, it might be interesting to further vary the THF concentration and see if a saturation is achieved after a certain amount. Also, the addition of e.g. $\mathrm{NaCl}$ may help direct the equilibrium towards the chlorinated species and thus provide further insights. Similar experiments can be envisioned for the brominated reagent. 
Lastly, the stabilization of low oxidation states was the aim of the synthesis of these structures. Therefore, Köhne tried reducing the $\left[\mathrm{MgCl}_{2}\left(4,6-t \mathrm{Bu}-\mathrm{NCOC}_{6} \mathrm{H}_{2}\right)_{2} \mathrm{CH}\right]^{-}$compound. The resulting reaction mixture was also investigated with ECC-DOSY and showed elevated diffusion coefficients for the resulting reagent, whereas other spectroscopic data (chemical shifts) did not vary significantly. The estimated $M W \mathrm{~s}$ were $993 \pm 90 \mathrm{~g} / \mathrm{mol}$ for the merged ECC and $857 \pm 50 \mathrm{~g} / \mathrm{mol}$ for the DSE ECC. Both exceed $600 \mathrm{~g} / \mathrm{mol}$ and it was therefore questionable how accurate an ECC- $M W$ estimation could be. Köhne also investigated the molecule by the LIFDI-MS technique (LIFDI = liquid injection field desorption ionization) and could show that the aggregate is not the expected reduced species, but rather $\left[\mathrm{Mg}\left(\left(4,6-t \mathrm{Bu}-\mathrm{NCOC}_{6} \mathrm{H}_{2}\right)_{2} \mathrm{CH}\right)_{2}\right]$ (see Scheme 2-8) with a $M W_{\text {calc }}$ of $972 \mathrm{~g} / \mathrm{mol}$.

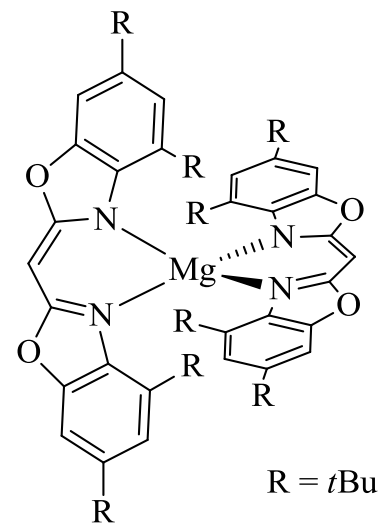

Scheme 2-8: Structure of bis-(bis-(4,6-tertbutyl-benzoxazol-2-yl)-methanide)-magnesium.

This aggregate corresponds well with the $M W$ estimated by the merged ECC. While the synthesis of this aggregate was not the envisioned reduced species, it still was an important result to show the limits of the shape-optimized ECCs. These do not work, when the $M W$ range of the model compounds is exceeded. However, the merged ECC seems to be reliable. Note that this is only a single result and the usability of the ECC $M W$-prediction for bigger aggregates needs to be investigated further to make a conclusion. 


\section{SUMMARY \& OUTLOOK}

The focus of this thesis has been the development and application of a high-resolution NMR spectroscopic diffusion-based technique, i.e. external calibration curve DOSY (ECC-DOSY), for molecular weight $(M W)$ estimation of solutes. In the three parts of this thesis, it was not only possible to augment this technique by many important features, but also to explain and alleviate some of the pitfalls of the methodology as well as to unambiguously solve solution state structures of different organometallic reagents and precursor molecules.

In the first part of this thesis, the applicability of ECC-DOSY was severely improved by the addition of new ECCs for seven commonly used deuterated solvents, namely chloroform- $d_{1}$, dichloromethane- $d_{2}$, benzene- $d_{6}$, dimethylsulfoxide- $d_{6}$, cyclohexane- $d_{12}$, acetonitrile- $d_{3}$ and methanol- $d_{4}$ to enable $M W$ predictions. Merged as well as three semi-empirically shape-optimized ECCs could be established for each solvent, utilizing the normalized diffusion coefficients of up to 31 model compounds with a $M W$-range of 70 to $600 \mathrm{~g} / \mathrm{mol}$. These normalized diffusion coefficients are the first step towards solvent-dependent normalized diffusion coefficient databases. The need for such databases or at least for the increase of the overall reference count could be shown in this work, as the misconception of a single unsuited reference has led for some solvents (DMSO and cyclohexane) to reduced fit qualities of their corresponding ECCs. Fortunately, this model compound $\left(\mathrm{Si}(\mathrm{OMe})_{4}\right)$ could be identified after careful evaluation. Furthermore, not only the diversification of the overall references has to be improved, but also the empirical categorization process. Hence, a more theoretical approach to categorization was tested utilizing the calculation of principal moments of inertia. While this molecular property seems feasible to distinguish very spherical and linear molecules, the minute changes in molecular shape that most of the herein-used model compounds exhibited cannot be described reasonably well.

However, overall ECCs are shown to be very accurate even without any categorization. Empirical deviations of predicted $M W_{\text {det }}$ compared to theoretical $M W_{\text {calc }}$ did not exceed a maximum of $29 \%$ for the merged ECCs. Average deviations (and deviations of shape-optimized ECCs) are in most cases much better. To further underline the method's accuracy, theoretical error calculations were performed. In most cases, these corresponded well with the empirical deviations, however, in a few cases a possible underestimation of errors might be conceivable, if just the empirical deviations were considered. Additionally, the influence of molecular densities $\left(M D_{\mathrm{W}}\right)$ was discussed and in cooperation with Kreyenschmidt a method was developed which might prevent $M D_{\mathrm{W}}$-related errors in the future, as it entails the application of a $M D_{\mathrm{W}}$-based correctional factor $X_{\text {cor }}$ to normalized diffusion coefficients. ${ }^{[124]}$ However, this approach has one setback, the need to predict the solution state structure of a solute a priori. All these improvements to the methodology are or will be implemented in a software application that hopefully widens the audience of ECC-DOSY. They are also shown schematically in Figure 3-1. 


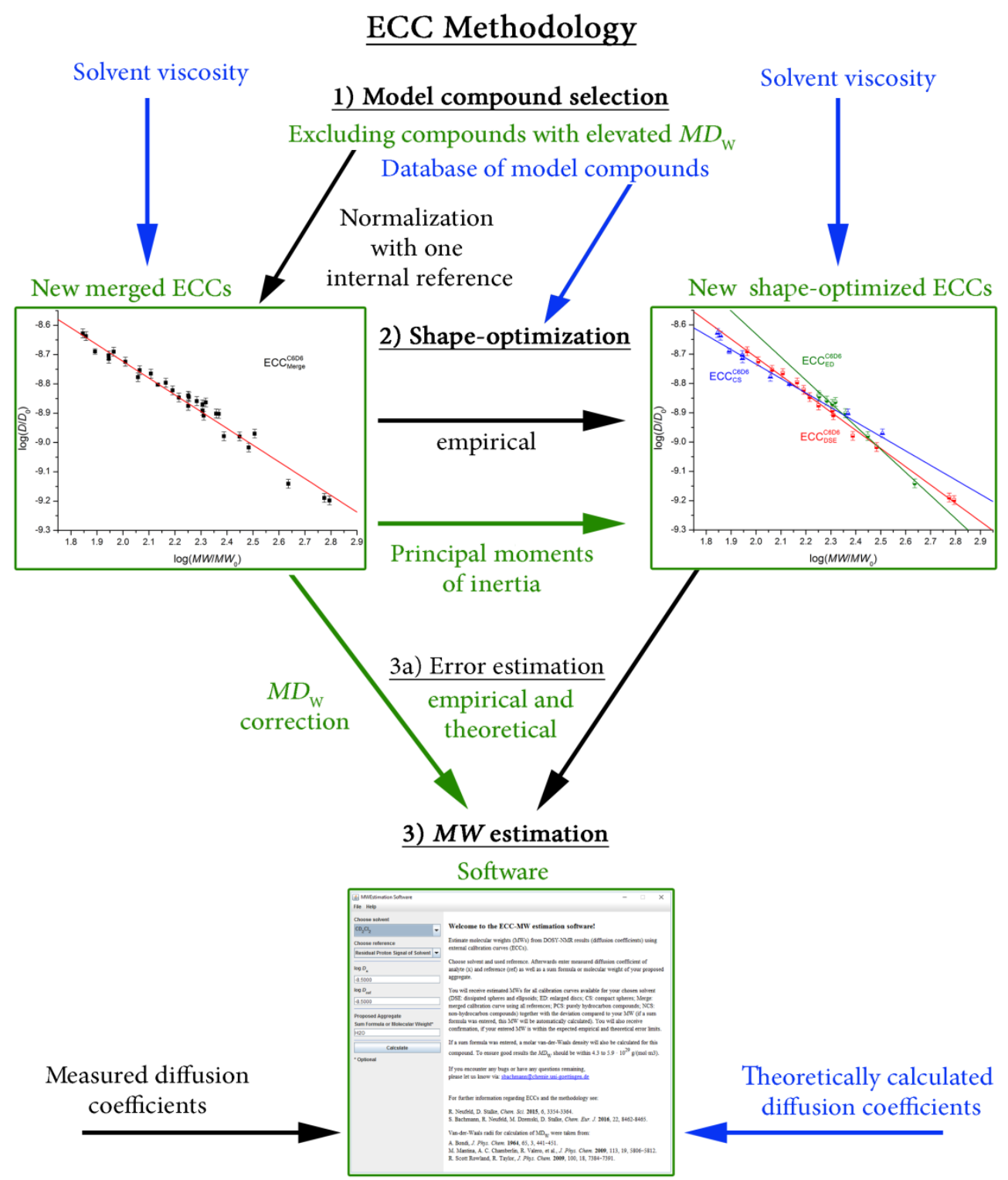

Figure 3-1: Schematic representation of the ECC-DOSY-MW estimation process. Highlighted green are improvements and implementations of this work, whereas blue objects are proposed additions for the future.

As of now, only measured diffusion coefficients are considered in the $M W$ prediction, however, as calculation prowess improves, theoretically calculated diffusion coefficients might end up being an important addition. Furthermore, a so-called universal calibration curve (UCC) has surfaced very recently in the polymer community which incorporates solvent viscosity for $M W$ estimations and hence eliminates the need to distinguish solvent environments. ${ }^{[154]}$ However, this method seemed to be less accurate for small molecules. Still, if solvent viscosity can be implemented into the ECC methodology in the future, it could streamline the entire process and with the data gathered in this work, it might also be possible to detect aberrations beyond viscosity-related effects. There are such aberrations, else all ECCs with the same references should not vary in their $\alpha$ parameters.

In the second part of this thesis, the applicability of ECC-DOSY was demonstrated on a widelyused group of organometallic precursors, i.e. alkali metal cyclopentadienides (CpMs, with $\mathrm{M}=\mathrm{Li}$, $\mathrm{Na}, \mathrm{K}, \mathrm{Rb}$ and $\mathrm{Cs}$ ). These reagents were characterized in THF and DMSO. CpLi was also 
investigated in ammonia solution. While in the solid state most of these reagents form polymeric chains, even in some cases with donating solvents (e.g. [CpNa(TMEDA) $]_{\infty}^{[52]}$ ), the results gathered in this thesis suggest that in THF they are fully disaggregated into monomers. The same observation was made for some methylated cyclopentadienide derivatives ( $\mathrm{MeCpLi}, \mathrm{Cp}^{\star} \mathrm{K}$ and $\mathrm{Cp}^{\star} \mathrm{Cs}$ ). Even at different temperatures these species stay intact. This was shown for CpLi which was investigated for a temperature range from $50{ }^{\circ} \mathrm{C}$ to $-100^{\circ} \mathrm{C}$. The only exception was $\mathrm{CpCs}$ which displayed oligomeric aggregation in THF, forming an aggregate of $M W_{\text {det }}>1500 \mathrm{~g} / \mathrm{mol}$ which goes far beyond the $M W$ range of measured model compounds. With ECC estimations the degree of solvation could also be determined and the solution state structures of all CpMs depicted in Figure 3-2 show the best accordance of theoretical and experimental MWs.
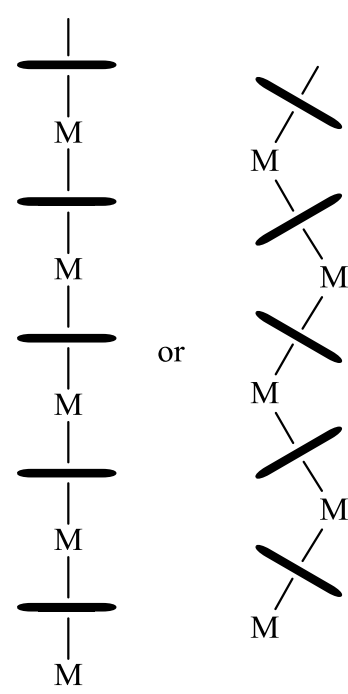

$\mathrm{M}=\mathrm{Li}, \mathrm{Na}, \mathrm{K}, \mathrm{Rb}, \mathrm{Cs}$

solid state
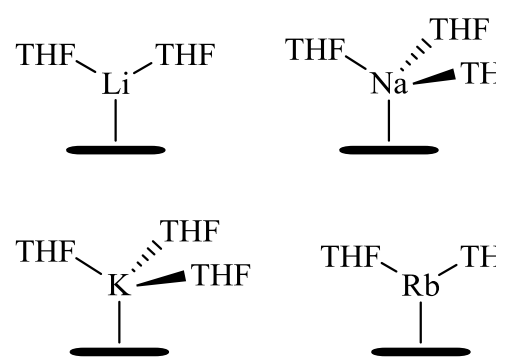

THF

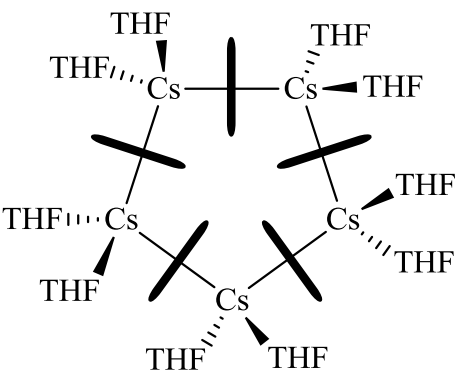

solution

Figure 3-2: Disaggregation of polymeric alkali metal cyclopentadienides after solvation and the corresponding solution state structures in THF- $d_{8}$ proposed after investigation by ECC-DOSY.

While CpMs formed solely contact ion pairs (CIPs) in THF, which could also be underlined by the comparison of diffusion coefficients gathered from ${ }^{1} \mathrm{H}$ - and ${ }^{7} \mathrm{Li}-\mathrm{DOSY}$ experiments for $\mathrm{CpLi}$, inDMSO and ammonia, where they seem to form solvent-separated ion pairs (SSIPs). Unfortunately, $M W$ estimations did not result in reasonable structures; possibly due to dynamic exchange processes that average diffusion coefficients. However, through ${ }^{7} \mathrm{Li},{ }^{1} \mathrm{H}-\mathrm{HOESY}$ and diffusion coefficient comparison the formation of SSIPs can at least for CpLi be confirmed. For the other CpMs a comparison of normalized diffusion coefficients showed the same behavior as observed for $\mathrm{CpLi}$, therefore SSIPs are also most likely. Proceeding, it might be a good idea to take a closer look at the methylated derivatives, since it could already be shown that $\mathrm{Cp}^{*} \mathrm{Cs}$ behaves differently to CpCs. Also, other solvent environments may present an interesting opportunity for investigation. 
In the third and final chapter, NMR spectroscopy in general and diffusion-based approaches were used to solve ambiguities about solution state structures of different organometallic compounds or their precursors provided by collaborators (see Scheme 3-1).

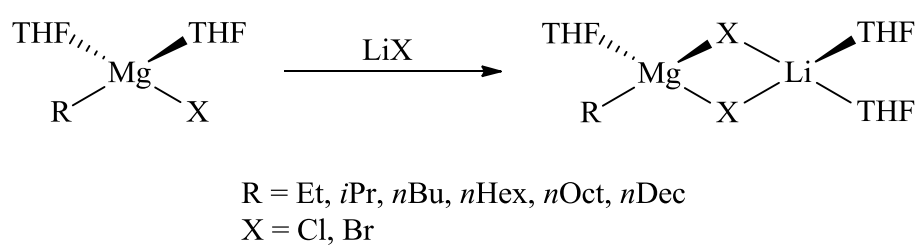

Alkyl Grignard reagents as well as "turbo" analogues<smiles></smiles>

$N$-Heterocyclic carbene

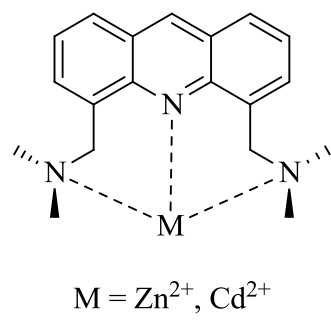

Acridine-based molecular sensor

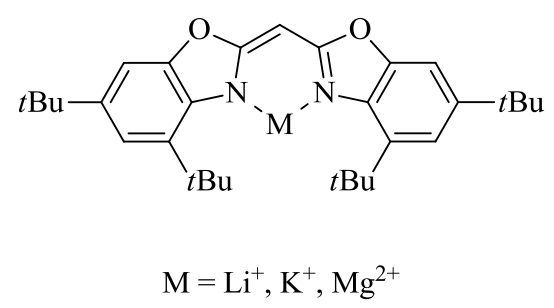

Heterocyclic substituted methanide

Scheme 3-1: Illustration of the different research areas discussed in the final part of this thesis.

Firstly, alkyl Grignard systems were shown to form monomers in THF solution, i.e. [RMgX $\left.(\mathrm{THF})_{2}\right]$ with $\mathrm{R}=\mathrm{Et}, i \mathrm{Pr}, n \mathrm{Bu}, n \mathrm{Hex}, n \mathrm{Oct}$ and $n \mathrm{Dec}$ and $\mathrm{X}=\mathrm{Cl}$ (for $\mathrm{R}=n \mathrm{Bu}, \mathrm{X}=\mathrm{Cl}, \mathrm{Br}$ ). The clear preference of this solvation state could be shown through the establishment of further shape-optimized ECCs specialized for linear or rod-like molecules. Important to note is in this regard the ability to differentiate suitable model compounds by principle moment of inertia calculations. Furthermore, the influence of metal halides on these Grignard systems was elucidated exemplary for $[n \mathrm{BuMgX} \cdot \mathrm{LiX}](\mathrm{X}=\mathrm{Cl}, \mathrm{Br})$. It can be concluded that the addition of LiX leads to the formation of ate complexes at least to some degree which Knochel et al. proposed previously. ${ }^{[142]}$ In the investigation of these conventional alkyl Grignard reagents, the importance of the combination of different analytical techniques could also be emphasized, e.g. by comparison of findings from NMR spectroscopy to ESI-MS (see Figure 3-3). 


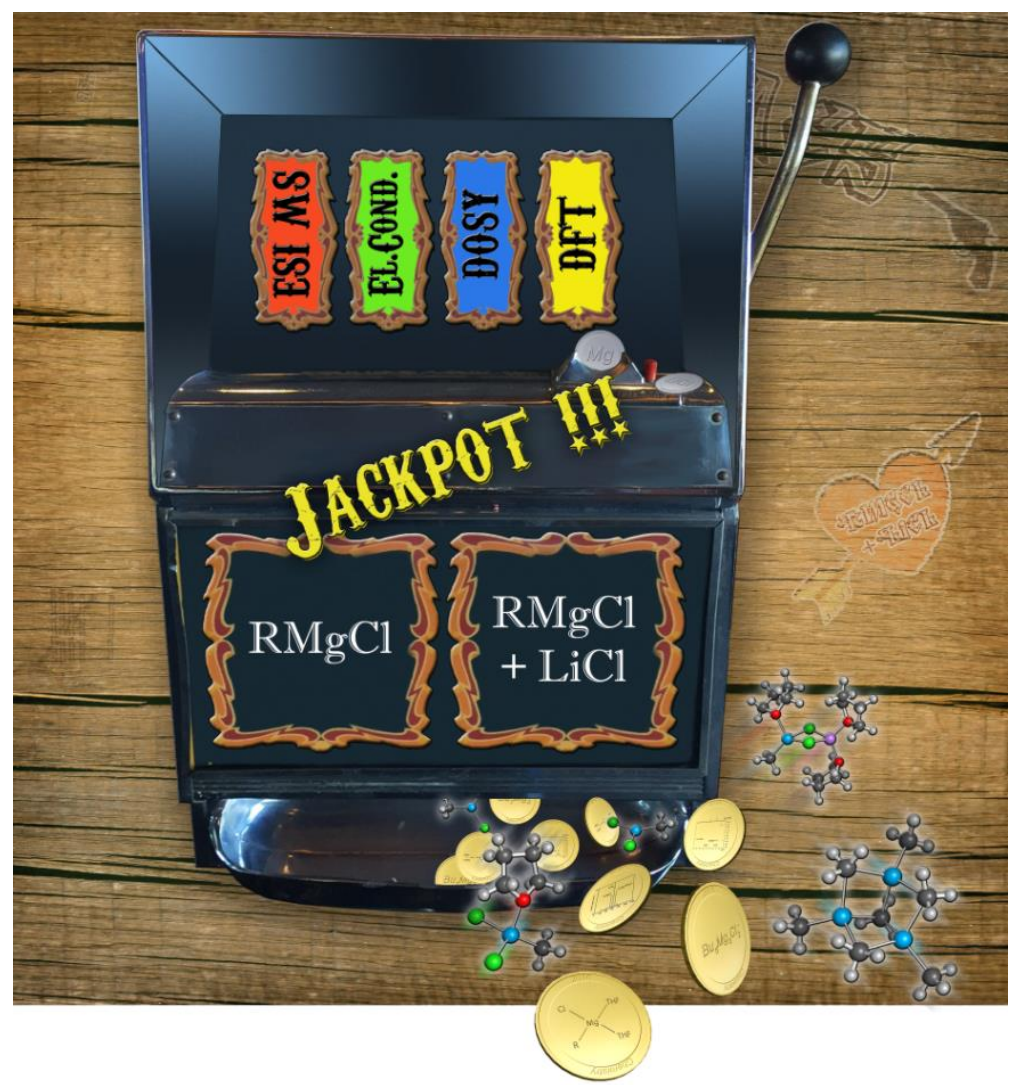

Figure 3-3: Frontispiece associated to "Association and Dissociation of Grignard Reagents RMgCl and Their Turbo Variant $\mathrm{RMgCl} \cdot \mathrm{LiCl}$ ” published in Chem. Eur. J. 2016, 22, 7752-7762. ${ }^{[3]}$

Additionally, solvent- and anion coordination were investigated for a multitude of different reagents: Ranging from an $N$-heterocyclic carbene that displayed interactions with a wide range of anions over an acridine-based fluorophore and its metal complexes which could be investigated via NMR and whose $\mathrm{Zn}^{2+}$ metal complex displayed an anion-solvent exchange equilibrium to an heterocyclic substituted methanide that not only showed concentration dependent solvent coordination, but also an aggregate that exceeded the recommended $M W$ range and could therefore be used to rationalize the suitability of the ECCs for higher aggregates. 


\section{EXPERIMENTAL PART}

\subsection{Techniques and Experiments}

\subsubsection{Handling of Air- and Moisture-Sensitive Compounds}

All experiments were performed either in an inert gas atmosphere of purified dry argon with standard Schlenk techniques ${ }^{[9]}$ or in an argon glove box. Glassware was dried at $130{ }^{\circ} \mathrm{C}$, assembled hot and cooled down under vacuum. All solvents were dried over sodium-potassium alloy, sodium or potassium, distilled and degassed prior to use. Dicyclopentadiene was purchased from Fluka, thermally cracked and distilled directly before use at $170{ }^{\circ} \mathrm{C}$ and ambient pressure. The methylcyclopentadiene dimer was purchased from Sigma-Aldrich, also thermally cracked and distilled directly before use at $72{ }^{\circ} \mathrm{C}$ and ambient pressure. Other starting materials were either purchased commercially or synthesized according to known literature procedures and stored under inert atmosphere. $n$-Butyllithium was kindly donated by Rockwood Lithium and was filtered through Celite $^{\Theta}$ before use, along with the determination of the concentration. ${ }^{[155]}$ Deuterated solvents for NMR measurements of organometallic compounds were kept in an argon glove box over $3 \AA$ molecular sieves over a minimum of one week prior to use. THF- $d_{8}$ for alkyl Grignard measurements was dried over potassium and distilled prior to use.

\subsubsection{NMR Techniques and Experiments}

All NMR spectra were recorded either on a Bruker Avance III $400 \mathrm{MHz}$ spectrometer with a BBFO (broadband-observe) probe, z-gradient (maximum gradient strength of $57 \mathrm{G} / \mathrm{cm}$ ) and temperature unit or on a Bruker Ascend $400 \mathrm{MHz}$ spectrometer with a BBI (broadband-inverse) probe, zgradient (maximum gradient strength of $51 \mathrm{G} / \mathrm{cm}$ ) and temperature unit. All samples were prepared using $5 \mathrm{~mm}$ NMR tubes made of borosilicate glass. NMR spectra were measured with diluted solutions $(0.015-0.025 \mathrm{M})$ at ambient temperature, if not indicated otherwise. All relevant parameters regarding $1 \mathrm{D}$ and $2 \mathrm{D}$ experiments are given in Table 4-1.

Diffusion experiments $\left({ }^{1} \mathrm{H}\right.$ - and ${ }^{7} \mathrm{Li}$-DOSY $)$ were performed exclusively with the pulse program dstebpgp3 $\mathrm{s}^{[156]}$ (double-stimulated echo sequence in combination with bipolar gradient pulses) employing a linear gradient ramp incremented from 2 to $98 \%$ of the maximum gradient strength, 16 dummy scans (ds) and 16 scans on $32 \mathrm{~K}$ data points. This pulse program effectively reduces eddy-current distortions and dephasing due to convection which arises mostly at elevated temperatures due to heating that can lead to a temperature gradient along the main axis of the spectrometer. However, turbulent convection cannot be compensated by this pulse program. The relevant delays for ${ }^{1} \mathrm{H}$-diffusion experiments were $\Delta=0.1 \mathrm{~s}$ and $\delta=1.0-8.0 \mathrm{~ms}$, whereas for ${ }^{7} \mathrm{Li}$-DOSY measurements they were $\Delta=0.06-0.08 \mathrm{~s}$ and $\delta=2.0-8.5 \mathrm{~ms}$. To achieve ideal signal attenuation, $\Delta$ or $\delta$ were adjusted and 1D spectra (dstebpp3s1d) that only varied in their gradient 
strength (either $5 \%$ or $95 \%$ ) were recorded and compared. This was repeated until the signal attenuation was within a reasonable region. The delay for gradient recovery was $0.2 \mathrm{~ms}$ and the eddy-current delay $5 \mathrm{~ms}$. Furthermore, all diffusion measurements were performed without sample spinning.

Table 4-1: Parameters of all conducted 1D and 2D NMR experiments.

\begin{tabular}{llll}
\hline Experiment & Pulse program & Number of scans & Acquisition time [s] \\
\hline${ }^{1} \mathbf{H}$ & zg30 & $8-16$ & $4.9-5.1$ \\
${ }^{13} \mathbf{C}\left\{{ }^{1} \mathbf{H}\right\}$ & zgdc30 & $256-1024$ & $1.3-1.4$ \\
${ }^{6} \mathbf{L i}$ & zg30 & 128 & 1.1 \\
${ }^{7} \mathbf{L i}$ & zg30 & 128 & 1.1 \\
${ }^{23} \mathbf{N a}$ & zg & 16 & 19.3 \\
${ }^{133} \mathbf{C s}$ & zg & $64-256$ & $0.1-3.2$ \\
${ }^{1} \mathbf{H},{ }^{1} \mathbf{H}-\mathbf{C O S Y}$ & cosygpqf & $1(16 \mathrm{ds})$ & 0.2 \\
${ }^{1} \mathbf{H},{ }^{13} \mathbf{C}-\mathbf{H S Q C}$ & hsqcedgpph & $2(16 \mathrm{ds})$ & 0.2 \\
${ }^{1} \mathbf{H},{ }^{13} \mathbf{C}-\mathbf{H M B C}$ & hmbcgplpndqf & $2(16 \mathrm{ds})$ & 0.2 \\
${ }^{1} \mathbf{H},{ }^{15} \mathbf{N}-\mathbf{H M B C}$ & hmbcgpndqf & $2-40(16 \mathrm{ds})$ & $0.1-0.2$ \\
${ }^{1} \mathbf{H},{ }^{13} \mathbf{C d}-\mathbf{H M B C}$ & hmbcgplpndqf & 2 & 0.2 \\
${ }^{1} \mathbf{H},{ }^{7} \mathbf{L i}-\mathbf{H O E S Y}$ & hoesyph & $16-32(16 \mathrm{ds})$ & $0.5-2.6$ \\
& & & (mixing time $0.5-1.7 \mathrm{~s})$ \\
\hline
\end{tabular}

All spectra were processed with Topspin 3.1, MestReNova 6.02 or MestReNova 10.1. Diffusion coefficients from DOSY experiments were calculated with the T1/T2 software of Topspin 3.1 utilizing Gaussian fits. Chemical shifts $(\delta)$ are given in ppm relative to TMS, either using the residual solvent signals ${ }^{[157]}$ or tabulated frequency ratios as internal standards. Coupling constants $(J)$ are reported in $\mathrm{Hz}$ and standard abbreviations indicating multiplicity are used as follows: $\mathrm{s}=$ singlet, $\mathrm{d}=$ doublet, $\mathrm{t}=$ triplet, $\mathrm{m}=$ multiplet, $\mathrm{br}=$ broad. Combined abbreviations are derived from their components (e.g. $\mathrm{dd}=$ doublet of doublets).

\subsubsection{DOSY Sample Preparation and Evaluation}

Samples for DOSY NMR measurements were prepared by addition of equimolar amounts of one of the following internal references: 1,2,3,4-tetraphenylnaphthalene (TPhN), 2,2,3,3-tetramethylbutane (TMB) or adamantane (Adam). For all measurements, the diffusion coefficients of the analytes were normalized to the fixed diffusion coefficients of one of these references which are listed in Table 2-1 or given in the appendix. 


\subsubsection{Computation of Principal Moments of Inertia}

Principal moments of inertia of different model compounds were calculated using PMIFST 16a.IT.2016 ${ }^{[122]}$ (Principal Moments of Inertia From Molecular Structures) with force-field (MMFF94) optimized structures generated by Avogadro 1.0.3.

\subsubsection{Programming in Java}

An ECC- $M W$ estimation software was programmed to make the methodology of ECC-DOSY more accessible (for more information see section 2.1.5). The software is available for download at:

http://www.stalke.chemie.uni-goettingen.de/mwestimation/

To achieve a maximum system interoperability, the software was implemented in Oracle's objectoriented programming language Java. Java uses a so-called Java virtual machine (JVM) which can run bytecode and needs to be installed on the computer that launches the application. The bytecode itself was created by compiling the Java code with the help of the Java compiler. With the combination of JVM and the system independent bytecode it is possible to run the software on a wide range of operating systems, including, but not limited to, Windows ${ }^{\oplus}, \mathrm{MacOS}^{\oplus}$ and Linux. The source code of the program can be shared upon request. 


\subsection{Syntheses and Characterizations}

\subsubsection{Syntheses and Characterizations of $\mathrm{CpMs}(\mathrm{M}=\mathrm{Li}, \mathrm{Na}, \mathrm{K}, \mathrm{Rb}, \mathrm{Cs}$ )}

\subsubsection{Synthesis and Characterization of $\mathrm{CpLi}$}

Cyclopentadienyllithium (CpLi) was prepared following a preparation route similar to Olbrich's ${ }^{[54]}$ and Wagner' ${ }^{[158]}$. It was synthesized by lithiation of freshly distilled $\mathrm{CpH}(0.45 \mathrm{~mL}, 5.50 \mathrm{mmol}$, 1.16 eq.; from dicyclopentadiene see 4.1 .1$)$ with $n \operatorname{BuLi}(4.74 \mathrm{M}$ in hexane, $1.00 \mathrm{~mL}, 4.74 \mathrm{mmol}$, 1.00 eq.) at $0{ }^{\circ} \mathrm{C}$ in THF $(50 \mathrm{~mL})$. The colorless reaction mixture was stirred for $10 \mathrm{~h}$ and after evaporation of the solvent CpLi was received as a white powder $(0.34 \mathrm{~g}, 4.72 \mathrm{mmol}$, 99\%) and dried in vacuo. For NMR measurements in $\mathrm{NH}_{3}$, gaseous $\mathrm{NH}_{3}$ was introduced into the NMR tube for one minute at $-78^{\circ} \mathrm{C}$; afterwards $0.1 \mathrm{~mL}$ of toluene- $d_{8}$ was added for referencing and after sealing the NMR tube spectra were recorded at $25^{\circ} \mathrm{C}$.
Sum formula:
$\mathrm{C}_{5} \mathrm{H}_{5} \mathrm{Li}$
Molecular weight:
$72.04 \mathrm{~g} / \mathrm{mol}$

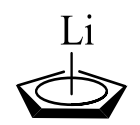

Dissolving the compound in different solvents (DMSO- $d_{6}, \mathrm{THF}-d_{8}, \mathrm{NH}_{3}$ ) resulted in different solution state structures and therefore different chemical shifts:

$\left(\right.$ THF- $\left.d_{8}\right)$ :

Proposed aggregate: $\quad\left[\mathrm{C}_{5} \mathrm{H}_{5} \mathrm{Li}(\mathrm{THF})_{2}\right]$

Sum formula: $\quad \mathrm{C}_{13} \mathrm{H}_{21} \mathrm{O}_{2} \mathrm{Li}$

Molecular weight: $\quad 216.25 \mathrm{~g} / \mathrm{mol}$

${ }^{1} \mathrm{H}$ NMR

(400.13 MHz, THF- $\left.\boldsymbol{d}_{8}\right)$ : $\quad \delta=5.69(\mathrm{~s}, 5 \mathrm{H}, 5 \mathrm{CH}) \mathrm{ppm}$.

${ }^{13} \mathrm{C}\left\{{ }^{1} \mathrm{H}\right\}$ NMR

(100.62 MHz, THF- $\left.\boldsymbol{d}_{\mathbf{8}}\right): \quad \delta=103.4(\mathrm{~s}, 5 \mathrm{C}, 5 \mathrm{CH}) \mathrm{ppm}$.

${ }^{7} \mathrm{Li}$ NMR

$\left(\mathbf{1 5 5 . 5 1} \mathrm{MHz}\right.$, THF- $\left.\boldsymbol{d}_{8}\right): \quad \delta=-7.75(\mathrm{~s}) \mathrm{ppm}$. 
(DMSO- $\left.d_{6}\right)$ :

\section{${ }^{1} \mathrm{H}$ NMR}

(400.13 MHz, DMSO- $\left.\boldsymbol{d}_{6}\right): \quad \delta=5.33(\mathrm{~s}, 5 \mathrm{H}, 5 \mathrm{CH}) \mathrm{ppm}$.

${ }^{13} \mathrm{C}\left\{{ }^{1} \mathrm{H}\right\}$ NMR

(100.62 MHz, DMSO- $\left.\boldsymbol{d}_{6}\right)$ : $\quad \delta=103.0(\mathrm{~s}, 5 \mathrm{C}, 5 \mathrm{CH}) \mathrm{ppm}$.

${ }^{7} \mathrm{Li}$ NMR

(155.51 MHz, DMSO- $\left.\boldsymbol{d}_{6}\right): \quad \delta=-1.07$ (s) ppm.

$\left(\mathrm{NH}_{3}\right)$ :

${ }^{1} \mathrm{H}$ NMR

(400.13 MHz, $\mathrm{NH}_{3}$ ):

$\delta=5.89(\mathrm{~s}, 5 \mathrm{H}, 5 \mathrm{CH}) \mathrm{ppm}$.

${ }^{13} \mathrm{C}\left\{{ }^{1} \mathrm{H}\right\}$ NMR

(100.62 MHz, $\left.\mathrm{NH}_{3}\right)$ : $\quad \delta=104.1(\mathrm{~s}, 5 \mathrm{C}, 5 \mathrm{CH}) \mathrm{ppm}$.

${ }^{7} \mathrm{Li}$ NMR

(155.51 MHz, $\left.\mathrm{NH}_{3}\right)$ : $\quad \delta=-0.59$ (s) $\mathrm{ppm}$. 


\subsubsection{Synthesis and Characterization of $\mathrm{CpNa}$}

Cyclopentadienylsodium (CpNa) was prepared following a preparation route similar to Olbrich's ${ }^{[54]}$. It was synthesized by addition of freshly distilled $\mathrm{CpH}(0.90 \mathrm{~mL}, 10.80 \mathrm{mmol}, 1.08 \mathrm{eq}$.; from dicyclopentadiene see 4.1.1) to a suspension of $\mathrm{NaH}\left(0.24 \mathrm{~g}, 10.00 \mathrm{mmol}, 1.00\right.$ eq.) in $\mathrm{Et}_{2} \mathrm{O}$ $(50 \mathrm{~mL})$ at ambient temperature. The reaction mixture was stirred for $24 \mathrm{~h}$ and turned light pink in the process. After filtration, cyclopentane $(10 \mathrm{~mL})$ and pentane $(20 \mathrm{~mL})$ were added to the filtrate and the product was crystallized at $-28^{\circ} \mathrm{C}$. The white crystals $(0.64 \mathrm{~g}, 7.27 \mathrm{mmol}, 73 \%)$ were filtered, washed with cold pentane and dried in vacuo.

$\begin{array}{ll}\text { Sum formula: } & \mathrm{C}_{5} \mathrm{H}_{5} \mathrm{Na} \\ \text { Molecular weight: } & 88.08 \mathrm{~g} / \mathrm{mol}\end{array}$

Dissolving the compound in different solvents (DMSO- $d_{6}$, THF- $d_{8}$ ) resulted in different solution state structures and therefore different chemical shifts:

(THF- $\left.d_{8}\right)$ :

Proposed aggregate: $\quad\left[\mathrm{C}_{5} \mathrm{H}_{5} \mathrm{Na}(\mathrm{THF})_{3}\right]$

Sum formula: $\quad \mathrm{C}_{17} \mathrm{H}_{29} \mathrm{O}_{3} \mathrm{Na}$

Molecular weight: $\quad 304.40 \mathrm{~g} / \mathrm{mol}$

${ }^{1} \mathrm{H}$ NMR

(400.13 MHz, THF- $\left.\boldsymbol{d}_{8}\right): \quad \delta=5.72(\mathrm{~s}, 5 \mathrm{H}, 5 \mathrm{CH}) \mathrm{ppm}$.

${ }^{13} \mathrm{C}\left\{{ }^{1} \mathrm{H}\right\}$ NMR

(100.62 MHz, THF- $\left.\boldsymbol{d}_{\mathbf{8}}\right): \quad \delta=103.3(\mathrm{~s}, 5 \mathrm{C}, 5 \mathrm{CH}) \mathrm{ppm}$.

${ }^{23} \mathrm{Na}$ NMR

$\left(\mathbf{1 0 5 . 8 4} \mathrm{MHz}\right.$, THF- $\left.\boldsymbol{d}_{\mathbf{8}}\right): \quad \delta=-28$ to $-31\left(\mathrm{~s}_{\mathrm{br}}\right) \mathrm{ppm}$.

(DMSO- $\left.d_{6}\right)$ :

${ }^{1} \mathrm{H}$ NMR

(400.13 MHz, DMSO-d $\left.\boldsymbol{d}_{6}\right): \quad \delta=5.35(\mathrm{~s}, 5 \mathrm{H}, 5 \mathrm{CH}) \mathrm{ppm}$.

${ }^{13} \mathrm{C}\left\{{ }^{1} \mathrm{H}\right\}$ NMR

(100.62 MHz, DMSO- $\left.\boldsymbol{d}_{6}\right)$ : $\quad \delta=103.0(\mathrm{~s}, 5 \mathrm{C}, 5 \mathrm{CH}) \mathrm{ppm}$.

${ }^{23} \mathrm{Na}$ NMR

(105.84 MHz, DMSO- $\left.\boldsymbol{d}_{6}\right)$ : $\quad \delta=-1$ to $-4\left(\mathrm{~s}_{\mathrm{br}}\right) \mathrm{ppm}$. 


\subsubsection{Synthesis and Characterization of $\mathrm{CpK}$}

Cyclopentadienylpotassium $(\mathrm{CpK})$ was prepared by addition of freshly distilled $\mathrm{CpH}(0.96 \mathrm{~g}$, $14.50 \mathrm{mmol}, 1.00$ eq.; from dicyclopentadiene see 4.1 .1$)$ to a solution of freshly cut potassium $(2.3 \mathrm{~g}$, $58.8 \mathrm{mmol}$, 4.1 eq.) in $\mathrm{Et}_{2} \mathrm{O}(15 \mathrm{~mL})$. After stirring the reaction mixture for $10 \mathrm{~h}$ and filtration of unreacted potassium, hexane $(10 \mathrm{~mL})$ was added. The product precipitated as a white solid $(0.33 \mathrm{~g}$, $3.17 \mathrm{mmol}, 22 \%)$ and was filtered and dried in vacuo.

Sum formula:

$\mathrm{C}_{5} \mathrm{H}_{5} \mathrm{~K}$

Molecular weight:

$104.19 \mathrm{~g} / \mathrm{mol}$

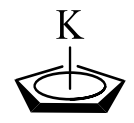

Dissolving the compound in different solvents (DMSO- $d_{6}$, THF- $d_{8}$ ) resulted in different solution state structures and therefore different chemical shifts:

(THF- $\left.d_{8}\right)$ :

Proposed aggregate:

Sum formula:

Molecular weight:

${ }^{1} \mathrm{H}$ NMR

(400.13 MHz, THF- $\left.d_{8}\right)$ :

${ }^{13} \mathrm{C}\left\{{ }^{1} \mathrm{H}\right\}$ NMR

$\left(100.62 \mathrm{MHz}\right.$, THF- $\left.d_{8}\right)$ :

(DMSO- $\left.d_{6}\right)$ :

${ }^{1} \mathrm{H}$ NMR

(400.13 MHz, DMSO- $\left.d_{6}\right)$ :

${ }^{13} \mathrm{C}\left\{{ }^{1} \mathrm{H}\right\}$ NMR

(100.62 MHz, DMSO- $\left.d_{6}\right)$ :

\section{$\left[\mathrm{C}_{5} \mathrm{H}_{5} \mathrm{~K}(\mathrm{THF})_{3}\right]$}

$\mathrm{C}_{17} \mathrm{H}_{29} \mathrm{O}_{3} \mathrm{~K}$

$320.51 \mathrm{~g} / \mathrm{mol}$

$\delta=5.63(\mathrm{~s}, 5 \mathrm{H}, 5 \mathrm{CH}) \mathrm{ppm}$.

Due to low solubility no resonance could be detected.

$\delta=5.39(\mathrm{~s}, 5 \mathrm{H}, 5 \mathrm{CH}) \mathrm{ppm}$.

$\delta=103.2(\mathrm{~s}, 5 \mathrm{C}, 5 \mathrm{CH}) \mathrm{ppm}$ 


\subsubsection{Synthesis and Characterization of $\mathrm{CpRb}$}

Cyclopentadienylrubidium ( $\mathrm{CpRb}$ ) was prepared by addition of freshly distilled $\mathrm{CpH}(0.06 \mathrm{~mL}$, $0.73 \mathrm{mmol}, 1.06$ eq.; from dicyclopentadiene see 4.1 .1$)$ to a stirred and cooled $\left(0^{\circ} \mathrm{C}\right)$ solution of RbHMDS (0.17 g, $0.69 \mathrm{mmol}, 1.00 \mathrm{eq}$.) in THF $(10 \mathrm{~mL})$. After heating to ambient temperature and stirring for another hour, pentane $(15 \mathrm{~mL})$ was added. The product precipitated as a light brown solid (0.09 g, $0.6 \mathrm{mmol}, 87 \%)$ and was filtered and dried in vacuo.

Sum formula:

$\mathrm{C}_{5} \mathrm{H}_{5} \mathrm{Rb}$

Molecular weight:

$150.56 \mathrm{~g} / \mathrm{mol}$

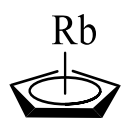

Dissolving the compound in different solvents (DMSO- $d_{6}$, THF- $d_{8}$ ) resulted in different solution state structures and therefore different chemical shifts:

(THF- $\left.d_{8}\right)$ :

Proposed aggregate:

$\left[\mathrm{C}_{5} \mathrm{H}_{5} \mathrm{Rb}(\mathrm{THF})_{2}\right]$

Sum formula:

$\mathrm{C}_{13} \mathrm{H}_{21} \mathrm{O}_{2} \mathrm{Rb}$

Molecular weight:

$294.77 \mathrm{~g} / \mathrm{mol}$

\section{${ }^{1} \mathrm{H}$ NMR}

(400.13 MHz, THF- $\left.\boldsymbol{d}_{8}\right)$ : $\quad \delta=5.61(\mathrm{~s}, 5 \mathrm{H}, 5 \mathrm{CH}) \mathrm{ppm}$.

${ }^{13} \mathrm{C}\left\{{ }^{1} \mathrm{H}\right\} \mathrm{NMR}$

(100.62 MHz, THF- $\left.\boldsymbol{d}_{\mathbf{8}}\right)$ : $\quad$ Due to low solubility no resonance could be detected.

(DMSO- $\left.d_{6}\right)$ :

${ }^{1} \mathrm{H}$ NMR

(400.13 MHz, DMSO- $\left.\boldsymbol{d}_{6}\right)$ : $\quad \delta=5.38(\mathrm{~s}, 5 \mathrm{H}, 5 \mathrm{CH}) \mathrm{ppm}$.

${ }^{13} \mathrm{C}\left\{{ }^{1} \mathrm{H}\right\} \mathrm{NMR}$

$\left(\mathbf{1 0 0 . 6 2} \mathrm{MHz}, \mathrm{DMSO}-\boldsymbol{d}_{6}\right): \quad \delta=103.3(\mathrm{~s}, 5 \mathrm{C}, 5 \mathrm{CH}) \mathrm{ppm}$. 


\subsubsection{Synthesis and Characterization of $\mathrm{CpCs}$}

Cyclopentadienylcaesium (CpCs) was prepared by addition of freshly distilled $\mathrm{CpH}(0.06 \mathrm{~mL}$, $0.73 \mathrm{mmol}, 1.06$ eq.; from dicyclopentadiene see 4.1 .1$)$ to a stirred and cooled $\left(0{ }^{\circ} \mathrm{C}\right)$ solution of CsHMDS (0.20 g, $0.69 \mathrm{mmol}, 1.00$ eq.) in THF $(10 \mathrm{~mL})$. After heating to ambient temperature and stirring for another hour, pentane $(15 \mathrm{~mL})$ was added. The product precipitated as a light brown solid (0.11 g, $0.56 \mathrm{mmol}, 81 \%)$ and was filtered and dried in vacuo.

Sum formula:

$\mathrm{C}_{5} \mathrm{H}_{5} \mathrm{Cs}$

Molecular weight:

$198.00 \mathrm{~g} / \mathrm{mol}$

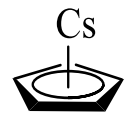

Dissolving the compound in different solvents (DMSO- $d_{6}$, THF- $d_{8}$ ) resulted in different solution state structures and therefore different chemical shifts:

(THF- $\left.d_{8}\right)$ :

${ }^{1} \mathrm{H}$ NMR

(400.13 MHz, THF- $\left.\boldsymbol{d}_{8}\right): \quad \delta=5.55(\mathrm{~s}, 5 \mathrm{H}, 5 \mathrm{CH}) \mathrm{ppm}$.

${ }^{13} \mathrm{C}\left\{{ }^{1} \mathrm{H}\right\}$ NMR

$\left(\mathbf{1 0 0 . 6 2} \mathrm{MHz}\right.$, THF- $\left.\boldsymbol{d}_{8}\right): \quad \delta=107.0(\mathrm{~s}, 5 \mathrm{C}, 5 \mathrm{CH}) \mathrm{ppm}$.

${ }^{133}$ Cs NMR

(52.48 MHz, THF- $\left.\boldsymbol{d}_{\mathbf{8}}\right): \quad \delta=-240.1(\mathrm{~s}) \mathrm{ppm}$.

(DMSO- $\left.d_{6}\right)$ :

${ }^{1} \mathrm{H}$ NMR

(400.13 MHz, DMSO- $\left.\boldsymbol{d}_{6}\right): \quad \delta=5.41(\mathrm{~s}, 5 \mathrm{H}, 5 \mathrm{CH}) \mathrm{ppm}$.

${ }^{13} \mathrm{C}\left\{{ }^{1} \mathrm{H}\right\} \mathrm{NMR}$

(100.62 MHz, DMSO- $\left.\boldsymbol{d}_{6}\right)$ : $\quad \delta=103.9(\mathrm{~s}, 5 \mathrm{C}, 5 \mathrm{CH}) \mathrm{ppm}$.

${ }^{133}$ Cs NMR

(52.48 MHz, DMSO- $\boldsymbol{d}_{6}$ ): $\quad \delta=-14.7$ (s) ppm. 


\subsubsection{Synthesis and Characterization of MeCpLi}

\subsubsection{Synthesis and Characterization of MeCpLi}

Methylcyclopentadienyllithium (MeCpLi) was prepared by lithiation of freshly distilled methylcyclopentadiene ( $1.68 \mathrm{~g}, 21.00 \mathrm{mmol}, 1.20 \mathrm{eq}$.; from methylcyclopentadiene dimer see 4.1.1) with $n \mathrm{BuLi}$ (4.74 M in hexane, $3.40 \mathrm{~mL}, 17.50 \mathrm{mmol}, 1.00 \mathrm{eq}$.) at $0{ }^{\circ} \mathrm{C}$ in THF $(50 \mathrm{~mL})$. After heating to ambient temperature, hexane $(10 \mathrm{~mL})$ was added to the solution. The product precipitated as a white solid (1.63 g, $18.94 \mathrm{mmol}, 90 \%)$ and was filtered and dried in vacuo. Carbon and hydrogen atoms are numbered according to IUPAC.

Sum formula:

Molecular weight:

Proposed aggregate:

Sum formula:

Molecular weight:

\section{${ }^{1} \mathrm{H}$ NMR}

(400.13 MHz, THF- $\left.d_{8}\right)$ :

$$
\mathrm{C}_{6} \mathrm{H}_{8} \mathrm{Li}
$$

$86.06 \mathrm{~g} / \mathrm{mol}$

$\left[\mathrm{C}_{6} \mathrm{H}_{8} \mathrm{Li}(\mathrm{THF})_{2}\right]$

$\mathrm{C}_{14} \mathrm{H}_{23} \mathrm{O}_{2} \mathrm{Li}$

$230.27 \mathrm{~g} / \mathrm{mol}$ $\delta=5.51$ to 5.48 (m, $2 \mathrm{H}, \mathrm{H}-3, \mathrm{H}-4), 5.47$ to 5.44 (m, $2 \mathrm{H}, \mathrm{H}-2, \mathrm{H}-5)$, $2.11\left(\mathrm{~s}, 3 \mathrm{H}, \mathrm{CH}_{3}\right) \mathrm{ppm}$.

${ }^{13} \mathrm{C}\left\{{ }^{1} \mathrm{H}\right\}$ NMR

(100.62 MHz, THF- $\left.d_{8}\right)$ : $\delta=113.8(\mathrm{C} 1), 103.8(\mathrm{C} 2, \mathrm{C} 5), 102.4(\mathrm{C} 3, \mathrm{C} 4), 15.6\left(\mathrm{CH}_{3}\right) \mathrm{ppm}$.

${ }^{7} \mathrm{Li}$ NMR

(155.51 MHz, THF- $\left.\boldsymbol{d}_{8}\right): \quad \delta=-7.60$ (s) $\mathrm{ppm}$. 


\subsubsection{Syntheses and Characterizations of $\mathrm{Cp} * \mathrm{M}(\mathrm{M}=\mathrm{Li}, \mathrm{K}, \mathrm{Cs})$}

\subsubsection{Synthesis of $C p^{*} \mathrm{Li}$}

Pentamethylcyclopentadienyllithium $\left(\mathrm{Cp}^{\star} \mathrm{Li}\right)$ was prepared following a preparation route similar to Robbins ${ }^{\text {[159] }}$. It was synthesized by lithiation of freshly distilled pentamethylcyclopentadiene (0.92 g, $6.80 \mathrm{mmol}, 1.13$ eq.) with $n \mathrm{BuLi}\left(5.1 \mathrm{M}\right.$ in hexane, $1.2 \mathrm{~mL}, 6.0 \mathrm{mmol}, 1.0 \mathrm{eq}$.) at $0{ }^{\circ} \mathrm{C}$ in THF $(15 \mathrm{~mL})$. After heating to ambient temperature, pentane $(10 \mathrm{~mL})$ was added to the solution. The product precipitated as a white solid $(0.53 \mathrm{~g}, 3.73 \mathrm{mmol}, 62 \%)$ and was filtered and dried in vacuo.

Sum formula:

$\mathrm{C}_{10} \mathrm{H}_{15} \mathrm{Li}$

Molecular weight:

$142.17 \mathrm{~g} / \mathrm{mol}$

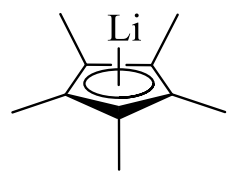

$\mathrm{Cp}^{\star} \mathrm{Li}$ was not soluble in THF- $d_{8}$. Therefore, no further structure elucidation was pursued.

\subsubsection{Synthesis and Characterization of $\mathrm{Cp}^{\star} \mathrm{K}$}

Pentamethylcyclopentadienylpotassium $\left(\mathrm{Cp}^{\star} \mathrm{K}\right)$ was prepared by addition of freshly distilled pentamethylcyclopentadiene $(1.31 \mathrm{~g}, 9.60 \mathrm{mmol}, 1.20 \mathrm{eq}$.) to a solution of freshly cut potassium (0.32 g, $8.20 \mathrm{mmol}, 1.0$ eq.) in $\mathrm{Et}_{2} \mathrm{O}(15 \mathrm{~mL})$. After stirring the reaction mixture for $15 \mathrm{~h}$, pentane $(15 \mathrm{~mL})$ was added. The product precipitated as a white solid $(1.06 \mathrm{~g}, 6.10 \mathrm{mmol}, 74 \%)$ and was filtered and dried in vacuo.

Sum formula:

Molecular weight:

Proposed aggregate:

Sum formula:

Molecular weight:

${ }^{1} \mathrm{H}$ NMR

(400.13 MHz, THF- $\left.d_{8}\right)$ :

${ }^{13} \mathrm{C}\left\{{ }^{1} \mathrm{H}\right\}$ NMR

$\left(100.62 \mathrm{MHz}\right.$, THF- $\left.d_{8}\right)$ :
$\mathrm{C}_{10} \mathrm{H}_{15} \mathrm{~K}$

$174.32 \mathrm{~g} / \mathrm{mol}$

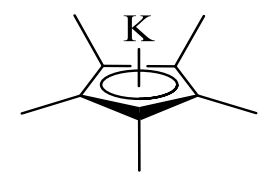

$\left[\mathrm{C}_{10} \mathrm{H}_{15} \mathrm{~K}(\mathrm{THF})_{3}\right]$

$\mathrm{C}_{22} \mathrm{H}_{39} \mathrm{O}_{3} \mathrm{~K}$

$390.64 \mathrm{~g} / \mathrm{mol}$

$\delta=104.8\left(5 \mathrm{C}_{\text {tert }}\right), 11.4\left(5 \mathrm{CH}_{3}\right) \mathrm{ppm}$. 


\subsubsection{Characterization of $\mathrm{Cp}{ }^{\star} \mathrm{Cs}$}

Pentamethylcyclopentadienylcaesium $\left(\mathrm{Cp}^{\star} \mathrm{Cs}\right)$ was synthesized by Neufeld from our group and only further characterized in this work.

Sum formula:

Molecular weight:

Proposed aggregate:

Sum formula:

Molecular weight:

\section{${ }^{1} \mathrm{H}$ NMR}

(400.13 MHz, THF- $\left.d_{8}\right)$ :

${ }^{13} \mathrm{C}\left\{{ }^{1} \mathrm{H}\right\} \mathrm{NMR}$

(100.62 MHz, THF- $\left.d_{8}\right)$ :
$\mathrm{C}_{10} \mathrm{H}_{15} \mathrm{Cs}$

$268.13 \mathrm{~g} / \mathrm{mol}$

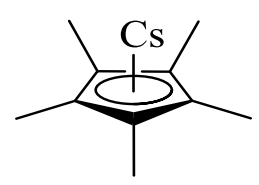

$\left[\mathrm{C}_{10} \mathrm{H}_{15} \mathrm{Cs}(\mathrm{THF})_{4}\right]$

$\mathrm{C}_{26} \mathrm{H}_{47} \mathrm{O}_{4} \mathrm{Cs}$

$556.55 \mathrm{~g} / \mathrm{mol}$

$\delta=1.85\left(\mathrm{~s}, 15 \mathrm{H}, 5 \mathrm{CH}_{3}\right) \mathrm{ppm}$.

$\delta=108.0 \quad\left(5 \mathrm{C}_{\text {tert }}\right), \quad 11.1 \quad\left(5 \mathrm{CH}_{3}\right) \mathrm{ppm}$. ( $\mathrm{C}_{\text {tert }}$ were observed via ${ }^{1} \mathrm{H},{ }^{13} \mathrm{C}-\mathrm{HMBC}$ experiments) 


\subsubsection{Characterizations of $\mathrm{RMgCl}(\mathrm{R}=\mathrm{Et}, i \mathrm{Pr}, n \mathrm{Bu}, n \mathrm{Hex}, n O c t, n \mathrm{Dec})$}

Ethylmagnesium chloride (EtMgCl; in THF, 2 M) was purchased from Sigma-Aldrich and used as received for characterization. $\boldsymbol{i}$-Propyl- $(i \mathrm{PrMgCl}), \boldsymbol{n}$-butyl- $(n \mathrm{BuMgCl}), \boldsymbol{n}$-hexyl- $(n \mathrm{HexMgCl}), \boldsymbol{n}$ octyl- $(n \mathrm{OctMgCl})$ and $\boldsymbol{n}$-decylmagnesium chloride $(n \mathrm{DecMgCl})$ were synthesized by Schnegelsberg from the Koszinowski group and only further characterized in this work. ${ }^{[3]}$ Carbon and hydrogen atoms of the alkyl chains are numbered according to IUPAC.

\subsubsection{Characterization of EtMgCl}

Proposed aggregate:

Sum formula:

Molecular weight:

${ }^{1} \mathrm{H}$ NMR

(400.13 MHz, THF- $\left.d_{8}\right)$ :

${ }^{13} \mathrm{C}\left\{{ }^{1} \mathrm{H}\right\}$ NMR

$\left(100.62 \mathrm{MHz}\right.$, THF- $\left.d_{8}\right)$ :
[ $\mathrm{C}_{2} \mathrm{H}_{5} \mathrm{MgCl}(\mathrm{THF})_{2}$ ]

$\mathrm{C}_{10} \mathrm{H}_{21} \mathrm{O}_{2} \mathrm{MgCl}$

$233.03 \mathrm{~g} / \mathrm{mol}$

\subsubsection{Characterization of $i \mathrm{PrMgCl}$}

Proposed aggregate:

Sum formula:

Molecular weight:

${ }^{1} \mathrm{H}$ NMR

(400.13 MHz, THF- $\left.d_{8}\right)$ :

${ }^{13} \mathrm{C}\left\{{ }^{1} \mathrm{H}\right\}$ NMR

(100.62 MHz, THF- $\left.d_{8}\right)$ :
$\left[\mathrm{C}_{3} \mathrm{H}_{7} \mathrm{MgCl}(\mathrm{THF})_{2}\right]$

$\mathrm{C}_{11} \mathrm{H}_{23} \mathrm{O}_{2} \mathrm{MgCl}$

$247.06 \mathrm{~g} / \mathrm{mol}$

$\delta=1.26\left(\mathrm{~d},{ }^{3} \mathrm{~J}_{\mathrm{HH}}=7.7 \mathrm{~Hz}, 6 \mathrm{H}, \mathrm{H} .2, \mathrm{H}-2{ }^{\prime}\right),-0.31$ to $-0.47(\mathrm{~m}, 1 \mathrm{H}$, $\mathrm{H}-1) \mathrm{ppm}$.

$\delta=26.2\left(\mathrm{C} 2, \mathrm{C} 2{ }^{\prime}\right), 9.9(\mathrm{C} 1) \mathrm{ppm}$. 


\subsubsection{Characterization of $n \mathrm{BuMgCl}$}

Proposed aggregate: $\quad\left[\mathrm{C}_{4} \mathrm{H}_{9} \mathrm{MgCl}(\mathrm{THF})_{2}\right]$

Sum formula: $\quad \mathrm{C}_{12} \mathrm{H}_{25} \mathrm{O}_{2} \mathrm{MgCl}$

Molecular weight: $\quad 261.08 \mathrm{~g} / \mathrm{mol}$

${ }^{1} \mathrm{H}$ NMR

(400.13 MHz, THF- $\left.d_{8}\right)$ : $\quad \delta=1.58$ to 1.47 (m, $\left.2 \mathrm{H}, \mathrm{H}-2\right), 1.31$ to $1.19(\mathrm{~m}, 2 \mathrm{H}, \mathrm{H}-3), 0.85$ (t, $\left.{ }^{3} J_{\mathrm{HH}}=7.3 \mathrm{~Hz}, 3 \mathrm{H}, \mathrm{H}-4\right),-0.57$ to -0.65 (m, $\left.2 \mathrm{H}, \mathrm{H}-1\right) \mathrm{ppm}$.

${ }^{13} \mathrm{C}\left\{{ }^{1} \mathrm{H}\right\}$ NMR

$\left(\mathbf{1 0 0 . 6 2} \mathrm{MHz}\right.$, THF- $\left.\boldsymbol{d}_{\mathbf{8}}\right): \quad \delta=33.9(\mathrm{C} 2), 32.5(\mathrm{C} 3), 14.7(\mathrm{C} 4), 7.9(\mathrm{C} 1) \mathrm{ppm}$.

\subsubsection{Characterization of $n \mathrm{HexMgCl}$}

Proposed aggregate: $\quad\left[\mathrm{C}_{6} \mathrm{H}_{13} \mathrm{MgCl}(\mathrm{THF})_{2}\right]$

Sum formula: $\quad \mathrm{C}_{14} \mathrm{H}_{29} \mathrm{O}_{2} \mathrm{MgCl}$

Molecular weight: $\quad 289.14 \mathrm{~g} / \mathrm{mol}$

${ }^{1} \mathrm{H}$ NMR

(400.13 MHz, THF- $\left.d_{8}\right): \quad \delta=1.60$ to $1.49(\mathrm{~m}, 2 \mathrm{H}, \mathrm{H}-2), 1.33$ to $1.23(\mathrm{~m}, 6 \mathrm{H}, \mathrm{H}-3, \mathrm{H}-4, \mathrm{H}-5)$, 0.91 to 0.85 (m, $3 \mathrm{H}, \mathrm{H}-6),-0.62\left(\mathrm{t},{ }^{3} \mathrm{~J}_{\mathrm{HH}}=8.5 \mathrm{~Hz}, 2 \mathrm{H}, \mathrm{H}-1\right) \mathrm{ppm}$.

${ }^{13} \mathbf{C}\left\{{ }^{1} \mathbf{H}\right\}$ NMR

$\left(\mathbf{1 0 0 . 6 2} \mathrm{MHz}\right.$, THF- $\left.\boldsymbol{d}_{\mathbf{8}}\right): \quad \delta=38.6$ (C3), 32.2 (C4), 30.2 (C2), 23.0 (C5), 13.8 (C6), 7.2 (C1) ppm.

\subsubsection{Characterization of $n O c t M g C l$}

Proposed aggregate: $\quad\left[\mathrm{C}_{8} \mathrm{H}_{17} \mathrm{MgCl}(\mathrm{THF})_{2}\right]$

Sum formula: $\quad \mathrm{C}_{16} \mathrm{H}_{33} \mathrm{O}_{2} \mathrm{MgCl}$

Molecular weight: $\quad 317.19 \mathrm{~g} / \mathrm{mol}$

${ }^{1}$ H NMR

(400.13 MHz, THF- $\left.\boldsymbol{d}_{\mathbf{8}}\right): \quad \delta=1.60$ to $1.49(\mathrm{~m}, 2 \mathrm{H}, \mathrm{H}-2), 1.36$ to $1.16(\mathrm{~m}, 10 \mathrm{H}, \mathrm{H}-3$ to H-7), 0.90 to $0.87(\mathrm{~m}, 3 \mathrm{H}, \mathrm{H}-8),-0.62\left(\mathrm{t},{ }^{3} \mathrm{~J}_{\mathrm{HH}}=8.6 \mathrm{~Hz}, 2 \mathrm{H}, \mathrm{H}-1\right) \mathrm{ppm}$.

${ }^{13} \mathrm{C}\left\{{ }^{1} \mathrm{H}\right\}$ NMR

$\left(\mathbf{1 0 0 . 6 2} \mathrm{MHz}\right.$, THF- $\left.\boldsymbol{d}_{\mathbf{8}}\right): \quad \delta=40.0(\mathrm{C} 3), 33.3(\mathrm{C} 7), 31.3$ (C5), 30.9 (C2), $30.8(\mathrm{C} 4), 23.7$ (C6), $14.8(\mathrm{C} 8), 8.2(\mathrm{C} 1) \mathrm{ppm}$. 


\subsubsection{Characterization of $n \mathrm{DecMgCl}$}

\begin{tabular}{|c|c|}
\hline Proposed aggregate: & {$\left[\mathrm{C}_{10} \mathrm{H}_{21} \mathrm{MgCl}(\mathrm{THF})_{2}\right]$} \\
\hline Sum formula: & $\mathrm{C}_{18} \mathrm{H}_{37} \mathrm{O}_{2} \mathrm{MgCl}$ \\
\hline Molecular weight: & $317.19 \mathrm{~g} / \mathrm{mol}$ \\
\hline \multicolumn{2}{|l|}{${ }^{1} \mathbf{H}$ NMR } \\
\hline \multirow[t]{2}{*}{$\left(400.13 \mathrm{MHz}\right.$, THF- $\left.d_{8}\right):$} & $\delta=1.56$ to $1.39(\mathrm{~m}, 2 \mathrm{H}, \mathrm{H}-2), 1.36$ to $1.23(\mathrm{~m}, 14 \mathrm{H}, \mathrm{H}-3$ to $\mathrm{H}-9)$, \\
\hline & 0.93 to 0.85 (m, $3 \mathrm{H}, \mathrm{H}-10),-0.59$ to $-0.67(\mathrm{~m}, 2 \mathrm{H}, \mathrm{H}-1) \mathrm{ppm}$. \\
\hline \multicolumn{2}{|l|}{${ }^{13} \mathrm{C}\left\{{ }^{1} \mathrm{H}\right\}$ NMR } \\
\hline$\left(100.62 \mathrm{MHz}\right.$, THF- $\left.d_{8}\right):$ & $\delta=40.0(\mathrm{C} 3), 33.0(\mathrm{C} 9), 31.3(\mathrm{C} 2), 31.2(\mathrm{C} 5$ or $\mathrm{C} 6$ or $\mathrm{C} 7), 30.9(\mathrm{C} 5$ \\
\hline & $\begin{array}{l}\text { or C6 or C7), } 30.9(\mathrm{C} 4), 30.5 \text { (C5 or C6 or C7), } 23.7(\mathrm{C} 8), 14.5 \\
(\mathrm{C} 10), 8.2(\mathrm{C} 1) \mathrm{ppm} \text {. }\end{array}$ \\
\hline
\end{tabular}

\subsubsection{Characterization of $n \mathrm{BuMgBr}$}

$\boldsymbol{n}$ Butylmagnesium bromide $(n \mathrm{BuMgBr})$ was synthesized by Schnegelsberg from the Koszinowski group and only further characterized in this work ${ }^{[3]}$ Carbon and hydrogen atoms of the alkyl chain are numbered according to IUPAC.

\subsubsection{Characterization of $n \mathrm{BuMgBr}$}

Proposed aggregate:

Sum formula:

Molecular weight:

${ }^{1}$ H NMR

$\left(400.13 \mathrm{MHz}\right.$, THF- $\left.d_{8}\right)$ :

${ }^{13} \mathrm{C}\left\{{ }^{1} \mathrm{H}\right\}$ NMR

(100.62 MHz, THF- $\left.d_{8}\right)$ :
[ $\mathrm{C}_{4} \mathrm{H}_{9} \mathrm{MgBr}(\mathrm{THF})_{2}$ ]

$\mathrm{C}_{12} \mathrm{H}_{25} \mathrm{O}_{2} \mathrm{MgBr}$

$305.53 \mathrm{~g} / \mathrm{mol}$

$\delta=1.57$ to $1.48(\mathrm{~m}, 2 \mathrm{H}, \mathrm{H}-2), 1.26$ (sextet, ${ }^{3} J_{\mathrm{HH}}=7.4 \mathrm{~Hz}, 2 \mathrm{H}, \mathrm{H}-3$ ), $0.85\left(\mathrm{t},{ }^{3} J_{\mathrm{HH}}=7.3 \mathrm{~Hz}, 3 \mathrm{H}, \mathrm{H}-4\right),-0.59\left(\mathrm{t},{ }^{3} J_{\mathrm{HH}}=8.2 \mathrm{~Hz}, 2 \mathrm{H}\right.$, $\mathrm{H}-1)$ ppm.

$\delta=33.8(\mathrm{C} 2), 32.4(\mathrm{C} 3), 14.7(\mathrm{C} 4), 8.1(\mathrm{C} 1) \mathrm{ppm}$. 


\subsubsection{Characterizations of $[n \mathrm{BuMgX} \cdot \mathrm{LiX}](\mathrm{X}=\mathrm{Cl}, \mathrm{Br})$}

$[n \mathrm{BuMgX} \cdot \mathrm{LiX}](\mathrm{X}=\mathrm{Cl}, \mathrm{Br})$ were prepared by addition of equimolar amounts of dry LiX in THF to solutions of $n \mathrm{BuMgX}$. Carbon and hydrogen atoms of the alkyl chains are numbered according to IUPAC.

\subsubsection{Characterization of $[\mathrm{nBuMgCl} \cdot \mathrm{LiCl}]$}

Proposed aggregate: $\quad\left[\mathrm{C}_{4} \mathrm{H}_{9} \mathrm{MgCl}(\mathrm{THF})_{2} \cdot \mathrm{LiCl}\right]$

Sum formula: $\quad \mathrm{C}_{12} \mathrm{H}_{25} \mathrm{O}_{2} \mathrm{MgLiCl}_{2}$

Molecular weight: $\quad 303.48 \mathrm{~g} / \mathrm{mol}$

${ }^{1} \mathrm{H}$ NMR

(400.13 MHz, THF- $d_{8}$ ): $\quad \delta=1.52 \quad$ (quintet, ${ }^{3} J_{\mathrm{HH}}=7.4 \mathrm{~Hz}, \quad 2 \mathrm{H}, \quad \mathrm{H}-2$ ), 1.25 (sextet, $\left.{ }^{3} J_{\mathrm{HH}}=7.4 \mathrm{~Hz}, 2 \mathrm{H}, \mathrm{H}-3\right), 0.84\left(\mathrm{t},{ }^{3} J_{\mathrm{HH}}=7.4 \mathrm{~Hz}, 3 \mathrm{H}, \mathrm{H}-4\right),-0.59(\mathrm{t}$, $\left.{ }^{3} J_{\mathrm{HH}}=8.3 \mathrm{~Hz}, 2 \mathrm{H}, \mathrm{H}-1\right)$ ppm.

${ }^{13} \mathrm{C}\left\{{ }^{1} \mathrm{H}\right\} \mathrm{NMR}$

$\left(\mathbf{1 0 0 . 6 2} \mathbf{~ M H z}\right.$, THF- $\left.\boldsymbol{d}_{\mathbf{8}}\right): \quad \delta=33.8(\mathrm{C} 2), 32.3(\mathrm{C} 3), 14.7(\mathrm{C} 4), 8.1(\mathrm{C} 1) \mathrm{ppm}$.

${ }^{7} \mathrm{Li}$ NMR

$\left(\mathbf{1 5 5 . 5 1} \mathrm{MHz}, \mathrm{THF}-\boldsymbol{d}_{8}\right): \quad \delta=0.28(\mathrm{~s}) \mathrm{ppm}$.

\subsubsection{Characterization of $[n \mathrm{BuMgBr} \cdot \mathrm{LiBr}]$}

$\begin{array}{ll}\text { Proposed aggregate: } & {\left[\mathrm{C}_{4} \mathrm{H}_{9} \mathrm{MgBr}(\mathrm{THF})_{2} \cdot \mathrm{LiBr}\right]} \\ \text { Sum formula: } & \mathrm{C}_{12} \mathrm{H}_{25} \mathrm{O}_{2} \mathrm{MgLiBr}_{2} \\ \text { Molecular weight: } & 392.38 \mathrm{~g} / \mathrm{mol}\end{array}$

${ }^{1} \mathrm{H}$ NMR

(400.13 MHz, THF- $\boldsymbol{d}_{\mathbf{8}}$ ): $\delta=1.52$ (quintet, ${ }^{3} J_{\mathrm{HH}}=8.0 \mathrm{~Hz}, 2 \mathrm{H}, \quad \mathrm{H}-2$ ), 1.25 (sextet, $\left.{ }^{3} J_{\mathrm{HH}}=7.1 \mathrm{~Hz}, 2 \mathrm{H}, \mathrm{H}-3\right), 0.85\left(\mathrm{t},{ }^{3} J_{\mathrm{HH}}=7.1 \mathrm{~Hz}, 3 \mathrm{H}, \mathrm{H}-4\right),-0.59(\mathrm{t}$, $\left.{ }^{3} J_{\mathrm{HH}}=8.0 \mathrm{~Hz}, 2 \mathrm{H}, \mathrm{H}-1\right) \mathrm{ppm}$.

${ }^{13} \mathrm{C}\left\{{ }^{1} \mathrm{H}\right\}$ NMR

$\left(\mathbf{1 0 0 . 6 2} \mathrm{MHz}\right.$, THF- $\left.\boldsymbol{d}_{\mathbf{8}}\right): \quad \delta=33.7(\mathrm{C} 2), 32.3(\mathrm{C} 3), 14.6(\mathrm{C} 4), 8.3(\mathrm{C} 1) \mathrm{ppm}$.

${ }^{7} \mathrm{Li}$ NMR

$\left(\mathbf{1 5 5 . 5 1} \mathrm{MHz}\right.$, THF- $\left.\boldsymbol{d}_{\mathbf{8}}\right): \quad \delta=0.61(\mathrm{~s}) \mathrm{ppm}$. 


\subsubsection{Characterizations of $\left[\mathrm{MX}_{2}\left\{\left(\mathrm{Me}_{2} \mathrm{NCH}_{2}\right)_{2} \mathrm{Acr}\right\}\right]\left(\mathrm{MX}{ }_{2}=\mathrm{ZnBr}(\mathrm{DMF}), \mathrm{CdBr}_{2}\right)$}

$\left[\mathrm{ZnBr}(\mathrm{DMF})\left\{\left(\mathrm{Me}_{2} \mathrm{NCH}_{2}\right)_{2} \mathrm{Acr}\right\}\right]$ and $\left[\mathrm{CdBr}_{2}\left\{\left(\mathrm{Me}_{2} \mathrm{NCH}_{2}\right)_{2} \mathrm{Acr}\right\}\right]$ were synthesized by Visscher from our group and only further characterized in this work. ${ }^{[1,150]}$

\subsubsection{Characterization of $\left[\mathrm{ZnBr}(\mathrm{DMF})\left\{\left(\mathrm{Me}_{2} \mathrm{NCH}_{2}\right)_{2} \mathrm{Acr}\right\}\right]$}

Sum formula:

Molecular weight:

$\mathrm{C}_{22} \mathrm{H}_{30} \mathrm{~N}_{4} \mathrm{OZnBr}$

$511.78 \mathrm{~g} / \mathrm{mol}$

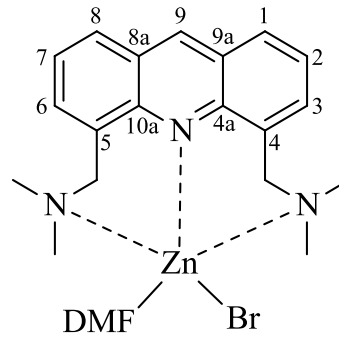

$\left(\mathrm{DMF}-\boldsymbol{d}_{7}, 80^{\circ} \mathrm{C}\right)$

${ }^{1} \mathrm{H}$ NMR

(400.13 MHz, DMF- $\left.d_{7}\right)$ :

$\delta=9.25(\mathrm{~s}, 1 \mathrm{H}, \mathrm{H}-9), 8.22\left(\mathrm{~d},{ }^{3} \mathrm{~J}_{\mathrm{HH}}=8.3 \mathrm{~Hz}, 2 \mathrm{H}, \mathrm{H}-1, \mathrm{H}-8\right), 8.01(\mathrm{~d}$, $\left.{ }^{3} J_{\mathrm{HH}}=7.1 \mathrm{~Hz}, 2 \mathrm{H}, \mathrm{H}-3, \mathrm{H}-6\right), 7.69\left(\mathrm{dd},{ }^{3} J_{\mathrm{HH}}=8.3 \mathrm{~Hz}, 7.1 \mathrm{~Hz}, 2 \mathrm{H}\right.$, $\mathrm{H}-2, \mathrm{H}-7), 4.52$ (s, $\left.4 \mathrm{H}, 2 \mathrm{CH}_{2}\right), 2.26\left(\mathrm{~s}, 12 \mathrm{H}, 4 \mathrm{CH}_{3}\right) \mathrm{ppm}$.

$\left(\mathrm{DMF}-\boldsymbol{d}_{7},-30^{\circ} \mathrm{C}\right)$ :

${ }^{1} \mathrm{H}$ NMR

(400.13 MHz, DMF- $\left.d_{7}\right)$

$\delta=9.57(\mathrm{~s}, 1 \mathrm{H}, \mathrm{H}-9), 8.39\left(\mathrm{dd},{ }^{3} J_{\mathrm{HH}}=8.0 \mathrm{~Hz},{ }^{4} J_{\mathrm{HH}}=1.6 \mathrm{~Hz}, 2 \mathrm{H}\right.$, $\mathrm{H}-1, \mathrm{H}-8), 8.11\left(\mathrm{dd},{ }^{3} J_{\mathrm{HH}}=6.1 \mathrm{~Hz},{ }^{4} J_{\mathrm{HH}}=1.3 \mathrm{~Hz}, 2 \mathrm{H}, \mathrm{H}-3, \mathrm{H}-6\right)$, $7.76\left(\mathrm{dd},{ }^{3} J_{\mathrm{HH}}=8.4 \mathrm{~Hz}, 6.8 \mathrm{~Hz}, 2 \mathrm{H}, \mathrm{H}-2, \mathrm{H}-7\right), 5.46\left(\mathrm{~d},{ }^{2} J_{\mathrm{HH}}=\right.$ $\left.11.9 \mathrm{~Hz}, 2 \mathrm{H}, \mathrm{CH}_{2}\right), 3.86\left(\mathrm{~d},{ }^{2} J_{\mathrm{HH}}=11.9 \mathrm{~Hz}, 2 \mathrm{H}, \mathrm{CH}_{2}\right), 3.19(\mathrm{~s}, 6 \mathrm{H}$, $\left.2 \mathrm{CH}_{3}\right), 2.38\left(\mathrm{~s}, 6 \mathrm{H}, 2 \mathrm{CH}_{3}\right) \mathrm{ppm}$.

${ }^{13} \mathrm{C}\left\{{ }^{1} \mathrm{H}\right\}$ NMR

(100.62 MHz, DMF- $\left.\boldsymbol{d}_{7}\right): \quad \delta=148.7$ (C4a, C10a) 142.0 (C9), 135.5 (C3, C6), 130.5 (C1, C8), 127.7 (C8a, C9a), 126.4 (C2, C7), $63.1\left(2 \mathrm{CH}_{2}\right), 48.9\left(2 \mathrm{CH}_{3}\right), 46.4$ $\left(2 \mathrm{CH}_{3}\right)$ ppm.

${ }^{15} \mathrm{~N}$ NMR

(40.56 MHz, DMF- $\boldsymbol{d}_{7}$ ): $\delta=-347 \mathrm{ppm}$. (Signal was observed via ${ }^{1} \mathrm{H},{ }^{15} \mathrm{~N}-\mathrm{HMBC}$ experiments) 


\subsubsection{Characterization of $\left[\mathrm{CdBr}_{2}\left\{\left(\mathrm{Me}_{2} \mathrm{NCH}_{2}\right)_{2} \mathrm{Acr}\right\}\right]$}

Sum formula:

Molecular weight:

$565.63 \mathrm{~g} / \mathrm{mol}$
$\mathrm{C}_{19} \mathrm{H}_{23} \mathrm{~N}_{3} \mathrm{CdBr}_{2}$

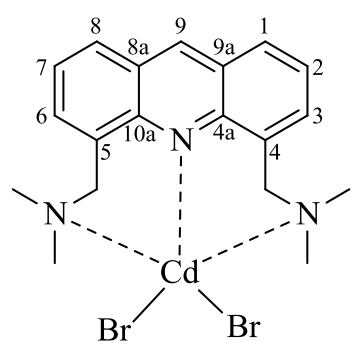

(DMF- $\left.d_{7}, 80^{\circ} \mathrm{C}\right)$ :

\section{${ }^{1}$ H NMR}

(400.13 MHz, DMF- $\left.\boldsymbol{d}_{7}\right): \quad \delta=9.32(\mathrm{~s}, 1 \mathrm{H}, \mathrm{H}-9), 8.28\left(\mathrm{~d},{ }^{3} J_{\mathrm{HH}}=8.5 \mathrm{~Hz}, 2 \mathrm{H}, \mathrm{H}-1, \mathrm{H}-8\right), 8.08$ to $7.99(\mathrm{~m}, 2 \mathrm{H}, \mathrm{H}-3, \mathrm{H}-6), 7.71\left(\mathrm{dd},{ }^{3} J_{\mathrm{HH}}=8.5 \mathrm{~Hz}, 6.8 \mathrm{~Hz}, 2 \mathrm{H}, \mathrm{H}-2\right.$, $\mathrm{H}-7), 4.56$ (s, $4 \mathrm{H}, 2 \mathrm{CH}_{2}$ ), $2.74\left(\mathrm{~s}, 12 \mathrm{H}, 4 \mathrm{CH}_{3}\right) \mathrm{ppm}$.

(DMF- $\left.d_{7},-30^{\circ} \mathrm{C}\right)$ :

${ }^{113}$ Cd NMR

(88.80 MHz, DMF- $\left.d_{7}\right)$ : $\delta=-345 \mathrm{ppm} . \quad$ (Signal was observed via $\quad{ }^{1} \mathrm{H},{ }^{113} \mathrm{Cd}-\mathrm{HMBC}$ experiments) 


\section{APPENDIX}

\section{Table of Contents}

$\begin{array}{ll}\text { Categorization of Model Compounds } & 101\end{array}$

Principal Moments of Inertia of Model Compounds $\quad 104$

Absolute Logarithmic Diffusion Coefficients of Model Compounds for Various Solvents $\quad 105$

ECCs and Normalized Diffusion Coefficients of Model Compounds for Various Solvents $\quad 106$

$\begin{array}{ll}\text { ECCs and Model Compounds for DMSO- } d_{6} & 107\end{array}$

ECCs and Model Compounds for DMSO- $d_{6}$ at Different Temperatures (Compact Spherical Molecules) 109

ECCs and Model Compounds for Cyclohexane- $d_{12} \quad 110$

ECCs and Model Compounds for $\mathrm{CDCl}_{3} \quad 112$

ECCs and Model Compounds for $\mathrm{CD}_{2} \mathrm{Cl}_{2} \quad 114$

ECCs and Model Compounds for $\mathrm{C}_{6} \mathrm{D}_{6} \quad 116$

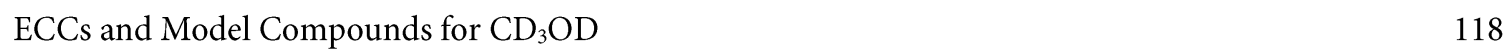

$\begin{array}{ll}\text { ECCs and Model Compounds for } \mathrm{CD}_{3} \mathrm{CN} & 120\end{array}$

ECCs and Model Compounds for THF- $d_{8}$ (Rod-Like and Brominated Rod-Like Molecules) 122

Theoretical Error Analysis of Model Compounds for Various Solvents 123

Molar Van-Der-Waals Densities $\left(M D_{w}\right)$ of Model Compounds 125

ECC-MW Estimations of CpM Derivatives (M = Li, Na, K, Rb, Cs) 127

ECC-MW Estimation of CpLi in THF and DMSO 127

$\begin{array}{lr}\text { ECC- } M W \text { Estimation of CpNa in THF and DMSO } & 129\end{array}$

ECC-MW Estimation of CpK in THF and DMSO 130

ECC-MW Estimation of CpRb in THF and DMSO 131

ECC-MW Estimation of CpCs in THF and DMSO 131

ECC-MW Estimation of MeCpLi in THF 132

ECC-MW Estimation of $\mathrm{Cp}^{*} \mathrm{~K}$ in THF 133

ECC- $M W$ Estimation of $\mathrm{Cp}^{*} \mathrm{Cs}$ in THF 133

Molar Van-Der-Waals Densities $\left(M D_{\mathrm{W}}\right) \quad 133$

ECC-MW Estimations of RMgX ( $\mathrm{R}=\mathrm{Et}, i \mathrm{Pr}, n \mathrm{Bu}, n \mathrm{Hex}, n \mathrm{Oct}, n \mathrm{Dec} ; \mathrm{X}=\mathrm{Cl}, \mathrm{Br})$

ECC-MW Estimation of EtMgCl in THF 134

$\begin{array}{ll}\text { ECC- } M W \text { Estimation of } i \mathrm{PrMgCl} \text { in THF } & 134\end{array}$

ECC- $M W$ Estimation of $n \mathrm{BuMgCl}$ in THF 134

$\begin{array}{ll}\text { ECC- } M W \text { Estimation of } n \mathrm{HexMgCl} \text { in THF } & 135\end{array}$

ECC-MW Estimation of $n \mathrm{OctMgCl}$ in THF $\quad 135$

ECC-MW Estimation of $n \mathrm{DecMgCl}$ in THF $\quad 135$

$\begin{array}{ll}\text { ECC- } M W \text { Estimation of } n \mathrm{BuMgBr} \text { in THF } & 136\end{array}$

Molar Van-Der-Waals Densities $\left(M D_{\mathrm{W}}\right) \quad 136$

ECC-MW Estimations of $[n \mathrm{BuMgX} \cdot \mathrm{LiX}](\mathrm{X}=\mathrm{Cl}, \mathrm{Br})$

ECC-MW Estimation of $[n \mathrm{BuMgCl} \cdot \mathrm{LiCl}]$ in THF 137

ECC-MW Estimation of $[n \mathrm{BuMgBr} \cdot \mathrm{LiBr}]$ in THF 137 
Molar Van-Der-Waals Densities $\left(M D_{\mathrm{W}}\right)$

ECC-MW Estimations of (IPrPh)X (X = Br, I, OTf, BF $\left.4, \mathrm{PF}_{6}\right) \quad 138$

ECC-MW Estimation of (IPrPh)Br in Dichloromethane 138

ECC-MW Estimation of (IPrPh)I in Dichloromethane 138

ECC-MW Estimation of (IPrPh)OTf in Dichloromethane 138

ECC-MW Estimation of (IPrPh)BF $\mathrm{BF}_{4}$ in Dichloromethane 139

ECC-MW Estimation of (IPrPh) $\mathrm{PF}_{6}$ in Dichloromethane 139

Molar Van-Der-Waals Densities $\left(M D_{\mathrm{W}}\right) \quad 139$

ECC-MW Estimations of (4,6-tBu-NCOC $\left.{ }_{6} \mathrm{H}_{2}\right)_{2} \mathrm{CH}_{2}$ and $\left[\mathrm{M}\left(4,6-\mathrm{tBu}-\mathrm{NCOC}_{6} \mathrm{H}_{2}\right)_{2} \mathrm{CH}\right](\mathrm{M}=\mathrm{Li}, \mathrm{K}, \mathrm{Mg}) 140$

ECC- $M W$ Estimation of the $\left(4,6-t \mathrm{Bu}-\mathrm{NCOC}_{6} \mathrm{H}_{2}\right)_{2} \mathrm{CH}_{2}$ Ligand in Benzene 140

ECC- $M W$ Estimation of $\left[\mathrm{Li}\left(4,6-t \mathrm{Bu}-\mathrm{NCOC}_{6} \mathrm{H}_{2}\right)_{2} \mathrm{CH}\right]$ in Benzene 140

ECC- $M W$ Estimation of $\left[\mathrm{K}\left(4,6-t \mathrm{Bu}-\mathrm{NCOC}_{6} \mathrm{H}_{2}\right)_{2} \mathrm{CH}\right]$ in Benzene 140

ECC- $M W$ Estimation of $\left[\mathrm{MgCl}_{2}\left(4,6-t \mathrm{Bu}-\mathrm{NCOC}_{6} \mathrm{H}_{2}\right)_{2} \mathrm{CH}\right]$ in Benzene 141

ECC- $M W$ Estimation of $\left[\mathrm{MgBr}_{2}\left(4,6-t \mathrm{Bu}-\mathrm{NCOC}_{6} \mathrm{H}_{2}\right)_{2} \mathrm{CH}\right]$ in Benzene 141

ECC- $M W$ Estimation of $\left[\mathrm{Mg}\left(\left(4,6-t \mathrm{Bu}-\mathrm{NCOC}_{6} \mathrm{H}_{2}\right)_{2} \mathrm{CH}\right)_{2}\right]$ in Benzene 141

Molar Van-Der-Waals Densities $\left(M D_{\mathrm{W}}\right) \quad 142$

$\begin{array}{lr}\text { NMR Spectra } & 143\end{array}$

${ }^{1} \mathrm{H}$-DOSY NMR Spectrum of CpLi in Ammonia

${ }^{7} \mathrm{Li},{ }^{1} \mathrm{H}-\mathrm{HOESY}$ NMR Spectrum of CpLi in DMSO 


\section{Categorization of Model Compounds}

Three-dimensional models for reference molecules have been created using the program Avogadro 1.0.3. These can be associated to different molecular shapes (expanded discs (ED), dissipated spheres and ellipsoids (DSE), compact spheres (CS) or rod-like (RL) compounds). The transitions between these shapes are not sharp; still, there are clear systematic trends that can be rationalized. Compact spherical (CS) molecules have nearly the same radius in all dimensions with a highly filled space. Benzene $(78 \mathrm{~g} / \mathrm{mol})$ was found to diffuse CS-like. Dissipated spheres and ellipsoids (DSE) have an elongated major axis and/or a less filled space. Small annelated aromatic compounds like toluene $(92 \mathrm{~g} / \mathrm{mol})$, indene $(116 \mathrm{~g} / \mathrm{mol})$ or naphthalene $(128 \mathrm{~g} / \mathrm{mol})$ with $M W \mathrm{~s}<150 \mathrm{~g} / \mathrm{mol}$ also diffuse DSE-like. Rod-like molecules have long alkyl chains which dominate the overall structure (brominated rod-like molecules have not been listed below). Lastly, enlarged discs are flat molecules, with two elongated major axes and $M W \mathrm{~s}>150 \mathrm{~g} / \mathrm{mol}$.

Table 5-1: Categorization of all model compounds depending to their molecular shapes. Note that in some cases this categorization may be empirical as described above.

\begin{tabular}{|c|c|c|c|c|}
\hline & $\begin{array}{c}\text { Compact spheres } \\
\text { (CS) }\end{array}$ & $\begin{array}{c}\text { Dissipated spheres and } \\
\text { ellipsoids (DSE) }\end{array}$ & Expanded discs (ED) & $\begin{array}{c}\text { Rod-like }(\mathrm{RL}) \\
\text { molecules }\end{array}$ \\
\hline $\begin{array}{c}M W \\
{[\mathrm{~g} / \mathrm{mol}]}\end{array}$ & $\left(M W_{\text {calc }}[\mathrm{g} / \mathrm{mol}]\right)$ & $\left(M W_{\text {calc }}[\mathrm{g} / \mathrm{mol}]\right)$ & $\left(M W_{\text {calc }}[\mathrm{g} / \mathrm{mol}]\right)$ & $\left(M W_{\text {calc }}[\mathrm{g} / \mathrm{mol}]\right)$ \\
\hline \multicolumn{5}{|l|}{$70-80$} \\
\hline & Cyclopentane (70) & & & \\
\hline & THF (72) & & & \\
\hline & Benzene (78) & & & \\
\hline
\end{tabular}

$80-100$

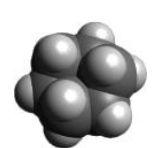

Cyclohexane (84)

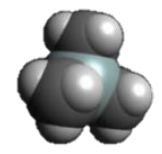

TMS (88)
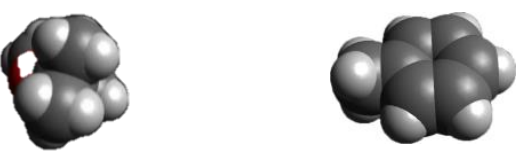

MTBE (88)

Toluene (92) 


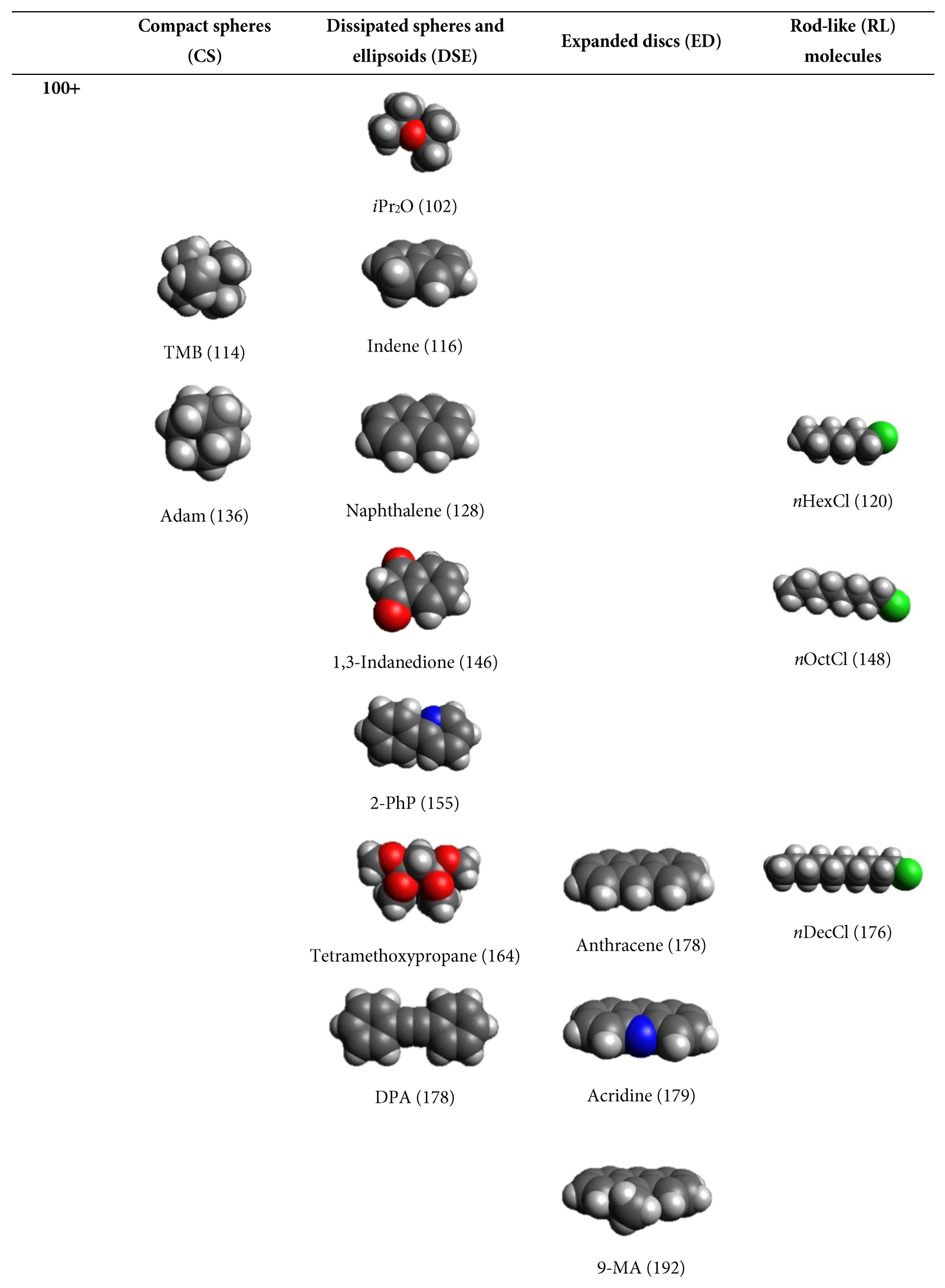




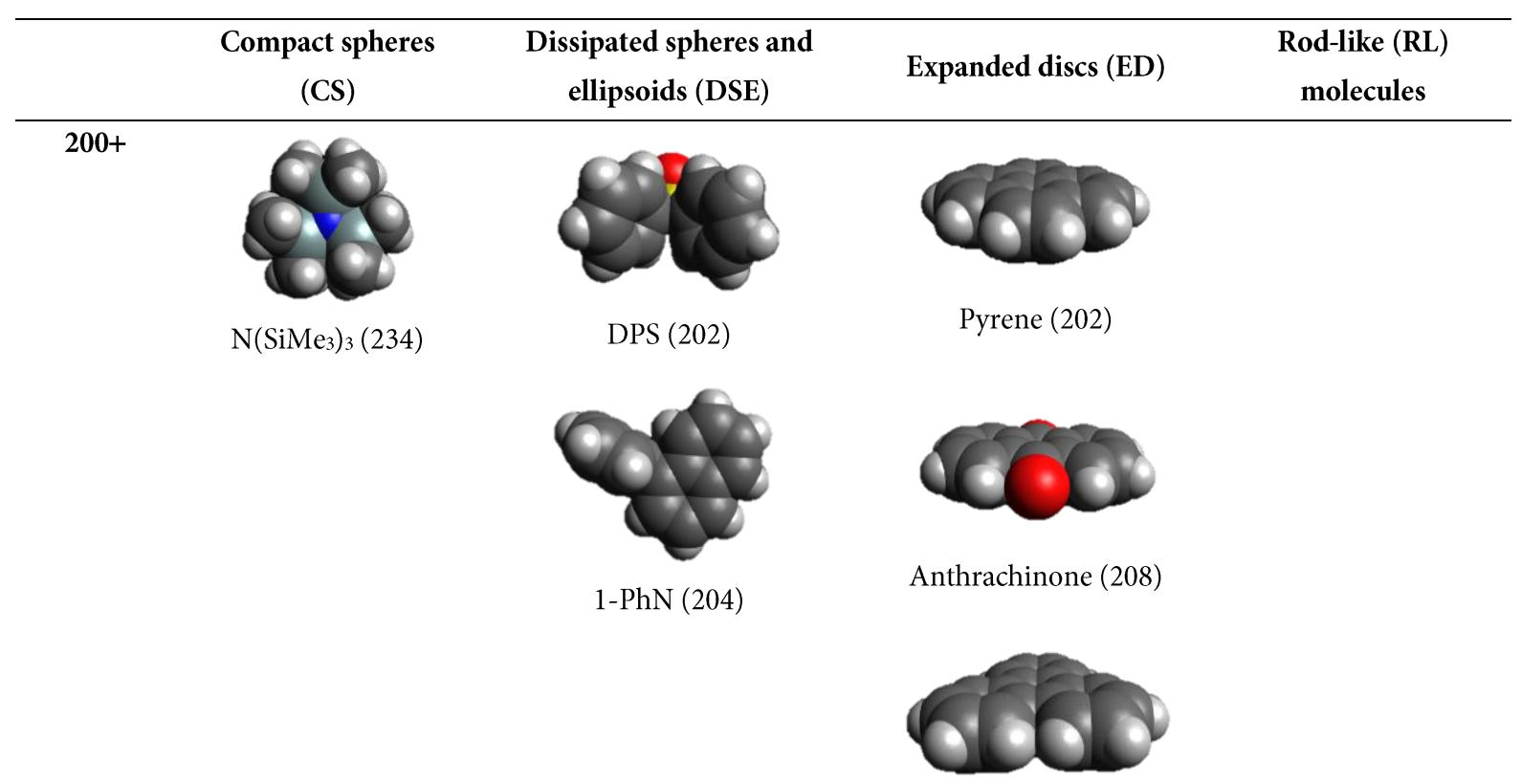

Triphenylene (228)

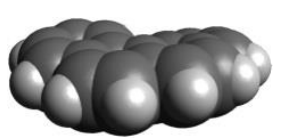

DHBP (281)

$300+$
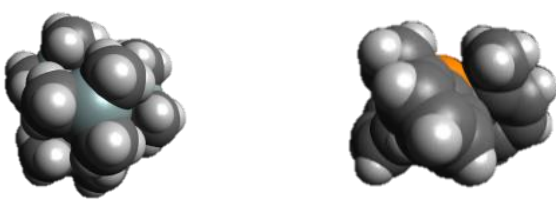

ceservitus

$\mathrm{Si}\left(\mathrm{SiMe}_{3}\right)_{4}(321)$

Tri(otolyl)phosphine (304)

Batylalcohol (345)

$400+$

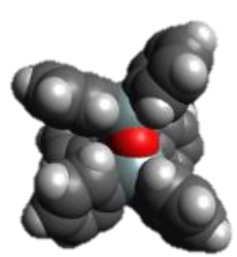

Hexaphenyltrisiloxane

(595)

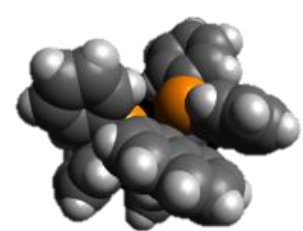

BINAP (623)

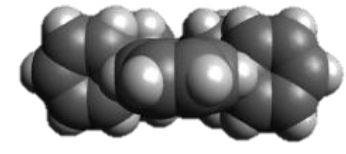

TPhN (433) 


\section{Principal Moments of Inertia of Model Compounds}

For a more theoretical approach towards categorization, principal moments of inertia of model compounds were calculated as described in section 2.1.2 (results and conclusion can also be found in section 2.1.2).

Table 5-2: Principal moments of inertia calculated with PMIFST ${ }^{[122]}$ for geometry optimized (MMFF94) structures of model compounds used in ECCs. The relative shape anisotropy $\kappa^{2}$ is also listed for each model compound.

\begin{tabular}{|c|c|c|c|c|c|}
\hline Compound & $I_{\mathrm{xx}}\left[\mathrm{u} \AA^{2}\right]$ & $\boldsymbol{I}_{\mathrm{yy}}\left[\mathbf{u} \AA^{2}\right]$ & $I_{z z}\left[\mathbf{u} \AA^{2}\right]$ & $\sim I_{\mathrm{xx}}: I_{\mathrm{yy}}: I_{\mathrm{zz}}$ & $\kappa^{2} \cdot 100 \%$ \\
\hline 1,3-Indanedione & 301 & 408 & 707 & $1: 1: 2$ & 6.6 \\
\hline $1-\mathrm{PhN}$ & 473 & 1354 & 1680 & $1: 3: 4$ & 9.5 \\
\hline 2-PhP & 173 & 905 & 1053 & $1: 5: 6$ & 14.7 \\
\hline 9-MA & 356 & 1133 & 1485 & $1: 3: 4$ & 11.3 \\
\hline Acridine & 227 & 1081 & 1304 & $1: 5: 6$ & 14.2 \\
\hline Anthracene & 230 & 1113 & 1343 & $1: 5: 6$ & 14.4 \\
\hline Anthrachinone & 450 & 1142 & 1592 & $1: 3: 4$ & 9.8 \\
\hline Batylalcohol & 274 & 20730 & 20911 & $1: 76: 76$ & 24.0 \\
\hline Benzene & 89 & 89 & 177 & $1: 1: 2$ & 6.1 \\
\hline BINAP & 6783 & 7518 & 9435 & $1: 1: 1$ & 1.0 \\
\hline $\mathrm{CHCl}_{3}$ & 154 & 154 & 296 & $1: 1: 2$ & 5.5 \\
\hline $\mathrm{CH}_{2} \mathrm{Cl}_{2}$ & 16 & 150 & 163 & $1: 9: 10$ & 18.4 \\
\hline $\mathrm{CH}_{3} \mathrm{CN}$ & 3 & 54 & 54 & $1: 18: 18$ & 21.1 \\
\hline $\mathrm{CH}_{3} \mathrm{OH}$ & 4 & 20 & 21 & $1: 5: 5$ & 13.5 \\
\hline$n$ DecCl & 70 & 3349 & 3387 & $1: 48: 48$ & 23.5 \\
\hline DHBP & 1146 & 1890 & 2716 & $1: 2: 2$ & 5.6 \\
\hline Cyclohexane & 117 & 117 & 205 & $1: 1: 2$ & 4.0 \\
\hline Cyclopentane & 76 & 76 & 132 & $1: 1: 3$ & 3.9 \\
\hline DMSO & 73 & 74 & 122 & $1: 1: 2$ & 3.3 \\
\hline DPA & 178 & 2020 & 2200 & $1: 11: 12$ & 19.4 \\
\hline DPS & 426 & 1217 & 1425 & $1: 3: 3$ & 8.9 \\
\hline Hexaphenyltrisiloxane & 6123 & 7683 & 8920 & $1: 1: 1$ & 1.1 \\
\hline$n \mathrm{HexCl}$ & 44 & 956 & 980 & $1: 22: 22$ & 21.8 \\
\hline $\mathrm{H}_{2} \mathrm{O}$ & 0.6 & 1.2 & 1.8 & $1: 2: 3$ & 4.0 \\
\hline Indene & 135 & 313 & 444 & $1: 2: 3$ & 9.1 \\
\hline$i \mathrm{Pr}_{2} \mathrm{O}$ & 122 & 326 & 370 & $1: 3: 3$ & 7.9 \\
\hline MTBE & 115 & 187 & 187 & $1: 2: 2$ & 2.2 \\
\hline Naphthalene & 160 & 409 & 568 & $1: 3: 4$ & 9.8 \\
\hline $\mathrm{N}\left(\mathrm{SiMe}_{3}\right)_{3}$ & 893 & 893 & 1311 & $1: 1: 1$ & 1.8 \\
\hline$n O c t C l$ & 57 & 1916 & 1948 & $1: 34: 34$ & 22.9 \\
\hline Pyrene & 490 & 904 & 1394 & $1: 2: 3$ & 7.9 \\
\hline $\mathrm{Si}(\mathrm{OMe})_{4}$ & 345 & 412 & 494 & $1: 1: 1$ & 1.1 \\
\hline $\mathrm{Si}\left(\mathrm{SiMe}_{3}\right)_{4}$ & 1914 & 1914 & 1914 & $1: 1: 1$ & 0 \\
\hline Tetramethoxypropane & 97 & 318 & 391 & $1: 3: 4$ & 10.8 \\
\hline $\mathrm{TPhN}$ & 2876 & 4349 & 6524 & $1: 2: 2$ & 5.4 \\
\hline THF & 70 & 72 & 126 & $1: 1: 2$ & 4.2 \\
\hline TMB & 219 & 510 & 515 & $1: 2: 2$ & 5.6 \\
\hline TMS & 162 & 162 & 162 & $1: 1: 1$ & 0 \\
\hline Toluene & 92 & 199 & 288 & $1: 2: 3$ & 8.6 \\
\hline Tri(otolyl)phosphine & 1790 & 1883 & 2840 & $1: 1: 2$ & 2.4 \\
\hline Triphenylene & 990 & 990 & 1980 & $1: 1: 2$ & 6.3 \\
\hline Triphenylmethane & 1372 & 1331 & 2271 & $1: 1: 2$ & 3.4 \\
\hline
\end{tabular}




\section{Absolute Logarithmic Diffusion Coefficients of Model Compounds for Various Solvents}

Table 5-3: Exemplary absolute logarithmic diffusion coefficients $\left(\log \left(D_{\mathrm{x}}\right)\right.$; not normalized) of model compounds and solvents (rod-like references used in $\mathrm{ECC}^{\mathrm{THF}}$ are not listed) at $25^{\circ} \mathrm{C}$. Due to solubility problems not all references could be measured in all solvents (marked with ${ }^{\star}$ ). There are also references not considered in calibration curves due to unknown aggregational behavior or elevated molar van-der-Waals densities (marked in red).

\begin{tabular}{|c|c|c|c|c|c|c|c|}
\hline Solvent & DMSO & $\mathrm{C}_{6} \mathrm{D}_{12}$ & $\mathrm{CDCl}_{3}$ & $\mathrm{CD}_{3} \mathrm{OD}$ & $\mathrm{C}_{6} \mathrm{D}_{6}$ & $\mathrm{CD}_{2} \mathrm{Cl}_{2}$ & $\mathrm{CD}_{3} \mathrm{CN}$ \\
\hline Compound & $\log \left(D_{\mathrm{x}}\right)$ & $\log \left(D_{\mathrm{x}}\right)$ & $\log \left(D_{\mathrm{x}}\right)$ & $\log \left(D_{\mathrm{x}}\right)$ & $\log \left(D_{\mathrm{x}}\right)$ & $\log \left(D_{\mathrm{x}}\right)$ & $\log \left(D_{\mathrm{x}}\right)$ \\
\hline 1,3-Indanedione & * & -8.9258 & -8.8558 & -8.8496 & -8.7970 & -8.7172 & -8.6146 \\
\hline 1-PhN & * & -9.0487 & -8.9019 & -8.8757 & -8.9027 & -8.7908 & -8.7908 \\
\hline 2-PhP & -9.3307 & -8.9081 & -8.8359 & -8.8606 & -8.8189 & -8.7126 & -8.6121 \\
\hline 9-MA & -9.3797 & -8.9890 & -8.8456 & -8.8375 & -8.8546 & -8.7354 & -8.6062 \\
\hline Acridine & -9.3672 & -8.9643 & -8.8644 & -8.9049 & -8.8449 & -8.7363 & -8.6248 \\
\hline Adam & -9.3590 & -9.0204 & -8.8155 & -8.8005 & -8.8077 & -8.6997 & -8.5603 \\
\hline Anthracene & * & -8.9240 & -8.8043 & -8.8047 & -8.8432 & -8.6929 & -8.5502 \\
\hline Anthrachinone & -9.4126 & * & -8.8779 & -8.8575 & -8.8981 & -8.7521 & -8.6566 \\
\hline BINAP & * & -9.3629 & -9.1735 & * & -9.1941 & -9.0582 & * \\
\hline $\mathrm{C}_{6} \mathrm{D}_{6}{ }^{\mathrm{b}}$ & - & - & - & - & -8.6882 & - & - \\
\hline $\mathrm{CDCl}_{3}{ }^{\mathrm{b}}$ & - & - & -8.6519 & - & - & - & - \\
\hline $\mathrm{CD}_{2} \mathrm{Cl}_{2}{ }^{\mathrm{b}}$ & - & - & - & - & - & -8.4593 & - \\
\hline $\mathrm{CD}_{3} \mathrm{CN}^{\mathrm{b}}$ & - & - & - & - & - & - & -8.3765 \\
\hline $\mathrm{CD}_{3} \mathrm{OD}^{\mathrm{b}}$ & - & - & - & -8.6882 & - & - & - \\
\hline Cyclohexane- $d_{12}{ }^{\mathrm{b}}$ & - & -8.8821 & - & - & - & - & - \\
\hline Cyclopentane & -9.1184 & -8.8119 & -8.6459 & -8.6216 & -8.6192 & -8.5411 & -8.4043 \\
\hline DHBP & -9.5327 & -9.1465 & -8.9801 & -8.9709 & -8.9765 & -8.8638 & -8.7340 \\
\hline DPA & -9.3779 & -8.9987 & -8.8573 & -8.8528 & -8.8731 & -8.7495 & -8.6325 \\
\hline DPS & -9.4121 & -9.0473 & -8.9320 & -8.9320 & -8.8972 & -8.7987 & -8.6826 \\
\hline DMSO- $d_{6}{ }^{\mathrm{b}}$ & -9.1896 & - & - & - & - & - & - \\
\hline Hexaphenyltrisiloxane & -9.7354 & -9.3500 & -9.1586 & -9.2094 & -9.1963 & -9.0484 & -8.9409 \\
\hline Indene & -9.2499 & -8.8743 & -8.7479 & -8.7439 & -8.7649 & -8.6424 & -8.5318 \\
\hline$i \operatorname{Pr}_{2} \mathrm{O}$ & -9.1983 & -8.8708 & -8.7747 & -8.7655 & -8.7087 & -8.6505 & -8.5143 \\
\hline MTBE & -9.2022 & -8.8634 & -8.6993 & -8.7143 & -8.7379 & -8.6039 & -8.4930 \\
\hline Naphthalene & -9.2642 & -8.8531 & -8.7086 & -8.7351 & -8.7566 & -8.6336 & -8.5261 \\
\hline $\mathbf{N}\left(\mathrm{SiMe}_{3}\right)_{3}$ & -9.4419 & -9.1007 & -8.9212 & -8.9136 & -8.8993 & -8.8179 & -8.6749 \\
\hline Pyrene & -9.3946 & -8.9644 & -8.8427 & -8.8806 & -8.8814 & -8.7349 & -8.6437 \\
\hline $\mathrm{Si}(\mathrm{OMe})_{4}$ & -9.3038 & -8.9136 & -8.8156 & -8.7991 & -8.7833 & -8.7100 & -8.5891 \\
\hline $\mathrm{Si}\left(\mathrm{SiMe}_{3}\right)_{4}$ & -9.5361 & -9.1894 & -8.9590 & -9.0094 & -8.9731 & -8.8697 & -8.6910 \\
\hline Tetramethoxypropane & -9.3422 & -8.9820 & -8.9008 & -8.8557 & -8.8516 & -8.7650 & -8.6476 \\
\hline $\mathrm{TPhN}$ & -9.7006 & -9.3511 & -9.0567 & -9.1041 & -9.1406 & -8.9472 & -8.8724 \\
\hline THF & -9.1025 & -8.7807 & -8.6826 & -8.6422 & -8.6882 & -8.5599 & -8.4276 \\
\hline TMB & -9.2920 & -8.9338 & -8.7886 & -8.7706 & -8.7886 & -8.6751 & -8.5530 \\
\hline TMS & -9.2324 & -8.8765 & -8.7268 & -8.7082 & -8.7104 & -8.6300 & -8.4808 \\
\hline Toluene & -9.1525 & -8.7901 & -8.6849 & -8.6611 & -8.6842 & -8.5885 & -8.4654 \\
\hline Tri(otolyl)phosphine & -9.5430 & -9.2102 & -9.0419 & -9.0188 & -9.0176 & -8.9121 & -8.7768 \\
\hline Triphenylene & -9.4481 & -9.0358 & -8.8916 & -8.9003 & -8.9361 & -8.7605 & -8.6642 \\
\hline Triphenylmethane & -9.5039 & -9.1407 & -8.9714 & -8.9617 & -8.9794 & -8.8593 & -8.7368 \\
\hline Water & - & - & - & -8.6893 & - & - & -8.2326 \\
\hline
\end{tabular}

${ }^{\text {a }}$ For most model compounds, multiple diffusion coefficients have been recorded which varied to some degree before normalization (temperature or concentration effects).

${ }^{\mathrm{b}}$ Residual solvent signal. 


\section{ECCs and Normalized Diffusion Coefficients of Model Compounds for Various Solvents}

ECCs were established using normalized diffusion coefficients $\left(\log \left(D_{\mathrm{x}, \mathrm{norm}}\right)\right)$ derived with the approaches and formulae discussed in chapter 2.1. The maximum deviation of $\log \left(D_{\mathrm{x}, \mathrm{norm}}\right)$ was 0.0075. $\Delta \log \left(D_{x, \text { norm }}\right)$ was defined as twice this deviation as represented by the error bars in the ECCs. For solvents and internal references (Adam, TMB) with more than 5 separate measurements the error bars represent standard deviations of their specific $\log \left(D_{\mathrm{x}, \mathrm{norm}}\right)$. Normalized diffusion coefficients and estimated MWs may differ slightly from values given in literature: ${ }^{[2-3]}$ There have been on the one hand new insights into the ECC-DOSY methodology and on the other MWs were calculated using values in accordance with significance (for further information see sections 2.1.1 and 2.3.1). 
ECCs and Model Compounds for DMSO- $d_{6}$
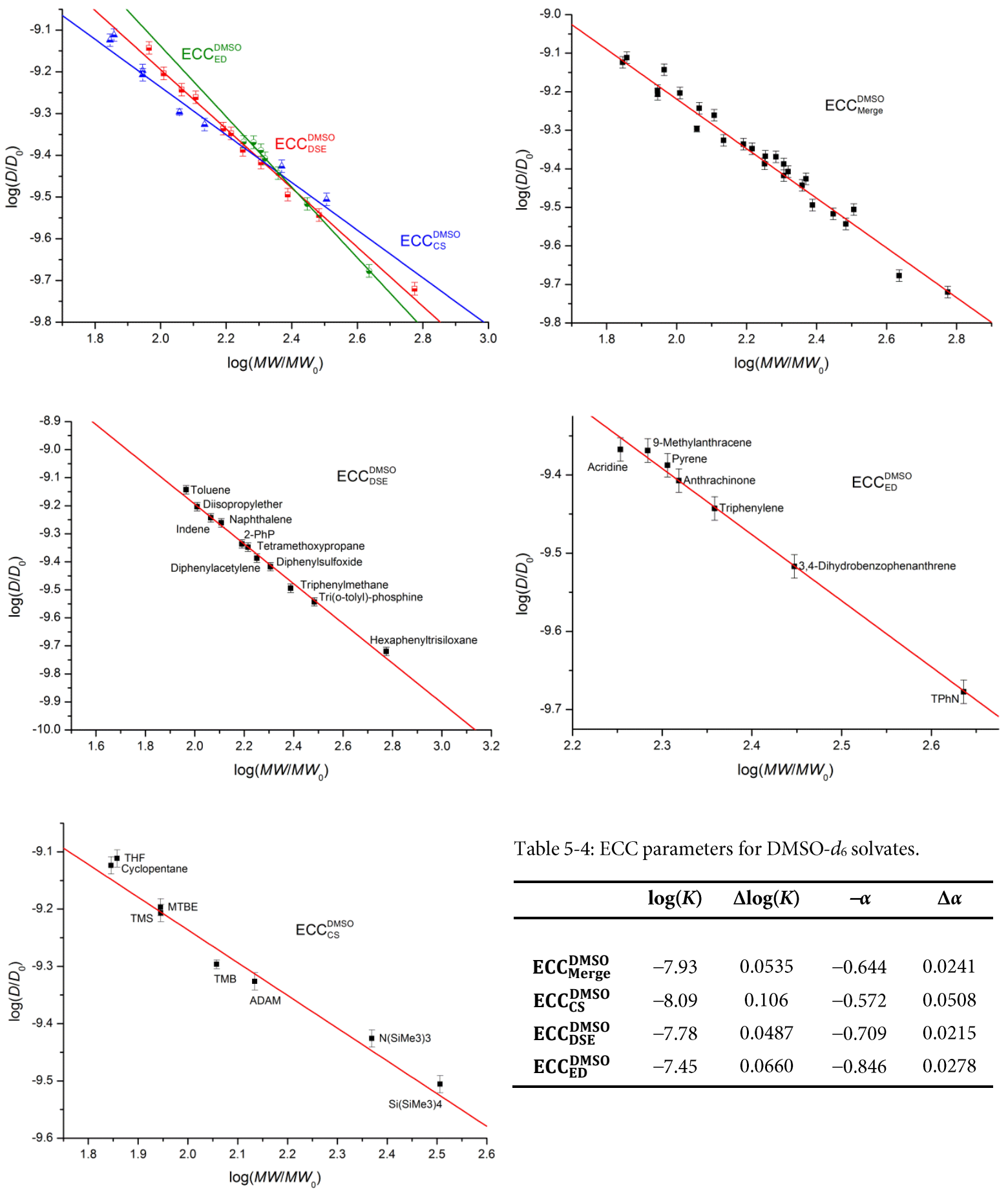

Table 5-4: ECC parameters for DMSO- $d_{6}$ solvates.

\begin{tabular}{lcccc}
\hline & $\log (K)$ & $\Delta \log (K)$ & $-\alpha$ & $\Delta \boldsymbol{\alpha}$ \\
\hline & & & & \\
ECC $_{\text {Merge }}^{\text {DMSO }}$ & -7.93 & 0.0535 & -0.644 & 0.0241 \\
ECC CS $_{\text {CS }}^{\text {DMSO }}$ & -8.09 & 0.106 & -0.572 & 0.0508 \\
ECC $_{\text {DSE }}^{\text {DMSO }}$ & -7.78 & 0.0487 & -0.709 & 0.0215 \\
ECC $_{\text {ED }}^{\text {DMSO }}$ & -7.45 & 0.0660 & -0.846 & 0.0278 \\
\hline
\end{tabular}

Figure 5-1: Plots of $\log \left(D_{\mathrm{x}, \mathrm{norm}}\right)$ vs. $\log \left(M W_{\text {calc }}\right)$ of all used model compounds in DMSO- $d_{6}$ sorted by their molecular shape. There is also a merged ECC of all measured compounds for $M W$ estimation, when the molecular shape of an analyte is unknown. 
Table 5-5: Overview of all used model compounds for ECC ${ }^{\text {DMSO }}$ and their normalized diffusion coefficients $\log \left(D_{\mathrm{x}, \mathrm{norm}}\right)$, the estimated $M W_{\text {det }}$ and the deviation from the calculated molecular weight $M W_{\text {dif. }}$ TMB was used as internal reference with $\log \left(D_{\text {reffix }}\right)(\mathrm{TMB})=-9.2920$. All compounds have been measured as $15 \mathrm{mM}$ solutions of analyte and TMB in an equimolar ratio. ${ }^{\mathrm{a}}$

\begin{tabular}{|c|c|c|c|c|c|c|}
\hline $\begin{array}{c}M W \\
{[\mathrm{~g} / \mathrm{mol}]}\end{array}$ & & $\begin{array}{l}D_{\mathbf{x}, \text { norm }} \\
{\left[\mathbf{m}^{2} / \mathbf{s}\right]}\end{array}$ & $\begin{array}{c}\log \\
\left(D_{\mathrm{x}, \text { norm }}\right)\end{array}$ & $\begin{array}{c}\log \\
\left(M W_{\text {det }}\right)\end{array}$ & $\begin{array}{c}M W_{\text {det }} \\
{[\mathrm{g} / \mathrm{mol}]}\end{array}$ & $\begin{array}{c}M W_{\text {dif }} \\
{[\%]}\end{array}$ \\
\hline & Not assigned & & & & & \\
\hline 78 & $\mathrm{DMSO}^{\mathrm{b}}$ & $6.6267 \mathrm{E}-10$ & -9.1787 & - & - & - \\
\hline \multirow[t]{2}{*}{152} & $\mathrm{Si}(\mathrm{OMe})_{4}^{\mathrm{b}}$ & $5.2977 \mathrm{E}-10$ & -9.2759 & - & - & - \\
\hline & Compact spheres, $E_{C C}$ & & & & & \\
\hline 70 & Cyclopentane & $7.5204 \mathrm{E}-10$ & -9.1238 & 1.81 & 64 & 9 \\
\hline 72 & THF & $7.7359 \mathrm{E}-10$ & -9.1115 & 1.79 & 61 & 18 \\
\hline 88 & MTBE & $6.3534 \mathrm{E}-10$ & -9.1970 & 1.94 & 86 & 2 \\
\hline 88 & TMS & $6.2554 \mathrm{E}-10$ & -9.2037 & 1.95 & 89 & 0 \\
\hline 114 & TMB & $5.0548 \mathrm{E}-10$ & -9.2963 & 2.11 & 129 & -11 \\
\hline 136 & Adam & $4.7181 \mathrm{E}-10$ & -9.3262 & 2.16 & 145 & -6 \\
\hline 234 & $\mathrm{~N}\left(\mathrm{SiMe}_{3}\right)_{3}$ & $3.7498 \mathrm{E}-09$ & -9.4260 & 2.34 & 217 & 8 \\
\hline \multirow[t]{2}{*}{321} & $\mathrm{Si}\left(\mathrm{SiMe}_{3}\right)_{4}$ & $3.1204 \mathrm{E}-10$ & -9.5058 & 2.48 & 299 & 7 \\
\hline & Dissipated spheres \& ellipsoids, ECC & & & & & \\
\hline 92 & Toluene & $7.1967 \mathrm{E}-10$ & -9.1423 & 1.92 & 84 & 10 \\
\hline 102 & $i \mathrm{Pr}_{2} \mathrm{O}$ & $6.2574 \mathrm{E}-10$ & -9.2036 & 2.01 & 102 & 0 \\
\hline 116 & Indene & $5.7155 \mathrm{E}-10$ & -9.2429 & 2.06 & 116 & 0 \\
\hline 128 & Naphthalene & $5.4835 \mathrm{E}-10$ & -9.2609 & 2.09 & 123 & 4 \\
\hline 155 & 2-PhP & $4.6127 \mathrm{E}-10$ & -9.3360 & 2.19 & 157 & -1 \\
\hline 164 & Tetramethoxypropane & $4.4923 \mathrm{E}-10$ & -9.3475 & 2.21 & 163 & 1 \\
\hline 178 & DPA & $4.1031 \mathrm{E}-10$ & -9.3869 & 2.27 & 185 & -3 \\
\hline 202 & DPS & $3.8249 \mathrm{E}-10$ & -9.4174 & 2.31 & 204 & -1 \\
\hline 244 & Triphenylmethane & $3.2045 \mathrm{E}-10$ & -9.4942 & 2.42 & 262 & -7 \\
\hline 304 & Tri(otolyl)phosphine & $2.8624 \mathrm{E}-10$ & -9.5433 & 2.49 & 307 & -1 \\
\hline \multirow[t]{2}{*}{595} & Hexaphenyltrisiloxane & $1.9064 \mathrm{E}-10$ & -9.7198 & 2.74 & 544 & 9 \\
\hline & Expanded discs, $E C C_{E D}$ & & & & & \\
\hline 179 & Acridine & $4.2907 \mathrm{E}-10$ & -9.3675 & 2.27 & 185 & -3 \\
\hline 192 & 9-MA & $4.2782 \mathrm{E}-10$ & -9.3687 & 2.27 & 185 & 4 \\
\hline 202 & Pyrene & $4.0960 \mathrm{E}-10$ & -9.3876 & 2.29 & 195 & 4 \\
\hline 208 & Anthrachinone & $3.9145 \mathrm{E}-10$ & -9.4073 & 2.31 & 206 & 1 \\
\hline 228 & Triphenylene & $3.6074 \mathrm{E}-10$ & -9.4428 & 2.36 & 227 & 1 \\
\hline 281 & DHBP & $3.0426 \mathrm{E}-10$ & -9.5168 & 2.45 & 282 & 1 \\
\hline \multirow[t]{2}{*}{433} & TPhN & $2.1029 \mathrm{E}-10$ & -9.6772 & 2.63 & 429 & 1 \\
\hline & & & & & $\varnothing$ & \pm 4 \\
\hline
\end{tabular}

${ }^{a}$ When a compound had more than one resonance in the ${ }^{1} \mathrm{H}$ NMR spectrum, the average diffusion coefficient of all resonances was used.

${ }^{\mathrm{b}} \mathrm{DMSO}$ and $\mathrm{Si}(\mathrm{OMe})_{4}$ were excluded due to elevated molar van-der-Waals density (see Table 5-24). 


\section{ECCs and Model Compounds for DMSO- $d_{6}$ at Different Temperatures (Compact Spherical Molecules)}

Table 5-6: Overview of compact spherical (CS) model compounds for ECC $^{\text {DMSO }}$ and their normalized diffusion coefficients $\log \left(D_{\mathrm{x}, \text { norm }}\right)$ measured at different temperatures $\left(25^{\circ} \mathrm{C}\right.$ to $\left.60^{\circ} \mathrm{C}\right)$. TMB was used as internal reference with $\log \left(D_{\text {reffix }}\right)(\mathrm{TMB})=-9.2920$. All compounds have been measured as $15 \mathrm{mM}$ solutions of analyte and TMB in an equimolar ratio. ${ }^{\mathrm{a}}$

\begin{tabular}{|c|c|c|c|c|c|c|}
\hline $\begin{array}{c}M W \\
{[\mathrm{~g} / \mathrm{mol}]}\end{array}$ & & $\begin{array}{c}\log \\
\left(D_{\mathrm{x}, \text { norm }}\right)\end{array}$ & $\begin{array}{c}\log \\
\left(D_{\mathrm{x}, \text { norm }}\right)\end{array}$ & $\begin{array}{c}\log \\
\left(D_{\mathrm{x}, \text { norm }}\right) \\
\end{array}$ & $\begin{array}{c}\log \\
\left(D_{\mathrm{x}, \text { norm }}\right)\end{array}$ & $\begin{array}{c}\log \\
\left(D_{\mathrm{x}, \text { norm }}\right) \\
\end{array}$ \\
\hline & Temperature & $25^{\circ} \mathrm{C}$ & $30{ }^{\circ} \mathrm{C}$ & $40{ }^{\circ} \mathrm{C}$ & $50^{\circ} \mathrm{C}$ & $60{ }^{\circ} \mathrm{C}$ \\
\hline 70 & Cyclopentane & -9.1238 & -9.1324 & -9.1321 & -9.1492 & -9.1430 \\
\hline 72 & THF & -9.1115 & -9.1103 & -9.1155 & -9.1255 & -9.1250 \\
\hline 88 & MTBE & -9.1970 & -9.1999 & -9.2021 & -9.2030 & -9.2094 \\
\hline 88 & TMS & -9.2037 & -9.2146 & -9.2163 & -9.2225 & -9.2271 \\
\hline 136 & Adam & -9.3262 & -9.3261 & -9.3221 & -9.3430 & -9.3283 \\
\hline 234 & $\mathrm{~N}\left(\mathrm{SiMe}_{3}\right)_{3}$ & -9.4260 & -9.4227 & -9.4250 & -9.4249 & -9.4160 \\
\hline 321 & $\mathrm{Si}\left(\mathrm{SiMe}_{3}\right)_{4}$ & -9.5058 & -9.4970 & -9.4937 & -9.4898 & -9.5210 \\
\hline
\end{tabular}

Table 5-7: ECC parameters for compact spherical solvates in DMSO- $d_{6}$ at different temperatures (plotted without TMB).

\begin{tabular}{cccccc}
\hline Temperature & $\log (\boldsymbol{K})$ & $\Delta \log (\boldsymbol{K})$ & $-\boldsymbol{\alpha}$ & $\boldsymbol{\Delta \alpha}$ & ${\text { cor. } \mathrm{R}^{\mathbf{2}}}$ \\
& & & & & \\
$\mathbf{2 5}{ }^{\circ} \mathrm{C}$ & -8.06 & 0.0650 & -0.580 & 0.0310 & 0.98 \\
$\mathbf{3 0}{ }^{\circ} \mathrm{C}$ & -8.10 & 0.0745 & -0.559 & 0.0355 & 0.98 \\
$\mathbf{4 0}{ }^{\circ} \mathrm{C}$ & -8.12 & 0.0687 & -0.553 & 0.0327 & 0.98 \\
$\mathbf{5 0}{ }^{\circ} \mathrm{C}$ & -8.17 & 0.0807 & -0.533 & 0.0384 & 0.97 \\
$\mathbf{6 0}{ }^{\circ} \mathrm{C}$ & -8.12 & 0.0729 & -0.558 & 0.0347 & 0.98 \\
& & & & & \\
\hline
\end{tabular}

${ }^{a}$ When a compound had more than one resonance in the ${ }^{1} \mathrm{H}$ NMR spectrum, the average diffusion coefficient of all resonances was used. 
ECCs and Model Compounds for Cyclohexane- $d_{12}$
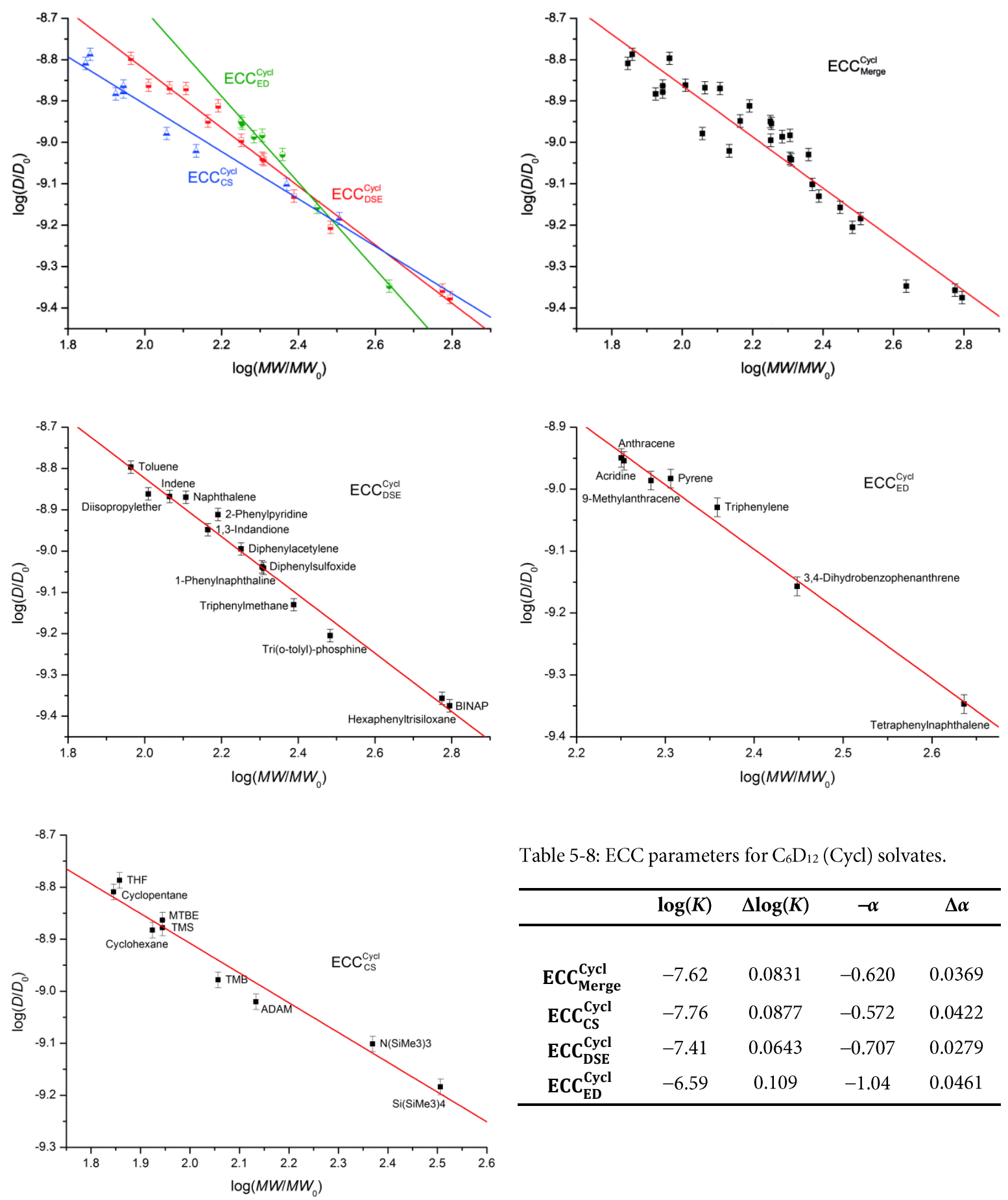

Table 5-8: ECC parameters for $\mathrm{C}_{6} \mathrm{D}_{12}$ (Cycl) solvates.

\begin{tabular}{lcccc}
\hline & $\log (K)$ & $\Delta \log (K)$ & $-\alpha$ & $\Delta \alpha$ \\
\hline & & & & \\
ECC $_{\text {Merge }}^{\text {Cycl }}$ & -7.62 & 0.0831 & -0.620 & 0.0369 \\
ECC $_{\text {CS }}^{\text {Cycl }}$ & -7.76 & 0.0877 & -0.572 & 0.0422 \\
ECC CSE $_{\text {DSE }}^{\text {Cycl }}$ & -7.41 & 0.0643 & -0.707 & 0.0279 \\
ECC $_{\text {ED }}^{\text {Cycl }}$ & -6.59 & 0.109 & -1.04 & 0.0461 \\
\hline
\end{tabular}

Figure 5-2: Plots of $\log \left(D_{\mathrm{x}, \mathrm{norm}}\right)$ vs. $\log \left(M W_{\text {calc }}\right)$ of all used model compounds in $\mathrm{C}_{6} \mathrm{D}_{12}(\mathrm{Cycl})$ sorted by their molecular shape. There is also a merged ECC of all measured compounds for $M W$ estimation, when the molecular shape of an analyte is unknown. 
Table 5-9: Overview of all used model compounds for ECC Cycl and their normalized diffusion coefficients $\log \left(D_{\mathrm{x}, \text { norm }}\right)$, the

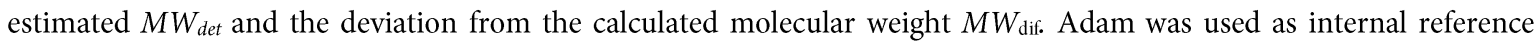
with $\log \left(D_{\text {reffix }}\right)($ Adam $)=-9.0204$. All compounds have been measured as $15 \mathrm{~mm}$ solutions of analyte and Adam in an equimolar ratio. ${ }^{\mathrm{a}}$

\begin{tabular}{|c|c|c|c|c|c|c|}
\hline $\begin{array}{c}M W \\
{[\mathrm{~g} / \mathrm{mol}]}\end{array}$ & & $\begin{array}{l}D_{\mathbf{x}, \text { norm }} \\
{\left[\mathbf{m}^{2} / \mathbf{s}\right]}\end{array}$ & $\begin{array}{c}\log \\
\left(D_{x, \text { norm }}\right) \\
\end{array}$ & $\begin{array}{c}\log \\
\left(M W_{\text {det }}\right)\end{array}$ & $\begin{array}{c}M W_{\text {det }} \\
{[\mathrm{g} / \mathrm{mol}]}\end{array}$ & $\begin{array}{c}M W_{\text {dif }} \\
{[\%]}\end{array}$ \\
\hline & Not assigned & & & & & \\
\hline \multirow[t]{2}{*}{78} & $\mathrm{Si}(\mathrm{OMe})_{4}^{\mathrm{b}}$ & $1.2321 \mathrm{E}-10$ & -8.9093 & - & - & - \\
\hline & Compact spheres, ECCCS & & & & & \\
\hline 70 & Cyclopentane & $1.5519 \mathrm{E}-09$ & -8.8091 & 1.83 & 68 & 3 \\
\hline 72 & THF & $1.6324 \mathrm{E}-09$ & -8.7872 & 1.80 & 62 & 15 \\
\hline 84 & Cyclohexane & $1.3101 \mathrm{E}-09$ & -8.8827 & 1.96 & 92 & -8 \\
\hline 88 & MTBE & $1.3695 \mathrm{E}-09$ & -8.8634 & 1.93 & 85 & 4 \\
\hline 88 & TMS & $1.3240 \mathrm{E}-09$ & -8.8781 & 1.95 & 90 & -2 \\
\hline 114 & TMB & $1.0512 \mathrm{E}-09$ & -8.9783 & 2.13 & 135 & -15 \\
\hline 136 & Adam & $9.5422 \mathrm{E}-10$ & -9.0204 & 2.20 & 160 & -15 \\
\hline 234 & $\mathrm{~N}\left(\mathrm{SiMe}_{3}\right)_{3}$ & $7.9187 \mathrm{E}-10$ & -9.1013 & 2.35 & 221 & 6 \\
\hline \multirow[t]{2}{*}{321} & $\mathrm{Si}\left(\mathrm{SiMe}_{3}\right)_{4}$ & $6.5485 \mathrm{E}-10$ & -9.1839 & 2.49 & 309 & 4 \\
\hline & Dissipated spheres \& ellipsoids, ECC & & & & & \\
\hline 92 & Toluene & $1.5974 \mathrm{E}-09$ & -8.7966 & 1.96 & 91 & 1 \\
\hline 102 & $i \mathrm{Pr}_{2} \mathrm{O}$ & $1.3754 \mathrm{E}-09$ & -8.8616 & 2.05 & 113 & -10 \\
\hline 116 & Indene & $1.3555 \mathrm{E}-09$ & -8.8679 & 2.06 & 115 & 1 \\
\hline 128 & Naphthalene & $1.3500 \mathrm{E}-09$ & -8.8697 & 2.06 & 116 & 10 \\
\hline 146 & 1,3-Indanedione & $1.1227 \mathrm{E}-09$ & -8.9484 & 2.18 & 150 & -3 \\
\hline 155 & 2-PhP & $1.2257 \mathrm{E}-09$ & -8.9116 & 2.12 & 133 & 17 \\
\hline 164 & Tetramethoxypropane & $1.0685 \mathrm{E}-09$ & -8.9712 & 2.21 & 162 & 2 \\
\hline 178 & DPA & $1.0119 \mathrm{E}-09$ & -8.9949 & 2.24 & 174 & 2 \\
\hline 202 & DPS & $9.1607 \mathrm{E}-10$ & -9.0381 & 2.30 & 201 & 1 \\
\hline 204 & $1-\mathrm{PhN}$ & $9.0830 \mathrm{E}-10$ & -9.0418 & 2.31 & 203 & 0 \\
\hline 244 & Triphenylmethane & $7.4143 \mathrm{E}-10$ & -9.1299 & 2.43 & 271 & -10 \\
\hline 304 & Tri(otolyl)phosphine & $6.2356 \mathrm{E}-10$ & -9.2051 & 2.54 & 346 & -12 \\
\hline 595 & Hexaphenyltrisiloxane & $4.3976 \mathrm{E}-10$ & -9.3568 & 2.75 & 567 & 5 \\
\hline \multirow[t]{2}{*}{623} & BINAP & $4.2189 \mathrm{E}-10$ & -9.3749 & 2.78 & 601 & 4 \\
\hline & Expanded discs, $E C C_{E D}$ & & & & & \\
\hline 178 & Anthracene & $1.1232 \mathrm{E}-09$ & -8.9495 & 2.27 & 186 & -4 \\
\hline 179 & Acridine & $1.1110 \mathrm{E}-09$ & -8.9543 & 2.27 & 188 & -5 \\
\hline 192 & 9-MA & $1.0323 \mathrm{E}-09$ & -8.9862 & 2.30 & 201 & -5 \\
\hline 202 & Pyrene & $1.0399 \mathrm{E}-09$ & -8.9830 & 2.30 & 200 & 1 \\
\hline 228 & Triphenylene & $9.3458 \mathrm{E}-10$ & -9.0294 & 2.35 & 222 & 3 \\
\hline 281 & DHBP & $6.9666 \mathrm{E}-10$ & -9.1570 & 2.47 & 294 & -4 \\
\hline \multirow[t]{2}{*}{433} & TPhN & $4.4971 \mathrm{E}-10$ & -9.3471 & 2.65 & 448 & -3 \\
\hline & & & & & $\varnothing$ & \pm 5 \\
\hline
\end{tabular}

\footnotetext{
a When a compound had more than one resonance in the ${ }^{1} \mathrm{H}$ NMR spectrum, the average diffusion coefficient of all resonances was used.

${ }^{\mathrm{b}} \mathrm{Si}(\mathrm{OMe})_{4}$ was excluded due to elevated molar van-der-Waals density (see Table 5-24).
} 
ECCs and Model Compounds for $\mathrm{CDCl}_{3}$
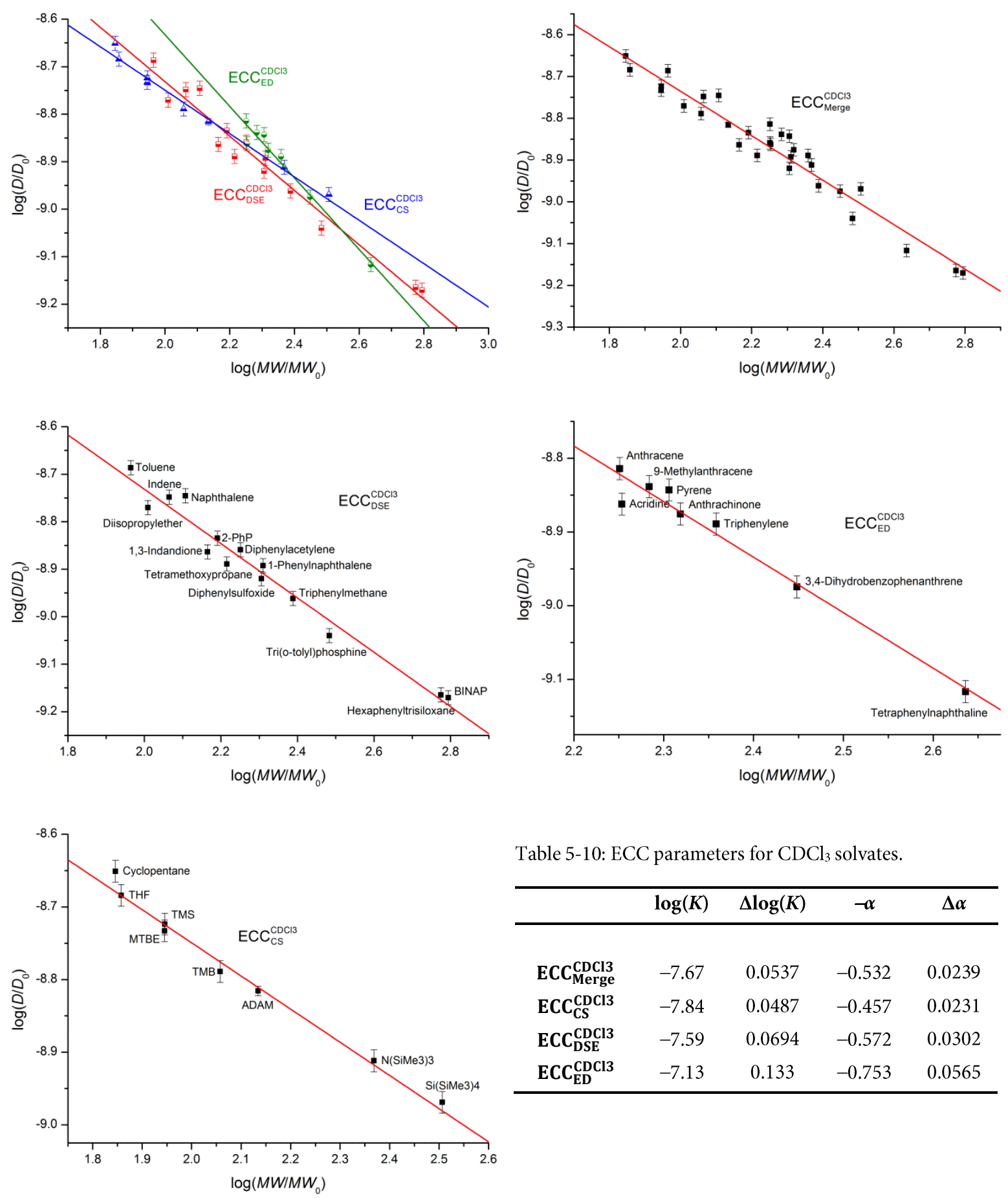

Table 5-10: ECC parameters for $\mathrm{CDCl}_{3}$ solvates.

\begin{tabular}{lcccc}
\hline & $\log (K)$ & $\Delta \log (K)$ & $-\alpha$ & $\Delta \alpha$ \\
\hline & & & & \\
ECC CDerge $_{\text {CDC13 }}^{\text {CDC }}$ & -7.67 & 0.0537 & -0.532 & 0.0239 \\
ECC CS $_{\text {CS }}^{\text {DCl3 }}$ & -7.84 & 0.0487 & -0.457 & 0.0231 \\
ECC $_{\mathbf{D S E}}^{\text {CDCl3 }}$ & -7.59 & 0.0694 & -0.572 & 0.0302 \\
ECC CD $_{\text {ED }}^{\text {CDCl3 }}$ & -7.13 & 0.133 & -0.753 & 0.0565 \\
\hline
\end{tabular}

Figure 5-3: Plots of $\log \left(D_{\mathrm{x}, \mathrm{norm}}\right)$ vs. $\log \left(M W_{\text {calc }}\right)$ of all used model compounds in $\mathrm{CDCl}_{3}$ sorted by their molecular shape. There is also a merged ECC of all measured compounds for $M W$ estimation, when the molecular shape of an analyte is unknown. 
Table 5-11: Overview of all used model compounds for ECC $\mathrm{CDCl}^{3}$ and their normalized diffusion coefficients $\log \left(D_{\mathrm{x}, \mathrm{norm}}\right)$,

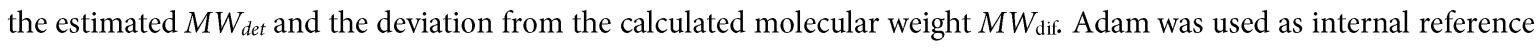
with $\log \left(D_{\text {reffix }}\right)($ Adam $)=-8.8155$. All compounds have been measured as $15 \mathrm{~mm}$ solutions of analyte and Adam in an equimolar ratio. ${ }^{\mathrm{a}}$

\begin{tabular}{|c|c|c|c|c|c|c|}
\hline $\begin{array}{c}M W \\
{[\mathrm{~g} / \mathrm{mol}]}\end{array}$ & & $\begin{array}{l}D_{\mathrm{x}, \text { norm }} \\
{\left[\mathbf{m}^{2} / \mathbf{s}\right]}\end{array}$ & $\begin{array}{c}\log \\
\left(D_{x, \text { norm }}\right) \\
\end{array}$ & $\begin{array}{c}\log \\
\left(M W_{\text {det }}\right)\end{array}$ & $\begin{array}{c}M W_{\text {det }} \\
{[\mathrm{g} / \mathrm{mol}]}\end{array}$ & $\begin{array}{c}M W_{\text {dif }} \\
{[\%]}\end{array}$ \\
\hline & Not assigned & & & & & \\
\hline 78 & $\mathrm{CDCl}_{3}{ }^{\mathrm{b}}$ & $2.1978 \mathrm{E}-09$ & -8.6580 & - & - & - \\
\hline \multirow[t]{2}{*}{152} & $\mathrm{Si}(\mathrm{OMe})_{4}{ }^{\mathrm{b}}$ & $1.5337 \mathrm{E}-09$ & -8.8142 & - & - & - \\
\hline & Compact spheres, $E_{C C}$ & & & & & \\
\hline 70 & Cyclopentane & $2.2338 \mathrm{E}-09$ & -8.6510 & 1.77 & 59 & 18 \\
\hline 72 & THF & $2.0693 \mathrm{E}-09$ & -8.6842 & 1.85 & 70 & 2 \\
\hline 88 & MTBE & $1.8497 \mathrm{E}-09$ & -8.7329 & 1.95 & 90 & -2 \\
\hline 88 & TMS & $1.8894 \mathrm{E}-09$ & -8.7237 & 1.93 & 86 & 3 \\
\hline 114 & TMB & $1.6258 \mathrm{E}-09$ & -8.7889 & 2.08 & 119 & -4 \\
\hline 136 & Adam & $1.5292 \mathrm{E}-09$ & -8.8155 & 2.13 & 136 & 0 \\
\hline 234 & $\mathrm{~N}\left(\mathrm{SiMe}_{3}\right)_{3}$ & $1.2253 \mathrm{E}-09$ & -8.9118 & 2.35 & 221 & 6 \\
\hline \multirow[t]{2}{*}{321} & $\mathrm{Si}\left(\mathrm{SiMe}_{3}\right)_{4}$ & $1.0743 \mathrm{E}-09$ & -8.9689 & 2.47 & 295 & 9 \\
\hline & Dissipated spheres \& ellipsoids, ECC & & & & & \\
\hline 92 & Toluene & $2.0587 \mathrm{E}-09$ & -8.6864 & 1.92 & 83 & 12 \\
\hline 102 & $i \mathrm{Pr}_{2} \mathrm{O}$ & $1.6968 \mathrm{E}-09$ & -8.7704 & 2.06 & 116 & -12 \\
\hline 116 & Indene & $1.7855 \mathrm{E}-09$ & -8.7482 & 2.02 & 106 & 9 \\
\hline 128 & Naphthalene & $1.7976 \mathrm{E}-09$ & -8.7453 & 2.02 & 105 & 22 \\
\hline 146 & 1,3-Indanedione & $1.3693 \mathrm{E}-09$ & -8.8635 & 2.23 & 168 & -13 \\
\hline 155 & 2-PhP & $1.4636 \mathrm{E}-09$ & -8.8346 & 2.18 & 150 & 4 \\
\hline 164 & Tetramethoxypropane & $1.2914 \mathrm{E}-09$ & -8.8889 & 2.27 & 187 & -12 \\
\hline 178 & DPA & $1.3839 \mathrm{E}-09$ & -8.8589 & 2.22 & 165 & 8 \\
\hline 202 & DPS & $1.2028 \mathrm{E}-09$ & -8.9198 & 2.32 & 211 & -4 \\
\hline 204 & $1-\mathrm{PhN}$ & $1.2810 \mathrm{E}-09$ & -8.8925 & 2.28 & 189 & 8 \\
\hline 244 & Triphenylmethane & $1.0922 \mathrm{E}-09$ & -8.9617 & 2.40 & 250 & -2 \\
\hline 304 & Tri(otolyl)phosphine & $9.1243 \mathrm{E}-10$ & -9.0398 & 2.53 & 342 & -11 \\
\hline 595 & Hexaphenyltrisiloxane & $6.8464 \mathrm{E}-10$ & -9.1645 & 2.75 & 566 & 5 \\
\hline \multirow[t]{2}{*}{623} & BINAP & $6.7551 \mathrm{E}-10$ & -9.1704 & 2.76 & 579 & 7 \\
\hline & Expanded discs, $E C C_{E D}$ & & & & & \\
\hline 178 & Anthracene & $1.5341 \mathrm{E}-09$ & -8.8142 & 2.24 & 172 & 3 \\
\hline 179 & Acridine & $1.3732 \mathrm{E}-09$ & -8.8623 & 2.30 & 200 & -10 \\
\hline 192 & 9-MA & $1.4503 \mathrm{E}-09$ & -8.8385 & 2.27 & 186 & 3 \\
\hline 202 & Pyrene & $1.4352 \mathrm{E}-09$ & -8.8431 & 2.28 & 188 & 7 \\
\hline 208 & Anthrachinone & $1.3318 \mathrm{E}-09$ & -8.8756 & 2.32 & 208 & 0 \\
\hline 228 & Triphenylene & $1.2905 \mathrm{E}-09$ & -8.8892 & 2.34 & 217 & 5 \\
\hline 281 & DHBP & $1.0604 \mathrm{E}-09$ & -8.9745 & 2.45 & 282 & 0 \\
\hline \multirow[t]{2}{*}{433} & $\mathrm{TPhN}$ & $7.6453 \mathrm{E}-10$ & -9.1166 & 2.64 & 435 & -1 \\
\hline & & & & & $\varnothing$ & \pm 7 \\
\hline
\end{tabular}

${ }^{a}$ When a compound had more than one resonance in the ${ }^{1} \mathrm{H}$ NMR spectrum, the average diffusion coefficient of all resonances was used.

${ }^{\mathrm{b}} \mathrm{CDCl}_{3}$ and $\mathrm{Si}(\mathrm{OMe})_{4}$ were excluded due to elevated molar van-der-Waals density (see Table 5-24). 
ECCs and Model Compounds for $\mathrm{CD}_{2} \mathrm{Cl}_{2}$
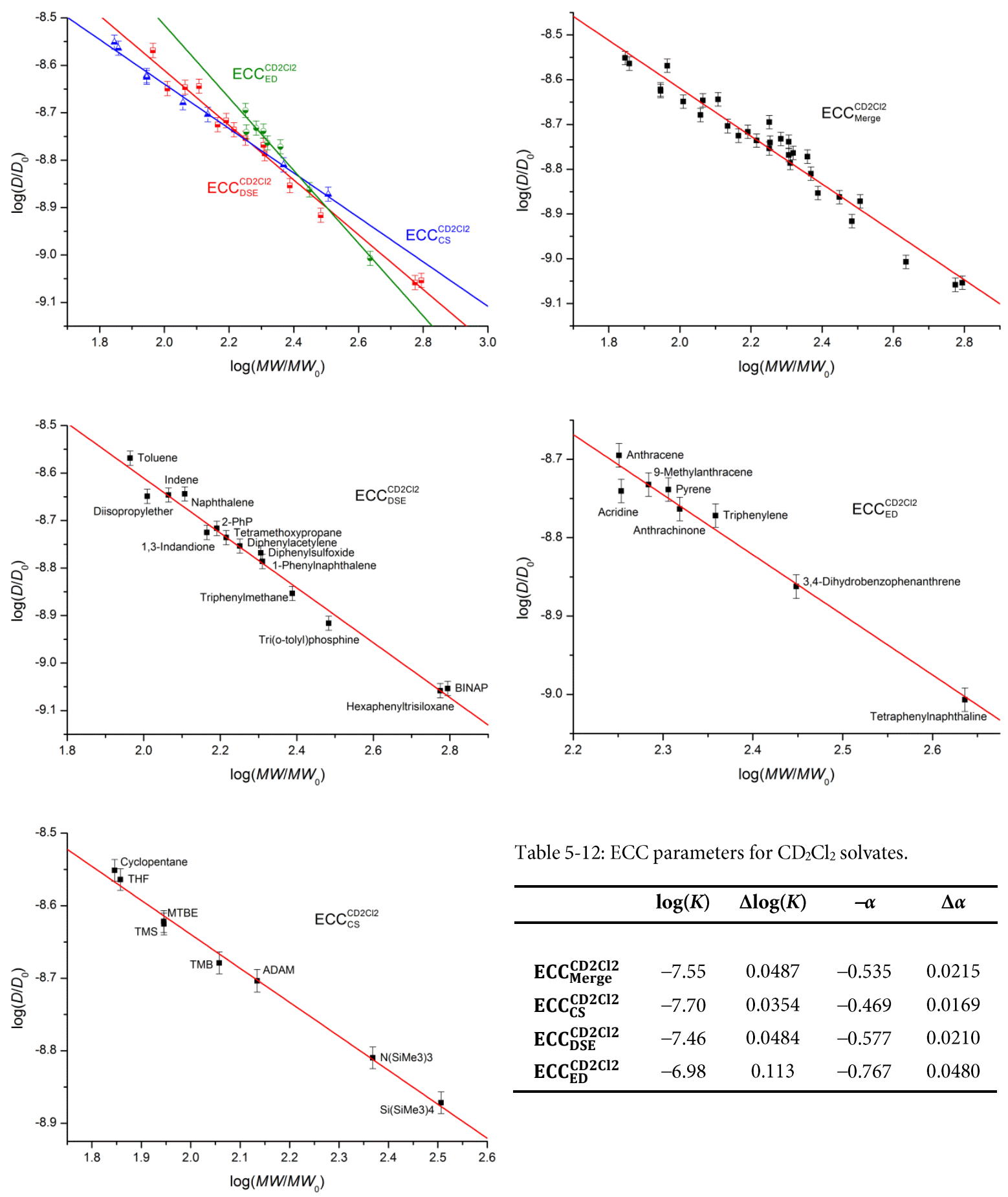

Table 5-12: ECC parameters for $\mathrm{CD}_{2} \mathrm{Cl}_{2}$ solvates.

\begin{tabular}{lcccc}
\hline & $\log (K)$ & $\Delta \log (K)$ & $-\alpha$ & $\Delta \alpha$ \\
\hline & & & & \\
ECC $_{\text {Merge }}^{\text {CD2Cl2 }}$ & -7.55 & 0.0487 & -0.535 & 0.0215 \\
ECC CD2Cl2 $_{\text {CS }}^{\text {CD2 }}$ & -7.70 & 0.0354 & -0.469 & 0.0169 \\
ECC $_{\text {DSE }}^{\text {CD2 }}$ & -7.46 & 0.0484 & -0.577 & 0.0210 \\
ECC $_{\text {ED }}^{\text {CD2Cl2 }}$ & -6.98 & 0.113 & -0.767 & 0.0480 \\
\hline
\end{tabular}

Figure 5-4: Plots of $\log \left(D_{\mathrm{x}, \mathrm{norm}}\right)$ vs. $\log \left(M W_{\text {calc }}\right)$ of all used model compounds in $\mathrm{CD}_{2} \mathrm{Cl}_{2}$ sorted by their molecular shape. There is also a merged ECC of all measured compounds for $M W$ estimation, when the molecular shape of an analyte is unknown. 
Table 5-13: Overview of all used model compounds for $\mathrm{ECC}^{\mathrm{CD} 2 \mathrm{Cl} 2}$ and their normalized diffusion coefficients $\log \left(D_{\mathrm{x}, \mathrm{norm}}\right)$,

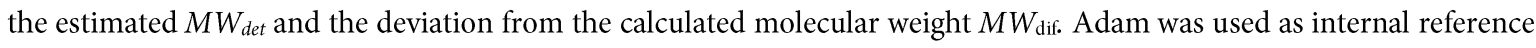
with $\log \left(D_{\text {reffix }}\right)($ Adam $)=-8.7035$. All compounds have been measured as $15 \mathrm{~mm}$ solutions of analyte and Adam in an equimolar ratio. ${ }^{\mathrm{a}}$

\begin{tabular}{|c|c|c|c|c|c|c|}
\hline $\begin{array}{c}M W \\
{[\mathrm{~g} / \mathrm{mol}]}\end{array}$ & & $\begin{array}{l}D_{\mathrm{x}, \text { norm }} \\
{\left[\mathbf{m}^{2} / \mathbf{s}\right]}\end{array}$ & $\begin{array}{c}\log \\
\left(D_{x, \text { norm }}\right) \\
\end{array}$ & $\begin{array}{c}\log \\
\left(M W_{\text {det }}\right)\end{array}$ & $\begin{array}{c}M W_{\text {det }} \\
{[\mathrm{g} / \mathrm{mol}]}\end{array}$ & $\begin{array}{c}M W_{\text {dif }} \\
{[\%]}\end{array}$ \\
\hline & Not assigned & & & & & \\
\hline 78 & $\mathrm{CD}_{2} \mathrm{Cl}_{2}^{\mathrm{b}}$ & $3.1785 \mathrm{E}-09$ & -8.4982 & - & - & - \\
\hline \multirow[t]{2}{*}{152} & $\mathrm{Si}(\mathrm{OMe})_{4}{ }^{\mathrm{b}}$ & $1.9330 \mathrm{E}-09$ & -8.7138 & - & - & - \\
\hline & Compact spheres, $E_{C C}$ & & & & & \\
\hline 70 & Cyclopentane & $2.8292 \mathrm{E}-09$ & -8.5514 & 1.82 & 65 & 7 \\
\hline 72 & THF & $2.7291 \mathrm{E}-09$ & -8.5640 & 1.84 & 70 & 4 \\
\hline 88 & MTBE & $2.3889 \mathrm{E}-09$ & -8.6218 & 1.97 & 92 & -4 \\
\hline 88 & TMS & $2.3700 \mathrm{E}-09$ & -8.6252 & 1.97 & 94 & -6 \\
\hline 114 & TMB & $2.0947 \mathrm{E}-09$ & -8.6789 & 2.09 & 122 & -7 \\
\hline 136 & Adam & $1.9792 \mathrm{E}-09$ & -8.7035 & 2.14 & 138 & -1 \\
\hline 234 & $\mathrm{~N}\left(\mathrm{SiMe}_{3}\right)_{3}$ & $1.5498 \mathrm{E}-09$ & -8.8097 & 2.37 & 232 & 1 \\
\hline \multirow[t]{2}{*}{321} & $\mathrm{Si}\left(\mathrm{SiMe}_{3}\right)_{4}$ & $1.3437 \mathrm{E}-09$ & -8.8717 & 2.50 & 315 & 2 \\
\hline & Dissipated spheres \& ellipsoids, ECC & & & & & \\
\hline 92 & Toluene & $2.6994 \mathrm{E}-09$ & -8.5687 & 1.92 & 83 & 10 \\
\hline 102 & $i \mathrm{Pr}_{2} \mathrm{O}$ & $2.2436 \mathrm{E}-09$ & -8.6490 & 2.06 & 115 & -11 \\
\hline 116 & Indene & $2.2583 \mathrm{E}-09$ & -8.6462 & 2.06 & 114 & 2 \\
\hline 128 & Naphthalene & $2.2702 \mathrm{E}-09$ & -8.6439 & 2.05 & 113 & 14 \\
\hline 146 & 1,3-Indanedione & $1.8832 \mathrm{E}-09$ & -8.7251 & 2.19 & 156 & -6 \\
\hline 155 & 2-PhP & $1.9216 \mathrm{E}-09$ & -8.7163 & 2.18 & 150 & 3 \\
\hline 164 & Tetramethoxypropane & $1.8372 \mathrm{E}-09$ & -8.7358 & 2.21 & 163 & 1 \\
\hline 178 & DPA & $1.7638 \mathrm{E}-09$ & -8.7536 & 2.24 & 175 & 2 \\
\hline 202 & DPS & $1.7061 \mathrm{E}-09$ & -8.7680 & 2.27 & 185 & 9 \\
\hline 204 & $1-\mathrm{PhN}$ & $1.6370 \mathrm{E}-09$ & -8.7860 & 2.30 & 199 & 3 \\
\hline 244 & Triphenylmethane & $1.4018 \mathrm{E}-09$ & -8.8533 & 2.41 & 260 & -6 \\
\hline 304 & Tri(otolyl)phosphine & $1.2127 \mathrm{E}-09$ & -8.9162 & 2.52 & 334 & -9 \\
\hline 595 & Hexaphenyltrisiloxane & $8.7480 \mathrm{E}-10$ & -9.0581 & 2.77 & 588 & 1 \\
\hline \multirow[t]{2}{*}{623} & BINAP & $8.8427 \mathrm{E}-10$ & -9.0534 & 2.76 & 577 & 8 \\
\hline & Expanded discs, $E C C_{E D}$ & & & & & \\
\hline 178 & Anthracene & $2.0189 \mathrm{E}-09$ & -8.6949 & 2.24 & 172 & 3 \\
\hline 179 & Acridine & $1.8180 \mathrm{E}-09$ & -8.7404 & 2.30 & 197 & -9 \\
\hline 192 & 9-MA & $1.8535 \mathrm{E}-09$ & -8.7322 & 2.28 & 192 & 0 \\
\hline 202 & Pyrene & $1.8254 \mathrm{E}-09$ & -8.7386 & 2.29 & 196 & 3 \\
\hline 208 & Anthrachinone & $1.7240 \mathrm{E}-09$ & -8.7635 & 2.33 & 211 & -2 \\
\hline 228 & Triphenylene & $1.6913 \mathrm{E}-09$ & -8.7718 & 2.34 & 217 & 5 \\
\hline 281 & DHBP & $1.3730 \mathrm{E}-09$ & -8.8623 & 2.45 & 285 & -1 \\
\hline \multirow[t]{2}{*}{433} & $\mathrm{TPhN}$ & $9.8418 \mathrm{E}-10$ & -9.0069 & 2.64 & 439 & -2 \\
\hline & & & & & $\varnothing$ & \pm 4 \\
\hline
\end{tabular}

${ }^{a}$ When a compound had more than one resonance in the ${ }^{1} \mathrm{H}$ NMR spectrum, the average diffusion coefficient of all resonances was used.

${ }^{\mathrm{b}} \mathrm{CD}_{2} \mathrm{Cl}_{2}$ and $\mathrm{Si}(\mathrm{OMe})_{4}$ were excluded due to elevated molar van-der-Waals density (see Table 5-24). 
ECCs and Model Compounds for $C_{6} D_{6}$
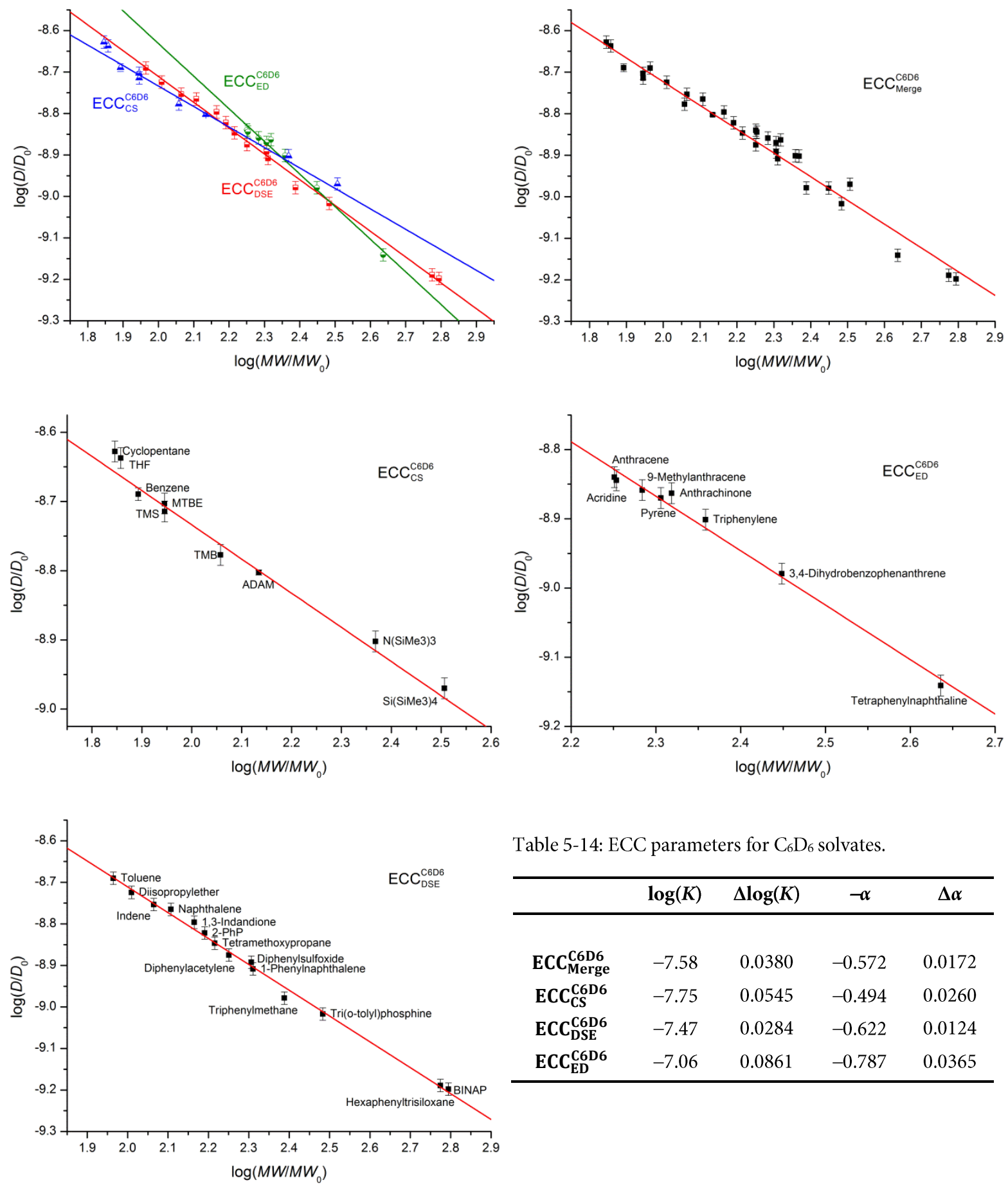

Table 5-14: ECC parameters for $\mathrm{C}_{6} \mathrm{D}_{6}$ solvates.

\begin{tabular}{lllll}
\hline & $\log (K)$ & $\Delta \log (K)$ & $-\alpha$ & $\Delta \alpha$ \\
\hline & & & & \\
ECC $_{\text {Merge }}^{\text {C666 }}$ & -7.58 & 0.0380 & -0.572 & 0.0172 \\
ECC C6 $_{\text {C6 }}^{\text {CD6 }}$ & -7.75 & 0.0545 & -0.494 & 0.0260 \\
ECC $_{\text {DSE }}^{\text {C6D6 }}$ & -7.47 & 0.0284 & -0.622 & 0.0124 \\
ECC $_{\text {ED }}^{\text {C6D6 }}$ & -7.06 & 0.0861 & -0.787 & 0.0365 \\
\hline
\end{tabular}

Figure 5-5: Plots of $\log \left(D_{\mathrm{x}, \mathrm{norm}}\right)$ vs. $\log \left(M W_{\text {calc }}\right)$ of all used model compounds in $\mathrm{C}_{6} \mathrm{D}_{6}$ sorted by their molecular shape. There is also a merged ECC of all measured compounds for $M W$ estimation, when the molecular shape of an analyte is unknown. 
Table 5-15: Overview of all used model compounds for $\mathrm{ECC}^{\mathrm{C} 6 \mathrm{D} 6}$ and their normalized diffusion coefficients $\log \left(D_{\mathrm{x}, \mathrm{norm}}\right)$,

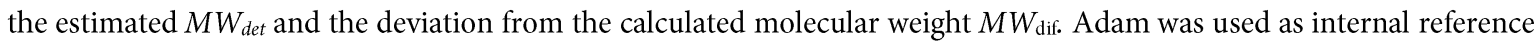
with $\log \left(D_{\text {reffix }}\right)($ Adam $)=-8.8025$. All compounds have been measured as $15 \mathrm{~mm}$ solutions of analyte and Adam in an equimolar ratio. ${ }^{\mathrm{a}}$

\begin{tabular}{|c|c|c|c|c|c|c|}
\hline $\begin{array}{c}M W \\
{[\mathrm{~g} / \mathrm{mol}]}\end{array}$ & & $\begin{array}{l}D_{\mathrm{x}, \text { norm }} \\
{\left[\mathbf{m}^{2} / \mathbf{s}\right]}\end{array}$ & $\begin{array}{c}\log \\
\left(D_{x, \text { norm }}\right) \\
\end{array}$ & $\begin{array}{c}\log \\
\left(M W_{\text {det }}\right)\end{array}$ & $\begin{array}{c}M W_{\text {det }} \\
{[\mathrm{g} / \mathrm{mol}]}\end{array}$ & $\begin{array}{c}M W_{\text {dif }} \\
{[\%]}\end{array}$ \\
\hline & Not assigned & & & & & \\
\hline \multirow[t]{2}{*}{152} & $\mathrm{Si}(\mathrm{OMe})_{4}^{\mathrm{b}}$ & $1.6358 \mathrm{E}-09$ & -8.7863 & - & - & - \\
\hline & Compact spheres, ECC $C S$ & & & & & \\
\hline 70 & Cyclopentane & 2.3723E-09 & -8.6276 & 1.78 & 60 & 17 \\
\hline 72 & THF & $2.3062 \mathrm{E}-09$ & -8.6371 & 1.80 & 62 & 15 \\
\hline 78 & $\mathrm{C}_{6} \mathrm{D}_{6}$ & $2.0446 \mathrm{E}-09$ & -8.6894 & 1.90 & 80 & -2 \\
\hline 88 & MTBE & $1.9816 \mathrm{E}-09$ & -8.7030 & 1.93 & 85 & 4 \\
\hline 88 & TMS & $1.9300 \mathrm{E}-09$ & -8.7144 & 1.95 & 90 & -2 \\
\hline 114 & TMB & $1.6708 \mathrm{E}-09$ & -8.7771 & 2.08 & 120 & -5 \\
\hline 136 & Adam & $1.5757 \mathrm{E}-09$ & -8.8025 & 2.13 & 135 & 1 \\
\hline 234 & $\mathrm{~N}\left(\mathrm{SiMe}_{3}\right)_{3}$ & $1.2529 \mathrm{E}-09$ & -8.9021 & 2.33 & 215 & 9 \\
\hline \multirow[t]{2}{*}{321} & $\mathrm{Si}\left(\mathrm{SiMe}_{3}\right)_{4}$ & $1.0720 \mathrm{E}-09$ & -8.9698 & 2.47 & 295 & 9 \\
\hline & Dissipated spheres \& ellipsoids, ECC & & & & & \\
\hline 92 & Toluene & $2.0415 \mathrm{E}-09$ & -8.6900 & 1.96 & 92 & 1 \\
\hline 102 & $i \mathrm{Pr}_{2} \mathrm{O}$ & $1.8870 \mathrm{E}-09$ & -8.7242 & 2.02 & 104 & -2 \\
\hline 116 & Indene & $1.7647 \mathrm{E}-09$ & -8.7533 & 2.06 & 116 & 0 \\
\hline 128 & Naphthalene & $1.7180 \mathrm{E}-09$ & -8.7650 & 2.08 & 121 & 6 \\
\hline 146 & 1,3-Indanedione & $1.5988 \mathrm{E}-09$ & -8.7962 & 2.13 & 136 & 8 \\
\hline 155 & 2-PhP & $1.5072 \mathrm{E}-09$ & -8.8218 & 2.17 & 149 & 4 \\
\hline 164 & Tetramethoxypropane & $1.4242 \mathrm{E}-09$ & -8.8464 & 2.21 & 163 & 1 \\
\hline 178 & DPA & $1.3338 \mathrm{E}-09$ & -8.8749 & 2.26 & 181 & -2 \\
\hline 202 & DPS & $1.2823 \mathrm{E}-09$ & -8.8920 & 2.29 & 193 & 5 \\
\hline 204 & $1-\mathrm{PhN}$ & $1.2346 \mathrm{E}-09$ & -8.9085 & 2.31 & 205 & -1 \\
\hline 244 & Triphenylmethane & $1.0505 \mathrm{E}-09$ & -8.9786 & 2.43 & 266 & -8 \\
\hline 304 & Tri(otolyl)phosphine & $9.6146 \mathrm{E}-10$ & -9.0171 & 2.49 & 307 & -1 \\
\hline 595 & Hexaphenyltrisiloxane & $6.4699 \mathrm{E}-10$ & -9.1891 & 2.76 & 581 & 2 \\
\hline \multirow[t]{2}{*}{623} & BINAP & $6.3374 \mathrm{E}-10$ & -9.1981 & 2.78 & 600 & 4 \\
\hline & Expanded discs, $E C C_{E D}$ & & & & & \\
\hline 178 & Anthracene & $1.4455 \mathrm{E}-09$ & -8.8400 & 2.26 & 183 & -3 \\
\hline 179 & Acridine & $1.4309 \mathrm{E}-09$ & -8.8444 & 2.27 & 185 & -3 \\
\hline 192 & 9-MA & $1.3831 \mathrm{E}-09$ & -8.8586 & 2.29 & 193 & 0 \\
\hline 202 & Pyrene & $1.3494 \mathrm{E}-09$ & -8.8698 & 2.30 & 199 & 1 \\
\hline 208 & Anthrachinone & $1.3704 \mathrm{E}-09$ & -8.8631 & 2.29 & 196 & 6 \\
\hline 228 & Triphenylene & $1.2555 \mathrm{E}-09$ & -8.9012 & 2.34 & 219 & 4 \\
\hline 281 & DHBP & $1.0487 \mathrm{E}-09$ & -8.9793 & 2.44 & 275 & 2 \\
\hline \multirow[t]{2}{*}{433} & $\mathrm{TPhN}$ & $7.2314 \mathrm{E}-10$ & -9.1408 & 2.64 & 440 & -2 \\
\hline & & & & & $\varnothing$ & \pm 4 \\
\hline
\end{tabular}

${ }^{a}$ When a compound had more than one resonance in the ${ }^{1} \mathrm{H}$ NMR spectrum, the average diffusion coefficient of all resonances was used.

${ }^{\mathrm{b}} \mathrm{Si}(\mathrm{OMe})_{4}$ was excluded due to elevated molar van-der-Waals density (see Table 5-24). 
ECCs and Model Compounds for $\mathrm{CD}_{3} \mathrm{OD}$
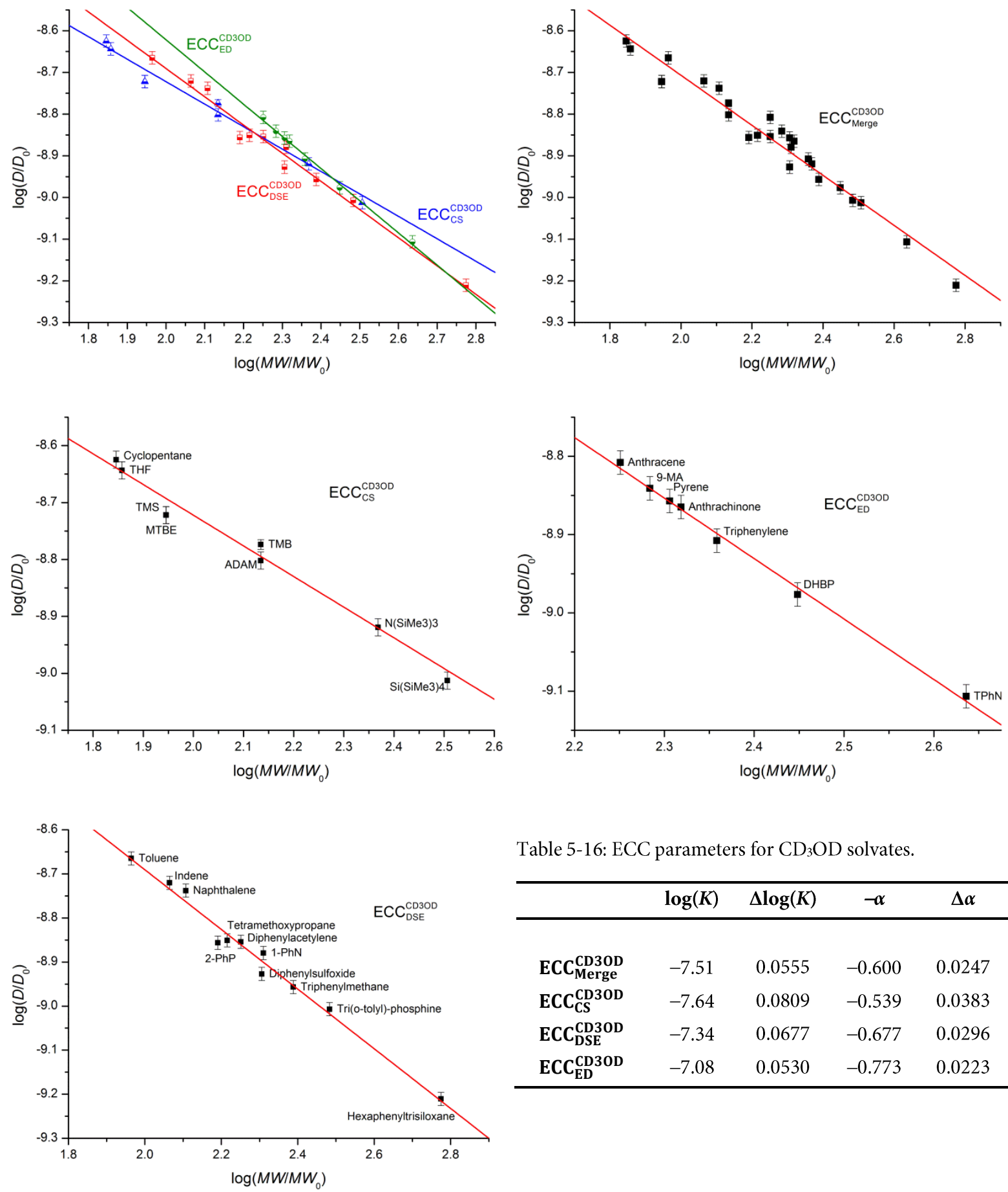

Table 5-16: ECC parameters for $\mathrm{CD}_{3} \mathrm{OD}$ solvates.

\begin{tabular}{lcccc}
\hline & $\log (K)$ & $\Delta \log (K)$ & $-\alpha$ & $\Delta \alpha$ \\
\hline ECC CD30D $_{\text {Merge }}^{\text {CD30 }}$ & -7.51 & 0.0555 & -0.600 & 0.0247 \\
ECC CS CD0D $_{\text {CS }}^{\text {CD30D }}$ & -7.64 & 0.0809 & -0.539 & 0.0383 \\
ECC $_{\text {DSE }}^{\text {CDE }}$ & -7.34 & 0.0677 & -0.677 & 0.0296 \\
ECC CD30D $_{\text {ED }}^{\text {CD }}$ & -7.08 & 0.0530 & -0.773 & 0.0223 \\
\hline
\end{tabular}

Figure 5-6: Plots of $\log \left(D_{\mathrm{x}, \text { norm }}\right)$ vs. $\log \left(M W_{\text {calc }}\right)$ of all used model compounds in $\mathrm{CD}_{3} \mathrm{OD}$ sorted by their molecular shape. There is also a merged ECC of all measured compounds for $M W$ estimation, when the molecular shape of an analyte is unknown. 
Table 5-17: Overview of all used model compounds for ECC ${ }^{\mathrm{CD} 30 \mathrm{D}}$ and their normalized diffusion coefficients $\log \left(D_{\mathrm{x}, \mathrm{norm}}\right)$, the estimated $M W_{\text {det }}$ and the deviation from the calculated molecular weight $M W_{\text {dif. }}$ TMB was used as internal reference with $\log \left(D_{\text {reffix }}\right)($ TMB $)=-8.7737$. All compounds have been measured as $15 \mathrm{mM}$ solutions of analyte and TMB in an equimolar ratio. ${ }^{\mathrm{a}}$

\begin{tabular}{|c|c|c|c|c|c|c|}
\hline $\begin{array}{c}M W \\
{[\mathrm{~g} / \mathrm{mol}]}\end{array}$ & & $\begin{array}{l}D_{\mathrm{x}, \text { norm }} \\
{\left[\mathbf{m}^{2} / \mathbf{s}\right]}\end{array}$ & $\begin{array}{c}\log \\
\left(D_{x, \text { norm }}\right)\end{array}$ & $\begin{array}{c}\log \\
\left(M W_{\text {det }}\right)\end{array}$ & $\begin{array}{c}M W_{\text {det }} \\
{[\mathrm{g} / \mathrm{mol}]}\end{array}$ & $\begin{array}{c}M W_{\text {dif }} \\
{[\%]}\end{array}$ \\
\hline & Not assigned & & & & & \\
\hline 18 & $\mathrm{H}_{2} \mathrm{O}^{\mathrm{b}}$ & $2.0305 \mathrm{E}-10$ & -8.6924 & - & - & - \\
\hline 32 & $\mathrm{CD}_{3} \mathrm{OD}^{\mathrm{b}}$ & $2.0167 \mathrm{E}-10$ & -8.6926 & - & - & - \\
\hline 102 & $i \mathrm{Pr}_{2} \mathrm{O}^{\mathrm{b}}$ & $1.6967 \mathrm{E}-09$ & -8.7704 & - & - & - \\
\hline 146 & 1,3-Indanedione $\mathrm{c}^{\mathrm{c}}$ & $1.4333 \mathrm{E}-09$ & -8.8437 & - & - & - \\
\hline 152 & $\mathrm{Si}(\mathrm{OMe})_{4}{ }^{\mathrm{d}}$ & $1.5757 \mathrm{E}-09$ & -8.8025 & - & - & - \\
\hline \multirow[t]{2}{*}{179} & Acridine $^{c}$ & $1.2788 \mathrm{E}-09$ & -8.8932 & - & - & - \\
\hline & Compact spheres, ECC $C S$ & & & & & \\
\hline 70 & Cyclopentane & $2.3729 \mathrm{E}-09$ & -8.6247 & 1.83 & 67 & 4 \\
\hline 72 & THF & $2.2725 \mathrm{E}-09$ & -8.6435 & 1.86 & 73 & -1 \\
\hline 88 & MTBE & $1.9816 \mathrm{E}-09$ & -8.7030 & 1.97 & 94 & -6 \\
\hline 88 & TMS & $1.8959 \mathrm{E}-09$ & -8.7222 & 2.01 & 102 & -13 \\
\hline 114 & TMB & $1.6840 \mathrm{E}-09$ & -8.7737 & 2.10 & 127 & -10 \\
\hline 136 & Adam & $1.5757 \mathrm{E}-09$ & -8.8025 & 2.16 & 143 & -5 \\
\hline 234 & $\mathrm{~N}\left(\mathrm{SiMe}_{3}\right)_{3}$ & $1.2042 \mathrm{E}-09$ & -8.9193 & 2.37 & 236 & -1 \\
\hline \multirow[t]{2}{*}{321} & $\mathrm{Si}\left(\mathrm{SiMe}_{3}\right)_{4}$ & $9.7101 \mathrm{E}-10$ & -9.0128 & 2.55 & 352 & -9 \\
\hline & Dissipated spheres \& ellipsoids, ECC & & & & & \\
\hline 92 & Toluene & $2.1625 \mathrm{E}-09$ & -8.6650 & 1.96 & 91 & 2 \\
\hline 116 & Indene & $1.9038 \mathrm{E}-09$ & -8.7204 & 2.04 & 109 & 6 \\
\hline 128 & Naphthalene & $1.8273 \mathrm{E}-09$ & -8.7382 & 2.07 & 116 & 10 \\
\hline 155 & 2-PhP & $1.3923 \mathrm{E}-09$ & -8.8563 & 2.24 & 174 & -11 \\
\hline 164 & Tetramethoxypropane & $1.4242 \mathrm{E}-09$ & -8.8464 & 2.23 & 168 & -2 \\
\hline 178 & DPA & $1.3992 \mathrm{E}-09$ & -8.8541 & 2.24 & 172 & 3 \\
\hline 202 & DPS & $1.1827 \mathrm{E}-09$ & -8.9271 & 2.34 & 221 & -8 \\
\hline 204 & $1-\mathrm{PhN}$ & $1.3196 \mathrm{E}-09$ & -8.8796 & 2.27 & 188 & 9 \\
\hline 244 & Triphenylmethane & $1.1046 \mathrm{E}-09$ & -8.9568 & 2.39 & 244 & 0 \\
\hline 304 & Tri(otolyl)phosphine & $9.8373 \mathrm{E}-10$ & -9.0071 & 2.46 & 290 & 5 \\
\hline \multirow[t]{2}{*}{595} & Hexaphenyltrisiloxane & $6.1551 \mathrm{E}-10$ & -9.2108 & 2.76 & 580 & 3 \\
\hline & Expanded discs, ECC $C_{E D}$ & & & & & \\
\hline 178 & Anthracene & $1.5558 \mathrm{E}-09$ & -8.8080 & 2.24 & 172 & 3 \\
\hline 192 & 9-MA & $1.4425 \mathrm{E}-09$ & -8.8409 & 2.28 & 190 & 1 \\
\hline 202 & Pyrene & $1.3897 \mathrm{E}-09$ & -8.8571 & 2.30 & 199 & 2 \\
\hline 208 & Anthrachinone & $1.3649 \mathrm{E}-09$ & -8.8649 & 2.31 & 204 & 2 \\
\hline 228 & Triphenylene & $1.2367 \mathrm{E}-09$ & -8.9078 & 2.36 & 231 & -1 \\
\hline 281 & DHBP & $1.0554 \mathrm{E}-09$ & -8.9766 & 2.45 & 284 & -1 \\
\hline \multirow[t]{2}{*}{433} & $\mathrm{TPhN}$ & $7.8206 \mathrm{E}-10$ & -9.1068 & 2.62 & 419 & 3 \\
\hline & & & & & $\varnothing$ & \pm 4 \\
\hline
\end{tabular}

${ }^{a}$ When a compound had more than one resonance in the ${ }^{1} \mathrm{H}$ NMR spectrum, the average diffusion coefficient of all resonances was used.

${ }^{b} \mathrm{CD}_{3} \mathrm{OD}, i \mathrm{Pr}_{2} \mathrm{O}$ and $\mathrm{H}_{2} \mathrm{O}$ were excluded due to hydrogen-bonding. $\mathrm{H}_{2} \mathrm{O}$ also has an elevated molar van-der-Waals density (see Table 5-24).

${ }^{c}$ 1,3-Indanedione and Acridine were excluded due to unknown aggregational behavior.

${ }^{d} \mathrm{Si}(\mathrm{OMe})_{4}$ was excluded due to elevated molar van-der-Waals density (see Table 5-24). 
ECCs and Model Compounds for $\mathrm{CD}_{3} \mathrm{CN}$
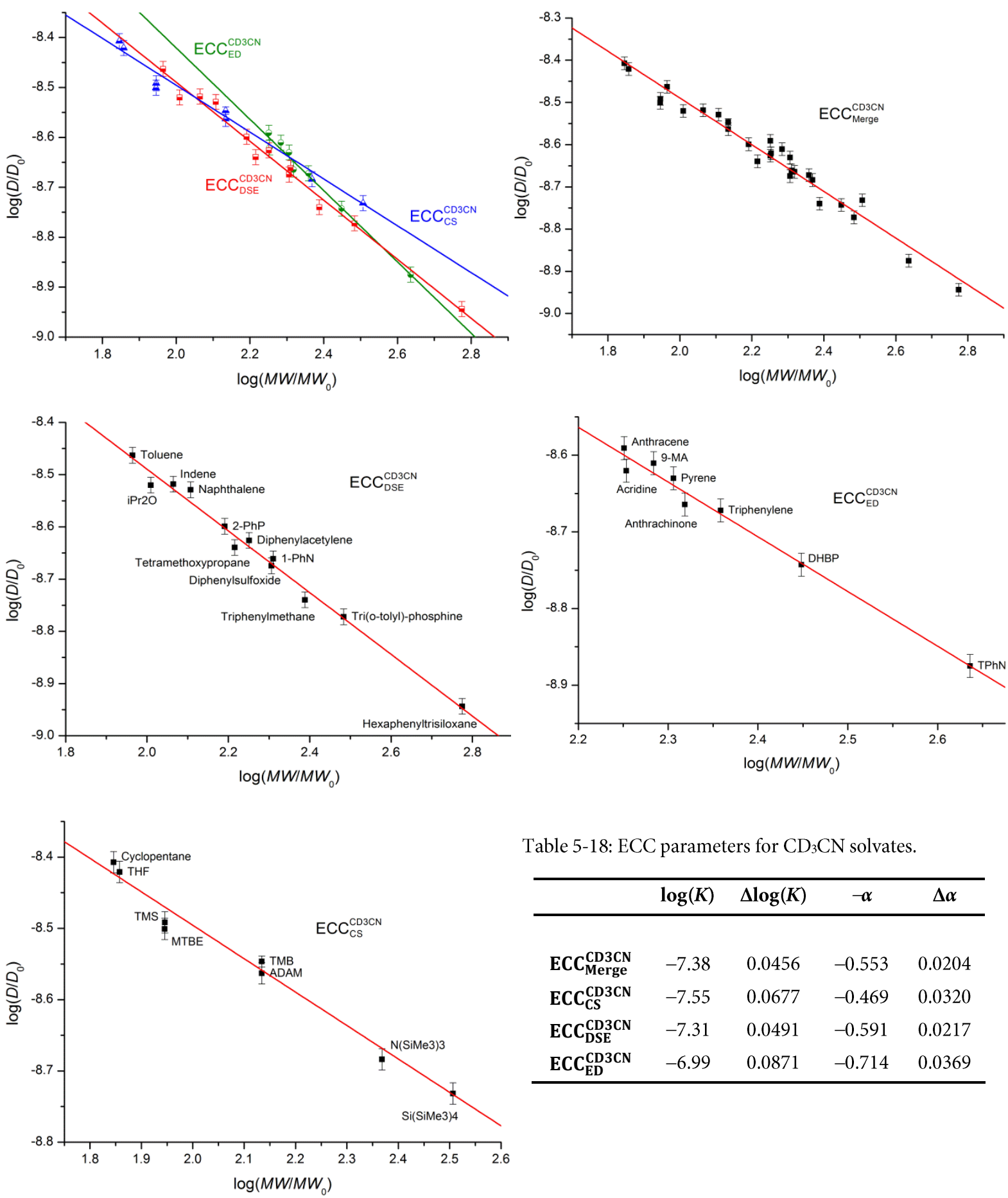

Table 5-18: ECC parameters for $\mathrm{CD}_{3} \mathrm{CN}$ solvates.

\begin{tabular}{lllll}
\hline & $\log (K)$ & $\Delta \log (K)$ & $-\alpha$ & $\Delta \alpha$ \\
\hline & & & & \\
ECC CD3CN $_{\text {Merge }}^{\text {CD3C }}$ & -7.38 & 0.0456 & -0.553 & 0.0204 \\
ECC CS $_{\text {CS }}^{\text {CDN }}$ & -7.55 & 0.0677 & -0.469 & 0.0320 \\
ECC $_{\text {DSE }}^{\text {CD3CN }}$ & -7.31 & 0.0491 & -0.591 & 0.0217 \\
ECC $_{\text {ED }}^{\text {CDCN }}$ & -6.99 & 0.0871 & -0.714 & 0.0369 \\
\hline
\end{tabular}

Figure 5-7: Plots of $\log \left(D_{\mathrm{x}, \mathrm{norm}}\right)$ vs. $\log \left(M W_{\text {calc }}\right)$ of all used model compounds in $\mathrm{CD}_{3} \mathrm{CN}$ sorted by their molecular shape. There is also a merged ECC of all measured compounds for $M W$ estimation, when the molecular shape of an analyte is unknown. 
Table 5-19: Overview of all used model compounds for $\mathrm{ECC}^{\mathrm{CD} 3 \mathrm{CN}}$ and their normalized diffusion coefficients $\log \left(D_{\mathrm{x}, \mathrm{norm}}\right)$, the estimated $M W_{d e t}$ and the deviation from the calculated molecular weight $M W_{\text {dif. }}$ TMB was used as internal reference with $\log \left(D_{\text {reffix }}\right)($ TMB $)=-8.5464$. All compounds have been measured as $15 \mathrm{mM}$ solutions of analyte and TMB in an equimolar ratio. ${ }^{\mathrm{a}}$

\begin{tabular}{|c|c|c|c|c|c|c|}
\hline $\begin{array}{c}M W \\
{[\mathrm{~g} / \mathrm{mol}]}\end{array}$ & & $\begin{array}{l}D_{\mathrm{x}, \text { norm }} \\
{\left[\mathbf{m}^{2} / \mathbf{s}\right]}\end{array}$ & $\begin{array}{c}\log \\
\left(D_{x, \text { norm }}\right) \\
\end{array}$ & $\begin{array}{c}\log \\
\left(M W_{\text {det }}\right)\end{array}$ & $\begin{array}{c}M W_{\text {det }} \\
{[\mathrm{g} / \mathrm{mol}]}\end{array}$ & $\begin{array}{c}M W_{\text {dif }} \\
{[\%]}\end{array}$ \\
\hline & Not assigned & & & & & \\
\hline 18 & $\mathrm{H}_{2} \mathrm{O}^{\mathrm{b}}$ & $5.6678 \mathrm{E}-09$ & -8.2467 & - & - & - \\
\hline 41 & $\mathrm{CD}_{3} \mathrm{CN}^{\mathrm{b}}$ & $4.1844 \mathrm{E}-09$ & -8.3784 & - & - & - \\
\hline \multirow[t]{2}{*}{152} & $\mathrm{Si}(\mathrm{OMe})_{4}^{\mathrm{c}}$ & $2.5507 \mathrm{E}-09$ & -8.5933 & - & - & - \\
\hline & Compact spheres, ECC $C S$ & & & & & \\
\hline 70 & Cyclopentane & $3.9156 \mathrm{E}-09$ & -8.4072 & 1.83 & 67 & 4 \\
\hline 72 & THF & $3.7932 \mathrm{E}-09$ & -8.4210 & 1.86 & 72 & 0 \\
\hline 88 & MTBE & 3.1567E-09 & -8.5007 & 2.03 & 106 & -17 \\
\hline 88 & TMS & $3.2243 \mathrm{E}-09$ & -8.4916 & 2.01 & 102 & -13 \\
\hline 114 & TMB & $2.8600 \mathrm{E}-09$ & -8.5464 & 2.12 & 133 & -14 \\
\hline 136 & Adam & $2.7352 \mathrm{E}-09$ & -8.5630 & 2.16 & 145 & -6 \\
\hline 234 & $\mathrm{~N}\left(\mathrm{SiMe}_{3}\right)_{3}$ & $2.0716 \mathrm{E}-09$ & -8.6837 & 2.42 & 261 & -10 \\
\hline \multirow[t]{2}{*}{321} & $\mathrm{Si}\left(\mathrm{SiMe}_{3}\right)_{4}$ & $1.8548 \mathrm{E}-09$ & -8.7317 & 2.52 & 331 & -3 \\
\hline & Dissipated spheres \& ellipsoids, ECC $C_{D S E}$ & & & & & \\
\hline 92 & Toluene & 3.4439E-09 & -8.4630 & 1.95 & 89 & 3 \\
\hline 102 & $i \operatorname{Pr}_{2} \mathrm{O}$ & $3.0190 \mathrm{E}-09$ & -8.5201 & 2.05 & 112 & -8 \\
\hline 116 & Indene & $3.0319 \mathrm{E}-09$ & -8.5183 & 2.04 & 111 & 5 \\
\hline 128 & Naphthalene & $2.9581 \mathrm{E}-09$ & -8.5290 & 2.06 & 116 & 11 \\
\hline 146 & 1,3-Indanedione & $2.3778 \mathrm{E}-09$ & -8.6238 & 2.22 & 167 & -13 \\
\hline 155 & 2-PhP & $2.5179 \mathrm{E}-09$ & -8.5990 & 2.18 & 152 & 2 \\
\hline 164 & Tetramethoxypropane & $2.2940 \mathrm{E}-09$ & -8.6394 & 2.25 & 178 & -8 \\
\hline 178 & DPA & $2.3667 \mathrm{E}-09$ & -8.6259 & 2.23 & 168 & 6 \\
\hline 202 & DPS & $2.1164 \mathrm{E}-09$ & -8.6744 & 2.31 & 204 & -1 \\
\hline 204 & $1-\mathrm{PhN}$ & $2.1815 \mathrm{E}-09$ & -8.6612 & 2.29 & 193 & 6 \\
\hline 244 & Triphenylmethane & $1.8203 \mathrm{E}-09$ & -8.7398 & 2.42 & 263 & -7 \\
\hline 304 & Tri(otolyl)phosphine & $1.6895 \mathrm{E}-10$ & -8.7722 & 2.47 & 298 & 2 \\
\hline \multirow[t]{2}{*}{595} & Hexaphenyltrisiloxane & $1.1385 \mathrm{E}-09$ & -8.9437 & 2.76 & 581 & 2 \\
\hline & Expanded discs, $E C C_{E D}$ & & & & & \\
\hline 178 & Anthracene & $2.5650 \mathrm{E}-09$ & -8.5909 & 2.24 & 175 & 2 \\
\hline 179 & Acridine & $2.3974 \mathrm{E}-09$ & -8.6203 & 2.28 & 192 & -7 \\
\hline 192 & 9-MA & 2.4517E-09 & -8.6105 & 2.27 & 186 & 3 \\
\hline 202 & Pyrene & $2.3429 \mathrm{E}-09$ & -8.6302 & 2.30 & 198 & 2 \\
\hline 208 & Anthrachinone & $2.1660 \mathrm{E}-09$ & -8.6643 & 2.35 & 221 & -6 \\
\hline 228 & Triphenylene & $2.1282 \mathrm{E}-09$ & -8.6720 & 2.36 & 227 & 1 \\
\hline 281 & DHBP & $1.8080 \mathrm{E}-09$ & -8.7428 & 2.45 & 285 & -1 \\
\hline \multirow[t]{2}{*}{433} & $\mathrm{TPhN}$ & $1.3336 \mathrm{E}-09$ & -8.8750 & 2.64 & 437 & -1 \\
\hline & & & & & $\varnothing$ & \pm 5 \\
\hline
\end{tabular}

${ }^{a}$ When a compound had more than one resonance in the ${ }^{1} \mathrm{H}$ NMR spectrum, the average diffusion coefficient of all resonances was used.

${ }^{b} \mathrm{CD}_{3} \mathrm{CN}$ and $\mathrm{H}_{2} \mathrm{O}$ were excluded due to unknown aggregational behavior. $\mathrm{H}_{2} \mathrm{O}$ also has an elevated molar van-der-Waals density (see Table 5-24).

${ }^{c} \mathrm{Si}(\mathrm{OMe})_{4}$ was excluded due to elevated molar van-der-Waals density (see Table 5-24). 


\section{ECCs and Model Compounds for THF- $d_{8}$ (Rod-Like and Brominated Rod-Like Molecules)}
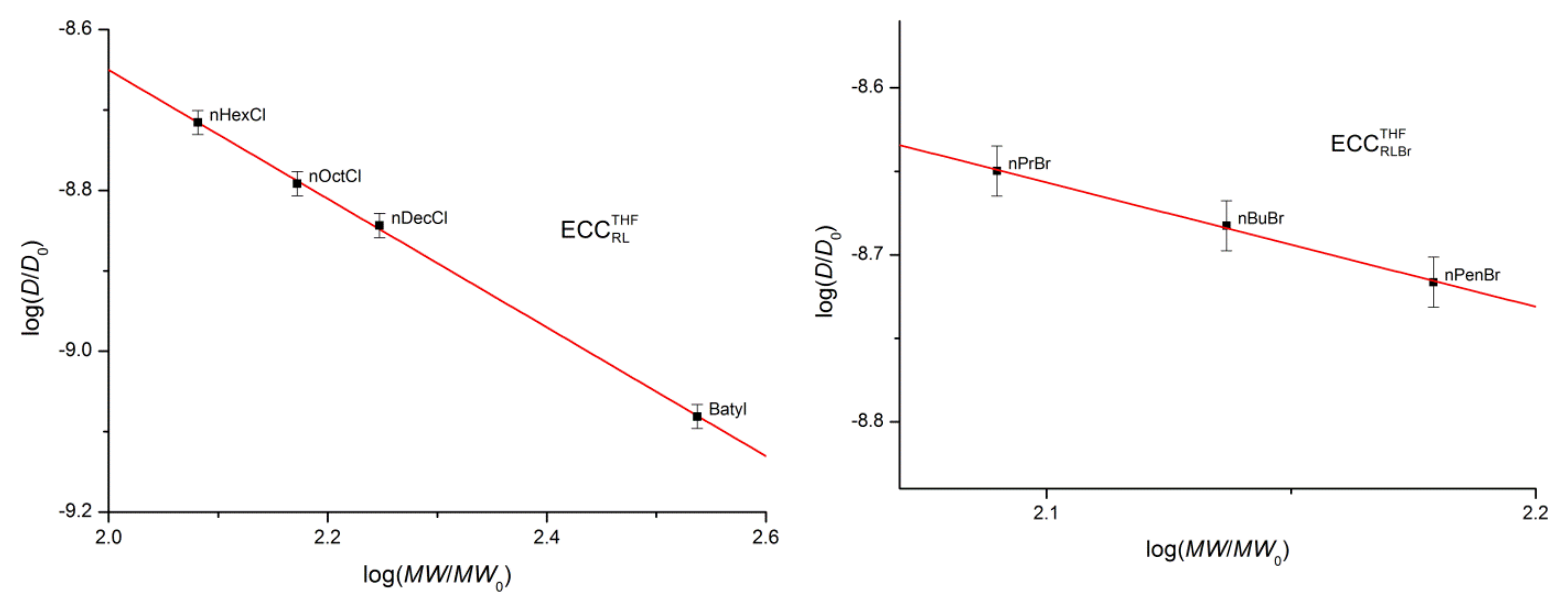

Table 5-20: ECC parameters for THF- $d_{8}$ solvates.

\begin{tabular}{llllc}
\hline & $\log (K)$ & $\Delta \log (K)$ & $-\alpha$ & $\Delta \alpha$ \\
\hline & & & & \\
$\mathbf{E C C}_{\mathbf{R L}}^{\mathbf{T H F}}$ & -7.05 & 0.0277 & -0.800 & 0.0122 \\
$\mathbf{E C C}_{\mathbf{R L B r}}^{\mathbf{T H F}}$ & -7.09 & 0.0625 & -0.745 & 0.0293 \\
\hline
\end{tabular}

Figure 5-8: Plots of $\log \left(D_{\mathrm{x}, \text { norm }}\right)$ vs. $\log \left(M W_{\text {calc }}\right)$ of rod-like $(\mathrm{RL})$ and brominated rod-like (RLBr) model compounds in THF.

Table 5-21: Overview of rod-like model compounds for ECC ${ }^{\mathrm{THF}}$ and their normalized diffusion coefficients $\log \left(D_{\mathrm{x}, \mathrm{norm}}\right)$, the estimated $M W_{d e t}$ and the deviation from the calculated molecular weight $M W_{\text {dif. }}$ TPhN or THF were used as internal references with $\log \left(D_{\text {reffix }}\right)(\mathrm{TPhN})=-9.1054$ or $\log \left(D_{\text {ref,fix }}\right)(\mathrm{THF})=-8.6335$. All compounds have been measured as $25 \mathrm{~mm}$ solutions of analyte and reference in an equimolar ratio. ${ }^{\mathrm{a}}$

\begin{tabular}{|c|c|c|c|c|c|c|}
\hline $\begin{array}{c}M W \\
{[\mathrm{~g} / \mathrm{mol}]}\end{array}$ & & $\begin{array}{l}D_{\mathbf{x}, \text { norm }} \\
{\left[\mathbf{m}^{2} / \mathbf{s}\right]}\end{array}$ & $\begin{array}{c}\log \\
\left(D_{\mathrm{x}, \text { norm }}\right)\end{array}$ & $\begin{array}{c}\log \\
\left(M W_{\text {det }}\right)\end{array}$ & $\begin{array}{c}M W_{\text {det }} \\
{[\mathrm{g} / \mathrm{mol}]}\end{array}$ & $\begin{array}{c}M W_{\text {dif }} \\
{[\%]^{\mathrm{b}}}\end{array}$ \\
\hline & Rod-like molecules, $E C C_{R L}$ & & & & & \\
\hline 121 & $n \mathrm{HexCl}$ & $1.9253 \mathrm{E}-09$ & -8.7155 & 2.08 & 121 & 0 \\
\hline 149 & $n \mathrm{OctCl}$ & $1.6159 \mathrm{E}-09$ & -8.7916 & 2.18 & 150 & -1 \\
\hline 177 & $n \mathrm{DecCl}$ & $1.4338 \mathrm{E}-09$ & -8.8435 & 2.24 & 175 & 1 \\
\hline \multirow[t]{2}{*}{345} & Batyl & 8.2947E-09 & -9.0812 & 2.54 & 346 & 0 \\
\hline & Rod-like brominated molecules, $E_{C C} C_{R L B r}$ & & & & & \\
\hline 123 & $n \mathrm{PrBr}$ & $2.2398 \mathrm{E}-09$ & -8.6498 & 2.09 & 124 & -1 \\
\hline 137 & $n \mathrm{BuBr}$ & $2.0773 \mathrm{E}-09$ & -8.6825 & 2.14 & 137 & 0 \\
\hline \multirow[t]{2}{*}{151} & $n \mathrm{PenBr}$ & $1.9218 \mathrm{E}-10$ & -8.7163 & 2.18 & 152 & -1 \\
\hline & & & & & $\varnothing$ & \pm 1 \\
\hline
\end{tabular}

\footnotetext{
${ }^{a}$ When a compound had more than one resonance in the ${ }^{1} \mathrm{H}$ NMR spectrum, the average diffusion coefficient of all resonances was used.

${ }^{\mathrm{b}}$ Due to the low reference count with very similar references, theoretical errors may be more feasible (see section 2.3.1).
} 


\section{Theoretical Error Analysis of Model Compounds for Various Solvents}

Theoretical errors ( $\left.\Delta M W_{\text {det }}\right)$ have been calculated for model compounds utilizing the formulae discussed in section 2.1.3. ( $\Delta M W_{\text {det,rel }}$ for rod-like molecules are listed in section 2.3.1.1, Table 2-14)

Table 5-22: Calculated relative theoretical errors $\left(\Delta M W_{\text {det,rel }}\right)$ of model compounds and solvents in ECC merge. Due to solubility problems, not all references could be measured in all solvents (marked with ${ }^{\star}$ ).

\begin{tabular}{|c|c|c|c|c|c|c|c|c|c|}
\hline Solvent & DMSO & $\mathrm{C}_{6} \mathrm{D}_{12}$ & $\mathrm{CDCl}_{3}$ & $\mathrm{CD}_{3} \mathrm{OD}$ & $\mathrm{C}_{6} \mathrm{D}_{6}$ & $\mathrm{CD}_{2} \mathrm{Cl}_{2}$ & $\mathrm{CD}_{3} \mathrm{CN}$ & THF & Tol \\
\hline Compound & $\begin{array}{c}\Delta M W \\
{[\%]}\end{array}$ & $\begin{array}{c}\Delta M W \\
{[\%]}\end{array}$ & $\begin{array}{c}\Delta M W \\
{[\%]}\end{array}$ & $\begin{array}{c}\Delta M W \\
{[\%]}\end{array}$ & $\begin{array}{c}\Delta M W \\
{[\%]}\end{array}$ & $\begin{array}{c}\Delta M W \\
{[\%]}\end{array}$ & $\begin{array}{c}\Delta M W \\
{[\%]}\end{array}$ & $\begin{array}{c}\Delta M W \\
{[\%]}\end{array}$ & $\begin{array}{c}\Delta M W \\
{[\%]}\end{array}$ \\
\hline 1,3-Indanedione & * & 11 & 10 & - & 6 & 9 & 8 & 7 & 7 \\
\hline 1-PhN & * & 15 & 10 & 9 & 7 & 9 & 9 & 8 & 7 \\
\hline 2-PhP & 8 & 12 & 10 & 9 & 7 & 9 & 8 & 7 & 7 \\
\hline 9-MA & 8 & 14 & 10 & 9 & 7 & 9 & 8 & 7 & 7 \\
\hline Acridine & 8 & 14 & 10 & 10 & 7 & 9 & 8 & 7 & 7 \\
\hline Adam & 8 & - & - & 9 & - & - & 8 & 7 & - \\
\hline Anthracene & * & 10 & 10 & 9 & 7 & 9 & 8 & 7 & 7 \\
\hline Anthrachinone & 8 & * & 10 & 9 & 7 & 9 & 9 & 8 & 7 \\
\hline BINAP & * & 15 & 13 & * & 9 & 11 & * & 9 & 8 \\
\hline $\mathrm{C}_{6} \mathrm{D}_{6}{ }^{\mathrm{a}}$ & - & - & - & - & 6 & - & - & - & - \\
\hline $\mathrm{CDCl}_{3}^{\mathrm{a}}$ & - & - & 8 & - & - & - & - & - & - \\
\hline $\mathrm{CD}_{2} \mathrm{Cl}_{2}^{\mathrm{a}}$ & - & - & - & - & - & 7 & - & - & - \\
\hline $\mathrm{CD}_{3} \mathrm{CN}^{\mathrm{a}}$ & - & - & - & - & - & - & 7 & - & - \\
\hline $\mathrm{CD}_{3} \mathrm{OD}^{\mathrm{a}}$ & - & - & - & 8 & - & - & - & - & - \\
\hline Cyclohexane- $d_{12^{\mathrm{a}}}$ & - & 12 & - & - & - & - & - & - & - \\
\hline Cyclopentane & 7 & 12 & 8 & 8 & 6 & 8 & 7 & 6 & 6 \\
\hline DHBP & 9 & 14 & 11 & 10 & 7 & 10 & 9 & - & - \\
\hline DPA & 9 & 14 & 10 & 9 & 7 & 9 & 8 & 7 & 7 \\
\hline DPS & 9 & 15 & 11 & 10 & 7 & 9 & 9 & 8 & 7 \\
\hline DMSO- $d_{6}{ }^{\mathrm{a}}$ & 7 & - & - & - & - & - & - & - & - \\
\hline $\mathrm{HexPhTSi}^{\mathrm{b}}$ & 11 & 16 & 13 & 12 & 9 & 11 & 11 & 9 & 8 \\
\hline Indene & 8 & 13 & 9 & 8 & 6 & 8 & 8 & 7 & 6 \\
\hline$i \operatorname{Pr}_{2} \mathrm{O}$ & 7 & 13 & 9 & 9 & 6 & 8 & 8 & 7 & 6 \\
\hline MTBE & 7 & 11 & 9 & 8 & 6 & 8 & 8 & 7 & 6 \\
\hline Naphthalene & 8 & 10 & 9 & 9 & 6 & 8 & 8 & 7 & 6 \\
\hline $\mathbf{N}\left(\mathrm{SiMe}_{3}\right)_{3}$ & 9 & 14 & 11 & 10 & 7 & 10 & 9 & 8 & 7 \\
\hline Pyrene & 9 & 11 & 10 & 9 & 7 & 9 & 8 & 7 & 7 \\
\hline $\mathrm{Si}\left(\mathrm{SiMe}_{3}\right)_{4}$ & 9 & 16 & 11 & 10 & 7 & 10 & 9 & 8 & 7 \\
\hline $\mathrm{TMePr}^{\mathrm{b}}$ & 8 & 14 & 10 & 9 & 7 & 9 & 8 & 7 & 7 \\
\hline TPhN & 10 & 17 & 12 & 11 & 8 & 11 & 10 & 9 & 8 \\
\hline THF & 7 & 11 & 9 & 8 & 6 & 8 & 7 & - & 6 \\
\hline TMB & - & 14 & 10 & - & 6 & 9 & - & - & 6 \\
\hline TMS & 7 & 12 & 9 & 8 & 6 & 8 & 8 & 7 & 6 \\
\hline Toluene & 7 & 11 & 9 & 8 & 6 & 8 & 7 & 6 & - \\
\hline TTolPh $^{\mathbf{b}}$ & 9 & 16 & 12 & 10 & 8 & 10 & 9 & 8 & 8 \\
\hline Triphenylene & 9 & 14 & 10 & 10 & 7 & 9 & 9 & 8 & 7 \\
\hline $\mathrm{TPhMe}^{\mathrm{b}}$ & 9 & 16 & 11 & 10 & 7 & 10 & 9 & - & - \\
\hline Water & - & - & - & 8 & - & - & 6 & - & - \\
\hline$\varnothing$ & 8 & 13 & 10 & 9 & 7 & 9 & 8 & 7 & 7 \\
\hline
\end{tabular}

Table 5-23: Calculated relative theoretical errors $\left(\Delta M W_{\text {det,rel }}\right)$ of model compounds and solvents in their corresponding shape-optimized ECCs (ECCDSE, ECCCS, ECCED). References not fitting to any ECC and internal references are left out.

\footnotetext{
a Residual solvent signal.

${ }^{\mathrm{b}} \mathrm{HexPhTSi}=$ Hexamethyltrisiloxane; $\mathrm{TMePr}=$ Tetramethoxypropane; $\mathrm{TTolPh}=\mathrm{Tri}($ otolyl $)$ phosphine; $\mathrm{TPhMe}=$ Triphenylmethane.
} 
Due to solubility problems, not every reference could be measured in all solvents (marked with *). For average errors of the individual shape optimized ECCs see section 2.1.3.

\begin{tabular}{|c|c|c|c|c|c|c|c|c|c|}
\hline Solvent & DMSO & $\mathrm{C}_{6} \mathrm{D}_{12}$ & $\mathrm{CDCl}_{3}$ & $\mathrm{CD}_{3} \mathrm{OD}$ & $\mathrm{C}_{6} \mathrm{D}_{6}$ & $\mathrm{CD}_{2} \mathrm{Cl}_{2}$ & $\mathrm{CD}_{3} \mathrm{CN}$ & THF & Tol \\
\hline Compound & $\begin{array}{c}\Delta M W \\
{[\%]}\end{array}$ & $\begin{array}{c}\Delta M W \\
{[\%]}\end{array}$ & $\begin{array}{c}\Delta M W \\
{[\%]}\end{array}$ & $\begin{array}{c}\Delta M W \\
{[\%]}\end{array}$ & $\begin{array}{c}\Delta M W \\
{[\%]}\end{array}$ & $\begin{array}{c}\Delta M W \\
{[\%]}\end{array}$ & $\begin{array}{c}\Delta M W \\
{[\%]}\end{array}$ & $\begin{array}{c}\Delta M W \\
{[\%]}\end{array}$ & $\begin{array}{c}\Delta M W \\
{[\%]}\end{array}$ \\
\hline 1,3-Indanedione & $*$ & 9 & 12 & - & 4 & 8 & 9 & 5 & 4 \\
\hline 1-PhN & * & 9 & 12 & 10 & 5 & 8 & 9 & 5 & 4 \\
\hline 2-PhP & 7 & 8 & 12 & 10 & 4 & 8 & 9 & 5 & 4 \\
\hline 9-MA & 8 & 10 & 17 & 7 & 11 & 14 & 12 & 6 & 9 \\
\hline Acridine & 8 & 10 & 17 & 7 & 11 & 15 & 12 & 6 & 9 \\
\hline Adam & 19 & - & - & 15 & - & - & 15 & 8 & - \\
\hline Anthracene & * & 10 & 17 & 7 & 11 & 14 & 12 & 6 & 9 \\
\hline Anthrachinone & 8 & * & 18 & 7 & 11 & 15 & 12 & 6 & 9 \\
\hline BINAP & * & 11 & 15 & * & 6 & 10 & * & 6 & 5 \\
\hline $\mathrm{C}_{6} \mathrm{D}_{6}{ }^{\mathrm{a}}$ & - & - & - & - & 10 & - & - & - & - \\
\hline Cyclohexane- $d_{12}{ }^{\mathrm{a}}$ & - & 15 & - & - & - & - & - & - & - \\
\hline Cyclopentane & 16 & 14 & 9 & 13 & 9 & 7 & 13 & 7 & 8 \\
\hline DHBP & 8 & 11 & 19 & 7 & 11 & 16 & 13 & - & - \\
\hline DPA & 7 & 9 & 12 & 10 & 5 & 8 & 9 & 5 & 4 \\
\hline DPS & 7 & 9 & 12 & 10 & 5 & 8 & 9 & 5 & 4 \\
\hline $\mathrm{HexPhTSi}^{\mathrm{b}}$ & 8 & 11 & 15 & 12 & 6 & 10 & 11 & 6 & 5 \\
\hline Indene & 6 & 8 & 11 & 9 & 4 & 8 & 8 & 4 & 4 \\
\hline$i \mathrm{Pr}_{2} \mathrm{O}$ & 6 & 8 & 11 & 9 & 4 & 8 & 8 & 4 & 4 \\
\hline MTBE & 17 & 14 & 10 & 14 & 10 & 7 & 14 & 7 & 9 \\
\hline Naphthalene & 6 & 8 & 11 & 9 & 4 & 8 & 8 & 4 & 4 \\
\hline $\mathrm{N}\left(\mathrm{SiMe}_{3}\right)_{3}$ & 21 & 17 & 12 & 17 & 12 & 9 & 17 & 9 & 11 \\
\hline Pyrene & 8 & 10 & 17 & 6 & 11 & 15 & 12 & 6 & 9 \\
\hline $\mathrm{Si}\left(\mathrm{SiMe}_{3}\right)_{4}$ & 22 & 18 & 13 & 18 & 13 & 9 & 17 & 10 & 11 \\
\hline TMePr ${ }^{b}$ & 7 & 9 & 12 & 10 & 4 & 8 & 9 & 5 & 4 \\
\hline TPhN & 9 & 12 & 20 & 8 & 12 & 17 & 14 & 7 & 10 \\
\hline THF & 16 & 13 & 9 & 13 & 10 & 7 & 13 & - & 8 \\
\hline TMB & - & 16 & 11 & - & 11 & 8 & - & - & 9 \\
\hline TMS & 17 & 15 & 10 & 14 & 10 & 7 & 14 & 7 & 9 \\
\hline Toluene & 6 & 8 & 10 & 9 & 4 & 7 & 8 & 4 & - \\
\hline TTolPh $^{\mathbf{b}}$ & 8 & 10 & 14 & 11 & 5 & 9 & 10 & 5 & 4 \\
\hline Triphenylene & 8 & 11 & 18 & 7 & 11 & 15 & 12 & 6 & 9 \\
\hline $\mathrm{TPhMe}^{\mathrm{b}}$ & 7 & 10 & 13 & 11 & 5 & 9 & 9 & - & - \\
\hline
\end{tabular}

${ }^{a}$ Residual solvent signal.

${ }^{\mathrm{b}}$ HexPhTSi = Hexamethyltrisiloxane; TMePr = Tetramethoxypropane; TTolPh = Tri (otolyl)phosphine; TPhMe $=$ Triphenylmethane. 


\section{Molar Van-Der-Waals Densities $\left(M D_{W}\right)$ of Model Compounds}

Molar van-der-Waals densities $\left(M D_{\mathrm{W}}\right)$ for all model compounds and proposed solution state structures have been calculated as discussed in section 2.1.4. $M D_{\mathrm{w}}$ for proposed solution state structures are given in the following sections after the corresponding ECC- $M W$ estimations. The ECC- $M W$ estimation works best with molecules that have a molar van-der-Waals density between $M D_{\mathrm{W}}=4.30 \cdot 10^{29} \mathrm{~g} /\left(\mathrm{mol} \cdot \mathrm{m}^{3}\right)$ and $M D_{\mathrm{W}}=5.92 \cdot 10^{29} \mathrm{~g} /\left(\mathrm{mol} \cdot \mathrm{m}^{3}\right)$, if no correction factor is applied. If $M D_{\mathrm{W}}$ is bigger than $5.92 \cdot 10^{29} \mathrm{~g} /\left(\mathrm{mol} \cdot \mathrm{m}^{3}\right)$, like e.g. for $\mathrm{CHCl}_{3} / \mathrm{CH}_{2} \mathrm{Cl}_{2}$ (marked red) then the predicted $M W$ would be underestimated. ${ }^{[109 a]}$

Table 5-24: $M D_{\mathrm{W}}$ for model compounds used for ECCs; except for rod-like molecules.

\begin{tabular}{|c|c|c|c|c|c|c|c|c|c|c|c|}
\hline Model Compound & $\begin{array}{c}M W \\
{[\mathrm{~g} / \mathrm{mol}]}\end{array}$ & $\mathrm{C}$ & $\mathbf{H}$ & $\mathbf{O}$ & $\mathbf{N}$ & $\mathbf{S}$ & $\mathrm{Cl}$ & $\mathbf{P}$ & Si & $\begin{array}{c}M D_{\mathrm{W}} \\
{\left[\mathrm{g} /\left(\mathbf{m o l} \cdot \mathbf{m}^{3}\right)\right]}\end{array}$ & $\begin{array}{l}\Sigma V_{W} \\
{\left[\mathrm{~m}^{3}\right]}\end{array}$ \\
\hline 1-PhN & 204 & 16 & 12 & 0 & 0 & 0 & 0 & 0 & 0 & $5.16 \mathrm{E}+29$ & $3.95 \mathrm{E}-28$ \\
\hline 1,3-Indanedione & 146 & 9 & 6 & 2 & 0 & 0 & 0 & 0 & 0 & $5.89 \mathrm{E}+29$ & $2.48 \mathrm{E}-28$ \\
\hline 2-PhP & 155 & 11 & 9 & 0 & 1 & 0 & 0 & 0 & 0 & $5.31 \mathrm{E}+29$ & $2.93 \mathrm{E}-28$ \\
\hline 2,2'-Methylenbisbenzothiazol & 282 & 15 & 10 & 0 & 2 & 2 & 0 & 0 & 0 & $6.35 \mathrm{E}+29$ & $4.44 \mathrm{E}-28$ \\
\hline 9-MA & 192 & 15 & 12 & 0 & 0 & 0 & 0 & 0 & 0 & $5.12 \mathrm{E}+29$ & $3.75 \mathrm{E}-28$ \\
\hline Acridine & 179 & 13 & 9 & 0 & 1 & 0 & 0 & 0 & 0 & $5.38 \mathrm{E}+29$ & $3.33 \mathrm{E}-28$ \\
\hline Adam & 136 & 10 & 16 & 0 & 0 & 0 & 0 & 0 & 0 & $4.62 \mathrm{E}+29$ & $2.94 \mathrm{E}-28$ \\
\hline Anthracene & 178 & 14 & 10 & 0 & 0 & 0 & 0 & 0 & 0 & $5.18 \mathrm{E}+29$ & $3.44 \mathrm{E}-28$ \\
\hline Anthrachinone & 208 & 14 & 8 & 2 & 0 & 0 & 0 & 0 & 0 & $5.75 \mathrm{E}+29$ & $3.62 \mathrm{E}-28$ \\
\hline BINAP & 623 & 44 & 32 & 0 & 0 & 0 & 0 & 2 & 0 & $5.50 \mathrm{E}+29$ & $1.13 \mathrm{E}-27$ \\
\hline $\mathrm{C}_{6} \mathrm{H}_{6}$ & 78 & 6 & 6 & 0 & 0 & 0 & 0 & 0 & 0 & $4.98 \mathrm{E}+29$ & $1.57 \mathrm{E}-28$ \\
\hline $\mathrm{CHCl}_{3}$ & 119 & 1 & 1 & 0 & 0 & 0 & 3 & 0 & 0 & $12.8 \mathrm{E}+29$ & $9.30 \mathrm{E}-29$ \\
\hline $\mathrm{CH}_{2} \mathrm{Cl}_{2}$ & 85 & 1 & 2 & 0 & 0 & 0 & 2 & 0 & 0 & $11.1 \mathrm{E}+29$ & $7.66 \mathrm{E}-29$ \\
\hline $\mathrm{CH}_{3} \mathrm{CN}$ & 41 & 2 & 3 & 0 & 1 & 0 & 0 & 0 & 0 & $5.59 \mathrm{E}+29$ & $7.33 \mathrm{E}-29$ \\
\hline $\mathrm{CH}_{3} \mathrm{OH}$ & 32 & 1 & 4 & 1 & 0 & 0 & 0 & 0 & 0 & $5.56 \mathrm{E}+29$ & $5.76 \mathrm{E}-29$ \\
\hline Cyclohexane & 84 & 6 & 12 & 0 & 0 & 0 & 0 & 0 & 0 & $4.42 \mathrm{E}+29$ & $1.90 \mathrm{E}-28$ \\
\hline Cyclopentane & 70 & 5 & 10 & 0 & 0 & 0 & 0 & 0 & 0 & $4.42 \mathrm{E}+29$ & $1.58 \mathrm{E}-28$ \\
\hline DHBP & 280 & 22 & 16 & 0 & 0 & 0 & 0 & 0 & 0 & $5.17 \mathrm{E}+29$ & $5.42 \mathrm{E}-28$ \\
\hline DPA & 178 & 14 & 10 & 0 & 0 & 0 & 0 & 0 & 0 & $5.18 \mathrm{E}+29$ & $3.44 \mathrm{E}-28$ \\
\hline DPS & 202 & 12 & 10 & 1 & 0 & 1 & 0 & 0 & 0 & $5.92 \mathrm{E}+29$ & $3.41 \mathrm{E}-28$ \\
\hline DMSO & 78 & 2 & 6 & 1 & 0 & 1 & 0 & 0 & 0 & $6.87 \mathrm{E}+29$ & $1.14 \mathrm{E}-28$ \\
\hline Hexaphenyltrisiloxane & 595 & 36 & 30 & 3 & 0 & 0 & 0 & 0 & 3 & $5.57 \mathrm{E}+29$ & $1.07 \mathrm{E}-27$ \\
\hline Indene & 116 & 9 & 8 & 0 & 0 & 0 & 0 & 0 & 0 & $5.05 \mathrm{E}+29$ & $2.30 \mathrm{E}-28$ \\
\hline$i \operatorname{Pr}_{2} \mathrm{O}$ & 102 & 6 & 14 & 1 & 0 & 0 & 0 & 0 & 0 & $4.73 \mathrm{E}+29$ & $2.16 \mathrm{E}-28$ \\
\hline MTBE & 88 & 5 & 12 & 1 & 0 & 0 & 0 & 0 & 0 & $4.78 \mathrm{E}+29$ & $1.84 \mathrm{E}-28$ \\
\hline Naphthalene & 128 & 10 & 8 & 0 & 0 & 0 & 0 & 0 & 0 & $5.12 \mathrm{E}+29$ & $2.50 \mathrm{E}-28$ \\
\hline $\mathbf{N}\left(\mathrm{SiMe}_{3}\right)_{3}$ & 234 & 9 & 27 & 0 & 1 & 0 & 0 & 0 & 3 & $4.99 \mathrm{E}+29$ & $4.69 \mathrm{E}-28$ \\
\hline Pyrene & 202 & 16 & 10 & 0 & 0 & 0 & 0 & 0 & 0 & $5.25 \mathrm{E}+29$ & $3.85 \mathrm{E}-28$ \\
\hline $\mathrm{Si}(\mathrm{OMe})_{4}$ & 152 & 4 & 12 & 4 & 0 & 0 & 0 & 0 & 1 & $6.17 \mathrm{E}+29$ & $2.46 \mathrm{E}-28$ \\
\hline $\mathrm{Si}\left(\mathrm{SiMe}_{3}\right)_{4}$ & 321 & 12 & 36 & 0 & 0 & 0 & 0 & 0 & 5 & $5.00 \mathrm{E}+29$ & $6.42 \mathrm{E}-28$ \\
\hline Tetramethoxypropane & 164 & 7 & 16 & 4 & 0 & 0 & 0 & 0 & 0 & $5.62 \mathrm{E}+29$ & $2.92 \mathrm{E}-28$ \\
\hline $\mathrm{TPhN}$ & 433 & 34 & 24 & 0 & 0 & 0 & 0 & 0 & 0 & $5.19 \mathrm{E}+29$ & $8.34 \mathrm{E}-28$ \\
\hline THF & 72 & 4 & 8 & 1 & 0 & 0 & 0 & 0 & 0 & $5.09 \mathrm{E}+29$ & $1.41 \mathrm{E}-28$ \\
\hline TMB & 114 & 8 & 18 & 0 & 0 & 0 & 0 & 0 & 0 & $4.31 \mathrm{E}+29$ & $2.65 \mathrm{E}-28$ \\
\hline TMS & 88 & 4 & 12 & 0 & 0 & 0 & 0 & 0 & 1 & $4.69 \mathrm{E}+29$ & $1.88 \mathrm{E}-28$ \\
\hline Toluene & 92 & 7 & 8 & 0 & 0 & 0 & 0 & 0 & 0 & $4.88 \mathrm{E}+29$ & $1.89 \mathrm{E}-28$ \\
\hline Tri(otolyl)phosphine & 304 & 21 & 21 & 0 & 0 & 0 & 0 & 1 & 0 & $5.31 \mathrm{E}+29$ & $5.73 \mathrm{E}-28$ \\
\hline Triphenylene & 228 & 18 & 12 & 0 & 0 & 0 & 0 & 0 & 0 & $5.22 \mathrm{E}+29$ & $4.37 \mathrm{E}-28$ \\
\hline Triphenylmethane & 244 & 19 & 16 & 0 & 0 & 0 & 0 & 0 & 0 & $5.09 \mathrm{E}+29$ & $4.80 \mathrm{E}-28$ \\
\hline
\end{tabular}


Table 5-25: $M D_{\mathrm{W}}$ and $\log \left(D_{\mathrm{x}, \mathrm{norm}}\right)$ for all reference compounds used by Wang and Pedersen et al. for their ${ }^{1} \mathrm{H}-\mathrm{DOSY}$ ECC in $\mathrm{CD}_{2} \mathrm{Cl}_{2}{ }^{[113]}$

\begin{tabular}{lcccccccccccc}
\hline Model Compound & $\begin{array}{c}\boldsymbol{M} W \\
{[\mathbf{g} / \mathbf{m o l}]}\end{array}$ & $\mathbf{C}$ & $\mathbf{H}$ & $\mathbf{O}$ & $\mathbf{F}$ & $\mathbf{B}$ & $\mathbf{N}$ & $\mathbf{C l}$ & $\mathbf{S i}$ & $\mathbf{S}$ & $\begin{array}{c}\boldsymbol{M D}_{\mathrm{W}} \\
{\left[\mathbf{g} /\left(\mathbf{m o l} \cdot \mathbf{m}^{3}\right)\right]}\end{array}$ & $\log \left(\boldsymbol{D}_{\mathbf{x}, \text { norm }}\right)$ \\
\hline $\mathbf{E t}_{2} \mathbf{O}$ & 76 & 4 & 12 & 1 & 0 & 0 & 0 & 0 & 0 & 0 & $4.64 \mathrm{E}+29$ & -8.5724 \\
$\mathbf{B F}_{\mathbf{3}} \cdot \mathbf{E t}_{\mathbf{2}} \mathbf{O}$ & 144 & 4 & 12 & 1 & 3 & 1 & 0 & 0 & 0 & 0 & $6.16 \mathrm{E}+29$ & -8.6364 \\
$\mathbf{A}^{*}$ & 390 & 16 & 22 & 11 & 0 & 0 & 0 & 0 & 0 & 0 & $6.36 \mathrm{E}+29$ & -8.7342 \\
$\mathbf{B}^{*}$ & 541 & 21 & 24 & 9 & 0 & 0 & 1 & 3 & 0 & 0 & $6.92 \mathrm{E}+29$ & -8.7578 \\
$\mathbf{C}^{*}$ & 610 & 28 & 8 & 0 & 14 & 0 & 0 & 0 & 0 & 0 & $7.56 \mathrm{E}+29$ & -8.7759 \\
$\mathbf{D}^{*}$ & 1226 & 76 & 88 & 5 & 0 & 0 & 0 & 0 & 4 & 1 & $5.31 \mathrm{E}+29$ & -8.8429 \\
\hline
\end{tabular}

*Abbreviations according to Scheme 2-1. 


\section{ECC-MW Estimations of CpM Derivatives ( $\mathrm{M}=\mathrm{Li}, \mathrm{Na}, \mathrm{K}, \mathrm{Rb}, \mathrm{Cs}$ )}

\section{ECC-MW Estimation of CpLi in THF and DMSO}

Table 5-26: ${ }^{1} \mathrm{H}$ - and ${ }^{7} \mathrm{Li}-\mathrm{DOSY}-\mathrm{ECC}-M W$ estimation of CpLi in THF- $d_{8}(15 \mathrm{mM})$ at different temperatures. TPhN or TMB were used as internal references with $\log \left(D_{\text {ref,fix }}\right)(\mathrm{TPhN})=-9.1054$ or $\log \left(D_{\text {ref,fix }}\right)(\mathrm{TMB})=-8.7749$. The accuracy of the $\mathrm{ECC}^{\mathrm{THF}}(\mathrm{DSE})$ is in the range of $M W_{\mathrm{dif}}= \pm 8 \%$. Hypothetical aggregates are $\left[\mathrm{CpLi}(\mathrm{THF})_{\mathrm{x}}\right]$ and $\left[(\mathrm{CpLi})_{2}(\mathrm{THF})_{\mathrm{x}}\right]$ with $\mathrm{x}=1$ 4. A trend is visible towards higher $M W$ s upon cooling, indicating the formation of a [C $\left.\mathrm{Cp}_{2} \mathrm{Li}\right]^{-}$species proposed by Paquette et al. ${ }^{[66]}$. Still, note that $[\mathrm{CpLi}(\mathrm{THF})]$ has a similar $M W$ as $\left[\mathrm{Cp}_{2} \mathrm{Li}\right]^{-},\left[\mathrm{CpLi}(\mathrm{THF})_{2}\right]$ as $\left[\mathrm{Cp}_{2} \mathrm{Li}(\mathrm{THF})\right]^{-}$and so on. For ECC ${ }^{\mathrm{THF}}$ parameters and changes compared to literature ${ }^{[109 \mathrm{a}]}$ see section 2.1.1.

\begin{tabular}{|c|c|c|c|c|}
\hline${ }^{1} \mathrm{H}-\mathrm{DOSY}$ & & & & $50^{\circ} \mathrm{C}$ \\
\hline & & Aggregate & $M W_{\text {calc }}[\mathrm{g} / \mathrm{mol}]$ & $M W_{\text {dif }}[\%]$ \\
\hline$D_{\mathrm{x}}\left[\mathrm{m}^{2} / \mathrm{s}\right]$ & $2.175 \mathrm{E}-09$ & [CpLi(THF)] & 144 & -30 \\
\hline $\log \left(D_{\mathbf{x}}\right)$ & -8.6625 & {$\left[\mathrm{CpLi}(\mathrm{THF})_{2}\right]$} & 216 & 5 \\
\hline $\log \left(D_{\mathrm{x}, \text { norm }}\right)$ & -8.8855 & {$\left[\mathrm{CpLi}(\mathrm{THF})_{3}\right]$} & 288 & 41 \\
\hline$D_{\text {ref }}(\mathrm{TMB})\left[\mathrm{m}^{2} / \mathrm{s}\right]$ & $2.806 \mathrm{E}-09$ & {$\left[\mathrm{CpLi}(\mathrm{THF})_{4}\right]$} & 360 & 76 \\
\hline $\log \left(D_{\text {ref }}\right)($ TMB $)$ & -8.5519 & {$\left[(\mathrm{CpLi})_{2}(\mathrm{THF})\right]$} & 216 & 5 \\
\hline$M W_{\text {det }}[\mathrm{g} / \mathrm{mol}]$ & 205 & {$\left[(\mathrm{CpLi})_{2}(\mathrm{THF})_{2}\right]$} & 288 & 41 \\
\hline \multirow[t]{2}{*}{$\Delta M W_{\mathrm{det}}[\mathrm{g} / \mathrm{mol}](\%)$} & $\pm 10(5)$ & {$\left[(\mathrm{CpLi})_{2}(\mathrm{THF})_{3}\right]$} & 360 & 76 \\
\hline & & {$\left[(\mathrm{CpLi})_{2}(\mathrm{THF})_{4}\right]$} & 432 & 111 \\
\hline \multirow[t]{2}{*}{${ }^{1} \mathrm{H}-\mathrm{DOSY}$} & & & & $25^{\circ} \mathrm{C}$ \\
\hline & & Aggregate & $M W_{\text {calc }}[\mathrm{g} / \mathrm{mol}]$ & $M W_{\text {dif }}[\%]$ \\
\hline$D_{\mathrm{x}}\left[\mathrm{m}^{2} / \mathrm{s}\right]$ & $1.407 \mathrm{E}-09$ & [CpLi(THF)] & 144 & -34 \\
\hline $\log \left(D_{\mathbf{x}}\right)$ & -8.8517 & {$\left[\mathrm{CpLi}(\mathrm{THF})_{2}\right]$} & 216 & -1 \\
\hline $\log \left(D_{x, \text { norm }}\right)$ & -8.9012 & {$\left[\mathrm{CpLi}(\mathrm{THF})_{3}\right]$} & 288 & 32 \\
\hline$D_{\text {ref }}(\mathrm{TPhN})\left[\mathrm{m}^{2} / \mathrm{s}\right]$ & $8.791 \mathrm{E}-09$ & {$\left[\mathrm{CpLi}(\mathrm{THF})_{4}\right]$} & 360 & 65 \\
\hline $\log \left(D_{\text {ref }}\right)(\mathrm{TPhN})$ & -9.0559 & {$\left[(\mathrm{CpLi})_{2}(\mathrm{THF})\right]$} & 216 & -1 \\
\hline$M W_{\text {det }}[\mathrm{g} / \mathrm{mol}]$ & 218 & {$\left[(\mathrm{CpLi})_{2}(\mathrm{THF})_{2}\right]$} & 288 & 32 \\
\hline \multirow[t]{2}{*}{$\Delta M W_{\text {det }}[\mathrm{g} / \mathrm{mol}](\%)$} & $\pm 11(5)$ & {$\left[(\mathrm{CpLi})_{2}(\mathrm{THF})_{3}\right]$} & 360 & 65 \\
\hline & & {$\left[(\mathrm{CpLi})_{2}(\mathrm{THF})_{4}\right]$} & 432 & 98 \\
\hline \multirow[t]{2}{*}{${ }^{1} \mathrm{H}$-DOSY } & & & & $-50^{\circ} \mathrm{C}$ \\
\hline & & Aggregate & $M W_{\text {calc }}[\mathrm{g} / \mathrm{mol}]$ & $M W_{\text {dif }}[\%]$ \\
\hline$D_{\mathrm{x}}\left[\mathrm{m}^{2} / \mathrm{s}\right]$ & $3.381 \mathrm{E}-10$ & [CpLi(THF)] & 144 & -35 \\
\hline $\log \left(D_{\mathbf{x}}\right)$ & -9.4710 & {$\left[\mathrm{CpLi}(\mathrm{THF})_{2}\right]$} & 216 & -2 \\
\hline $\log \left(D_{\mathrm{x}, \text { norm }}\right)$ & -8.9050 & {$\left[\mathrm{CpLi}(\mathrm{THF})_{3}\right]$} & 288 & 30 \\
\hline$D_{\text {ref }}(\mathrm{TMB})\left[\mathrm{m}^{2} / \mathrm{s}\right]$ & $4.562 \mathrm{E}-10$ & {$\left[\mathrm{CpLi}(\mathrm{THF})_{4}\right]$} & 360 & 63 \\
\hline $\log \left(D_{\text {ref }}\right)($ TMB $)$ & -9.3408 & {$\left[(\mathrm{CpLi})_{2}(\mathrm{THF})\right]$} & 216 & -2 \\
\hline$M W_{\text {det }}[\mathrm{g} / \mathrm{mol}]$ & 222 & {$\left[(\mathrm{CpLi})_{2}(\mathrm{THF})_{2}\right]$} & 288 & 30 \\
\hline \multirow[t]{2}{*}{$\Delta M W_{\mathrm{det}}[\mathrm{g} / \mathrm{mol}](\%)$} & $\pm 11(5)$ & {$\left[(\mathrm{CpLi})_{2}(\mathrm{THF})_{3}\right]$} & 360 & 63 \\
\hline & & {$\left[(\mathrm{CpLi})_{2}(\mathrm{THF})_{4}\right]$} & 432 & 95 \\
\hline \multirow[t]{2}{*}{${ }^{1} \mathrm{H}-\mathrm{DOSY}$} & & & & $-80^{\circ} \mathrm{C}$ \\
\hline & & Aggregate & $M W_{\text {calc }}[\mathrm{g} / \mathrm{mol}]$ & $M W_{\text {dif }}[\%]$ \\
\hline$D_{\mathbf{x}}\left[\mathrm{m}^{2} / \mathrm{s}\right]$ & $1.144 \mathrm{E}-10$ & [CpLi(THF)] & 144 & -36 \\
\hline $\log \left(D_{\mathbf{x}}\right)$ & -9.9416 & {$\left[\mathrm{CpLi}(\mathrm{THF})_{2}\right]$} & 216 & -4 \\
\hline $\log \left(D_{\mathrm{x}, \text { norm }}\right)$ & -8.9090 & {$\left[\mathrm{CpLi}(\mathrm{THF})_{3}\right]$} & 288 & 28 \\
\hline$D_{\text {ref }}(\mathrm{TMB})\left[\mathrm{m}^{2} / \mathrm{s}\right]$ & $1.558 \mathrm{E}-10$ & {$\left[\mathrm{CpLi}(\mathrm{THF})_{4}\right]$} & 360 & 60 \\
\hline $\log \left(D_{\text {ref }}\right)($ TMB $)$ & -9.8074 & {$\left[(\mathrm{CpLi})_{2}(\mathrm{THF})\right]$} & 216 & -4 \\
\hline$M W_{\text {det }}[\mathrm{g} / \mathrm{mol}]$ & 225 & {$\left[(\mathrm{CpLi})_{2}(\mathrm{THF})_{2}\right]$} & 288 & 28 \\
\hline \multirow[t]{2}{*}{$\Delta M W_{\text {det }}[\mathrm{g} / \mathrm{mol}](\%)$} & $\pm 11(5)$ & {$\left[(\mathrm{CpLi})_{2}(\mathrm{THF})_{3}\right]$} & 360 & 60 \\
\hline & & {$\left[(\mathrm{CpLi})_{2}(\mathrm{THF})_{4}\right]$} & 432 & 92 \\
\hline${ }^{1} \mathrm{H}-\mathrm{DOSY}$ & & & & $-100^{\circ} \mathrm{C}$ \\
\hline No interpretable data & & & & \\
\hline
\end{tabular}




\begin{tabular}{|c|c|c|c|c|}
\hline \multicolumn{4}{|l|}{${ }^{7} \mathrm{Li}-\mathrm{DOSY}$} & \multirow{2}{*}{$\begin{array}{l}50^{\circ} \mathrm{C} \\
M W_{\text {dif }}[\%]\end{array}$} \\
\hline & & Aggregate & $M W_{\text {calc }}[\mathrm{g} / \mathrm{mol}]$ & \\
\hline$D_{\mathbf{x}}\left[\mathrm{m}^{2} / \mathrm{s}\right]$ & $2.085 \mathrm{E}-09$ & {$[\mathrm{CpLi}(\mathrm{THF})]$} & 144 & -35 \\
\hline $\log \left(D_{x}\right)$ & -8.6809 & {$\left[\mathrm{CpLi}(\mathrm{THF})_{2}\right]$} & 216 & -2 \\
\hline $\log \left(D_{x, \text { norm }}\right)$ & -8.9039 & {$\left[\mathrm{CpLi}(\mathrm{THF})_{3}\right]$} & 288 & 31 \\
\hline$D_{\text {ref }}(\mathrm{TMB})\left[\mathrm{m}^{2} / \mathrm{s}\right]$ & $2.806 \mathrm{E}-09$ & {$\left[\mathrm{CpLi}(\mathrm{THF})_{4}\right]$} & 360 & 63 \\
\hline $\log \left(D_{\text {ref }}\right)($ TMB $)$ & -8.5519 & {$\left[(\mathrm{CpLi})_{2}(\mathrm{THF})\right]$} & 216 & -2 \\
\hline$M W_{\text {det }}[\mathrm{g} / \mathrm{mol}]$ & 221 & {$\left[(\mathrm{CpLi})_{2}(\mathrm{THF})_{2}\right]$} & 288 & 31 \\
\hline \multirow{2}{*}{$\Delta M W_{\text {det }}[\mathrm{g} / \mathrm{mol}](\%)$} & $\pm 11(5)$ & {$\left[(\mathrm{CpLi})_{2}(\mathrm{THF})_{3}\right]$} & 360 & 63 \\
\hline & & {$\left[(\mathrm{CpLi})_{2}(\mathrm{THF})_{4}\right]$} & 432 & 96 \\
\hline \multirow[t]{2}{*}{${ }^{7} \mathbf{L i}$-DOSY } & & & & $25^{\circ} \mathrm{C}$ \\
\hline & & Aggregate & $M W_{\text {calc }}[\mathrm{g} / \mathrm{mol}]$ & $M W_{\mathrm{dif}}[\%]$ \\
\hline$D_{\mathbf{x}}\left[\mathrm{m}^{2} / \mathrm{s}\right]$ & $1.519 \mathrm{E}-09$ & {$[\mathrm{CpLi}(\mathrm{THF})]$} & 144 & -30 \\
\hline $\log \left(D_{x}\right)$ & -8.8184 & {$\left[\mathrm{CpLi}(\mathrm{THF})_{2}\right]$} & 216 & 5 \\
\hline $\log \left(D_{x, \text { norm }}\right)$ & -8.8874 & {$\left[\mathrm{CpLi}(\mathrm{THF})_{3}\right]$} & 288 & 40 \\
\hline$D_{\text {ref }}(\mathrm{TMB})\left[\mathrm{m}^{2} / \mathrm{s}\right]$ & $1.968 \mathrm{E}-09$ & {$\left[\mathrm{CpLi}(\mathrm{THF})_{4}\right]$} & 360 & 74 \\
\hline $\log \left(D_{\text {ref }}\right)($ TMB $)$ & -8.7060 & {$\left[(\mathrm{CpLi})_{2}(\mathrm{THF})\right]$} & 216 & 5 \\
\hline$M W_{\text {det }}[\mathrm{g} / \mathrm{mol}]$ & 207 & {$\left[(\mathrm{CpLi})_{2}(\mathrm{THF})_{2}\right]$} & 288 & 40 \\
\hline \multirow[t]{2}{*}{$\Delta M W_{\text {det }}[\mathrm{g} / \mathrm{mol}](\%)$} & $\pm 10(5)$ & {$\left[(\mathrm{CpLi})_{2}(\mathrm{THF})_{3}\right]$} & 360 & 74 \\
\hline & & {$\left[(\mathrm{CpLi})_{2}(\mathrm{THF})_{4}\right]$} & 432 & 109 \\
\hline \multirow[t]{2}{*}{${ }^{7} \mathrm{Li}-\mathrm{DOSY}$} & & & & $-50^{\circ} \mathrm{C}$ \\
\hline & & Aggregate & $M W_{\text {calc }}[\mathrm{g} / \mathrm{mol}]$ & $M W_{\text {dif }}[\%]$ \\
\hline$D_{\mathbf{x}}\left[\mathrm{m}^{2} / \mathrm{s}\right]$ & $3.369 \mathrm{E}-10$ & {$[\mathrm{CpLi}(\mathrm{THF})]$} & 144 & -35 \\
\hline $\log \left(D_{\mathbf{x}}\right)$ & -9.4724 & {$\left[\mathrm{CpLi}(\mathrm{THF})_{2}\right]$} & 216 & -3 \\
\hline $\log \left(D_{x, \text { norm }}\right)$ & -8.9066 & {$\left[\mathrm{CpLi}(\mathrm{THF})_{3}\right]$} & 288 & 29 \\
\hline$D_{\text {ref }}(\mathrm{TMB})\left[\mathrm{m}^{2} / \mathrm{s}\right]$ & $4.562 \mathrm{E}-10$ & {$\left[\mathrm{CpLi}(\mathrm{THF})_{4}\right]$} & 360 & 62 \\
\hline $\log \left(D_{\text {ref }}\right)(\mathrm{TMB})$ & -9.3408 & {$\left[(\mathrm{CpLi})_{2}(\mathrm{THF})\right]$} & 216 & -3 \\
\hline$M W_{\text {det }}[\mathrm{g} / \mathrm{mol}]$ & 223 & {$\left[(\mathrm{CpLi})_{2}(\mathrm{THF})_{2}\right]$} & 288 & 29 \\
\hline \multirow[t]{2}{*}{$\Delta M W_{\text {det }}[\mathrm{g} / \mathrm{mol}](\%)$} & $\pm 11(5)$ & {$\left[(\mathrm{CpLi})_{2}(\mathrm{THF})_{3}\right]$} & 360 & 62 \\
\hline & & {$\left[(\mathrm{CpLi})_{2}(\mathrm{THF})_{4}\right]$} & 432 & 94 \\
\hline \multicolumn{4}{|l|}{${ }^{7} \mathrm{Li}-\mathrm{DOSY}$} & $-80^{\circ} \mathrm{C} \&-100^{\circ} \mathrm{C}$ \\
\hline \multicolumn{5}{|l|}{ No interpretable data } \\
\hline \multicolumn{5}{|c|}{$\begin{array}{l}\text { Table 5-27: }{ }^{1} \mathrm{H}-\text { and }{ }^{7} \mathrm{Li}-\mathrm{DOSY}-\mathrm{ECC}-M W \text { estimation of CpLi in DMSO- } d_{6}(15 \mathrm{mM}) \text { at } 25^{\circ} \mathrm{C} \text {. TMB was used as internal } \\
\text { reference with } \log \left(D_{\text {reffix }}\right)(\mathrm{TMB})=-9.2963 \text {. The accuracy of the ECC }{ }^{\mathrm{DMSO}} \text { (merged) is in the range of } M W_{\mathrm{dif}}= \pm 20 \% \text {. } \\
\text { Hypothetical, even though unlikely, aggregates are listed; other contributions are probably hindering diffusion. For the } \\
{ }^{7} \mathrm{Li}-\mathrm{DOSY} \text { only considering ECC- } M W \text { estimation leads to no viable structure. For ECC }{ }^{\mathrm{DMSO}} \text { parameters see Figure } 5-1 \text {. }\end{array}$} \\
\hline \multirow[t]{2}{*}{${ }^{1} \mathrm{H}-\mathrm{DOSY}$} & & & & $25^{\circ} \mathrm{C}$ \\
\hline & & Aggregate & $M W_{\text {calc }}[\mathrm{g} / \mathrm{mol}]$ & $M W_{\text {dif }}[\%]$ \\
\hline$D_{\mathrm{x}}\left[\mathrm{m}^{2} / \mathrm{s}\right]$ & $1.444 \mathrm{E}-10$ & {$[\mathrm{Cp}]^{-}$} & 65 & -47 \\
\hline $\log \left(D_{\mathbf{x}}\right)$ & -9.8404 & {$[\mathrm{CpLi}]$} & 72 & -41 \\
\hline $\log \left(D_{x, \text { norm }}\right)$ & -9.2763 & [CpLi(DMSO)] & 150 & 23 \\
\hline$D_{\text {ref }}(\mathrm{TMB})\left[\mathrm{m}^{2} / \mathrm{s}\right]$ & $1.379 \mathrm{E}-10$ & & & \\
\hline $\log \left(D_{\text {ref }}\right)(\mathrm{TMB})$ & -9.8604 & & & \\
\hline$M W_{\text {det }}[\mathrm{g} / \mathrm{mol}]$ & 123 & & & \\
\hline$\Delta M W_{\text {det }}[\mathrm{g} / \mathrm{mol}](\%)$ & $\pm 10(8)$ & & & \\
\hline
\end{tabular}




\begin{tabular}{|c|c|c|c|c|}
\hline${ }^{7} \mathrm{Li}-\mathrm{DOSY}$ & & & & $25^{\circ} \mathrm{C}$ \\
\hline & & Aggregate & $M W_{\text {calc }}[\mathrm{g} / \mathrm{mol}]$ & $M W_{\text {dif }}[\%]$ \\
\hline$D_{\mathrm{x}}\left[\mathrm{m}^{2} / \mathrm{s}\right]$ & $2.853 \mathrm{E}-10$ & {$[\mathrm{CpLi}]$} & 72 & 67 \\
\hline $\log \left(D_{x}\right)$ & -9.5447 & & & \\
\hline $\log \left(D_{\mathrm{x}, \text { norm }}\right)$ & -8.9039 & & & \\
\hline$D_{\text {ref }}(\mathrm{TMB})\left[\mathrm{m}^{2} / \mathrm{s}\right]$ & $1.379 \mathrm{E}-10$ & & & \\
\hline $\log \left(D_{\text {ref }}\right)($ TMB $)$ & -9.8604 & & & \\
\hline$M W_{\text {det }}[\mathrm{g} / \mathrm{mol}]$ & 43 & & & \\
\hline$\Delta M W_{\text {det }}[\mathrm{g} / \mathrm{mol}](\%)$ & $\pm 3(6)$ & & & \\
\hline
\end{tabular}

\section{ECC-MW Estimation of CpNa in THF and DMSO}

Table 5-28: ${ }^{1} \mathrm{H}$-DOSY-ECC- $M W$ estimation of CpNa in THF- $d_{8}(15 \mathrm{mM})$ at different temperatures. TPhN or TMB were used as internal references with $\log \left(D_{\text {ref,fix }}\right)(\mathrm{TPhN})=-9.1054$ or $\log \left(D_{\text {ref,fix }}\right)(\mathrm{TMB})=-8.7749$. The accuracy of the ECC $\mathrm{EC}^{\mathrm{THF}}$ (DSE) is in the range of $M W_{\text {dif }}= \pm 8 \% \cdot{ }^{[109 a]}$ Hypothetical aggregates are $\left[\mathrm{CpNa}(\mathrm{THF})_{\mathrm{x}}\right]$ and $\left[(\mathrm{CpNa})_{2}(\mathrm{THF})_{\mathrm{x}}\right]$ with $\mathrm{x}=1-4$. For ECC ${ }^{\mathrm{THF}}$ parameters and changes compared to literature ${ }^{[109 \mathrm{a}]}$ see section 2.1.1.

\begin{tabular}{|c|c|c|c|c|}
\hline${ }^{1} \mathrm{H}-\mathrm{DOSY}$ & & & & $25^{\circ} \mathrm{C}$ \\
\hline & & Aggregate & $M W_{\text {calc }}[\mathrm{g} / \mathrm{mol}]$ & $M W_{\text {dif }}[\%]$ \\
\hline$D_{\mathbf{x}}\left[\mathrm{m}^{2} / \mathrm{s}\right]$ & $1.120 \mathrm{E}-09$ & {$[\mathrm{CpNa}(\mathrm{THF})]$} & 160 & -46 \\
\hline $\log \left(D_{\mathbf{x}}\right)$ & -8.9508 & {$\left[\mathrm{CpNa}(\mathrm{THF})_{2}\right]$} & 232 & -21 \\
\hline $\log \left(D_{x, \text { norm }}\right)$ & -8.9778 & {$\left[\mathrm{CpNa}(\mathrm{THF})_{3}\right]$} & 304 & 3 \\
\hline$D_{\text {ref }}(\mathrm{TPhN})\left[\mathrm{m}^{2} / \mathrm{s}\right]$ & $8.348 \mathrm{E}-09$ & {$\left[\mathrm{CpNa}(\mathrm{THF})_{4}\right]$} & 376 & 27 \\
\hline $\log \left(D_{\text {ref }}\right)(\mathrm{TPhN})$ & -9.0784 & {$\left[(\mathrm{CpNa})_{2}(\mathrm{THF})\right]$} & 248 & -16 \\
\hline$M W_{\text {det }}[\mathrm{g} / \mathrm{mol}]$ & 295 & {$\left[(\mathrm{CpNa})_{2}(\mathrm{THF})_{2}\right]$} & 320 & 8 \\
\hline \multirow[t]{2}{*}{$\Delta M W_{\text {det }}[\mathrm{g} / \mathrm{mol}](\%)$} & $\pm 16(5)$ & {$\left[(\mathrm{CpNa})_{2}(\mathrm{THF})_{3}\right]$} & 392 & 33 \\
\hline & & {$\left[(\mathrm{CpNa})_{2}(\mathrm{THF})_{4}\right]$} & 464 & 57 \\
\hline \multirow[t]{2}{*}{${ }^{1} \mathrm{H}-\mathrm{DOSY}$} & & & & $-50^{\circ} \mathrm{C}$ \\
\hline & & Aggregate & $M W_{\text {calc }}[\mathrm{g} / \mathrm{mol}]$ & $M W_{\text {dif }}[\%]$ \\
\hline$D_{\mathbf{x}}\left[\mathrm{m}^{2} / \mathrm{s}\right]$ & $2.817 \mathrm{E}-10$ & {$[\mathrm{CpNa}(\mathrm{THF})]$} & 160 & -44 \\
\hline $\log \left(D_{\mathbf{x}}\right)$ & -9.5502 & {$\left[\mathrm{CpNa}(\mathrm{THF})_{2}\right]$} & 232 & -19 \\
\hline $\log \left(D_{\mathrm{x}, \text { norm }}\right)$ & -8.9705 & {$\left[\mathrm{CpNa}(\mathrm{THF})_{3}\right]$} & 304 & 6 \\
\hline$D_{\text {ref }}(\mathrm{TMB})\left[\mathrm{m}^{2} / \mathrm{s}\right]$ & $4.420 \mathrm{E}-10$ & {$\left[\mathrm{CpNa}(\mathrm{THF})_{4}\right]$} & 376 & 31 \\
\hline $\log \left(D_{\text {ref }}\right)(\mathrm{TMB})$ & -9.3546 & {$\left[(\mathrm{CpNa})_{2}(\mathrm{THF})\right]$} & 248 & -14 \\
\hline$M W_{\text {det }}[\mathrm{g} / \mathrm{mol}]$ & 287 & {$\left[(\mathrm{CpNa})_{2}(\mathrm{THF})_{2}\right]$} & 320 & 12 \\
\hline \multirow[t]{2}{*}{$\Delta M W_{\text {det }}[\mathrm{g} / \mathrm{mol}](\%)$} & $\pm 15(5)$ & {$\left[(\mathrm{CpNa})_{2}(\mathrm{THF})_{3}\right]$} & 392 & 37 \\
\hline & & {$\left[(\mathrm{CpNa})_{2}(\mathrm{THF})_{4}\right]$} & 464 & 62 \\
\hline
\end{tabular}

Table 5-29: ${ }^{1} \mathrm{H}$-DOSY-ECC- $M W$ estimation of CpNa in DMSO- $d_{6}(15 \mathrm{mM})$ at different temperatures. TMB was used as internal reference with $\log \left(D_{\text {ref }, \text { fix }}\right)(\mathrm{TMB})=-9.2963$. The accuracy of the ECC $^{\text {DMSO }}$ (merged) is in the range of $M W_{\text {dif }}= \pm 20 \%$. Hypothetical, even though unlikely, aggregates are listed; other factors are probably hindering diffusion. For ECC ${ }^{\text {DMSO }}$ parameters see Figure 5-1.

\begin{tabular}{|c|c|c|c|c|}
\hline${ }^{1} \mathrm{H}-\mathrm{DOSY}$ & & & & $75^{\circ} \mathrm{C}$ \\
\hline & & Aggregate & $M W_{\text {calc }}[\mathrm{g} / \mathrm{mol}]$ & $M W_{\text {dif }}[\%]$ \\
\hline$D_{\mathbf{x}}\left[\mathrm{m}^{2} / \mathrm{s}\right]$ & $1.396 \mathrm{E}-09$ & {$[\mathrm{Cp}]^{-}$} & 65 & -51 \\
\hline $\log \left(D_{x}\right)$ & -8.8551 & {$[\mathrm{CpNa}]$} & 88 & -34 \\
\hline $\log \left(D_{\mathrm{x}, \text { norm }}\right)$ & -9.3003 & {$[\mathrm{CpNa}(\mathrm{DMSO})]$} & 166 & 25 \\
\hline$D_{\text {ref }}(\mathrm{TMB})\left[\mathrm{m}^{2} / \mathrm{s}\right]$ & $1.409 \mathrm{E}-09$ & & & \\
\hline $\log \left(D_{\text {ref }}\right)($ TMB $)$ & -8.8511 & & & \\
\hline$M W_{\text {det }}[\mathrm{g} / \mathrm{mol}]$ & 134 & & & \\
\hline$\Delta M W_{\operatorname{det}}[\mathrm{g} / \mathrm{mol}](\%)$ & $\pm 11(8)$ & & & \\
\hline
\end{tabular}




\begin{tabular}{|c|c|c|c|c|}
\hline${ }^{1} \mathrm{H}-\mathrm{DOSY}$ & & & & $50^{\circ} \mathrm{C}$ \\
\hline & & Aggregate & $M W_{\text {calc }}[\mathrm{g} / \mathrm{mol}]$ & $M W_{\text {dif }}[\%]$ \\
\hline$D_{\mathbf{x}}\left[\mathrm{m}^{2} / \mathrm{s}\right]$ & $8.695 \mathrm{E}-10$ & {$[\mathrm{Cp}]^{-}$} & 65 & -48 \\
\hline $\log \left(D_{\mathbf{x}}\right)$ & -9.0607 & {$[\mathrm{CpNa}]$} & 88 & -30 \\
\hline $\log \left(D_{\mathrm{x}, \text { norm }}\right)$ & -9.2835 & {$[\mathrm{CpNa}(\mathrm{DMSO})]$} & 166 & 32 \\
\hline$D_{\text {ref }}(\mathrm{TMB})\left[\mathrm{m}^{2} / \mathrm{s}\right]$ & $8.444 \mathrm{E}-10$ & & & \\
\hline $\log \left(D_{\text {ref }}\right)($ TMB $)$ & -9.0735 & & & \\
\hline$M W_{\text {det }}[\mathrm{g} / \mathrm{mol}]$ & 126 & & & \\
\hline$\Delta M W_{\text {det }}[\mathrm{g} / \mathrm{mol}](\%)$ & $\pm 10(8)$ & & & \\
\hline \multirow[t]{2}{*}{${ }^{1} \mathrm{H}-\mathrm{DOSY}$} & & & & $25^{\circ} \mathrm{C}$ \\
\hline & & Aggregate & $M W_{\text {calc }}[\mathrm{g} / \mathrm{mol}]$ & $M W_{\text {dif }}[\%]$ \\
\hline$D_{\mathbf{x}}\left[\mathrm{m}^{2} / \mathrm{s}\right]$ & $5.171 \mathrm{E}-10$ & {$[\mathrm{Cp}]^{-}$} & 65 & -47 \\
\hline $\log \left(D_{x}\right)$ & -9.2864 & {$[\mathrm{CpNa}]$} & 88 & -28 \\
\hline $\log \left(D_{\mathrm{x}, \text { norm }}\right)$ & -9.2733 & {$[\mathrm{CpNa}(\mathrm{DMSO})]$} & 166 & 37 \\
\hline$D_{\text {ref }}(\mathrm{TMB})\left[\mathrm{m}^{2} / \mathrm{s}\right]$ & $4.905 \mathrm{E}-10$ & & & \\
\hline $\log \left(D_{\text {ref }}\right)($ TMB $)$ & -9.3094 & & & \\
\hline$M W_{\text {det }}[\mathrm{g} / \mathrm{mol}]$ & 122 & & & \\
\hline$\Delta M W_{\text {det }}[\mathrm{g} / \mathrm{mol}](\%)$ & $\pm 10(8)$ & & & \\
\hline
\end{tabular}

\section{ECC-MW Estimation of CpK in THF and DMSO}

Table 5-30: ${ }^{1} \mathrm{H}$-DOSY-ECC- $M W$ estimation of CpK in THF- $d_{8}(15 \mathrm{mM})$ at $25^{\circ} \mathrm{C}$. TPhN was used as internal reference with $\log \left(D_{\text {reffix }}\right)(\mathrm{TPhN})=-9.1054$. The accuracy of the ECC ${ }^{\mathrm{THF}}(\mathrm{DSE})$ is in the range of $M W_{\mathrm{dif}}= \pm 8 \%$. Hypothetical aggregates are $\left[\mathrm{CpK}(\mathrm{THF})_{\mathrm{x}}\right]$ and $\left[(\mathrm{CpK})_{2}(\mathrm{THF})_{\mathrm{x}}\right]$ with $\mathrm{x}=1-4$. For $\mathrm{ECC}^{\mathrm{THF}}$ parameters and changes compared to literature ${ }^{[109 a]}$ see section 2.1.1.

\begin{tabular}{lrlll}
\hline${ }^{1} \mathbf{H}-D O S Y$ & & & & $\mathbf{2 5}^{\circ} \mathbf{C}$ \\
\hline & & Aggregate & $\boldsymbol{M} \boldsymbol{W}_{\text {calc }}[\mathbf{g} / \mathbf{m o l}]$ & $\boldsymbol{M W}_{\text {dif }}[\%]$ \\
$\boldsymbol{D}_{\mathbf{x}}\left[\mathbf{m}^{2} / \mathbf{s}\right]$ & $1.136 \mathrm{E}-09$ & {$[\mathrm{CpK}(\mathrm{THF})]$} & 176 & -47 \\
$\boldsymbol{l o g}\left(\boldsymbol{D}_{\mathbf{x}}\right)$ & -8.9446 & {$\left[\mathrm{CpK}(\mathrm{THF})_{2}\right]$} & 248 & -26 \\
$\boldsymbol{l o g}\left(\boldsymbol{D}_{\mathbf{x}, \mathbf{n o r m}}\right)$ & -9.0098 & {$\left[\mathrm{CpK}(\mathrm{THF})_{3}\right]$} & 320 & -4 \\
$\boldsymbol{D}_{\text {ref }}(\mathrm{TPhN})\left[\mathbf{m}^{2} / \mathbf{s}\right]$ & $9.116 \mathrm{E}-09$ & {$\left[\mathrm{CpK}(\mathrm{THF})_{4}\right]$} & 392 & 17 \\
$\boldsymbol{l o g}\left(\boldsymbol{D}_{\text {ref }}\right)(\mathbf{T P h N})$ & -9.0402 & {$\left[(\mathrm{CpK})_{2}(\mathrm{THF})\right]$} & 280 & -16 \\
$\boldsymbol{M} \boldsymbol{W}_{\text {det }}[\mathbf{g} / \mathbf{m o l}]$ & 335 & {$\left[(\mathrm{CpK})_{2}(\mathrm{THF})_{2}\right]$} & 353 & 5 \\
$\boldsymbol{\Delta} \boldsymbol{M} \boldsymbol{W}_{\text {det }}[\mathbf{g} / \mathbf{m o l}](\mathbf{\%})$ & $\pm 18(5)$ & {$\left[(\mathrm{CpK})_{2}(\mathrm{THF})_{3}\right]$} & 425 & 27 \\
& & {$\left[(\mathrm{CpK})_{2}(\mathrm{THF})_{4}\right]$} & 497 & 48 \\
\hline
\end{tabular}

Table 5-31: ${ }^{1} \mathrm{H}$-DOSY-ECC- $M W$ estimation of CpK in DMSO- $d_{6}(15 \mathrm{mM})$ at $25^{\circ} \mathrm{C}$. TMB was used as internal reference with $\log \left(D_{\text {reffix }}\right)($ TMB $)=-9.2963$. The accuracy of the $\mathrm{ECC}^{\text {DMSO }}$ (merged) is in the range of $M W_{\text {dif }}= \pm 20 \%$. Hypothetical, even though unlikely, aggregates are listed; other factors are probably hindering diffusion. For ECC ${ }^{\text {DMSO }}$ parameters see Figure 5-1.

\begin{tabular}{lrlll}
\hline${ }^{1} \mathrm{H}-\mathrm{DOSY}$ & & & & $\mathbf{2 5}^{\circ} \mathrm{C}$ \\
\hline & & Aggregate & $\boldsymbol{M} \boldsymbol{W}_{\text {calc }}[\mathbf{g} / \mathbf{m o l}]$ & $\boldsymbol{M} \boldsymbol{W}_{\text {dif }}[\%]$ \\
$\boldsymbol{D}_{\mathbf{x}}\left[\mathbf{m}^{2} / \mathbf{s}\right]$ & $5.304 \mathrm{E}-10$ & {$[\mathrm{Cp}]^{-}$} & 65 & -46 \\
$\log \left(\boldsymbol{D}_{\mathbf{x}}\right)$ & -9.2754 & {$[\mathrm{CpK}]$} & 104 & -14 \\
$\log \left(\boldsymbol{D}_{\mathbf{x}, \text { norm }}\right)$ & -9.2706 & {$[\mathrm{CpK}(\mathrm{DMSO})]$} & 182 & 52 \\
$\boldsymbol{D}_{\text {ref }}(\mathrm{TMB})\left[\mathbf{m}^{2} / \mathbf{s}\right]$ & $4.999 \mathrm{E}-10$ & & \\
$\log \left(\boldsymbol{D}_{\text {ref }}\right)(\mathrm{TMB})$ & -9.3011 & & \\
$\boldsymbol{M} \boldsymbol{W}_{\text {det }}[\mathbf{g} / \mathbf{m o l}]$ & 121 & & \\
$\Delta \boldsymbol{M} \boldsymbol{W}_{\text {det }}[\mathrm{g} / \mathbf{m o l}](\%)$ & $\pm 9(8)$ & & \\
\hline
\end{tabular}




\section{ECC-MW Estimation of $\mathrm{CpRb}$ in THF and DMSO}

Table 5-32: ${ }^{1} \mathrm{H}$-DOSY-ECC- $M W$ estimation of $\mathrm{CpRb}$ in THF- $d_{8}(15 \mathrm{mM})$ at $25^{\circ} \mathrm{C}$. TPhN was used as internal reference with $\log \left(D_{\text {ref }, \text { fix }}\right)(\mathrm{TPhN})=-9.1054$. The accuracy of the $\mathrm{ECC}^{\mathrm{THF}}(\mathrm{DSE})$ is in the range of $M W_{\text {dif }}= \pm 8 \%$. Hypothetical aggregates are $\left[\mathrm{CpRb}(\mathrm{THF})_{\mathrm{x}}\right]$ and $\left[(\mathrm{CpRb})_{2}(\mathrm{THF})_{\mathrm{x}}\right]$ with $\mathrm{x}=1-4$. For $\mathrm{ECC}^{\mathrm{THF}}$ parameters and changes compared to literature $^{[109 \mathrm{a}]}$ see section 2.1.1.

\begin{tabular}{|c|c|c|c|c|}
\hline${ }^{1} \mathrm{H}$-DOSY & & & & $25^{\circ} \mathrm{C}$ \\
\hline & & Aggregate & $M W_{\text {calc }}[\mathrm{g} / \mathrm{mol}]$ & $M W_{\text {dif }}[\%]$ \\
\hline$D_{\mathbf{x}}\left[\mathbf{m}^{2} / \mathbf{s}\right]$ & $1.272 \mathrm{E}-09$ & {$[\mathrm{CpRb}(\mathrm{THF})]$} & 223 & -24 \\
\hline $\log \left(D_{\mathbf{x}}\right)$ & -8.8955 & {$\left[\mathrm{CpRb}(\mathrm{THF})_{2}\right]$} & 295 & 0 \\
\hline $\log \left(D_{\mathrm{x}, \text { norm }}\right)$ & -8.9770 & {$\left[\mathrm{CpRb}(\mathrm{THF})_{3}\right]$} & 367 & 25 \\
\hline$D_{\text {ref }}(\mathrm{TPhN})\left[\mathrm{m}^{2} / \mathrm{s}\right]$ & $9.464 \mathrm{E}-09$ & {$\left[\mathrm{CpRb}(\mathrm{THF})_{4}\right]$} & 439 & 49 \\
\hline $\log \left(D_{\text {ref }}\right)(\mathrm{TPhN})$ & -9.0239 & {$\left[(\mathrm{CpRb})_{2}(\mathrm{THF})\right]$} & 373 & 27 \\
\hline$M W_{\text {det }}[\mathrm{g} / \mathrm{mol}]$ & 294 & {$\left[(\mathrm{CpRb})_{2}(\mathrm{THF})_{2}\right]$} & 445 & 51 \\
\hline \multirow[t]{2}{*}{$\Delta M W_{\text {det }}[\mathrm{g} / \mathrm{mol}](\%)$} & $\pm 16(5)$ & {$\left[(\mathrm{CpRb})_{2}(\mathrm{THF})_{3}\right]$} & 517 & 76 \\
\hline & & {$\left[(\mathrm{CpRb})_{2}(\mathrm{THF})_{4}\right]$} & 589 & 100 \\
\hline
\end{tabular}

Table 5-33: ${ }^{1} \mathrm{H}$-DOSY-ECC- $M W$ estimation of CpRb in DMSO- $d_{6}(15 \mathrm{mM})$ at $25^{\circ} \mathrm{C}$. TMB was used as internal reference with $\log \left(D_{\text {ref,fix }}\right)(\mathrm{TMB})=-9.2963$. The accuracy of the $\mathrm{ECC}^{\mathrm{DMSO}}$ (merged) is in the range of $M W_{\text {dif }}= \pm 20 \%$. Hypothetical, even though unlikely, aggregates are listed; other contributions could be hindering diffusion. For ECC ${ }^{\mathrm{DMSO}}$ parameters see Figure 5-1.

\begin{tabular}{lrlll}
\hline${ }^{1} \mathrm{H}-\mathrm{DOSY}$ & & & & $\mathbf{2 5}^{\circ} \mathrm{C}$ \\
\hline & & Aggregate & $\boldsymbol{M} \boldsymbol{W}_{\text {calc }}[\mathbf{g} / \mathbf{m o l}]$ & $\boldsymbol{M} \boldsymbol{W}_{\text {dif }}[\%]$ \\
$\boldsymbol{D}_{\mathbf{x}}\left[\mathbf{m}^{2} / \mathbf{s}\right]$ & $5.282 \mathrm{E}-10$ & {$[\mathrm{Cp}]^{-}$} & 65 & -45 \\
$\log \left(\boldsymbol{D}_{\mathbf{x}}\right)$ & -9.2772 & {$[\mathrm{CpRb}]$} & 151 & 27 \\
$\log \left(\boldsymbol{D}_{\mathbf{x}, \text { norm }}\right)$ & -9.2671 & {$[\mathrm{CpRb}(\mathrm{DMSO})]$} & 229 & 93 \\
$\boldsymbol{D}_{\text {ref }}(\mathrm{TMB})\left[\mathbf{m}^{2} / \mathbf{s}\right]$ & $4.938 \mathrm{E}-10$ & & \\
$\log \left(\boldsymbol{D}_{\text {ref }}\right)(\mathrm{TMB})$ & -9.3064 & & \\
$\boldsymbol{M} \boldsymbol{W}_{\text {det }}[\mathbf{g} / \mathbf{m o l}]$ & 119 & & \\
$\Delta \boldsymbol{M} \boldsymbol{W}_{\text {det }}[\mathrm{g} / \mathbf{m o l}](\%)$ & $\pm 9(8)$ & & \\
\hline
\end{tabular}

\section{ECC-MW Estimation of CpCs in THF and DMSO}

Table 5-34: ${ }^{1} \mathrm{H}$-DOSY-ECC- $M W$ estimation of CpCs in THF- $d_{8}(15 \mathrm{mM})$ at different temperatures. TPhN or TMB were used as internal references with $\log \left(D_{\text {reffix }}\right)(\mathrm{TPhN})=-9.1054$ or $\log \left(D_{\text {ref,fix }}\right)(\mathrm{TMB})=-8.7749$. The accuracy of the $\mathrm{ECC}^{\mathrm{THF}}$ (merged) is in the range of $M W_{\mathrm{dif}}= \pm 18 \%$. Exemplary, hypothetical aggregates are $\left[(\mathrm{CpCs})_{5}(\mathrm{THF})_{10}\right]$ or $\left[(\mathrm{CpCs})_{6}(\mathrm{THF})_{12}\right]$, while other combinations may also be viable. For $\mathrm{ECC}^{\mathrm{THF}}$ parameters and changes compared to literature ${ }^{[109 \mathrm{a}]}$ see section 2.1.1.

\begin{tabular}{|c|c|c|c|c|}
\hline${ }^{1} \mathrm{H}-\mathrm{DOSY}$ & & & & $50^{\circ} \mathrm{C}$ \\
\hline & & Aggregate & $M W_{\text {calc }}[\mathrm{g} / \mathrm{mol}]$ & $M W_{\text {dif }}[\%]$ \\
\hline$D_{\mathbf{x}}\left[\mathrm{m}^{2} / \mathrm{s}\right]$ & $6.201 \mathrm{E}-09$ & {$\left[(\mathrm{CpCs})_{5}(\mathrm{THF})_{10}\right]$} & 1710 & -8 \\
\hline $\log \left(D_{\mathbf{x}}\right)$ & -9.2076 & {$\left[(\mathrm{CpCs})_{6}(\mathrm{THF})_{12}\right]$} & 2053 & 10 \\
\hline $\log \left(D_{\mathrm{x}, \text { norm }}\right)$ & -9.4085 & & & \\
\hline$D_{\text {ref }}(\mathrm{TMB})\left[\mathrm{m}^{2} / \mathrm{s}\right]$ & $2.667 \mathrm{E}-09$ & & & \\
\hline $\log \left(D_{\text {ref }}\right)($ TMB $)$ & -8.5740 & & & \\
\hline$M W_{\text {det }}[\mathrm{g} / \mathrm{mol}]$ & 1864 & & & \\
\hline$\Delta M W_{\operatorname{det}}[\mathrm{g} / \mathrm{mol}](\%)$ & $\pm 199(11)$ & & & \\
\hline
\end{tabular}




\begin{tabular}{|c|c|c|c|c|}
\hline${ }^{1} \mathrm{H}-\mathrm{DOSY}$ & & & & $25^{\circ} \mathrm{C}$ \\
\hline & & Aggregate & $M W_{\text {calc }}[\mathrm{g} / \mathrm{mol}]$ & $M W_{\text {dif }}[\%]$ \\
\hline$D_{\mathbf{x}}\left[\mathrm{m}^{2} / \mathrm{s}\right]$ & $4.595 \mathrm{E}-09$ & {$\left[(\mathrm{CpCs})_{5}(\mathrm{THF})_{10}\right]$} & 1710 & -8 \\
\hline $\log \left(D_{x}\right)$ & -9.3377 & {$\left[(\mathrm{CpCs})_{6}(\mathrm{THF})_{12}\right]$} & 2053 & 11 \\
\hline $\log \left(D_{\mathrm{x}, \text { norm }}\right)$ & -9.4072 & & & \\
\hline$D_{\text {ref }}(\mathrm{TPhN})\left[\mathrm{m}^{2} / \mathrm{s}\right]$ & $9.211 \mathrm{E}-09$ & & & \\
\hline $\log \left(D_{\text {ref }}\right)(\mathrm{TPhN})$ & -9.0359 & & & \\
\hline$M W_{\operatorname{det}}[\mathrm{g} / \mathrm{mol}]$ & 1855 & & & \\
\hline$\Delta M W_{\text {det }}[\mathrm{g} / \mathrm{mol}](\%)$ & $\pm 198(11)$ & & & \\
\hline
\end{tabular}

Table 5-35: ${ }^{1} \mathrm{H}-\mathrm{DOSY}-\mathrm{ECC}-M W$ estimation of CpCs in DMSO- $d_{6}(15 \mathrm{mM})$ at $25^{\circ} \mathrm{C}$. TMB was used as internal reference with $\log \left(D_{\text {ref,fix }}\right)(\mathrm{TMB})=-9.2963$. The accuracy of the ECC ${ }^{\text {DMSO }}$ (merged) is in the range of $M W_{\mathrm{dif}}= \pm 20 \%$. Hypothetical, even though unlikely, aggregates are listed; other contributions are probably hindering diffusion. For ECC EMSO $^{\text {DM }}$ parameters see Figure 5-1.

\begin{tabular}{|c|c|c|c|c|}
\hline${ }^{1} \mathrm{H}-\mathrm{DOSY}$ & & & & $25^{\circ} \mathrm{C}$ \\
\hline & & Aggregate & $M W_{\text {calc }}[\mathrm{g} / \mathrm{mol}]$ & $M W_{\text {dif }}[\%]$ \\
\hline$D_{\mathbf{x}}\left[\mathrm{m}^{2} / \mathrm{s}\right]$ & $4.747 \mathrm{E}-10$ & {$[\mathrm{Cp}]^{-}$} & 65 & -62 \\
\hline $\log \left(D_{x}\right)$ & -9.3236 & {$[\mathrm{CpCs}]$} & 151 & -12 \\
\hline $\log \left(D_{\mathrm{x}, \text { norm }}\right)$ & -9.3699 & {$[\mathrm{CpCs}(\mathrm{DMSO})]$} & 276 & 62 \\
\hline$D_{\text {ref }}(\mathrm{TMB})\left[\mathrm{m}^{2} / \mathrm{s}\right]$ & $5.624 \mathrm{E}-10$ & & & \\
\hline $\log \left(D_{\text {ref }}\right)($ TMB $)$ & -9.2500 & & & \\
\hline$M W_{\text {det }}[\mathrm{g} / \mathrm{mol}]$ & 172 & & & \\
\hline$\Delta M W_{\text {det }}[\mathrm{g} / \mathrm{mol}](\%)$ & $\pm 15(8)$ & & & \\
\hline
\end{tabular}

\section{ECC-MW Estimation of MeCpLi in THF}

Table 5-36: ${ }^{1} \mathrm{H}$-DOSY-ECC- $M W$ estimation of MeCpLi in THF- $d_{8}(15 \mathrm{mM})$ at $25^{\circ} \mathrm{C}$. Adam was used as internal reference with $\log \left(D_{\text {ref, fix }}\right)($ Adam $)=-8.8078$. The accuracy of the ECC ${ }^{\mathrm{THF}}$ (DSE) is in the range of $M W_{\text {dif }}= \pm 8 \%$. Hypothetical aggregates are $\left[\mathrm{MeCpLi}(\mathrm{THF})_{\mathrm{x}}\right]$ and $\left[(\mathrm{MeCpLi})_{2}(\mathrm{THF})_{\mathrm{x}}\right]$ with $\mathrm{x}=1-4$. For ECC ${ }^{\mathrm{THF}}$ parameters and changes compared to literature ${ }^{[109 a]}$ see section 2.1.1.

\begin{tabular}{|c|c|c|c|c|}
\hline${ }^{1} \mathrm{H}-\mathrm{DOSY}$ & & & & $25^{\circ} \mathrm{C}$ \\
\hline & & Aggregate & $M W_{\text {calc }}[\mathrm{g} / \mathrm{mol}]$ & $M W_{\text {dif }}[\%]$ \\
\hline$D_{\mathrm{x}}\left[\mathrm{m}^{2} / \mathrm{s}\right]$ & $3.427 \mathrm{E}-10$ & [MeCpLi(THF)] & 157 & -34 \\
\hline $\log \left(D_{\mathbf{x}}\right)$ & -9.4650 & {$\left[\mathrm{MeCpLi}(\mathrm{THF})_{2}\right]$} & 229 & -3 \\
\hline $\log \left(D_{\mathbf{x}, \text { norm }}\right)$ & -8.9218 & {$\left[\mathrm{MeCpLi}(\mathrm{THF})_{3}\right]$} & 301 & 27 \\
\hline$D_{\text {ref }}($ Adam $)\left[\mathrm{m}^{2} / \mathrm{s}\right]$ & $4.432 \mathrm{E}-10$ & {$\left[\mathrm{MeCpLi}(\mathrm{THF})_{4}\right]$} & 373 & 57 \\
\hline $\log \left(D_{\text {ref }}\right)($ Adam $)$ & -9.3534 & {$\left[(\mathrm{MeCpLi})_{2}(\mathrm{THF})\right]$} & 242 & 2 \\
\hline$M W_{\text {det }}[\mathrm{g} / \mathrm{mol}]$ & 237 & {$\left[(\mathrm{MeCpLi})_{2}(\mathrm{THF})_{2}\right]$} & 314 & 32 \\
\hline \multirow[t]{2}{*}{$\Delta M W_{\operatorname{det}}[\mathrm{g} / \mathrm{mol}](\%)$} & $\pm 12(5)$ & {$\left[(\mathrm{MeCpLi})_{2}(\mathrm{THF})_{3}\right]$} & 386 & 63 \\
\hline & & {$\left[(\mathrm{MeCpLi})_{2}(\mathrm{THF})_{4}\right]$} & 458 & 93 \\
\hline
\end{tabular}




\section{ECC-MW Estimation of Cp ${ }^{\star} \mathrm{K}$ in THF}

Table 5-37: ${ }^{1} \mathrm{H}$-DOSY-ECC- $M W$ estimation of $\mathrm{Cp}^{*} \mathrm{~K}$ in THF- $d_{8}(15 \mathrm{mM})$ at $25^{\circ} \mathrm{C}$. TPhN was used as internal reference with $\log \left(D_{\text {reffix }}\right)(\mathrm{TPhN})=-9.1054$. The accuracy of the $\mathrm{ECC}^{\mathrm{THF}}(\mathrm{DSE})$ is in the range of $M W_{\mathrm{dif}}= \pm 8 \%$. Hypothetical aggregates are $\left[C \mathrm{p}^{*} \mathrm{~K}(\mathrm{THF})_{\mathrm{x}}\right]$ and $\left[\left(\mathrm{Cp}^{*} \mathrm{~K}\right)_{2}(\mathrm{THF})_{\mathrm{x}}\right]$ with $\mathrm{x}=1-4$. For $\mathrm{ECC}^{\mathrm{THF}}$ parameters and changes compared to literature $^{[109 a]}$ see section 2.1.1.

\begin{tabular}{|c|c|c|c|c|}
\hline${ }^{1} \mathrm{H}-\mathrm{DOSY}$ & & & & $25^{\circ} \mathrm{C}$ \\
\hline & & Aggregate & $M W_{\text {calc }}[\mathrm{g} / \mathrm{mol}]$ & $M W_{\text {dif }}[\%]$ \\
\hline$D_{\mathrm{x}}\left[\mathrm{m}^{2} / \mathrm{s}\right]$ & $1.096 \mathrm{E}-09$ & {$\left[\mathrm{Cp}^{*} \mathrm{~K}(\mathrm{THF})\right]$} & 246 & -35 \\
\hline $\log \left(D_{\mathbf{x}}\right)$ & -8.9602 & {$\left[\mathrm{Cp}^{*} \mathrm{~K}(\mathrm{THF})_{2}\right]$} & 318 & -16 \\
\hline $\log \left(D_{\mathrm{x}, \text { norm }}\right)$ & -9.0393 & {$\left[\mathrm{Cp}^{*} \mathrm{~K}(\mathrm{THF})_{3}\right]$} & 391 & 4 \\
\hline$D_{\text {ref }}(\mathrm{TPhN})\left[\mathrm{m}^{2} / \mathrm{s}\right]$ & $9.414 \mathrm{E}-09$ & {$\left[\mathrm{Cp}^{\star} \mathrm{K}(\mathrm{THF})_{4}\right]$} & 463 & 23 \\
\hline $\log \left(D_{\text {ref }}\right)(\mathrm{TPhN})$ & -9.0262 & {$\left[\left(\mathrm{Cp}^{\star} \mathrm{K}\right)_{2}(\mathrm{THF})\right]$} & 421 & 12 \\
\hline$M W_{\text {det }}[\mathrm{g} / \mathrm{mol}]$ & 377 & {$\left[\left(\mathrm{Cp}^{\star} \mathrm{K}\right)_{2}(\mathrm{THF})_{2}\right]$} & 493 & 31 \\
\hline \multirow[t]{2}{*}{$\Delta M W_{\text {det }}[\mathrm{g} / \mathrm{mol}](\%)$} & $\pm 21(5)$ & {$\left[\left(\mathrm{Cp}^{\star} \mathrm{K}\right)_{2}(\mathrm{THF})_{3}\right]$} & 565 & 50 \\
\hline & & {$\left[\left(\mathrm{Cp}^{*} \mathrm{~K}\right)_{2}(\mathrm{THF})_{4}\right]$} & 637 & 69 \\
\hline
\end{tabular}

\section{ECC-MW Estimation of $\mathrm{Cp}{ }^{*} \mathrm{Cs}$ in THF}

Table 5-38: ${ }^{1} \mathrm{H}$-DOSY-ECC- $M W$ estimation of $\mathrm{Cp}^{*} \mathrm{Cs}$ in THF- $d_{8}(15 \mathrm{mM})$ at $25^{\circ} \mathrm{C}$. TPhN was used as internal reference with $\log \left(D_{\text {ref,fix }}\right)(\mathrm{TPhN})=-9.1054$. The accuracy of the $\mathrm{ECC}^{\mathrm{THF}}(\mathrm{DSE})$ is in the range of $M W_{\mathrm{dif}}= \pm 8 \%$. Hypothetical aggregates are $\left[\mathrm{Cp}^{\star} \mathrm{Cs}(\mathrm{THF})_{\mathrm{x}}\right]$ and $\left[\left(\mathrm{Cp}^{\star} \mathrm{Cs}\right)_{2}(\mathrm{THF})_{\mathrm{x}}\right]$ with $\mathrm{x}=1-4$. For $\mathrm{ECC}^{\mathrm{THF}}$ parameters and changes compared to literature ${ }^{[109 \mathrm{a}]}$ see section 2.1.1.

\begin{tabular}{|c|c|c|c|c|}
\hline${ }^{1} \mathrm{H}-\mathrm{DOSY}$ & & & & $25^{\circ} \mathrm{C}$ \\
\hline & & Aggregate & $M W_{\text {calc }}[\mathrm{g} / \mathrm{mol}]$ & $M W_{\text {dif }}[\%]$ \\
\hline$D_{\mathrm{x}}\left[\mathrm{m}^{2} / \mathrm{s}\right]$ & $9.218 \mathrm{E}-10$ & {$\left[\mathrm{Cp}^{*} \mathrm{Cs}(\mathrm{THF})\right]$} & 340 & -40 \\
\hline $\log \left(D_{\mathbf{x}}\right)$ & -9.0354 & {$\left[\mathrm{Cp}^{*} \mathrm{Cs}(\mathrm{THF})_{2}\right]$} & 412 & -27 \\
\hline $\log \left(D_{\mathrm{x}, \text { norm }}\right)$ & -9.1411 & {$\left[\mathrm{Cp}^{*} \mathrm{Cs}(\mathrm{THF})_{3}\right]$} & 484 & -14 \\
\hline$D_{\text {ref }}(\mathrm{TPhN})\left[\mathrm{m}^{2} / \mathrm{s}\right]$ & $1.001 \mathrm{E}-09$ & {$\left[\mathrm{Cp}^{*} \mathrm{Cs}(\mathrm{THF})_{4}\right]$} & 556 & -1 \\
\hline $\log \left(D_{\text {ref }}\right)(\mathrm{TPhN})$ & -8.9996 & {$\left[\left(\mathrm{Cp}^{*} \mathrm{Cs}\right)_{2}(\mathrm{THF})\right]$} & 608 & 8 \\
\hline$M W_{\operatorname{det}}[\mathrm{g} / \mathrm{mol}]$ & 564 & {$\left[\left(\mathrm{Cp}^{*} \mathrm{Cs}\right)_{2}(\mathrm{THF})_{2}\right]$} & 680 & 21 \\
\hline \multirow[t]{2}{*}{$\Delta M W_{\text {det }}[\mathrm{g} / \mathrm{mol}](\%)$} & $\pm 33(6)$ & {$\left[\left(\mathrm{Cp}^{*} \mathrm{Cs}\right)_{2}(\mathrm{THF})_{3}\right]$} & 752 & 33 \\
\hline & & {$\left[\left(\mathrm{Cp}^{\star} \mathrm{Cs}\right)_{2}(\mathrm{THF})_{4}\right]$} & 825 & 46 \\
\hline
\end{tabular}

\section{Molar Van-Der-Waals Densities $\left(M D_{\mathrm{w}}\right)$}

Table 5-39: $M D_{\mathrm{W}}$ for most likely solution state structures of CpM derivatives.

\begin{tabular}{lccccccc}
\hline Aggregate & $\begin{array}{c}\boldsymbol{M W} \\
{[\mathbf{g} / \mathbf{m o l}]}\end{array}$ & $\mathbf{C}$ & $\mathbf{H}$ & $\mathbf{O}$ & $\mathbf{M}^{\mathbf{a}}$ & $\begin{array}{c}\boldsymbol{M} \boldsymbol{D}_{\mathbf{W}} \\
{\left[\mathbf{g} /\left(\mathbf{m o l} \cdot \mathbf{m}^{3}\right)\right]}\end{array}$ & $\begin{array}{c}\boldsymbol{\Sigma} \boldsymbol{V}_{\mathbf{W}} \\
{\left[\mathbf{m}^{3}\right]}\end{array}$ \\
\hline$\left[\mathbf{C p L i}(\mathrm{THF})_{2}\right]$ & 216 & 13 & 21 & 2 & 1 & $4.92 \mathrm{E}+29$ & $4.39 \mathrm{E}-28$ \\
{$\left[\mathbf{C p N a}(\mathrm{THF})_{3}\right]$} & 304 & 17 & 29 & 3 & 1 & $5.03 \mathrm{E}+29$ & $6.05 \mathrm{E}-28$ \\
{$\left[\mathbf{C p K}(\mathbf{T H F})_{3}\right]$} & 321 & 17 & 29 & 3 & 1 & $4.99 \mathrm{E}+29$ & $6.43 \mathrm{E}-28$ \\
{$\left[\mathbf{C p R b}(\mathbf{T H F})_{2}\right]$} & 295 & 13 & 21 & 3 & 1 & $5.56 \mathrm{E}+29$ & $5.31 \mathrm{E}-28$ \\
{$\left[(\mathbf{C p C s})_{\mathbf{5}}(\mathbf{T H F})_{\mathbf{1 0}}\right]$} & 1711 & 65 & 105 & 10 & 5 & $5.87 \mathrm{E}+29$ & $2.92 \mathrm{E}-27$ \\
{$\left[\mathbf{M e C p L i}(\mathbf{T H F})_{2}\right]$} & 230 & 14 & 23 & 2 & 1 & $4.89 \mathrm{E}+29$ & $4.71 \mathrm{E}-28$ \\
{$\left[\mathbf{C} \mathbf{p}^{*} \mathbf{K}(\mathbf{T H F})_{3}\right]$} & 391 & 22 & 39 & 3 & 1 & $4.87 \mathrm{E}+29$ & $8.01 \mathrm{E}-28$ \\
{$\left[\mathbf{C} \mathbf{p}^{\star} \mathbf{C s}(\mathbf{T H F})_{4}\right]$} & 557 & 26 & 47 & 4 & 1 & $5.43 \mathrm{E}+29$ & $1.02 \mathrm{E}-27$ \\
\hline
\end{tabular}

\footnotetext{
${ }^{a}$ Always the corresponding alkali metal: $\mathrm{M}=\mathrm{Li}, \mathrm{Na}, \mathrm{K}, \mathrm{Rb}$ or $\mathrm{Cs}$
} 
ECC-MW Estimations of RMgX (R = Et, $\mathrm{Pr}, n \mathrm{Bu}, n \mathrm{Hex}, n \mathrm{Oct}, n \mathrm{Dec} ; \mathrm{X}=\mathrm{Cl}, \mathrm{Br})$

\section{ECC-MW Estimation of EtMgCl in THF}

Table 5-40: ${ }^{1} \mathrm{H}$-DOSY-ECC- $M W$ estimation of EtMgCl in THF- $d_{8}(25 \mathrm{mM})$ at $25^{\circ} \mathrm{C}$. TPhN was used as internal reference with $\log \left(D_{\text {reffix }}\right)(\mathrm{TPhN})=-9.1054$. The accuracy of the ECC $\mathrm{EHF}^{\mathrm{THL}}(\mathrm{R})$ is in the range of $M W_{\mathrm{dif}}= \pm 8 \%$ (see section 2.3.1.1). Hypothetical aggregates are $\left[\mathrm{EtMgCl}(\mathrm{THF})_{2}\right]$ and $[\mathrm{EtMgCl}(\mathrm{THF})]_{2}$. For $\mathrm{ECC}^{\mathrm{THF}}$ parameters see Figure 5-8.

\begin{tabular}{|c|c|c|c|c|}
\hline${ }^{1} \mathrm{H}-\mathrm{DOSY}$ & & & & $25^{\circ} \mathrm{C}$ \\
\hline & & Aggregate & $M W_{\text {calc }}[\mathrm{g} / \mathrm{mol}]$ & $M W_{\text {dif }}[\%]$ \\
\hline$D_{\mathbf{x}}\left[\mathrm{m}^{2} / \mathrm{s}\right]$ & $1.299 \mathrm{E}-09$ & {$\left[\mathrm{EtMgCl}(\mathrm{THF})_{2}\right]$} & 233 & 2 \\
\hline $\log \left(D_{x}\right)$ & -8.8864 & {$[\mathrm{EtMgCl}(\mathrm{THF})]_{2}$} & 322 & 41 \\
\hline $\log \left(D_{\mathrm{x}, \text { norm }}\right)$ & -8.9360 & & & \\
\hline$D_{\text {ref }}(\mathrm{TPhN})\left[\mathrm{m}^{2} / \mathrm{s}\right]$ & $8.794 \mathrm{E}-10$ & & & \\
\hline $\log \left(D_{\text {ref }}\right)(\mathrm{TPhN})$ & -9.0558 & & & \\
\hline$M W_{\text {det }}[\mathrm{g} / \mathrm{mol}]$ & 228 & & & \\
\hline$\Delta M W_{\text {det }}[\mathrm{g} / \mathrm{mol}](\%)$ & $\pm 8(4)$ & & & \\
\hline
\end{tabular}

\section{ECC-MW Estimation of $i \mathrm{PrMgCl}$ in THF}

Table 5-41: ${ }^{1} \mathrm{H}$-DOSY-ECC- $M W$ estimation of $i \mathrm{PrMgCl}$ in THF- $d_{8}(25 \mathrm{mM})$ at $25^{\circ} \mathrm{C}$. TPhN was used as internal reference with $\log \left(D_{\text {reffix }}\right)(\mathrm{TPhN})=-9.1054$. The accuracy of the $\mathrm{ECC}^{\mathrm{THF}}(\mathrm{DSE})$ is in the range of $M W_{\mathrm{dif}}= \pm 8 \%$. Hypothetical aggregates are $\left[i \operatorname{PrMgCl}(\mathrm{THF})_{2}\right]$ and $[i \operatorname{PrMgCl}(\mathrm{THF})]_{2}$. For ECC ${ }^{\mathrm{THF}}$ parameters and changes compared to literature ${ }^{[109 \mathrm{a}]}$ see section 2.1.1.

\begin{tabular}{|c|c|c|c|c|}
\hline${ }^{1} \mathrm{H}-\mathrm{DOSY}$ & & & & $25^{\circ} \mathrm{C}$ \\
\hline & & Aggregate & $M W_{\text {calc }}[\mathrm{g} / \mathrm{mol}]$ & $M W_{\text {dif }}[\%]$ \\
\hline$D_{\mathrm{x}}\left[\mathrm{m}^{2} / \mathrm{s}\right]$ & $1.243 \mathrm{E}-09$ & {$\left[i \operatorname{PrMgCl}(\mathrm{THF})_{2}\right]$} & 247 & -2 \\
\hline $\log \left(D_{\mathbf{x}}\right)$ & -8.9055 & {$[i \operatorname{PrMgCl}(\mathrm{THF})]_{2}$} & 350 & 39 \\
\hline $\log \left(D_{\mathrm{x}, \text { norm }}\right)$ & -8.9371 & & & \\
\hline$D_{\text {ref }}(\mathrm{TPhN})\left[\mathrm{m}^{2} / \mathrm{s}\right]$ & $8.436 \mathrm{E}-10$ & & & \\
\hline $\log \left(D_{\text {ref }}\right)(\mathrm{TPhN})$ & -9.0739 & & & \\
\hline$M W_{\text {det }}[\mathrm{g} / \mathrm{mol}]$ & 251 & & & \\
\hline$\Delta M W_{\operatorname{det}}[\mathrm{g} / \mathrm{mol}](\%)$ & $\pm 13(5)$ & & & \\
\hline
\end{tabular}

\section{ECC-MW Estimation of $n \mathrm{BuMgCl}$ in THF}

Table 5-42: ${ }^{1} \mathrm{H}$-DOSY-ECC- $M W$ estimation of $n \mathrm{BuMgCl}$ in THF- $d_{8}(25 \mathrm{mM})$ at $25^{\circ} \mathrm{C}$. TPhN was used as internal reference with $\log \left(D_{\text {reffix }}\right)(\mathrm{TPhN})=-9.1054$. The accuracy of the $\mathrm{ECC}^{\mathrm{THF}}(\mathrm{RL})$ is in the range of $M W_{\mathrm{dif}}= \pm 8 \%$ (see section 2.3.1.1). Hypothetical aggregates are $\left[n \mathrm{BuMgCl}(\mathrm{THF})_{2}\right]$ and $[n \mathrm{BuMgCl}(\mathrm{THF})]_{2}$. For $\mathrm{ECC}^{\mathrm{THF}}$ parameters see Figure 5-8.

\begin{tabular}{|c|c|c|c|c|}
\hline${ }^{1} \mathrm{H}-\mathrm{DOSY}$ & & & & $25^{\circ} \mathrm{C}$ \\
\hline & & Aggregate & $M W_{\text {calc }}[\mathrm{g} / \mathrm{mol}]$ & $M W_{\text {dif }}[\%]$ \\
\hline$D_{\mathrm{x}}\left[\mathrm{m}^{2} / \mathrm{s}\right]$ & $1.234 \mathrm{E}-09$ & {$\left[n \mathrm{BuMgCl}(\mathrm{THF})_{2}\right]$} & 261 & 2 \\
\hline $\log \left(D_{\mathbf{x}}\right)$ & -8.9088 & {$[n \mathrm{BuMgCl}(\mathrm{THF})]_{2}$} & 378 & 48 \\
\hline $\log \left(D_{x, \text { norm }}\right)$ & -8.9750 & & & \\
\hline$D_{\text {ref }}(\mathrm{TPhN})\left[\mathrm{m}^{2} / \mathrm{s}\right]$ & $9.137 \mathrm{E}-10$ & & & \\
\hline $\log \left(D_{\text {ref }}\right)(\mathrm{TPhN})$ & -9.0392 & & & \\
\hline$M W_{\text {det }}[\mathrm{g} / \mathrm{mol}]$ & 255 & & & \\
\hline$\Delta M W_{\text {det }}[\mathrm{g} / \mathrm{mol}](\%)$ & $\pm 9(4)$ & & & \\
\hline
\end{tabular}




\section{ECC-MW Estimation of $n \mathrm{HexMgCl}$ in THF}

Table 5-43: ${ }^{1} \mathrm{H}-\mathrm{DOSY}-\mathrm{ECC}-M W$ estimation of $n \mathrm{HexMgCl}$ in THF- $d_{8}(25 \mathrm{mM})$ at $25^{\circ} \mathrm{C}$. $\mathrm{TPhN}$ was used as internal reference with $\log \left(D_{\text {ref,fix }}\right)(\mathrm{TPhN})=-9.1054$. The accuracy of the $\mathrm{ECC}^{\mathrm{THF}}(\mathrm{RL})$ is in the range of $M W_{\mathrm{dif}}= \pm 8 \%$ (see section 2.3.1.1). Hypothetical aggregates are $\left[n \operatorname{HexMgCl}(\mathrm{THF})_{2}\right]$ and $[n \operatorname{HexMgCl}(\mathrm{THF})]_{2}$. For ECC ${ }^{\mathrm{THF}}$ parameters see Figure 5-8.

\begin{tabular}{lrlll}
\hline${ }^{1} \mathbf{H}-\mathrm{DOSY}$ & & & $\mathbf{2 5}^{\circ} \mathbf{C}$ \\
\hline & & Aggregate & $\boldsymbol{M} \boldsymbol{W}_{\text {calc }}[\mathbf{g} / \mathbf{m o l}]$ & $\boldsymbol{M} \boldsymbol{W}_{\text {dif }}[\%]$ \\
$\boldsymbol{D}_{\mathbf{x}}\left[\mathbf{m}^{2} / \mathbf{s}\right]$ & $1.100 \mathrm{E}-09$ & {$\left[n \mathrm{HexMgCl}(\mathrm{THF})_{2}\right]$} & 289 & 0 \\
$\log \left(\boldsymbol{D}_{\mathbf{x}}\right)$ & -8.9586 & {$[n \mathrm{HexMgCl}(\mathrm{THF})]_{2}$} & 434 & 51 \\
$\log \left(\boldsymbol{D}_{\mathbf{x}, \mathbf{n o r m})}\right)$ & -9.0173 & & \\
$\boldsymbol{D}_{\text {ref }}(\mathrm{TPhN})\left[\mathbf{m}^{2} / \mathbf{s}\right]$ & $8.980 \mathrm{E}-10$ & & \\
$\log \left(\boldsymbol{D}_{\text {ref }}\right)(\mathrm{TPhN})$ & -9.0467 & & \\
$\boldsymbol{M} \boldsymbol{W}_{\text {det }}[\mathbf{g} / \mathbf{m o l}]$ & 288 & & \\
$\Delta \boldsymbol{M} \boldsymbol{W}_{\text {det }}[\mathbf{g} / \mathbf{m o l}](\%)$ & $\pm 11(4)$ & & \\
& & & \\
\hline
\end{tabular}

\section{ECC-MW Estimation of $n$ OctMgCl in THF}

Table 5-44: ${ }^{1} \mathrm{H}$-DOSY-ECC- $M W$ estimation of $n \mathrm{OctMgCl}$ in THF- $d_{8}(25 \mathrm{mM})$ at $25^{\circ} \mathrm{C}$. TPhN was used as internal reference with $\log \left(D_{\text {ref,fix }}\right)(\mathrm{TPhN})=-9.1054$. The accuracy of the $\mathrm{ECC}^{\mathrm{THF}}(\mathrm{RL})$ is in the range of $M W_{\mathrm{dif}}= \pm 8 \%$ (see section 2.3.1.1). Hypothetical aggregates are $\left[n \mathrm{OctMgCl}(\mathrm{THF})_{2}\right]$ and $[n \mathrm{OctMgCl}(\mathrm{THF})]_{2}$. For ECC ${ }^{\mathrm{THF}}$ parameters see Figure 5-8.

\begin{tabular}{lrlll}
\hline${ }^{1} \mathbf{H}-\mathrm{DOSY}$ & & & $M W_{\text {calc }}[\mathrm{g} / \mathrm{mol}]$ & $M W_{\text {dif }}[\%]$ \\
\hline & & Aggregate & $\boldsymbol{M} \boldsymbol{W}_{\text {calc }}[\mathrm{g} / \mathbf{m o l}]$ & $\boldsymbol{M} \boldsymbol{W}_{\text {dif }}[\%]$ \\
$\boldsymbol{D}_{\mathbf{x}}\left[\mathbf{m}^{2} / \mathbf{s}\right]$ & $1.029 \mathrm{E}-09$ & {$[n \text { OctMgCl(THF })_{2}$} & 317 & 0 \\
$\log \left(\boldsymbol{D}_{\mathbf{x}}\right)$ & -8.9875 & {$[n \text { OctMgCl}(\mathrm{THF})]_{2}$} & 490 & 55 \\
$\log \left(\boldsymbol{D}_{\mathbf{x}, \mathbf{n o r m}}\right)$ & -9.0509 & & \\
$\boldsymbol{D}_{\text {ref }}(\mathrm{TPhN})\left[\mathbf{m}^{2} / \mathbf{s}\right]$ & $9.079 \mathrm{E}-10$ & & \\
$\log \left(\boldsymbol{D}_{\text {ref }}\right)(\mathrm{TPhN})$ & -9.0420 & & \\
$\boldsymbol{M} \boldsymbol{W}_{\text {det }}[\mathbf{g} / \mathbf{m o l}]$ & 317 & & \\
$\boldsymbol{M} \boldsymbol{W}_{\text {det }}[\mathbf{g} / \mathbf{m o l}](\%)$ & $\pm 12(4)$ & & \\
\end{tabular}

\section{ECC-MW Estimation of $n \mathrm{DecMgCl}$ in THF}

Table 5-45: ${ }^{1} \mathrm{H}-\mathrm{DOSY}-\mathrm{ECC}-M W$ estimation of $n$ DecMgCl in THF- $d_{8}(25 \mathrm{mM})$ at $25^{\circ} \mathrm{C}$. TPhN was used as internal reference with $\log \left(D_{\text {ref,fix }}\right)(\mathrm{TPhN})=-9.1054$. The accuracy of the $\mathrm{ECC}^{\mathrm{THF}}(\mathrm{RL})$ is in the range of $M W_{\mathrm{dif}}= \pm 8 \%$ (see section 2.3.1.1). Hypothetical aggregates are $\left[n \operatorname{DecMgCl}(\mathrm{THF})_{2}\right]$ and $[n \operatorname{DecMgCl}(\mathrm{THF})]_{2}$. For ECC ${ }^{\mathrm{THF}}$ parameters see Figure 5-8.

\begin{tabular}{lrlll}
\hline${ }^{1} \mathbf{H}-\mathrm{DOSY}$ & & & $\mathbf{2 5}^{\circ} \mathbf{C}$ \\
\hline & & Aggregate & $\boldsymbol{M} \boldsymbol{W}_{\text {calc }}[\mathbf{g} / \mathbf{m o l}]$ & $\boldsymbol{M} \boldsymbol{W}_{\text {dif }}[\%]$ \\
$\boldsymbol{D}_{\mathbf{x}}\left[\mathbf{m}^{2} / \mathbf{s}\right]$ & $9.055 \mathrm{E}-10$ & {$\left[n \operatorname{DecMgCl}(\mathrm{THF})_{2}\right]$} & 345 & -1 \\
$\log \left(\boldsymbol{D}_{\mathbf{x}}\right)$ & -9.0431 & {$[n \operatorname{DecMgCl}(\mathrm{THF})]_{2}$} & 546 & 57 \\
$\log \left(\boldsymbol{D}_{\mathbf{x}, \mathbf{n o r m}}\right)$ & -9.0837 & & \\
$\boldsymbol{D}_{\text {ref }}(\mathbf{T P h N})\left[\mathbf{m}^{2} / \mathbf{s}\right]$ & $8.613 \mathrm{E}-10$ & & \\
$\log \left(\boldsymbol{D}_{\text {ref }}\right)(\mathbf{T P h N})$ & -9.0648 & & \\
$\boldsymbol{M} \boldsymbol{W}_{\text {det }}[\mathbf{g} / \mathbf{m o l}]$ & 348 & & \\
$\Delta \boldsymbol{M} \boldsymbol{W}_{\text {det }}[\mathbf{g} / \mathbf{m o l}](\%)$ & $\pm 14(4)$ & & \\
& & & \\
\hline
\end{tabular}




\section{ECC-MW Estimation of $n \mathrm{BuMgBr}$ in THF}

Table 5-46: ${ }^{1} \mathrm{H}-\mathrm{DOSY}-\mathrm{ECC}-\mathrm{MW}$ estimation of $n \mathrm{BuMgBr}$ in THF- $d_{8}(25 \mathrm{mM})$ at $25^{\circ} \mathrm{C}$. TPhN was used as internal reference with $\log \left(D_{\text {reffix }}\right)(\mathrm{TPhN})=-9.1054$. The accuracy of the ECC $^{\mathrm{THF}}(\mathrm{RLBr})$ is in the range of $\Delta M W= \pm 9 \%$ (theoretical; see section 2.3.1.1). Hypothetical aggregates are $\left[n \operatorname{BuMgBr}(\mathrm{THF})_{2}\right]$ and $[n \mathrm{BuMgBr}(\mathrm{THF})]_{2}$. For $\mathrm{ECC}^{\mathrm{THF}}$ parameters see Figure 5-8.

\begin{tabular}{|c|c|c|c|c|}
\hline${ }^{1} \mathrm{H}-\mathrm{DOSY}$ & & & & $25^{\circ} \mathrm{C}$ \\
\hline & & Aggregate & $M W_{\text {calc }}[\mathrm{g} / \mathrm{mol}]$ & $M W_{\text {dif }}[\%]$ \\
\hline$D_{\mathrm{x}}\left[\mathrm{m}^{2} / \mathrm{s}\right]$ & $1.222 \mathrm{E}-09$ & {$\left[n \mathrm{BuMgBr}(\mathrm{THF})_{2}\right]$} & 305 & -8 \\
\hline $\log \left(D_{x}\right)$ & -8.9129 & {$[n \mathrm{BuMgBr}(\mathrm{THF})]_{2}$} & 466 & 41 \\
\hline $\log \left(D_{\mathrm{x}, \text { norm }}\right)$ & -8.9660 & & & \\
\hline$D_{\text {ref }}(\mathrm{TPhN})\left[\mathrm{m}^{2} / \mathrm{s}\right]$ & $8.865 \mathrm{E}-10$ & & & \\
\hline $\log \left(D_{\text {ref }}\right)(\mathrm{TPhN})$ & -9.0523 & & & \\
\hline$M W_{\text {det }}[\mathrm{g} / \mathrm{mol}]$ & 330 & & & \\
\hline$\Delta M W_{\text {det }}[\mathrm{g} / \mathrm{mol}](\%)$ & $\pm 33(10)$ & & & \\
\hline
\end{tabular}

\section{Molar Van-Der-Waals Densities $\left(M D_{w}\right)$}

Table 5-47: $M D_{\mathrm{W}}$ for most likely solution state structures of $\mathrm{RMgX}(\mathrm{R}=\mathrm{Et}, i \mathrm{Pr}, n \mathrm{Bu}, n \mathrm{Hex}, n \mathrm{Oct}, n \mathrm{Dec} ; \mathrm{X}=\mathrm{Cl}, \mathrm{Br})$.

\begin{tabular}{lccccccccc}
\hline Aggregate & $\begin{array}{c}M W \\
{[\mathbf{g} / \mathbf{m o l}]}\end{array}$ & $\mathbf{C}$ & $\mathbf{H}$ & $\mathbf{O}$ & $\mathbf{M g}$ & $\mathbf{C l}$ & $\mathbf{B r}$ & $\begin{array}{c}M D_{\mathbf{W}} \\
{\left[\mathbf{g} /\left(\mathbf{m o l} \cdot \mathbf{m}^{3}\right)\right]}\end{array}$ & $\begin{array}{c}\Sigma V_{\mathbf{W}} \\
{\left[\mathbf{m}^{3}\right]}\end{array}$ \\
\hline$\left[\mathrm{EtMgCl}(\mathrm{THF})_{2}\right]$ & 233 & 10 & 21 & 2 & 1 & 1 & 0 & $5.88 \mathrm{E}+29$ & $3.96 \mathrm{E}-28$ \\
{$\left[\boldsymbol{i P r M g C l}(\mathrm{THF})_{2}\right]$} & 247 & 11 & 23 & 2 & 1 & 1 & 0 & $5.77 \mathrm{E}+29$ & $4.28 \mathrm{E}-28$ \\
{$\left[\boldsymbol{n B u M g C l}(\mathrm{THF})_{2}\right]$} & 261 & 12 & 25 & 2 & 1 & 1 & 0 & $5.68 \mathrm{E}+29$ & $4.60 \mathrm{E}-28$ \\
{$\left[\boldsymbol{n H e x M g C l}(\mathrm{THF})_{2}\right]$} & 289 & 14 & 29 & 2 & 1 & 1 & 0 & $5.52 \mathrm{E}+29$ & $5.23 \mathrm{E}-28$ \\
{$\left[\boldsymbol{n O c t M g C l}(\mathrm{THF})_{2}\right]$} & 317 & 16 & 33 & 2 & 1 & 1 & 0 & $5.41 \mathrm{E}+29$ & $5.87 \mathrm{E}-28$ \\
{$\left[\boldsymbol{n D e c M g C l}(\mathrm{THF})_{2}\right]$} & 345 & 18 & 37 & 2 & 1 & 1 & 0 & $5.31 \mathrm{E}+29$ & $6.50 \mathrm{E}-28$ \\
{$\left[\boldsymbol{n B u M g B r}(\mathrm{THF})_{2}\right]$} & 306 & 12 & 25 & 2 & 1 & 0 & 1 & $6.59 \mathrm{E}+29$ & $4.64 \mathrm{E}-28$ \\
\hline
\end{tabular}




\section{ECC-MW Estimations of $[n \mathrm{BuMgX} \cdot \mathrm{LiX}](\mathrm{X}=\mathrm{Cl}, \mathrm{Br})$}

\section{ECC-MW Estimation of $[n \mathrm{BuMgCl} \cdot \mathrm{LiCl}]$ in THF}

Table 5-48: ${ }^{1} \mathrm{H}-\mathrm{DOSY}-\mathrm{ECC}-M W$ estimation of $[n \mathrm{BuMgCl} \cdot \mathrm{LiCl}]$ in THF- $d_{8}(25 \mathrm{mM})$ at $25^{\circ} \mathrm{C}$. TPhN was used as internal reference with $\log \left(D_{\text {reffix }}\right)(\mathrm{TPhN})=-9.1054$. The accuracy of the $\operatorname{ECC}^{\mathrm{THF}}$ (DSE) is in the range of $M W_{\text {dif }}= \pm 8 \%$. Hypothetical aggregates are $\left[n \mathrm{BuMgCl}(\mathrm{THF})_{\mathrm{x}} \cdot \mathrm{LiCl}\right]$ with $\mathrm{x}=2-3$ and $[n \mathrm{BuMgCl}(\mathrm{THF}) \cdot \mathrm{LiCl}]_{2}$. For $\mathrm{ECC}^{\mathrm{THF}}$ parameters and changes compared to literature ${ }^{[109 \mathrm{a}]}$ see section 2.1.1.

\begin{tabular}{|c|c|c|c|c|}
\hline${ }^{1} \mathrm{H}$-DOSY & & & & $25^{\circ} \mathrm{C}$ \\
\hline & & Aggregate & $M W_{\text {calc }}[\mathrm{g} / \mathrm{mol}]$ & $M W_{\text {dif }}[\%]$ \\
\hline$D_{\mathbf{x}}\left[\mathrm{m}^{2} / \mathrm{s}\right]$ & $9.9193 \mathrm{E}-10$ & {$\left[n \mathrm{BuMgCl}(\mathrm{THF})_{2} \cdot \mathrm{LiCl}\right]$} & 303 & -16 \\
\hline $\log \left(D_{x}\right)$ & -9.0035 & {$\left[n \mathrm{BuMgCl}(\mathrm{THF})_{3} \cdot \mathrm{LiCl}\right]$} & 376 & 4 \\
\hline $\log \left(D_{\mathrm{x}, \text { norm }}\right)$ & -9.0293 & {$[n \mathrm{BuMgCl}(\mathrm{THF}) \cdot \mathrm{LiCl}]_{2}$} & 463 & 28 \\
\hline$D_{\text {ref }}(\mathrm{TPhN})\left[\mathrm{m}^{2} / \mathrm{s}\right]$ & $8.325 \mathrm{E}-10$ & & & \\
\hline $\log \left(D_{\text {ref }}\right)(\mathrm{TPhN})$ & -9.0796 & & & \\
\hline$M W_{\text {det }}[\mathrm{g} / \mathbf{m o l}]$ & 362 & & & \\
\hline$\Delta M W_{\text {det }}[\mathrm{g} / \mathrm{mol}](\%)$ & $\pm 20(6)$ & & & \\
\hline
\end{tabular}

\section{ECC-MW Estimation of $[n \mathrm{BuMgBr} \cdot \mathrm{LiBr}]$ in $\mathrm{THF}$}

Table 5-49: ${ }^{1} \mathrm{H}$-DOSY-ECC- $M W$ estimation of $[n \mathrm{BuMgBr} \cdot \mathrm{LiBr}]$ in THF- $d_{8}(25 \mathrm{mM})$ at $25^{\circ} \mathrm{C}$. TPhN was used as internal reference with $\log \left(D_{\text {reffix }}\right)(\mathrm{TPhN})=-9.1054$. The accuracy of the ECC ${ }^{\mathrm{THF}}\left(\mathrm{Br}_{2}\right)$ is in the range of $M W_{\text {dif }}= \pm 18 \%$. Hypothetical aggregates are $\left[n \mathrm{BuMgBr}(\mathrm{THF})_{\mathrm{x}} \cdot \mathrm{LiBr}\right]$ with $\mathrm{x}=2-3$ and $[n \mathrm{BuMgBr}(\mathrm{THF}) \cdot \mathrm{LiBr}]_{2}$. For ECC ${ }^{\mathrm{THF}}$ parameters see reference ${ }^{[124]}$.

\begin{tabular}{|c|c|c|c|c|}
\hline${ }^{1} \mathrm{H}-\mathrm{DOSY}$ & & & & $25^{\circ} \mathrm{C}$ \\
\hline & & Aggregate & $M W_{\text {calc }}[\mathrm{g} / \mathrm{mol}]$ & $M W_{\text {dif }}[\%]$ \\
\hline$D_{\mathbf{x}}\left[\mathrm{m}^{2} / \mathrm{s}\right]$ & $1.136 \mathrm{E}-09$ & {$\left[n \mathrm{BuMgBr}(\mathrm{THF})_{2} \cdot \mathrm{LiBr}\right]$} & 392 & -5 \\
\hline $\log \left(D_{\mathbf{x}}\right)$ & -8.9447 & {$\left[n \mathrm{BuMgBr}(\mathrm{THF})_{3} \cdot \mathrm{LiBr}\right]$} & 465 & 12 \\
\hline $\log \left(D_{\mathrm{x}, \text { norm }}\right)$ & -8.9816 & {$[n \mathrm{BuMgBr}(\mathrm{THF}) \cdot \mathrm{LiBr}]_{2}$} & 641 & 55 \\
\hline$D_{\text {ref }}(\mathrm{TPhN})\left[\mathrm{m}^{2} / \mathrm{s}\right]$ & $8.541 \mathrm{E}-10$ & & & \\
\hline $\log \left(D_{\text {ref }}\right)(\mathrm{TPhN})$ & -9.0685 & & & \\
\hline$M W_{\text {det }}[\mathrm{g} / \mathrm{mol}]$ & 413 & & & \\
\hline$\Delta M W_{\text {det }}[\mathrm{g} / \mathrm{mol}](\%)$ & $\pm 96(23)$ & & & \\
\hline
\end{tabular}

\section{Molar Van-Der-Waals Densities $\left(M D_{\mathrm{w}}\right)$}

Table 5-50: $M D_{\mathrm{W}}$ for most likely solution state structures of $[n \mathrm{BuMgX} \cdot \mathrm{LiX}](\mathrm{X}=\mathrm{Cl}, \mathrm{Br})$.

\begin{tabular}{lcccccccccc}
\hline Aggregate & $\begin{array}{c}M W \\
{[\mathrm{~g} / \mathbf{m o l}]}\end{array}$ & $\mathbf{C}$ & $\mathbf{H}$ & $\mathbf{O}$ & $\mathbf{L i}$ & $\mathbf{M g}$ & $\mathrm{Cl}$ & $\mathrm{Br}$ & $\begin{array}{c}M D_{\mathrm{W}} \\
{\left[\mathbf{g} /\left(\mathbf{m o l} \cdot \mathbf{m}^{3}\right)\right]}\end{array}$ & $\begin{array}{c}\Sigma V_{\mathbf{W}} \\
{\left[\mathbf{m}^{3}\right]}\end{array}$ \\
\hline$\left[\boldsymbol{n B u M g C l}(\mathrm{THF})_{3} \cdot \mathbf{L i C l}\right]$ & 376 & 16 & 33 & 3 & 1 & 1 & 2 & 0 & $5.79 \mathrm{E}+29$ & $6.49 \mathrm{E}-28$ \\
{$\left[n\right.$ BuMgBr$\left.(\mathrm{THF})_{3} \cdot \mathbf{L i B r}\right]$} & 465 & 16 & 33 & 3 & 1 & 1 & 0 & 2 & $7.61 \mathrm{E}+29$ & $6.58 \mathrm{E}-28$ \\
\hline
\end{tabular}




\section{ECC-MW Estimations of (IPrPh)X $\left(\mathrm{X}=\mathrm{Br}, \mathrm{I}, \mathrm{OTf}, \mathrm{BF}_{4}, \mathrm{PF}_{6}\right)^{\mathrm{a}}$}

\section{ECC-MW Estimation of (IPrPh)Br in Dichloromethane}

Table 5-51: ${ }^{1} \mathrm{H}$-DOSY-ECC- $M W$ estimation of (IPrPh) Br in $\mathrm{CD}_{2} \mathrm{Cl}_{2}$ at $25^{\circ} \mathrm{C}$. Adam was used as internal reference with $\log \left(D_{\text {ref,fix }}\right)($ Adam $)=-8.7035$. The accuracy of the $\mathrm{ECC}^{\mathrm{CD} 2 \mathrm{Cl} 2}(\mathrm{DSE})$ is in the range of $M W_{\mathrm{dif}}= \pm 14 \%$. Hypothetical aggregates are (IPrPh) ${ }^{+}$and $(\mathrm{IPrPh}) \mathrm{Br}$. For $\mathrm{ECC}^{\mathrm{CD} 2 \mathrm{Cl} 2}$-parameters see Figure 5-4.

\begin{tabular}{|c|c|c|c|c|}
\hline${ }^{1} \mathrm{H}-\mathrm{DOSY}$ & & & & $25^{\circ} \mathrm{C}$ \\
\hline & & Aggregate & $M W_{\text {calc }}[\mathrm{g} / \mathrm{mol}]$ & $M W_{\text {dif }}[\%]$ \\
\hline$D_{\mathbf{x}}\left[\mathrm{m}^{2} / \mathrm{s}\right]$ & $8.735 \mathrm{E}-10$ & $(\mathrm{IPrPh})^{+}$ & 466 & -21 \\
\hline $\log \left(D_{x}\right)$ & -9.0587 & $(\mathrm{IPrPh}) \mathrm{Br}$ & 546 & -8 \\
\hline $\log \left(D_{\mathrm{x}, \text { norm }}\right)$ & -9.0589 & & & \\
\hline$D_{\text {ref }}($ Adam $)\left[\mathrm{m}^{2} / \mathrm{s}\right]$ & $1.980 \mathrm{E}-09$ & & & \\
\hline $\log \left(D_{\text {ref }}\right)($ Adam $)$ & -8.7033 & & & \\
\hline$M W_{\operatorname{det}}[\mathrm{g} / \mathrm{mol}]$ & 590 & & & \\
\hline$\Delta M W_{\text {det }}[\mathrm{g} / \mathrm{mol}](\%)$ & $\pm 60(10)$ & & & \\
\hline
\end{tabular}

\section{ECC-MW Estimation of (IPrPh)I in Dichloromethane}

Table 5-52: ${ }^{1} \mathrm{H}$-DOSY-ECC- $M W$ estimation of (IPrPh)I in $\mathrm{CD}_{2} \mathrm{Cl}_{2}$ at $25^{\circ} \mathrm{C}$. Adam was used as internal reference with $\log \left(D_{\text {ref,fix }}\right)($ Adam $)=-8.7035$. The accuracy of the $\mathrm{ECC}^{\mathrm{CD} 2 \mathrm{Cl} 2}(\mathrm{DSE})$ is in the range of $M W_{\mathrm{dif}}= \pm 14 \%$. Hypothetical aggregates are $(\mathrm{IPrPh})^{+}$and $(\mathrm{IPrPh}) \mathrm{I}$. For $\mathrm{ECC}^{\mathrm{CD} 2 \mathrm{Cl} 2}$-parameters see Figure 5-4.

\begin{tabular}{|c|c|c|c|c|}
\hline${ }^{1} \mathrm{H}-\mathrm{DOSY}$ & & & & $25^{\circ} \mathrm{C}$ \\
\hline & & Aggregate & $M W_{\text {calc }}[\mathrm{g} / \mathrm{mol}]$ & $M W_{\text {dif }}[\%]$ \\
\hline$D_{\mathrm{x}}\left[\mathrm{m}^{2} / \mathrm{s}\right]$ & $9.610 \mathrm{E}-10$ & $(\mathrm{IPrPh})^{+}$ & 466 & -15 \\
\hline $\log \left(D_{x}\right)$ & -9.0173 & (IPrPh)I & 593 & 8 \\
\hline $\log \left(D_{\mathrm{x}, \mathrm{norm}}\right)$ & -9.0416 & & & \\
\hline$D_{\text {ref }}($ Adam $)\left[\mathrm{m}^{2} / \mathrm{s}\right]$ & $2.093 \mathrm{E}-09$ & & & \\
\hline $\log \left(D_{\text {ref }}\right)($ Adam $)$ & -8.6792 & & & \\
\hline$M W_{\text {det }}[\mathrm{g} / \mathrm{mol}]$ & 551 & & & \\
\hline$\Delta M W_{\text {det }}[\mathrm{g} / \mathrm{mol}](\%)$ & $\pm 55(10)$ & & & \\
\hline
\end{tabular}

\section{ECC-MW Estimation of (IPrPh)OTf in Dichloromethane}

Table 5-53: ${ }^{1} \mathrm{H}$-DOSY-ECC-MW estimation of (IPrPh)OTf in $\mathrm{CD}_{2} \mathrm{Cl}_{2}$ at $25^{\circ} \mathrm{C}$. Adam was used as internal reference with $\log \left(D_{\text {reffix }}\right)($ Adam $)=-8.7035$. The accuracy of the $\mathrm{ECC}^{\mathrm{CD} 2 \mathrm{Cl} 2}(\mathrm{DSE})$ is in the range of $M W_{\mathrm{dif}}= \pm 14 \%$. Hypothetical aggregates are $(\mathrm{IPrPh})^{+}$and $(\mathrm{IPrPh}) \mathrm{OTf}$. For $\mathrm{ECC}^{\mathrm{CD} 2 \mathrm{Cl} 2}$-parameters see Figure 5-4.

\begin{tabular}{|c|c|c|c|c|}
\hline${ }^{1} \mathrm{H}$-DOSY & & & & $25^{\circ} \mathrm{C}$ \\
\hline & & Aggregate & $M W_{\text {calc }}[\mathrm{g} / \mathrm{mol}]$ & $M W_{\text {dif }}[\%]$ \\
\hline$D_{\mathbf{x}}\left[\mathrm{m}^{2} / \mathrm{s}\right]$ & $8.894 \mathrm{E}-10$ & $(\mathrm{IPrPh})^{+}$ & 466 & -17 \\
\hline $\log \left(D_{x}\right)$ & -9.0509 & (IPrPh)OTf & 615 & 9 \\
\hline $\log \left(D_{\mathrm{x}, \text { norm }}\right)$ & -9.0474 & & & \\
\hline$D_{\text {ref }}($ Adam $)\left[\mathrm{m}^{2} / \mathrm{s}\right]$ & $1.964 \mathrm{E}-09$ & & & \\
\hline $\log \left(D_{\text {ref }}\right)($ Adam $)$ & -8.7070 & & & \\
\hline$M W_{\text {det }}[\mathrm{g} / \mathrm{mol}]$ & 564 & & & \\
\hline$\Delta M W_{\operatorname{det}}[\mathrm{g} / \mathrm{mol}](\%)$ & $\pm 57(10)$ & & & \\
\hline
\end{tabular}

${ }^{a}$ For further information, see section 2.3.2.1. 


\section{ECC-MW Estimation of (IPrPh)BF 4 in Dichloromethane}

Table 5-54: ${ }^{1} \mathrm{H}$-DOSY-ECC- $M W$ estimation of $(\mathrm{IPrPh}) \mathrm{BF}_{4}$ in $\mathrm{CD}_{2} \mathrm{Cl}_{2}$ at $25^{\circ} \mathrm{C}$. Adam was used as internal reference with $\log \left(D_{\text {ref, fix }}\right)($ Adam $)=-8.7035$. The accuracy of the $\mathrm{ECC}^{\mathrm{CD} 2 \mathrm{Cl} 2}(\mathrm{DSE})$ is in the range of $M W_{\mathrm{dif}}= \pm 14 \%$. Hypothetical aggregates are $(\mathrm{IPrPh})^{+}$and $(\mathrm{IPrPh}) \mathrm{BF}_{4}$. For $\mathrm{ECC}^{\mathrm{CD} 2 \mathrm{Cl} 2}$-parameters see Figure 5-4.

\begin{tabular}{|c|c|c|c|c|}
\hline${ }^{1} \mathrm{H}-\mathrm{DOSY}$ & & & & $25^{\circ} \mathrm{C}$ \\
\hline & & Aggregate & $M W_{\text {calc }}[\mathrm{g} / \mathrm{mol}]$ & $M W_{\text {dif }}[\%]$ \\
\hline$D_{\mathbf{x}}\left[\mathrm{m}^{2} / \mathrm{s}\right]$ & $8.506 \mathrm{E}-10$ & $(\mathrm{IPrPh})^{+}$ & 466 & -19 \\
\hline $\log \left(D_{\mathbf{x}}\right)$ & -9.0703 & $(\mathrm{IPrPh}) \mathrm{BF}_{4}$ & 553 & -4 \\
\hline $\log \left(D_{\mathrm{x}, \text { norm }}\right)$ & -9.0515 & & & \\
\hline$D_{\text {ref }}\left(\right.$ Adam) $\left[\mathrm{m}^{2} / \mathrm{s}\right]$ & $1.896 \mathrm{E}-09$ & & & \\
\hline $\log \left(D_{\text {ref }}\right)($ Adam $)$ & -8.7223 & & & \\
\hline$M W_{\text {det }}[\mathrm{g} / \mathrm{mol}]$ & 573 & & & \\
\hline$\Delta M W_{\text {det }}[\mathrm{g} / \mathrm{mol}](\%)$ & $\pm 58(10)$ & & & \\
\hline
\end{tabular}

\section{ECC-MW Estimation of (IPrPh)PF 6 in Dichloromethane}

Table 5-55: ${ }^{1} \mathrm{H}$-DOSY-ECC- $M W$ estimation of ( $\left.\mathrm{IPrPh}\right) \mathrm{PF}_{6}$ in $\mathrm{CD}_{2} \mathrm{Cl}_{2}$ at $25^{\circ} \mathrm{C}$. Adam was used as internal reference with $\log \left(D_{\text {reffix }}\right)($ Adam $)=-8.7035$. The accuracy of the $\mathrm{ECC}^{\mathrm{CD} 2 \mathrm{Cl} 2}(\mathrm{DSE})$ is in the range of $M W_{\mathrm{dif}}= \pm 14 \%$. Hypothetical aggregates are $(\mathrm{IPrPh})^{+}$and $(\mathrm{IPrPh}) \mathrm{PF}_{6}$. For $\mathrm{ECC}^{\mathrm{CD} 2 \mathrm{Cl} 2}$-parameters see Figure 5-4.

\begin{tabular}{|c|c|c|c|c|}
\hline${ }^{1} \mathrm{H}-\mathrm{DOSY}$ & & & & $25^{\circ} \mathrm{C}$ \\
\hline & & Aggregate & $M W_{\text {calc }}[\mathrm{g} / \mathrm{mol}]$ & $M W_{\text {dif }}[\%]$ \\
\hline$D_{\mathrm{x}}\left[\mathrm{m}^{2} / \mathrm{s}\right]$ & $8.510 \mathrm{E}-10$ & $(\mathrm{IPrPh})^{+}$ & 466 & -18 \\
\hline $\log \left(D_{\mathbf{x}}\right)$ & -9.0701 & $(\mathrm{IPrPh}) \mathrm{PF}_{6}$ & 611 & 8 \\
\hline $\log \left(D_{\mathrm{x}, \text { norm }}\right)$ & -9.0480 & & & \\
\hline$D_{\text {ref }}\left(\right.$ Adam) $\left[\mathrm{m}^{2} / \mathrm{s}\right]$ & $1.881 \mathrm{E}-09$ & & & \\
\hline $\log \left(D_{\text {ref }}\right)($ Adam $)$ & -8.7256 & & & \\
\hline$M W_{\operatorname{det}}[\mathrm{g} / \mathrm{mol}]$ & 565 & & & \\
\hline$\Delta M W_{\text {det }}[\mathrm{g} / \mathrm{mol}](\%)$ & $\pm 57(10)$ & & & \\
\hline
\end{tabular}

\section{Molar Van-Der-Waals Densities $\left(M D_{\mathrm{w}}\right)$}

Table 5-56: $M D_{\mathrm{w}}$ for all hypothetical solution state structures of Table 5-51 to Table 5-55.

\begin{tabular}{lcccccccccccc}
\hline Aggregate & $\begin{array}{c}\boldsymbol{M W} \\
{[\mathbf{g} / \mathbf{m o l}]}\end{array}$ & $\mathbf{C}$ & $\mathbf{H}$ & $\mathbf{N}$ & $\mathbf{O}$ & $\mathbf{F}$ & $\mathbf{B}$ & $\mathbf{B r}$ & $\mathbf{I}$ & $\mathbf{S}$ & $\mathbf{P}$ & $\begin{array}{c}\boldsymbol{M D}_{\mathbf{W}} \\
{\left[\mathbf{g} /\left(\mathbf{m o l} \cdot \mathbf{~ m}^{3}\right)\right]}\end{array}$ \\
\hline $\mathbf{( I P r P h}^{+}$ & 466 & 33 & 41 & 2 & 0 & 0 & 0 & 0 & 0 & 0 & 0 & $4.96 \mathrm{E}+29$ \\
$(\mathbf{I P r P h}) \mathbf{B r}$ & 546 & 33 & 41 & 2 & 0 & 0 & 0 & 1 & 0 & 0 & 0 & $5.65 \mathrm{E}+29$ \\
$(\mathbf{I P r P h}) \mathbf{I}$ & 593 & 33 & 41 & 2 & 0 & 0 & 0 & 0 & 1 & 0 & 0 & $6.10 \mathrm{E}+29$ \\
$(\mathbf{I P r P h}) \mathbf{O T f}$ & 615 & 34 & 41 & 2 & 3 & 3 & 0 & 0 & 0 & 1 & 0 & $5.76 \mathrm{E}+29$ \\
$(\mathbf{I P r P h}) \mathbf{B F}_{4}$ & 553 & 33 & 41 & 2 & 0 & 4 & 1 & 0 & 0 & 0 & 0 & $5.41 \mathrm{E}+29$ \\
$(\mathbf{I P r P h}) \mathbf{P F}_{6}$ & 611 & 33 & 41 & 2 & 0 & 6 & 0 & 0 & 0 & 0 & 1 & $5.85 \mathrm{E}+29$ \\
\hline
\end{tabular}




\section{ECC-MW Estimations of $\left(4,6-t \mathrm{Bu}-\mathrm{NCOC}_{6} \mathrm{H}_{2}\right)_{2} \mathrm{CH}_{2}$ and $\left[\mathrm{M}\left(4,6-\mathrm{tBu}-\mathrm{NCOC}{ }_{6} \mathrm{H}_{2}\right)_{2} \mathrm{CH}\right](\mathrm{M}=\mathrm{Li}, \mathrm{K}, \mathrm{Mg})^{\mathrm{a}}$}

\section{ECC-MW Estimation of the $\left(4,6-t B u-\mathrm{NCOC}_{6} \mathrm{H}_{2}\right)_{2} \mathrm{CH}_{2}$ Ligand in Benzene}

Table 5-57: ${ }^{1} \mathrm{H}$-DOSY-ECC- $M W$ estimation of the $\left(4,6-t \mathrm{Bu}-\mathrm{NCOC}_{6} \mathrm{H}_{2}\right)_{2} \mathrm{CH}_{2}$ ligand in $\mathrm{C}_{6} \mathrm{D}_{6}$ at $25^{\circ} \mathrm{C}$. TMB was used as internal reference with $\log \left(D_{\text {ref,fix }}\right)(\mathrm{TMB})=-8.7771$. The accuracy of the $\mathrm{ECC}^{\mathrm{C} 6 \mathrm{D} 6}(\mathrm{ED})$ is in the range of $M W_{\mathrm{dif}}= \pm 6 \%$. Hypothetical aggregate is $\left(4,6-t \mathrm{Bu}-\mathrm{NCOC}_{6} \mathrm{H}_{2}\right)_{2} \mathrm{CH}_{2}$. For ECC ${ }^{\mathrm{C} 6 \mathrm{D} 6}$-parameters see Figure 5-4.

\begin{tabular}{|c|c|c|c|c|}
\hline${ }^{1} \mathrm{H}-\mathrm{DOSY}$ & & & & $25^{\circ} \mathrm{C}$ \\
\hline & & Aggregate & $M W_{\text {calc }}[\mathrm{g} / \mathrm{mol}]$ & $M W_{\text {dif }}[\%]$ \\
\hline$D_{\mathbf{x}}\left[\mathrm{m}^{2} / \mathrm{s}\right]$ & $6.851 \mathrm{E}-10$ & $\left(4,6-t \mathrm{Bu}-\mathrm{NCOC}_{6} \mathrm{H}_{2}\right)_{2} \mathrm{CH}_{2}$ & 475 & -2 \\
\hline $\log \left(D_{x}\right)$ & -9.1642 & & & \\
\hline $\log \left(D_{\mathrm{x}, \mathrm{norm}}\right)$ & -9.1720 & & & \\
\hline$D_{\text {ref }}(\mathrm{TMB})\left[\mathrm{m}^{2} / \mathrm{s}\right]$ & $1.701 \mathrm{E}-10$ & & & \\
\hline $\log \left(D_{\text {ref }}\right)($ TMB $)$ & -8.7693 & & & \\
\hline$M W_{\text {det }}[\mathrm{g} / \mathrm{mol}]$ & 483 & & & \\
\hline$\Delta M W_{\text {det }}[\mathrm{g} / \mathrm{mol}](\%)$ & $\pm 61(13)$ & & & \\
\hline
\end{tabular}

\section{ECC-MW Estimation of $\left[\mathrm{Li}\left(4,6-\mathrm{tBu}-\mathrm{NCOC}_{6} \mathrm{H}_{2}\right){ }_{2} \mathrm{CH}\right]$ in Benzene}

Table 5-58: ${ }^{1} \mathrm{H}-\mathrm{DOSY}-\mathrm{ECC}-\mathrm{MW}$ estimation of $\left[\mathrm{Li}\left(4,6-t \mathrm{Bu}-\mathrm{NCOC}_{6} \mathrm{H}_{2}\right)_{2} \mathrm{CH}\right]$ in $\mathrm{C}_{6} \mathrm{D}_{6}$ at $25^{\circ} \mathrm{C}$. TMB was used as internal reference with $\log \left(D_{\text {ref,fix }}\right)(\mathrm{TMB})=-8.7771$. The accuracy of the ECC C6D6 (DSE) is in the range of $M W_{\mathrm{dif}}= \pm 8 \%$. Hypothetical aggregates are $\left[\mathrm{Li}\left(4,6-t \mathrm{Bu}-\mathrm{NCOC}_{6} \mathrm{H}_{2}\right)_{2} \mathrm{CH}(\mathrm{THF})_{\mathrm{x}}\right]$ with $\mathrm{x}=0-2$. For $\mathrm{ECC}^{\mathrm{C} 6 \mathrm{D} 6}$-parameters see Figure 5-4. Coordinated THF originates from synthesis.

\begin{tabular}{lrlll}
\hline${ }^{1} \mathbf{H}-\mathrm{DOSY}$ & & & & $\mathbf{2 5}^{\circ} \mathrm{C}$ \\
\hline & & Aggregate & $\boldsymbol{M} \boldsymbol{W}_{\text {calc }}[\mathbf{g} / \mathbf{m o l}]$ & $\boldsymbol{M} \boldsymbol{W}_{\text {dif }}[\%]$ \\
$\boldsymbol{D}_{\mathbf{x}}\left[\mathbf{m}^{2} / \mathbf{s}\right]$ & $6.357 \mathrm{E}-10$ & {$\left[\mathrm{Li}\left(4,6-t \mathrm{Bu}-\mathrm{NCOC} \mathrm{H}_{2}\right)_{2} \mathrm{CH}\right]$} & 481 & -16 \\
$\log \left(\boldsymbol{D}_{\mathbf{x}}\right)$ & -9.1967 & {$\left[\mathrm{Li}\left(4,6-t \mathrm{Bu}-\mathrm{NCOC}_{6} \mathrm{H}_{2}\right)_{2} \mathrm{CH}(\mathrm{THF})\right]$} & 553 & -4 \\
$\log \left(\boldsymbol{D}_{\mathbf{x}, \text { norm }}\right)$ & -9.1863 & {$\left[\mathrm{Li}\left(4,6-t \mathrm{Bu}-\mathrm{NCOC}_{6} \mathrm{H}_{2}\right)_{2} \mathrm{CH}(\mathrm{THF})_{2}\right]$} & 625 & 9 \\
$\boldsymbol{D}_{\text {ref }}(\mathrm{TMB})\left[\mathbf{m}^{2} / \mathbf{s}\right]$ & $1.631 \mathrm{E}-10$ & & & \\
$\log \left(\boldsymbol{D}_{\text {ref }}\right)(\mathrm{TMB})$ & -8.7875 & & & \\
$\boldsymbol{M} \boldsymbol{W}_{\text {det }}[\mathbf{g} / \mathbf{m o l}]$ & 575 & & \\
$\Delta \boldsymbol{M} \boldsymbol{W}_{\text {det }}[\mathbf{g} / \mathbf{m o l}](\%)$ & $\pm 32(6)$ & & \\
\end{tabular}

\section{ECC-MW Estimation of $\left[K\left(4,6-t B u-N \mathrm{NOC}_{6} \mathrm{H}_{2}\right)_{2} \mathrm{CH}\right]$ in Benzene}

Table 5-59: ${ }^{1} \mathrm{H}-\mathrm{DOSY}-\mathrm{ECC}-\mathrm{MW}$ estimation of $\left[\mathrm{K}\left(4,6-t \mathrm{Bu}-\mathrm{NCOC}_{6} \mathrm{H}_{2}\right)_{2} \mathrm{CH}\right]$ in $\mathrm{C}_{6} \mathrm{D}_{6}$ at $25^{\circ} \mathrm{C}$. TMB was used as internal reference with $\log \left(D_{\text {reffix }}\right)(\mathrm{TMB})=-8.7771$. The accuracy of the $\mathrm{ECC}^{\mathrm{C} 6 \mathrm{D} 6}$ (DSE) is in the range of $M W_{\mathrm{dif}}= \pm 8 \%$. Hypothetical aggregates are $\left[\mathrm{K}\left(4,6-t \mathrm{Bu}-\mathrm{NCOC}_{6} \mathrm{H}_{2}\right)_{2} \mathrm{CH}(\mathrm{THF})_{\mathrm{x}}\right]$ with $\mathrm{x}=0-2$. For ECC ${ }^{\mathrm{C} 6 \mathrm{D} 6}$-parameters see Figure 5-4. Coordinated THF originates from synthesis.

\begin{tabular}{|c|c|c|c|c|}
\hline${ }^{1} \mathrm{H}-\mathrm{DOSY}$ & & & & $25^{\circ} \mathrm{C}$ \\
\hline & & Aggregate & $M W_{\text {calc }}[\mathrm{g} / \mathrm{mol}]$ & $M W_{\text {dif }}[\%]$ \\
\hline$D_{\mathbf{x}}\left[\mathrm{m}^{2} / \mathrm{s}\right]$ & $7.050 \mathrm{E}-10$ & {$\left[\mathrm{~K}\left(4,6-\mathrm{tBu}-\mathrm{NCOC}_{6} \mathrm{H}_{2}\right)_{2} \mathrm{CH}\right]$} & 513 & -8 \\
\hline $\log \left(D_{\mathbf{x}}\right)$ & -9.1518 & {$\left[\mathrm{~K}\left(4,6-t \mathrm{Bu}-\mathrm{NCOC}_{6} \mathrm{H}_{2}\right)_{2} \mathrm{CH}(\mathrm{THF})\right]$} & 585 & 5 \\
\hline $\log \left(D_{\mathrm{x}, \text { norm }}\right)$ & -9.1774 & {$\left[\mathrm{~K}\left(4,6-t \mathrm{Bu}-\mathrm{NCOC}_{6} \mathrm{H}_{2}\right)_{2} \mathrm{CH}(\mathrm{THF})_{2}\right]$} & 657 & 18 \\
\hline$D_{\text {ref }}(\mathrm{TMB})\left[\mathrm{m}^{2} / \mathrm{s}\right]$ & $1.772 \mathrm{E}-10$ & & & \\
\hline $\log \left(D_{\text {ref }}\right)($ TMB $)$ & -8.7515 & & & \\
\hline$M W_{\text {det }}[\mathrm{g} / \mathrm{mol}]$ & 556 & & & \\
\hline$\Delta M W_{\text {det }}[\mathrm{g} / \mathrm{mol}](\%)$ & $\pm 31(6)$ & & & \\
\hline
\end{tabular}

a As this is an ongoing investigation, synthetic and other structural information is not given. For further information, see section 2.3.2.3. 


\section{ECC-MW Estimation of $\left[\mathrm{MgCl}_{2}\left(4,6-\mathrm{tBu}-\mathrm{NCOC}_{6} \mathrm{H}_{2}\right)_{2} \mathrm{CH}\right]$ in Benzene}

Table 5-60: ${ }^{1} \mathrm{H}$-DOSY-ECC- $M W$ estimation of $\left[\mathrm{MgCl}_{2}\left(4,6-t \mathrm{Bu}-\mathrm{NCOC}_{6} \mathrm{H}_{2}\right)_{2} \mathrm{CH}\right]$ in $\mathrm{C}_{6} \mathrm{D}_{6}$ at $25^{\circ} \mathrm{C}$. TMB was used as internal reference with $\log \left(D_{\text {ref,fix }}\right)(\mathrm{TMB})=-8.7771$. The accuracy of the $\mathrm{ECC}^{\mathrm{C} 6 \mathrm{D} 6}$ (DSE) is in the range of $M W_{\mathrm{dif}}= \pm 8 \%$. Hypothetical aggregates are $\left[\mathrm{MgCl}_{2-\mathrm{x}}\left(4,6-t \mathrm{Bu}-\mathrm{NCOC}_{6} \mathrm{H}_{2}\right)_{2} \mathrm{CH}(\mathrm{THF})_{\mathrm{x}}\right]$ with $\mathrm{x}=0-2$. For $\mathrm{ECC}^{\mathrm{C} 6 \mathrm{D} 6}$-parameters see Figure 54. Coordinated THF originates from synthesis.

\begin{tabular}{lrlll}
\hline${ }^{1} \mathrm{H}-\mathrm{DOSY}$ & & & & $\mathbf{2 5}^{\circ} \mathrm{C}$ \\
\hline & & Aggregate & $\boldsymbol{M} \boldsymbol{W}_{\text {calc }}[\mathbf{g} / \mathbf{m o l}]$ & $\boldsymbol{M} \boldsymbol{W}_{\text {dif }}[\%]$ \\
$\boldsymbol{D}_{\mathbf{x}}\left[\mathbf{m}^{2} / \mathbf{s}\right]$ & $5.845 \mathrm{E}-10$ & {$\left[\mathrm{MgCl}_{2}\left(4,6-t \mathrm{Bu}-\mathrm{NCOC}_{6} \mathrm{H}_{2}\right)_{2} \mathrm{CH}\right]$} & 569 & -7 \\
$\log \left(\boldsymbol{D}_{\mathbf{x}}\right)$ & -9.2332 & {$\left[\mathrm{MgCl}\left(4,6-t \mathrm{Bu}-\mathrm{NCOC}_{6} \mathrm{H}_{2}\right)_{2} \mathrm{CH}(\mathrm{THF})\right]$} & 606 & -1 \\
$\log \left(\boldsymbol{D}_{\mathbf{x}, \text { norm }}\right)$ & -9.2020 & {$\left[\mathrm{Mg}\left(4,6-t \mathrm{Bu}-\mathrm{NCOC}_{6} \mathrm{H}_{2}\right)_{2} \mathrm{CH}(\mathrm{THF})_{2}\right]$} & 642 & 5 \\
$\boldsymbol{D}_{\text {ref }}(\mathrm{TMB})\left[\mathbf{m}^{2} / \mathbf{s}\right]$ & $1.555 \mathrm{E}-09$ & & \\
$\log \left(\boldsymbol{D}_{\text {ref }}\right)(\mathrm{TMB})$ & -8.8083 & & \\
$\boldsymbol{M} \boldsymbol{W}_{\text {det }}[\mathbf{g} / \mathbf{m o l}]$ & 609 & & \\
$\boldsymbol{M} \boldsymbol{W}_{\text {det }}[\mathbf{g} / \mathbf{m o l}](\%)$ & $\pm 34(6)$ & & \\
\hline
\end{tabular}

\section{ECC-MW Estimation of $\left[\mathrm{MgBr}_{2}\left(4,6-\mathrm{tBu}-\mathrm{NCOC}_{6} \mathrm{H}_{2}\right)_{2} \mathrm{CH}\right]$ in Benzene}

Table 5-61: ${ }^{1} \mathrm{H}-\mathrm{DOSY}-\mathrm{ECC}-\mathrm{MW}$ estimation of $\left[\mathrm{MgBr}_{2}\left(4,6-t \mathrm{Bu}-\mathrm{NCOC}_{6} \mathrm{H}_{2}\right)_{2} \mathrm{CH}\right]$ in $\mathrm{C}_{6} \mathrm{D}_{6}$ at $25{ }^{\circ} \mathrm{C}$. TMB was used as internal reference with $\log \left(D_{\text {ref,fix }}\right)(\mathrm{TMB})=-8.7771$. The accuracy of the $\mathrm{ECC}^{\mathrm{C} 6 \mathrm{D} 6}$ (DSE) is in the range of $M W_{\text {dif }}= \pm 8 \%$. Hypothetical aggregates are $\left[\mathrm{MgBr}_{2}-\mathrm{x}\left(4,6-t \mathrm{Bu}-\mathrm{NCOC}_{6} \mathrm{H}_{2}\right)_{2} \mathrm{CH}(\mathrm{THF})_{\mathrm{x}}\right]$ with $\mathrm{x}=0-2$. For ECC ${ }^{\mathrm{C} 6 \mathrm{D} 6}$-parameters see Figure 54. Coordinated THF originates from synthesis.

\begin{tabular}{lrlll}
\hline${ }^{1} \mathrm{H}-\mathrm{DOSY}$ & & & $\mathbf{2 5}^{\circ} \mathrm{C}$ \\
\hline & & Aggregate & $\boldsymbol{M} \boldsymbol{W}_{\text {calc }}[\mathbf{g} / \mathbf{m o l}]$ & $\boldsymbol{M} \boldsymbol{W}_{\text {dif }}[\%]$ \\
$\boldsymbol{D}_{\mathbf{x}}\left[\mathbf{m}^{2} / \mathbf{s}\right]$ & $5.476 \mathrm{E}-10$ & {$\left[\mathrm{MgBr}\left(4,6-t \mathrm{Bu}-\mathrm{NCOC}_{6} \mathrm{H}_{2}\right)_{2} \mathrm{CH}\right]$} & 658 & 4 \\
$\log \left(\boldsymbol{D}_{\mathbf{x}}\right)$ & -9.2616 & {$\left[\mathrm{MgBr}\left(4,6-t \mathrm{Bu}-\mathrm{NCOC}_{6} \mathrm{H}_{2}\right)_{2} \mathrm{CH}(\mathrm{THF})\right]$} & 650 & 3 \\
$\log \left(\boldsymbol{D}_{\mathbf{x}, \mathbf{n o r m}}\right)$ & -9.2127 & {$\left[\mathrm{Mg}\left(4,6-t \mathrm{Bu}-\mathrm{NCOC}_{6} \mathrm{H}_{2}\right)_{2} \mathrm{CH}(\mathrm{THF})_{2}\right]$} & 642 & 1 \\
$\boldsymbol{D}_{\text {ref }}(\mathbf{T M B})\left[\mathbf{m}^{2} / \mathbf{s}\right]$ & $1.493 \mathrm{E}-09$ & & \\
$\log \left(\boldsymbol{D}_{\text {ref }}\right)(\mathrm{TMB})$ & -8.8259 & & \\
$\boldsymbol{M} \boldsymbol{W}_{\text {det }}[\mathbf{g} / \mathbf{m o l}]$ & 634 & & \\
$\Delta \boldsymbol{M} \boldsymbol{W}_{\text {det }}[\mathbf{g} / \mathbf{m o l}](\%)$ & $\pm 36(6)$ & & \\
\hline
\end{tabular}

\section{ECC-MW Estimation of $\left[\mathrm{Mg}\left(\left(4,6-\mathrm{tBu}-\mathrm{NCOC}_{6} \mathrm{H}_{2}\right)_{2} \mathrm{CH}\right)_{2}\right]$ in Benzene}

Table 5-62: ${ }^{1} \mathrm{H}$-DOSY-ECC- $M W$ estimation of $\left[\mathrm{Mg}\left(\left(4,6-t \mathrm{Bu}-\mathrm{NCOC}_{6} \mathrm{H}_{2}\right)_{2} \mathrm{CH}\right)_{2}\right]$ in $\mathrm{C}_{6} \mathrm{D}_{6}$ at $25^{\circ} \mathrm{C}$. TMB was used as internal reference with $\log \left(D_{\text {reffix }}\right)(\mathrm{TMB})=-8.7771$. The accuracy of the ECC ${ }^{\mathrm{C} 6 \mathrm{D} 6}$ (merged) is in the range of $M W_{\text {dif }}= \pm 19 \%$. Hypothetical aggregate is $\left[\mathrm{Mg}\left(\left(4,6-t \mathrm{Bu}-\mathrm{NCOC}_{6} \mathrm{H}_{2}\right)_{2} \mathrm{CH}\right)_{2}\right]$. For ECC ${ }^{\mathrm{C} 6 \mathrm{D} 6}$-parameters see Figure 5-4.

\begin{tabular}{|c|c|c|c|c|}
\hline${ }^{1} \mathrm{H}-\mathrm{DOSY}$ & & & & $25^{\circ} \mathrm{C}$ \\
\hline & & Aggregate & $M W_{\text {calc }}[\mathrm{g} / \mathrm{mol}]$ & $M W_{\text {dif }}[\%]$ \\
\hline$D_{\mathrm{x}}\left[\mathrm{m}^{2} / \mathrm{s}\right]$ & $4.548 \mathrm{E}-10$ & {$\left[\mathrm{Mg}\left(\left(4,6-t \mathrm{Bu}-\mathrm{NCOC} \mathrm{H}_{2}\right)_{2} \mathrm{CH}\right)_{2}\right]$} & 972 & -2 \\
\hline $\log \left(D_{x}\right)$ & -9.3422 & & & \\
\hline $\log \left(D_{\mathbf{x}, \text { norm }}\right)$ & -9.2942 & & & \\
\hline$D_{\text {ref }}(\mathrm{TMB})\left[\mathrm{m}^{2} / \mathrm{s}\right]$ & $1.496 \mathrm{E}-09$ & & & \\
\hline $\log \left(D_{\text {ref }}\right)($ TMB $)$ & -8.8251 & & & \\
\hline$M W_{\operatorname{det}}[\mathrm{g} / \mathrm{mol}]$ & 993 & & & \\
\hline$\Delta M W_{\text {det }}[\mathrm{g} / \mathrm{mol}](\%)$ & $\pm 90(9)$ & & & \\
\hline
\end{tabular}




\section{Molar Van-Der-Waals Densities $\left(M D_{w}\right)$}

Table 5-63: $M D_{\mathrm{W}}$ for all hypothetical solution state structures of Table 5-57 to Table 5-62.

\begin{tabular}{|c|c|c|c|c|c|c|c|c|c|c|c|}
\hline Aggregate & $\begin{array}{c}M W \\
{[\mathrm{~g} / \mathrm{mol}]} \\
\end{array}$ & $\mathrm{C}$ & $\mathbf{H}$ & $\mathbf{N}$ & $\mathbf{O}$ & $\mathbf{L i}$ & $\mathbf{K}$ & $\mathrm{Mg}$ & $\mathrm{Cl}$ & $\mathrm{Br}$ & $\begin{array}{c}M D_{\mathrm{W}} \\
{\left[\mathrm{g} /\left(\mathbf{m o l} \cdot \mathbf{m}^{3}\right)\right]}\end{array}$ \\
\hline$\left(4,6-t \mathrm{Bu}-\mathrm{NCOC}{ }_{6} \mathrm{H}_{2}\right)_{2} \mathrm{CH}_{2}$ & 475 & 31 & 42 & 2 & 2 & 0 & 0 & 0 & 0 & 0 & $5.09 \mathrm{E}+29$ \\
\hline$\left[\mathrm{Li}\left(4,6-t \mathrm{Bu}-\mathrm{NCOC}_{6} \mathrm{H}_{2}\right)_{2} \mathrm{CH}\right]$ & 481 & 31 & 41 & 2 & 2 & 1 & 0 & 0 & 0 & 0 & $5.05 \mathrm{E}+29$ \\
\hline$\left[\mathrm{Li}\left(4,6-t \mathrm{Bu}-\mathrm{NCOC}{ }_{6} \mathrm{H}_{2}\right)_{2} \mathrm{CH}(\mathrm{THF})\right]$ & 553 & 35 & 49 & 2 & 3 & 1 & 0 & 0 & 0 & 0 & $5.05 \mathrm{E}+29$ \\
\hline$\left[\mathrm{Li}\left(4,6-t \mathrm{Bu}-\mathrm{NCOC}{ }_{6} \mathrm{H}_{2}\right)_{2} \mathrm{CH}(\mathrm{THF})_{2}\right]$ & 625 & 39 & 57 & 2 & 4 & 1 & 0 & 0 & 0 & 0 & $5.06 \mathrm{E}+29$ \\
\hline$\left[\mathrm{K}\left(4,6-t \mathrm{Bu}-\mathrm{NCOC}{ }_{6} \mathrm{H}_{2}\right)_{2} \mathrm{CH}\right]$ & 513 & 31 & 41 & 2 & 2 & 0 & 1 & 0 & 0 & 0 & $5.06 \mathrm{E}+29$ \\
\hline$\left[\mathrm{K}\left(4,6-t \mathrm{Bu}-\mathrm{NCOC}{ }_{6} \mathrm{H}_{2}\right)_{2} \mathrm{CH}(\mathrm{THF})\right]$ & 585 & 35 & 49 & 2 & 3 & 0 & 1 & 0 & 0 & 0 & $5.06 \mathrm{E}+29$ \\
\hline$\left[\mathrm{K}\left(4,6-t \mathrm{Bu}-\mathrm{NCOC} \mathrm{CH}_{2}\right)_{2} \mathrm{CH}(\mathrm{THF})_{2}\right]$ & 657 & 39 & 57 & 2 & 4 & 0 & 1 & 0 & 0 & 0 & $5.06 \mathrm{E}+29$ \\
\hline$\left[\mathrm{MgCl}_{2}\left(4,6-t \mathrm{Bu}-\mathrm{NCOC}{ }_{6} \mathrm{H}_{2}\right)_{2} \mathrm{CH}\right]$ & 569 & 31 & 41 & 2 & 2 & 0 & 0 & 1 & 2 & 0 & $5.72 \mathrm{E}+29$ \\
\hline$\left[\mathrm{MgCl}\left(4,6-t \mathrm{Bu}-\mathrm{NCOC} 6 \mathrm{H}_{2}\right)_{2} \mathrm{CH}(\mathrm{THF})\right]$ & 606 & 35 & 49 & 2 & 3 & 0 & 0 & 1 & 1 & 0 & $5.44 \mathrm{E}+29$ \\
\hline$\left[\mathrm{MgBr}_{2}\left(4,6-t \mathrm{Bu}-\mathrm{NCOC} \mathrm{H}_{2}\right)_{2} \mathrm{CH}\right]$ & 658 & 31 & 41 & 2 & 2 & 0 & 0 & 1 & 0 & 2 & $6.57 \mathrm{E}+29$ \\
\hline$\left[\mathrm{MgBr}\left(4,6-t \mathrm{Bu}-\mathrm{NCOC}{ }_{6} \mathrm{H}_{2}\right)_{2} \mathrm{CH}(\mathrm{THF})\right]$ & 650 & 35 & 49 & 2 & 3 & 0 & 0 & 1 & 0 & 1 & $5.82 \mathrm{E}+29$ \\
\hline$\left[\mathrm{Mg}\left(4,6-t \mathrm{Bu}-\mathrm{NCOC}{ }_{6} \mathrm{H}_{2}\right)_{2} \mathrm{CH}(\mathrm{THF})_{2}\right]$ & 642 & 39 & 57 & 2 & 4 & 0 & 0 & 1 & 0 & 0 & $5.21 \mathrm{E}+29$ \\
\hline$\left[\mathrm{Mg}\left(\left(4,6-t \mathrm{Bu}-\mathrm{NCOC} \mathrm{H}_{2}\right)_{2} \mathrm{CH}\right)_{2}\right]$ & 972 & 62 & 82 & 4 & 4 & 0 & 0 & 1 & 0 & 0 & $5.18 \mathrm{E}+29$ \\
\hline
\end{tabular}




\section{NMR Spectra}

\section{${ }^{1} \mathrm{H}$-DOSY NMR Spectrum of CpLi in Ammonia}

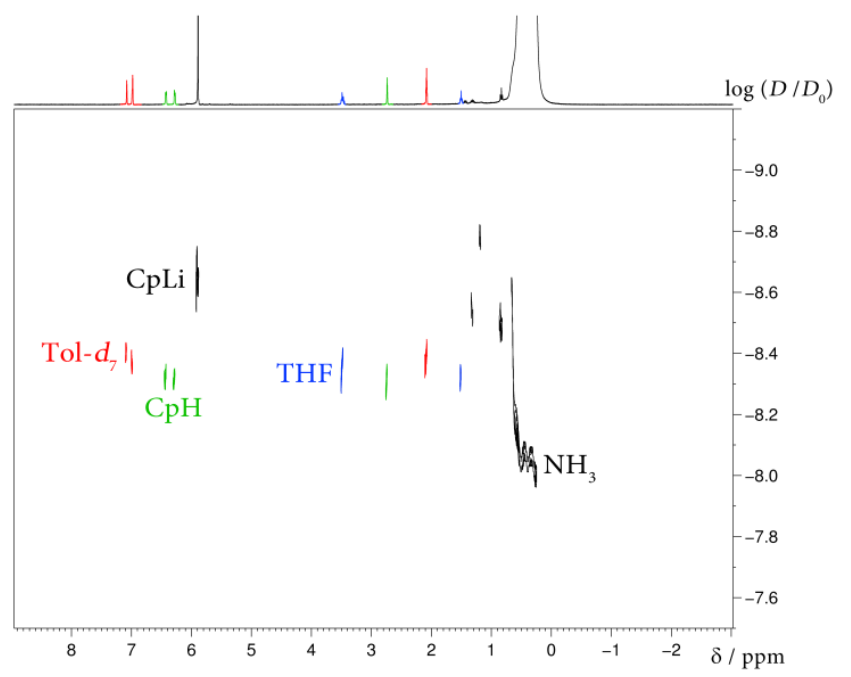

Figure 5-9: ${ }^{1} \mathrm{H}-\mathrm{DOSY}$ NMR spectrum of CpLi in ammonia at $25^{\circ} \mathrm{C}$.

\section{${ }^{7} \mathrm{Li},{ }^{1} \mathrm{H}-\mathrm{HOESY}$ NMR Spectrum of CpLi in DMSO}

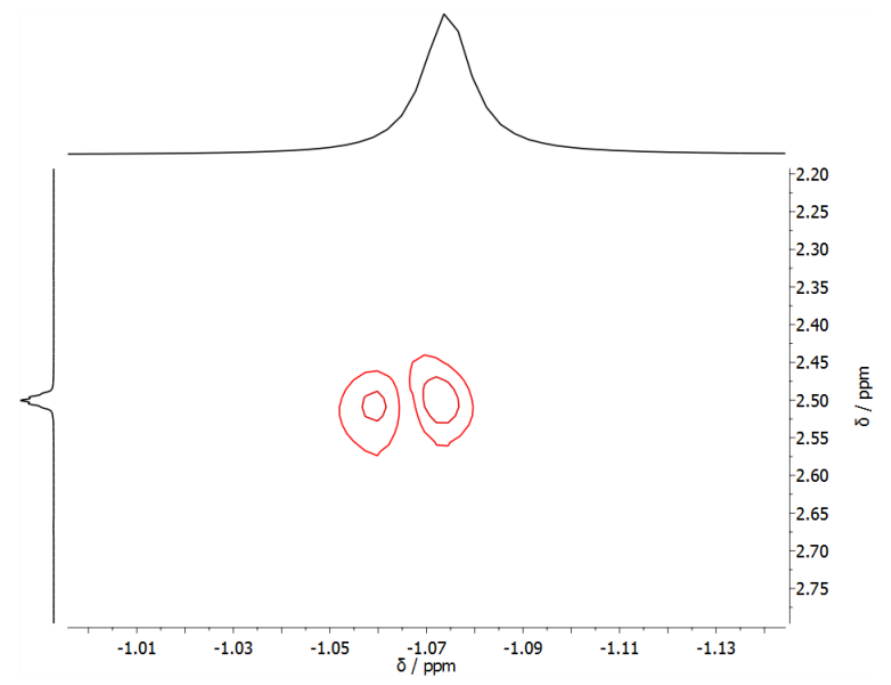

Figure 5-10: ${ }^{7} \mathrm{Li},{ }^{1} \mathrm{H}-\mathrm{HOESY}$ NMR spectrum of CpLi in DMSO- $d_{6}$ at $25^{\circ} \mathrm{C}$. 


\section{REFERENCES}

[1] A. Visscher, S. Bachmann, C. Schnegelsberg, T. Teuteberg, R. A. Mata, D. Stalke, Dalton Trans. 2016, 45, 5689-5699.

[2] S. Bachmann, R. Neufeld, M. Dzemski, D. Stalke, Chem. Eur. J. 2016, 22, 8462-8465.

[3] C. Schnegelsberg, S. Bachmann, M. Kolter, T. Auth, M. John, D. Stalke, K. Koszinowski, Chem. Eur. J. 2016, 22, 7752-7762.

[4] S. Bachmann, B. Gernert, D. Stalke, Chem. Commun. 2016, 52, 12861-12864.

[5] A. Kreyenschmidt, S. Bachmann, T. Niklas, D. Stalke, in preparation.

[6] S. Bachmann, T. Niklas, M. John, D. Stalke, in preparation.

[7] a) S. J. Blundell, F. L. Pratt, J. Phys.: Condens. Matter 2004, 16, R771; b) C. Elschenbroich, Organometallchemie, Vol. 6, Vieweg+Teubner Verlag, Wiesbaden, 2008.

[8] a) P. Beak, A. Basu, D. J. Gallagher, Y. S. Park, S. Thayumanavan, Acc. Chem. Res. 1996, 29, 552-560; b) M. Schlosser, Organoalkali Chemistry, 2nd ed., John Wiley \& Sons Ltd., Chichester, 2004.

[9] W. Schlenk, J. Holtz, Ber. d. chem. Ges. 1917, 50, 262-274.

[10] a) B. J. Wakefield, in The Chemistry of Organolithium Compounds, Pergamon Press, Oxford, New York, 1974; b) J. Clayden, Organolithiums: Selectivity for Synthesis, Vol. 23, Elsevier Science (Pergamon), Oxford, 2002; c) G. Wu, M. Huang, Chem. Rev. 2006, 106, 2596-2616.

[11] H. Dietrich, Acta Crystallogr. Sect. E: Struct. Rep. 1963, 16, 681-689.

[12] T. Kottke, D. Stalke, Angew. Chem. 1993, 105, 619-621; Angew. Chem. Int. Ed. 1993, 32, 580-582.

[13] U. Siemeling, T. Redecker, B. Neumann, H.-G. Stammler, J. Am. Chem. Soc. 1994, 116, 5507-5508.

[14] E. Weiss, G. Hencken, J. Organomet. Chem. 1970, 21, 265-268.

[15] M. A. Nichols, P. G. Williard, J. Am. Chem. Soc. 1993, 115, 1568-1572.

[16] U. Schümann, J. Kopf, E. Weiss, Angew. Chem. 1985, 97, 222-223; Angew. Chem. Int. Ed. 1985, 24, 215216.

[17] a) T. Stey, D. Stalke, in The chemistry of organolithium compounds (Eds.: Z. Rappoport, I. Marek), John Wiley \& Sons, Chichester, 2004; b) E. Carl, D. Stalke, in Lithium Compounds in Organic Synthesis From Fundamentals to Applications (Eds.: R. Luisi, V. Capriati), Wiley-VCH, Weinheim, 2014, 1-13.

[18] A. C. Jones, A. W. Sanders, M. J. Bevan, H. J. Reich, J. Am. Chem. Soc. 2007, 129, 3492-3493.

[19] J. F. McGarrity, J. Prodolliet, T. Smyth, Org. Magn. Res. 1981, 17, 59-65.

[20] J. F. McGarrity, C. A. Ogle, Z. Brich, H. R. Loosli, J. Am. Chem. Soc. 1985, 107, 1810-1815. 
[21] C. A. Ogle, H. C. Johnson, X. L. Wang, F. H. Strickler, D. Bucca, B. Gordon, III, Macromolecules 1995 , $28,5184-5191$

[22] R. O. Ebewele, Polymer Science and Technology, CRC Press, Boca Raton - New York, 2000.

[23] P. Zhao, D. B. Collum, J. Am. Chem. Soc. 2003, 125, 4008-4009.

[24] D. B. Collum, Acc. Chem. Res. 1992, 25, 448-454.

[25] B. O. T. Kammermeier, G. W. Klumpp, K. Kolthof, M. Vos, Tetrahedron Lett. 1991, 32, 3111-3114.

[26] a) G. Wittig, F. J. Meyer, G. Lange, Liebigs Ann. Chem. 1951, 571, 167-201; b) G. Wittig, Angew. Chem. 1958, 70, 65-71; c) G. Wittig, Chem. Soc. Rev. 1966, 20, 191-210.

[27] a) A. C. Jones, A. W. Sanders, W. H. Sikorski, K. L. Jansen, H. J. Reich, J. Am. Chem. Soc. 2008, 130, 6060-6061; b) H. J. Reich, W. H. Sikorski, A. W. Sanders, A. C. Jones, K. N. Plessel, J. Org. Chem. 2009, 74, 719-729.

[28] C. Eaborn, P. B. Hitchcock, J. D. Smith, A. C. Sullivan, Chem. Commun. 1983, 0, 827-828.

[29] a) M. M. Olmstead, P. P. Power, J. Am. Chem. Soc. 1985, 107, 2174-2175; b) A. D. Pajerski, M. Parvez, H. G. Richey, J. Am. Chem. Soc. 1988, 110, 2660-2662; c) S. Buchholz, K. Harms, M. Marsch, W. Massa, G. Boche, Angew. Chem. 1989, 101, 57-58; Angew. Chem. Int. Ed. 1989, 28, 72-73; d) B. Becker, V. Enkelmann, K. Müllen, Angew. Chem. 1989, 101, 501-503; Angew. Chem. Int. Ed. 1989, 28, 458-460; e) H. Gornitzka, D. Stalke, Organometallics 1994, 13, 4398-4405; f) F. Zaegel, J. C. Gallucci, P. Meunier, B. Gautheron, M. R. Sivik, L. A. Paquette, J. Am. Chem. Soc. 1994, 116, 6466-6467; g) H. Bock, A. John, C. Arad, C. Näther, Z. Havlas, Angew. Chem. 1994, 106, 931-934; Angew. Chem. Int. Ed. 1994, 33, 931-933; h) H. Viebrock, U. Behrens, E. Weiss, Angew. Chem. 1994, 106, 1364-1365; Angew. Chem. Int. Ed. 1994, 33, 1257-1259; i) H. Viebrock, D. Abeln, E. Weiss, Z. Naturforsch., B Chem. Sci. 1994, 49, 89-99; j) R. Haag, B. Ohlhorst, M. Noltemeyer, R. Fleischer, D. Stalke, A. Schuster, D. Kuck, A. de Meijere, J. Am. Chem. Soc. 1995, 117, 10474-10485; k) W. A. Herrmann, O. Runte, G. Artus, J. Organomet. Chem. 1995, 501, C1-C4; 1) C. Näther, H. Bock, Z. Havlas, T. Hauck, Organometallics 1998, 17, 4707-4715; m) J. S. Alexander, K. Ruhlandt-Senge, Angew. Chem. 2001, 113, 2732-2734; Angew. Chem. Int. Ed. 2001, 40, 2658-2660; n) S. Harder, F. Feil, T. Repo, Chem. Eur. J. 2002, 8, 1991-1999; o) R. Michel, R. HerbstIrmer, D. Stalke, Organometallics 2010, 29, 6169-6171; p) R. Michel, T. Nack, R. Neufeld, J. M. Dieterich, R. A. Mata, D. Stalke, Angew. Chem. 2013, 125, 762-766; Angew. Chem. Int. Ed. 2013, 52, 734-738; q) A.-C. Pöppler, M. Granitzka, R. Herbst-Irmer, Y.-S. Chen, B. B. Iversen, M. John, R. A. Mata, D. Stalke, Angew. Chem. 2014, 126, 13498-13503; Angew. Chem. Int. Ed. 2014, 53, 13282-13287.

[30] a) J. Smid, Angew. Chem. 1972, 84, 127-144; Angew. Chem. Int. Ed. 1972, 11, 112-127; b) A. Streitwieser, Acc. Chem. Res. 1984, 17, 353-357.

[31] a) A. I. Vogel, A. R. Tachtell, B. S. Furnis, A. J. Hannaford, P. W. G. Smith, Vogel's Textbook of Practical Organic Chemistry, Vol. 5, Prentice Hall, 1989; b) D. R. Lide, CRC Handbook of Chemistry and Physics, Vol. 87, CRC Press, Boca Raton, 2006.

[32] a) A. V. Yakimansky, A. H. E. Muller, M. Van Beylen, Macromolecules 2000, 33, 5686-5692; b) M. Granitzka, Aggregation Study on Lithiated Five-Membered Heterocycles - Towards The Pentuple Ion, Ph.D. thesis, Göttingen, 2013; c) A.-C. Pöppler, Advanced NMR Methodology for the Investigation of Organometallic Compounds in Solution, Ph.D. thesis, Göttingen, 2013.

[33] a) P. Jutzi, in Adv. Organomet. Chem., Vol. 26 (Eds.: F. G. A. Stone, W. Robert), Academic Press, 1986, 217-295; b) P. Jutzi, J. Organomet. Chem. 1990, 400, 1-17; c) E. Weiss, Angew. Chem. 1993, 105, 1565- 
1587; Angew. Chem. Int. Ed. 1993, 32, 1501-1523; d) D. Stalke, Angew. Chem. 1994, 106, 2256-2259; Angew. Chem. Int. Ed. 1994, 33, 2168-2171; e) S. Harder, Coord. Chem. Rev. 1998, 176, 17-66.

[34] a) T. J. Kealy, P. L. Pauson, Nature 1951, 168, 1039-1040; b) G. Wilkinson, R. B. Woodward, J. Am. Chem. Soc. 1952, 74, 2125.

[35] J. Thiele, Ber.d. chem. Ges. 1900, 33, 666-673.

[36] J. Thiele, Ber. d. chem. Ges. 1901, 34, 68-71.

[37] a) H. E. Roscoe, Liebigs Ann. Chem. 1886, 232, 348-352; b) G. Kraemer, A. Spilker, Ber. Dtsch. Chem. Ges. 1896, 29, 552 .

[38] a) E. O. Fischer, R. Jira, K. Hafner, Z. Naturforsch., B: Chem. Sci. 1953, 8, 327; b) E. O. Fischer, W. Hafner, H. O. Stahl, Z. Anorg. Allg. Chem. 1955, 282, 47-62.

[39] K. Ziegler, H. Froitzheim-Kühlhorn, K. Hafner, Chem. Ber. 1956, 89, 434-443.

[40] N. J. Long, Metallocenes: An Introduction to Sandwich Complexes, Wiley-Blackwell, 1997.

[41] J. E. Huheey, E. A. Keiter, R. L. Keiter, Anorganische Chemie - Prinzipien von Struktur und Reaktivität, Vol. 3, Walter de Gruyter, Berlin, 2003.

[42] H. Sinn, W. Kaminsky, H.-J. Vollmer, R. Woldt, Angew. Chem. 1980, 92, 396-402; Angew. Chem. Int. Ed. 1980, 19, 390-392.

[43] a) W. Kaminsky, M. Miri, H. Sinn, R. Woldt, Macromol. Rapid Commun. 1983, 4, 417-421; b) J. Herwig, W. Kaminsky, Polym. Bull. 1983, 9, 464-469; c) W. Kaminsky, Dalton Trans. 1998, 1413-1418.

[44] B. Ye, N. Cramer, Angew. Chem. 2014, 126, 8030-8033; Angew. Chem. Int. Ed. 2014, 53, 7896-7899.

[45] M. Sawamura, Y. Kuninobu, M. Toganoh, Y. Matsuo, M. Yamanaka, E. Nakamura, J. Am. Chem. Soc. 2002, 124, 9354-9355.

[46] B. Ye, N. Cramer, Science 2012, 338, 504-506.

[47] L. Y. Kuo, M. G. Kanatzidis, M. Sabat, A. L. Tipton, T. J. Marks, J. Am. Chem. Soc. 1991, 113, 9027-9045.

[48] P. Jutzi, N. Burford, Chem. Rev. 1999, 99, 969-990.

[49] a) H. P. Fritz, R. Schneider, Chem. Ber. 1960, 93, 1171-1183; b) H. P. Fritz, L. Schäfer, Chem. Ber. 1964, 97, 1829-1833.

[50] a) W. T. Ford, J. Organomet. Chem. 1971, 32, 27-33; b) R. H. Cox, H. W. Terry, L. W. Harrison, J. Am. Chem. Soc. 1971, 93, 3297-3298; c) P. Fischer, J. Stadelhofer, J. Weidlein, J. Organomet. Chem. 1976, $116,65-73$.

[51] V. M. Rayón, G. Frenking, Chem. Eur. J. 2002, 8, 4693-4707.

[52] a) T. Aoyagi, H. M. M. Shearer, K. Wade, G. Whitehead, Chem. Commun. 1976, 164-165; b) T. Aoyagi, H. M. M. Shearer, K. Wade, G. Whitehead, J. Organomet. Chem. 1979, 175, 21-31. 
[53] a) V. Jordan, U. Behrens, F. Olbrich, E. Weiss, J. Organomet. Chem. 1996, 517, 81-88; b) M. L. Cole, C. Jones, P. C. Junk, Dalton Trans. 2002, 896-905; c) W. J. Evans, D. G. Giarikos, J. W. Ziller, J. Organomet. Chem. 2003, 688, 200-205.

[54] R. E. Dinnebier, U. Behrens, F. Olbrich, Organometallics 1997, 16, 3855-3858.

[55] a) R. E. Dinnebier, F. Olbrich, S. VanSmaalen, P. W. Stephens, Acta Crystallogr., Sect. B: Struct. Sci. 1997, 53, 153-158; b) R. E. Dinnebier, F. Olbrich, G. M. Bendele, Acta Crystallogr., Sect. C: Cryst. Struct. Commun. 1997, 53, 699-701.

[56] a) P. Jutzi, W. Leffers, B. Hampel, S. Pohl, W. Saak, Angew. Chem. 1987, 99, 563-564; Angew. Chem. Int. Ed. 1987, 26, 583-584; b) W. J. Evans, T. J. Boyle, J. W. Ziller, Organometallics 1992, 11, 3903-3907; c) R. E. Dinnebier, M. Schneider, S. van Smaalen, F. Olbrich, U. Behrens, Acta Crystallogr., Sect. B: Struct. Sci. 1999, 55, 35-44; d) C. Tedesco, R. E. Dinnebier, F. Olbrich, S. van Smaalen, Acta Crystallogr., Sect. B: Struct. Sci. 2001, 57, 673-679; e) S. Kheradmandan, H. W. Schmalle, H. Jacobsen, O. Blacque, T. Fox, H. Berke, M. Gross, S. Decurtins, Chem. Eur. J. 2002, 8, 2526-2533; f) G. Bai, H. W. Roesky, P. Müller, Bull. Pol. Acad. Sci. Chem. 2002, 50, 1; g) U. Behrens, R. E. Dinnebier, S. Neander, F. Olbrich, Organometallics 2008, 27, 5398-5400.

[57] P. Jutzi, E. Schlüter, C. Krüger, S. Pohl, Angew. Chem. 1983, 95, 1015-1016; Angew. Chem. Int. Ed. 1983, 22,994

[58] a) M. F. Lappert, A. Singh, L. M. Engelhardt, A. H. White, J. Organomet. Chem. 1984, 262, 271-278; b) P. Jutzi, E. Schlüter, S. Pohl, W. Saak, Chem. Ber. 1985, 118, 1959-1967.

[59] a) P. Jutzi, W. Leffers, S. Pohl, W. Saak, Chem. Ber. 1989, 122, 1449-1456; b) A. Hammel, W. Schwarz, J. Weidlein, Acta Crystallogr. C 1990, 46, 2337-2339; c) G. Rabe, H. W. Roesky, D. Stalke, F. Pauer, G. M. Sheldrick, J. Organomet. Chem. 1991, 403, 11-19; d) H. Chen, P. Jutzi, W. Leffers, M. M. Olmstead, P. P. Power, Organometallics 1991, 10, 1282-1286; e) J. Hiermeier, F. H. Koehler, G. Mueller, Organometallics 1991, 10, 1787-1793; f) A. Sekiguchi, Y. Sugai, K. Ebata, C. Kabuto, H. Sakurai, J. Am. Chem. Soc. 1993, 115, 1144-1146; g) M. A. Edelman, P. B. Hitchcock, M. F. Lappert, D.-S. Liu, S. Tian, J. Organomet. Chem. 1998, 550, 397-408; h) S. Neander, F. E. Tio, R. Buschmann, U. Behrens, F. Olbrich, J. Organomet. Chem. 1999, 582, 58-65; i) S. Neander, U. Behrens, F. Olbrich, J. Organomet. Chem. 2000, 604, 59-67; j) T. Kähler, U. Behrens, S. Neander, F. Olbrich, J. Organomet. Chem. 2002, 649, 50-54; k) W. J. Evans, J. C. Brady, C. H. Fujimoto, D. G. Giarikos, J. W. Ziller, J. Organomet. Chem. 2002, 649, 252-257; 1) H. Schumann, M. R. Keitsch, S. H. Mühle, Z. Anorg. Allg. Chem. 2002, 628, 1311-1318; m) C. M. Widdifield, J. A. Tang, C. L. B. Macdonald, R. W. Schurko, Magn. Reson. Chem. 2007, 45, S116-S128; n) R. Michel, R. Herbst-Irmer, D. Stalke, Organometallics 2011, 30, 4379-4386; o) J. Hey, D. M. Andrada, R. Michel, R. A. Mata, D. Stalke, Angew. Chem. 2013, 125, 10555-10559; Angew. Chem. Int. Ed. 2013, 52, 10365-10369; p) J. Hey, From X-ray diffraction data annealing to comprehensive charge density analysis, Ph. D. thesis, Göttingen, 2013; q) C. B. Benda, M. Waibel, T. F. Fässler, Angew. Chem. 2015, 127, 532-536; Angew. Chem. Int. Ed., 2015, 54, 522-526; r) F. Ortu, J. M. Fowler, M. Burton, A. Formanuik, D. P. Mills, New J. Chem. 2015, 39, 7633-7639.

[60] a) G. Lin, W.-T. Wong, Polyhedron 1994, 13, 3027-3030; b) C. Dohmeier, E. Baum, A. Ecker, R. Köppe, H. Schnöckel, Organometallics 1996, 15, 4702-4706.

[61] A. A. Fyfe, A. R. Kennedy, J. Klett, R. E. Mulvey, Angew. Chem. 2011, 123, 7922-7926; Angew. Chem. Int. Ed. 2011, 50, 7776-7780.

[62] S. Harder, M. H. Prosenc, Angew. Chem. 1994, 106, 1830-1832; Angew. Chem. Int. Ed. 1994, 33, 17441746. 
[63] J. Wessel, E. Lork, R. Mews, Angew. Chem. 1995, 107, 2565-2567; Angew. Chem. Int. Ed., 1995, 34, 2376-2378.

[64] S. Harder, M. H. Prosenc, U. Rief, Organometallics 1996, 15, 118-122.

[65] M. Westerhausen, S. Schneiderbauer, N. Makropoulos, M. Warchhold, H. Nöth, H. Piotrowski, K. Karaghiosoff, Organometallics 2002, 21, 4335-4341.

[66] L. A. Paquette, W. Bauer, M. R. Sivik, M. Buehl, M. Feigel, P. v. R. Schleyer, J. Am. Chem. Soc. 1990, 112, 8776-8789.

[67] R. den Besten, S. Harder, L. Brandsma, J. Organomet. Chem. 1990, 385, 153-159.

[68] a) S. D. Stults, R. A. Andersen, A. Zalkin, J. Am. Chem. Soc. 1989, 111, 4507-4508; b) J.-C. Berthet, C. Villiers, J.-F. Le Maréchal, B. Delavaux-Nicot, M. Lance, M. Nierlich, J. Vigner, M. Ephritikhine, J. Organomet. Chem. 1992, 440, 53-65; c) W. J. Evans, M. S. Sollberger, J. L. Shreeve, J. M. Olofson, J. H. Hain, J. W. Ziller, Inorg. Chem. 1992, 31, 2492-2501.

[69] S. Harder, M. H. Prosenc, Angew. Chem. 1996, 108, 101-103; Angew. Chem. Int. Ed. 1996, 35, 97-99.

[70] a) D. Seebach, R. Amstutz, T. Laube, W. B. Schweizer, J. D. Dunitz, J. Am. Chem. Soc. 1985, 107, 54035409; b) H. Hope, Acta Crystallogr., Sect. B: Struct. Sci. 1988, 44, 22-26; c) R. Boese, D. Blaser, J. Appl. Crystallogr. 1989, 22, 394-395; d) T. Kottke, D. Stalke, J. Appl. Crystallogr. 1993, 26, 615-619; e) A. E. H. Wheatley, Chem. Soc. Rev. 2001, 30, 265-273.

[71] a) T. Niklas, D. Stalke, M. John, Chem. Commun. 2015, 51, 1275-1277; b) T. Niklas, C. Steinmetzger, W. Liu, D. Zell, D. Stalke, L. Ackermann, M. John, Eur. J. Org. Chem. 2015, 2015, 6801-6805.

[72] a) J. Heinzer, J. F. M. Oth, D. Seebach, Helv. Chim. Acta 1985, 68, 1848-1862; b) T. Claridge, HighResolution NMR Techniques in Organic Chemistry, Vol. 27, Elsevier, 2009; c) J. Keeler, Understanding NMR Spectroscopy, 2nd ed., John Wiley \& Sons, 2010; d) H. Friebolin, Basic One- and Two-Dimensional NMR Spectroscopy, 5th ed., WILEY-VCH Verlag, Weinheim, 2011.

[73] C. Yu, G. C. Levy, J. Am. Chem. Soc. 1983, 105, 6994-6996.

[74] P. L. Rinaldi, J. Am. Chem. Soc. 1983, 105, 5167-5168.

[75] W. Bauer, G. Müller, R. Pi, P. von Ragué Schleyer, Angew. Chem. 1986, 98, 1130-1132; Angew. Chem. Int. Ed. 1986, 25, 1103-1104.

[76] M. Granitzka, A.-C. Pöppler, E. K. Schwarze, D. Stern, T. Schulz, M. John, R. Herbst-Irmer, S. K. Pandey, D. Stalke, J. Am. Chem. Soc. 2011, 134, 1344-1351.

[77] F. T. Edelmann, F. Knösel, F. Pauer, D. Stalke, W. Bauer, J. Organomet. Chem. 1992, 438, 1-10.

[78] Bruker, Almanac 2014, 1st ed., 2014.

[79] a) W. Bauer, Magn. Reson. Chem. 1991, 29, 494-499; b) D. Hoffmann, W. Bauer, P. v. R. Schleyer, U. Pieper, D. Stalke, Organometallics 1993, 12, 1193-1200.

[80] a) P. Stilbs, Prog. Nucl. Magn. Res. Spectr. 1987, 19, 1-45; b) C. S. Johnson Jr, Prog. Nucl. Magn. Res. Spectr. 1999, 34, 203-256; c) Y. Cohen, L. Avram, L. Frish, Angew. Chem. 2005, 117, 524-560; Angew. Chem. Int. Ed. 2005, 44, 520-554; d) A. Macchioni, G. Ciancaleoni, C. Zuccaccia, D. Zuccaccia, Chem. 
Soc. Rev. 2008, 37, 479-489; e) T. D. W. Claridge, High-Resolution NMR Techniques in Organic Chemistry, Vol. 27, Elsevier, 2009; f) L. Avram, Y. Cohen, Chem. Soc. Rev. 2015, 44, 586-602.

[81] E. O. Stejskal, J. E. Tanner, J. Chem. Phys. 1965, 42, 288-292.

[82] E. L. Hahn, Phys. Rev. 1950, 80, 580-594.

[83] J. E. Tanner, J. Chem. Phys. 1970, 52, 2523-2526.

[84] a) S. J. Gibbs, C. S. Johnson, J. Magn. Reson. 1991, 93, 395-402; b) D. H. Wu, A. D. Chen, C. S. Johnson, J. Magn. Reson., Ser. A 1995, 115, 260-264.

[85] A. Jerschow, N. Müller, J. Magn. Reson. 1996, 123, 222-225.

[86] a) K. Zangger, H. Sterk, J. Magn. Reson. 1997, 124, 486-489; b) J. A. Aguilar, S. Faulkner, M. Nilsson, G. A. Morris, Angew. Chem. 2010, 122, 3993-3995; Angew. Chem. Int. Ed. 2010, 49, 3901-3903; c) R. W. Adams, L. Byrne, P. Kiraly, M. Foroozandeh, L. Paudel, M. Nilsson, J. Clayden, G. A. Morris, Chem. Commun. 2014, 50, 2512-2514.

[87] G. Hamdoun, M. Sebban, E. Cossoul, A. Harrison-Marchand, J. Maddaluno, H. Oulyadi, Chem. Commun. 2014, 50, 4073-4075.

[88] K. F. Morris, C. S. Johnson, J. Am. Chem. Soc. 1992, 114, 3139-3141.

[89] J. S. Gounarides, A. Chen, M. J. Shapiro, J. Chromatogr. B. 1999, 725, 79-90.

[90] a) S. Beck, A. Geyer, H.-H. Brintzinger, Chem. Commun. 1999, 2477-2478; b) M. Valentini, P. S. Pregosin, H. Rüegger, Organometallics 2000, 19, 2551-2555; c) A. Pichota, P. S. Pregosin, M. Valentini, M. Wörle, D. Seebach, Angew. Chem. 2000, 112, 157-160; Angew. Chem. Int. Ed. 2000, 39, 153-156.

[91] I. Keresztes, P. G. Williard, J. Am. Chem. Soc. 2000, 122, 10228-10229.

[92] a) M. Nilsson, A. M. Gil, I. Delgadillo, G. A. Morris, Chem. Commun. 2005, 1737-1739; b) B. Vitorge, D. Jeanneat, Anal. Chem. 2006, 78, 5601-5606.

[93] M. D. Pluth, K. N. Raymond, Chem. Soc. Rev. 2007, 36, 161-171.

[94] J. T. Edward, J. Chem. Educ. 1970, 47, 261.

[95] A. Gierer, K. Wirtz, Z. Naturforsch., A: Phys. Sci. 1953, 8, 532.

[96] H. C. Chen, S. H. Chen, J. Phys. Chem. 1984, 88, 5118-5121.

[97] F. Perrin, J. Phys. Radium 1936, 7, 1-11.

[98] R. Evans, Z. Deng, A. K. Rogerson, A. S. McLachlan, J. J. Richards, M. Nilsson, G. A. Morris, Angew. Chem. 2013, 125, 3281-3284; Angew. Chem. Int. Ed. 2013, 52, 3199-3202.

[99] S. Augé, P.-O. Schmit, C. A. Crutchfield, M. T. Islam, D. J. Harris, E. Durand, M. Clemancey, A.-A. Quoineaud, J.-M. Lancelin, Y. Prigent, F. Taulelle, M.-A. Delsuc, J. Phys. Chem. B 2009, 113, 1914-1918.

[100] P. Flory, Principles of Polymer Chemistry, Cornell University Press, Ithaca, New York, 1953. 
[101] a) A. Chen, D. Wu, C. S. Johnson, J. Am. Chem. Soc. 1995, 117, 7965-7970; b) K. Chari, B. Antalek, J. Minter, Phys. Rev. Lett. 1995, 74, 3624-3627.

[102] S. Floquet, S. Brun, J.-F. Lemonnier, M. Henry, M.-A. Delsuc, Y. Prigent, E. Cadot, F. Taulelle, J. Am. Chem. Soc. 2009, 131, 17254-17259.

[103] a) D. K. Wilkins, S. B. Grimshaw, V. Receveur, C. M. Dobson, J. A. Jones, L. J. Smith, Biochemistry 1999, 38, 16424-16431; b) S. Viel, D. Capitani, L. Mannina, A. Segre, Biomacromolecules 2003, 4, 1843 1847.

[104] C. A. Crutchfield, D. J. Harris, J. Magn. Reson. 2007, 185, 179-182.

[105] a) J. A. Jones, D. K. Wilkins, L. J. Smith, C. M. Dobson, J. Biomol. NMR 1997, 10, 199-203; b) E. J. Cabrita, S. Berger, Magn. Reson. Chem. 2001, 39, S142-S148.

[106] a) D. Li, I. Keresztes, R. Hopson, P. G. Williard, Acc. Chem. Res. 2009, 42, 270-280; b) D. Li, G. Kagan, R. Hopson, P. G. Williard, J. Am. Chem. Soc. 2009, 131, 5627-5634.

[107] a) D. Li, C. Sun, P. G. Williard, J. Am. Chem. Soc. 2008, 130, 11726-11736; b) A. M. Socha, G. Kagan, W. Li, R. Hopson, J. K. Sello, P. G. Williard, Energy Fuels 2010, 24, 4518-4521; c) D. R. Armstrong, P. García-Álvarez, A. R. Kennedy, R. E. Mulvey, S. D. Robertson, Chem. Eur. J. 2011, 17, 6725-6730; d) P. García-Álvarez, R. E. Mulvey, J. A. Parkinson, Angew. Chem. 2011, 123, 9842-9845; Angew. Chem. Int. Ed. 2011, 50, 9668-9671; e) C. Su, R. Hopson, P. G. Williard, J. Am. Chem. Soc. 2013, 135, 12400-12406; f) C. Su, R. Hopson, P. G. Williard, Eur. J. Inorg. Chem. 2013, 2013, 4136-4141; g) C. Su, R. Hopson, P. G. Williard, J. Am. Chem. Soc. 2013, 135, 14367-14379; h) D. R. Armstrong, A. R. Kennedy, R. E. Mulvey, S. D. Robertson, Dalton Trans. 2013, 42, 3704-3711; i) A. J. Martinez-Martinez, D. R. Armstrong, B. Conway, B. J. Fleming, J. Klett, A. R. Kennedy, R. E. Mulvey, S. D. Robertson, C. T. O'Hara, Chem. Sci. 2014, 5, 771-781; j) S. C. Hunter, S.-J. Chen, C. A. Steren, M. G. Richmond, Z.-L. Xue, Organometallics 2015, 34, 5687-5696.

[108] a) D. Li, R. Hopson, W. Li, J. Liu, P. G. Williard, Org. Lett. 2008, 10, 909-911; b) G. Kagan, W. Li, R. Hopson, P. G. Williard, Org. Lett. 2009, 11, 4818-4821; c) J. Guang, R. Hopson, P. G. Williard, J. Org. Chem. 2015, 80, 9102-9107.

[109] a) R. Neufeld, D. Stalke, Chem. Sci. 2015, 6, 3354-3364; b) R. Neufeld, DOSY External Calibration Curve Molecular Weight Determination as a Valuable Methodology in Characterizing Reactive Intermediates in Solution, Ph. D. thesis, Göttingen, 2016.

[110] R. Neufeld, M. John, D. Stalke, Angew. Chem. 2015, 127, 7100-7104; Angew. Chem. Int. Ed. 2015, 54, 6994-6998.

[111] a) R. Neufeld, D. Stalke, Chem. Eur. J. 2016, 22, 12624-12628; b) R. Neufeld, T. L. Teuteberg, R. HerbstIrmer, R. A. Mata, D. Stalke, J. Am. Chem. Soc. 2016, 138, 4796-4806.

[112] M. Á. Fuentes, A. Zabala, A. R. Kennedy, R. E. Mulvey, Chem. Eur. J. 2016, 22, 14968-14978.

[113]Y. Qiao, W. Ge, L. Jia, X. Hou, Y. Wang, C. M. Pedersen, Chem. Commun. 2016, 52, 11418-11421.

[114] A. I. Ojeda-Amador, A. J. Martínez-Martínez, A. R. Kennedy, C. T. O’Hara, Inorg. Chem. 2016, 55, 5719-5728.

[115] R. Neufeld, R. Michel, R. Herbst-Irmer, R. Schöne, D. Stalke, Chem. Eur. J. 2016, 22, 12340-12346. 
[116]J. Wang, T. Hou, J. Comput. Chem. 2011, 32, 3505-3519.

[117]W. M. Haynes, D. R. Lide, CRC Handbook of Chemistry and Physics, Vol. 92, Taylor \& Francis, Boca Raton, 2011.

[118]J. Goubeau, J. Jiménez-Barberá, Z. Anorg. Allg. Chem. 1960, 303, 217-342.

[119] http://macro.lsu.edu/HowTo/solvents/, accessed on 22.10.2016, provided by Louisiana State University.

[120] L. Mak, S. Grandison, R. J. Morris, J. Mol. Graphics Modell. 2008, 26, 1035-1045.

[121]H. Lee, J. R. Baker, R. G. Larson, J. Phys. Chem. B 2006, 110, 4014-4019.

[122] 16a.IT.2016 ed., Institute of Physics, Polish Academy of Sciences, Al. Lotnikow 32/46, Warszawa, Poland, 2016.

[123] a) A. Bondi, J. Phys. Chem. 1964, 68, 441-451; b) R. S. Rowland, R. Taylor, J. Phys. Chem. 1996, 100, 7384-7391; c) M. Mantina, A. C. Chamberlin, R. Valero, C. J. Cramer, D. G. Truhlar, J. Phys. Chem. A 2009, 113, 5806-5812.

[124]A. Kreyenschmidt, Externe Kalibrierungskurven zur Bestimmung des Molekulargewichtes von Verbindungen mit Schweratomen, Master thesis, Göttingen, 2016.

[125] a) R. H. Cox, H. W. Terry Jr, J. Magn. Reson. 1974, 14, 317-322; b) E. Herdtweck, F. H. Köhler, R. Mölle, Eur. J. Inorg. Chem. 2005, 2005, 952-958; c) D. Johnels, A. Boman, U. Edlund, Magn. Reson. Chem. 1998, 36, S151-S156.

[126]J. Mason, Multinuclear NMR, Plenum Press, New York, 1987.

[127] U. Behrens, F. Olbrich, Private Communication, CCDC 6872632008.

[128] M. Kaupp, P. v. R. Schleyer, J. Phys. Chem. 1992, 96, 7316-7323.

[129] U. Pieper, D. Stalke, Organometallics 1993, 12, 1201-1206.

[130] S. Harvey, C. L. Raston, B. W. Skelton, A. H. White, M. F. Lappert, G. Srivastava, J. Organomet. Chem. 1987, 328, C1-C6.

[131] O. T. Beachley, R. Blom, M. R. Churchill, K. Faegri, J. C. Fettinger, J. C. Pazik, L. Victoriano, Organometallics 1989, 8, 346-356.

[132] A. I. Popov, Pure Appl. Chem. 1975, 41, 275.

[133]W. J. DeWitte, L. Liu, E. Mei, J. L. Dye, A. I. Popov, J. Solution Chem. 1977, 6, 337-348.

[134]J. W. Strauch, J.-L. Fauré, S. Bredeau, C. Wang, G. Kehr, R. Fröhlich, H. Luftmann, G. Erker, J. Am. Chem. Soc. 2004, 126, 2089-2104.

[135]V. Grignard, Comptes Rendus Hebdomadaires de la Seances de l'Academie des Sciences 1900, 130, 13221324.

[136] a) K. Ziegler, Angew. Chem. 1957, 69, 657-657; b) E. C. Ashby, Chem. Soc. Rev. 1967, 21, 259-285; c) M. Tamura, J. K. Kochi, J. Am. Chem. Soc. 1971, 93, 1487-1489; d) G. Fouquet, M. Schlosser, W. Malisch, M. Kuhn, Angew. Chem. 1974, 86, 50-51; Angew. Chem. Int. Ed. 1974, 13, 82-83; e) P. Knochel, W. 
Dohle, N. Gommermann, F. F. Kneisel, F. Kopp, T. Korn, I. Sapountzis, V. A. Vu, Angew. Chem. 2003, 115, 4438-4456; Angew. Chem. Int. Ed. 2003, 42, 4302-4320; f) D. Martin, S. Kehrli, M. d'Augustin, H. Clavier, M. Mauduit, A. Alexakis, J. Am. Chem. Soc. 2006, 128, 8416-8417; g) S. R. Harutyunyan, T. den Hartog, K. Geurts, A. J. Minnaard, B. L. Feringa, Chem. Rev. 2008, 108, 2824-2852; h) D. Seyferth, Organometallics 2009, 28, 1598-1605.

[137] K. C. Nicolaou, M. Takayanagi, N. F. Jain, S. Natarajan, A. E. Koumbis, T. Bando, J. M. Ramanjulu, Angew. Chem. 1998, 110, 2881-2883; Angew. Chem. Int. Ed. 1998, 37, 2717-2719.

[138] H. G. Richey, Grignard reagents: new developments, Wiley, 2000.

[139]W. Schlenk, W. Schlenk, Ber. d. chem. Ges. 1929, 62, 920-924.

[140]a) R. M. Salinger, H. S. Mosher, J. Am. Chem. Soc. 1964, 86, 1782-1786; b) M. B. Smith, W. E. Becker, Tetrahedron 1967, 23, 4215-4227; c) F. W. Walker, E. C. Ashby, J. Am. Chem. Soc. 1969, 91, 3845-3850; d) T. Holm, Acta Chem. Scand. 1969, 23, 579-586; e) E. C. Ashby, G. E. Parris, J. Am. Chem. Soc. 1971, 93, 1206-1213; f) E. C. Ashby, Pure Appl. Chem. 1980, 52, 545-569; g) R. Benn, H. Lehmkuhl, K. Mehler, A. Rufińska, Angew. Chem. 1984, 96, 521-523; Angew. Chem. Int. Ed. 1984, 23, 534-535; h) T. S. Ertel, H. Bertagnolli, Polyhedron 1993, 12, 2175-2184.

[141] a) E. C. Ashby, W. E. Becker, J. Am. Chem. Soc. 1963, 85, 118-119; b) F. Blasberg, M. Bolte, M. Wagner, H.-W. Lerner, Organometallics 2012, 31, 1001-1005; c) S. Sakamoto, T. Imamoto, K. Yamaguchi, Org. Lett. 2001, 3, 1793-1795; d) J. Toney, G. D. Stucky, J. Organomet. Chem. 1971, 28, 5-20.

[142] a) A. Krasovskiy, P. Knochel, Angew. Chem. 2004, 116, 3396-3399; Angew. Chem. Int. Ed. 2004, 43, 3333-3336; b) F. Kopp, A. Krasovskiy, P. Knochel, Chem. Commun. 2004, 2288-2289; c) H. Ren, A. Krasovskiy, P. Knochel, Org. Lett. 2004, 6, 4215-4217; d) C. B. Rauhut, V. A. Vu, F. F. Fleming, P. Knochel, Org. Lett. 2008, 10, 1187-1189.

[143] a) D. Hauk, S. Lang, A. Murso, Org. Process Res. Dev. 2006, 10, 733-738; b) F. M. Piller, P. Appukkuttan, A. Gavryushin, M. Helm, P. Knochel, Angew. Chem. 2008, 120, 6907-6911; Angew. Chem. Int. Ed. 2008, 47, 6802-6806; c) F. M. Piller, A. Metzger, M. A. Schade, B. A. Haag, A. Gavryushin, P. Knochel, Chem. Eur. J. 2009, 15, 7192-7202; Chemistry; d) R. Li-Yuan Bao, R. Zhao, L. Shi, Chem. Commun. 2015, 51, 6884-6900.

[144] A. Krasovskiy, B. F. Straub, P. Knochel, Angew. Chem. 2006, 118, 165-189; Angew. Chem. Int. Ed. 2006, $45,159-162$.

[145] S. O. Reichmann, Unconventional Carbene-Donor Ligands for the Development of New Catalysts, Ph. D. thesis, Göttingen, 2016.

[146] A. J. Arduengo, R. L. Harlow, M. Kline, J. Am. Chem. Soc. 1991, 113, 361-363.

[147] G. C. Vougioukalakis, R. H. Grubbs, Chem. Rev. 2010, 110, 1746-1787.

[148]a) O. Schuster, L. Yang, H. G. Raubenheimer, M. Albrecht, Chem. Rev. 2009, 109, 3445-3478; b) E. Aldeco-Perez, A. J. Rosenthal, B. Donnadieu, P. Parameswaran, G. Frenking, G. Bertrand, Science 2009, 326, 556-559; c) G. Ung, G. Bertrand, Chem. Eur. J. 2011, 17, 8269-8272.

[149] a) N. Wellinghausen, M. Martin, L. Rink, Eur. J. Immunol. 1997, 27, 2529-2535; b) M. J. Salgueiro, M. Zubillaga, A. Lysionek, M. I. Sarabia, R. Caro, T. De Paoli, A. Hager, R. Weill, J. Boccio, Nutr. Res. 2000, 20, 737-755; c) K. Nogawa, A. Ishizaki, E. Kobayashi, Environ. Res. 1979, 18, 397-409; d) C. Tohyama, Z. A. Shaikh, K. Nogawa, E. Kobayashi, R. Honda, Arch. Toxicol. 1982, 50, 159-166. 
[150]A. Visscher, Fluorescence Studies of Amine-Substituted Azaanthracene Metal Complexes, Ph.D thesis, Göttingen, 2016.

[151] M. Karplus, J. Am. Chem. Soc. 1963, 85, 2870-2871.

[152] a) H. Gornitzka, D. Stalke, Eur. J. Inorg. Chem. 1998, 1998, 311-317; b) T. E. Wood, B. Berno, C. S. Beshara, A. Thompson, J. Org. Chem. 2006, 71, 2964-2971.

[153] a) D.-R. Dauer, D. Stalke, Dalton Trans. 2014, 43, 14432-14439; b) D.-R. Dauer, M. Flügge, R. HerbstIrmer, D. Stalke, Dalton Trans. 2016, 45, 6149-6158; c) D.-R. Dauer, M. Flügge, R. Herbst-Irmer, D. Stalke, Dalton Trans. 2016, 45, 6136-6148.

[154] F. M. Arrabal-Campos, P. Ona-Burgos, I. Fernandez, Polym. Chem. 2016, 7, 4326-4329.

[155] W. G. Kofron, L. M. Baclawski, J. Org. Chem. 1976, 41, 1879-1880.

[156]A. Jerschow, N. Müller, J. Magn. Reson. 1997, 372-375.

[157] G. R. Fulmer, A. J. M. Miller, N. H. Sherden, H. E. Gottlieb, A. Nudelman, B. M. Stoltz, J. E. Bercaw, K. I. Goldberg, Organometallics 2010, 29, 2176-2179.

[158] B. O. Wagner, H. F. Ebel, Tetrahedron 1970, 26, 5155-5167.

[159]J. L. Robbins, N. M. Edelstein, S. R. Cooper, J. C. Smart, J. Am. Chem. Soc. 1979, 101, 3853-3857. 


\section{DANKSAGUNG}

Es gibt viele Menschen, ohne die diese Arbeit nicht oder zumindest nicht in dieser Form zustande gekommen wäre und für all die Mühe und Unterstützung in den vergangenen 3 Jahren möchte ich mich an dieser Stelle bedanken:

Mein besonderer Dank gilt meinem Doktorvater Prof. Dr. Dietmar Stalke, der es mir nicht nur ermöglichte, diese Arbeit in seinem Arbeitskreis zu schreiben, sondern es mir auch sehr leicht gemacht hat, durch das frei wählbare Thema und die vielen Kooperationsmöglichkeiten im und um den Arbeitskreis, diese Arbeit mit Leben zu füllen. Auch für die Möglichkeiten, Chemie auf mehreren Tagungen einmal in einem internationalen Umfeld $\mathrm{zu}$ erleben, möchte ich mich bedanken.

Außerdem möchte ich mich bei meinem Betreuer Dr. Michael John bedanken, der mir bei NMRspektroskopischen Fragestellungen aller Art und vor allem auch an den Maschinen immer mit Rat und Tat zur Seite stand.

Ich danke Prof. Dr. Konrad Koszinowski für die Übernahme des Korreferats und der gesamten Prüfungskommission für die Mühe des Lesens dieser Arbeit.

Ein weiterer Dank gilt auch allen Korrekturlesern dieser Arbeit: Thomas Niklas, Jochen Jung, Alexander Paesch, Anne Kreyenschmidt, Johannes Kretsch und Vivian Bachmann.

Nun folgen die Menschen, durch die der „Arbeitsalltag“ insgesamt immer sehr angenehm war: Zuerst muss ich dabei Roman Neufeld und Thomas Niklas danken, sowohl für die super Atmosphäre durch die ganze Promotion hinweg als auch für das Diskutieren, welches sich nicht nur auf NMR-spektroskopische Fragen beschränkte. Ich erinnere mich immer noch gern an die gemeinsame Zeit auf der Euromar in Prag. Zudem danke ich der gesamten Kickertruppe für die intensiv gefüllten „Denkpausen“ zwischen Messungen und Schreibarbeiten. Ich könnte an dieser Stelle noch viele weitere Namen aus dem Arbeitskreis aufzählen, die die ganze Promotion sehr kurzweilig haben werden lassen, aber um niemanden zu vergessen, möchte ich mich einfach bei allen aus dem Arbeitskreis bedanken: Ihr seid großartig!

Ein weiterer Dank gilt all meinen Kooperationspartnern, insbesondere Christoph „Kroko“ Schnegelsberg, Arne Visscher, Sven Ole Reichmann und Ingo Köhne.

Ein großer Teil der Promotion fand vor den NMR-Geräten statt und die gesamte NMR-Abteilung, insbesondere Christiane Siebert, Martin Weitemeyer und Ralf Schöne, half, dass ich immer besonders gerne meine Zeit vor den Geräten verbracht habe. Zudem möchte ich auch noch Martin Schlote danken für die ganzen Chemikalien und Geräte, die man doch hin und wieder, selbst bei einem NMR-spektroskopischen Thema, benötigte. 
Darüber hinaus möchte ich mich auch bei Ann-Christin Pöppler bedanken, nicht nur für das Korrekturlesen von Arbeiten und Papern, sondern auch dafür, dass ich durch sie den ersten Einblick in die NMR-Spektroskopie bekommen habe, an der ich bis heute gerne arbeite. Auch Anne Kreyenschmidt soll erwähnt werden, die in ihrer Masterarbeit und hoffentlich auch in Zukunft die NMR-Spektroskopie im AK Stalke würdig fortsetzt.

In diesem Sinn möchte ich mich auch bei meinem ACF Praktikanten Kris Bielefeld bedanken für die Synthese so mancher Cp Verbindung.

Meiner Familie und meinen Freunden danke ich für die Zeiten, in denen sie mir durch das gesamte Studium hindurch geholfen und zur Seite gestanden haben.

$\mathrm{Zu}$ guter Letzt danke ich meiner Frau Vivian: Ohne dich wäre all das in dieser Arbeit Zusammengetragene nur halb so schön und auch jede neue Erkenntnis für mich ganz persönlich ziemlich bedeutungslos. Danke, dass du es die ganzen Jahre in Kauf genommen hast, dass meine Zeit doch ziemlich begrenzt gewesen ist. Ich bin mir nicht sicher, wie erfolgreich ich diesen Weg ohne deine fortwährende Unterstützung hätte gehen können.

Ich danke allen von Herzen und hoffe, dass sich auch diejenigen mit Dank versehen fühlen, die ich in dieser kurzen Zusammenfassung nicht namentlich nennen konnte! 


\section{CURRICULUM VitAE}

\section{Sebastian Bachmann}

Richard-Koch-Str. 2

38685 Langelsheim

mobil: +49 (0)151 - 55573622

mail: sbachmann@chemie.uni-goettingen.de

${ }^{\star} 02.09 .1987$ in Goslar

Married

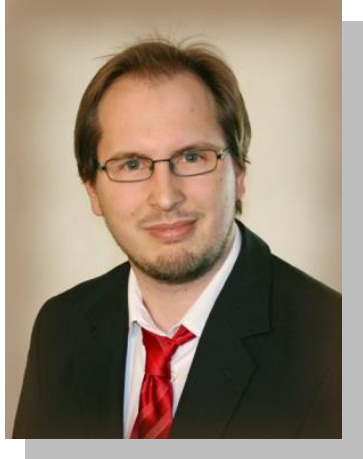

\section{Scientific Career}

$10 / 13$ - today

$10 / 11-09 / 13$

$03 / 13-09 / 13$

$10 / 08-09 / 11$

$03 / 11-06 / 11$

$08 / 00-06 / 07$
Ph.D. thesis „Estimating Molecular Weights of Organometallics in Solution with Diffusion NMR Techniques" with Prof. Dr. D. Stalke and Dr. M. John at the Institute of Inorganic Chemistry at Georg-August-University Göttingen within the Georg-August University School of Science (GAUSS).

Master of Science at Georg-August-University Göttingen passed with grade 2.2 („gut“)

Master thesis under the supervision of Prof. Dr. Stalke „Kinetische Untersuchungen von Aggregaten des Trimethylsilylmethyllithiums mittels NMR-Spektroskopie“ passed with grade 1.7 (,gut")

Bachelor of Science at Georg-August-University Göttingen passed with grade 1.9 („gut“)

Bachelor thesis under the supervision of Prof. Dr. Stalke „NMRspektroskopische Untersuchungen von Amminboran und seinen Derivaten“ passed with grade 1.3 („sehr gut“)

A-level at Ratsgymnasium Goslar (grade 2.3 („gut”))

\section{Experience in University and Abroad}

$10 / 13$ - today

$10 / 13$ - today

$10 / 13$ - today
Scientific researcher with Prof. Dr. D. Stalke

Supervision of practical and theoretical labwork of main subject chemistry students

Lecturer and organizer of the associated seminar and lab course for the first and second year chemistry students as part of their bachelor of science 
Memberships and awards

Sozialdemokratische Partei Deutschlands (SPD)

Gesellschaft Deutscher Chemiker (GdCh)

Student's choice award for „Beste nicht-selbstständige Lehre IAC“ (SS15)

\section{Personal skills}

Languages

German (mother tongue)

English, fluent (ZESS Göttingen, Scientific English I, C1)

Japanese, good (ZESS Göttingen, B1)

Spanish, basic (ZESS Göttingen, A1)

Computer applications

Microsoft Office

Scientific data processing (ChemDraw, Origin, Topspin, MestreNova, PMFIST, Avogadro)

Video and image data processing (Photoshop CS2, Magix Video Deluxe Pro)

Administrative (Admin) work in the group of Prof. Dr. D. Stalke since $04 / 15$

Basic Programming (Java, Delphi, HTML)

Expertise

According to $₫ 5$ Chemikalienverbotsverordnung

\section{Conference participations}

Niedersächsisches Katalyse Symposium (NiKaS), 21.-22.10.2010 in Göttingen (Poster)

Title: „NMR-spectroscopic studies of ammonia borane and its derivatives”

Center of Materials Crystallography (CMC) workshop, 09.-10.10.2014 in Aarhus (Talk)

Title: „Understanding Grignard Aggregation by DOSY NMR”

Jungchemikerforum (JCF), 15.-20.04.2015 in Göttingen (Poster)

European congress on magnetic resonance (Euromar), 05.-09.07.2015 in Prague (Poster; travel grant)

Title: „ECCs for DOSY NMR as valuable tool in understanding aggregation of Grignard compounds and alkali cyclopentadienes"

18. Norddeutsches Doktorandenkolloquium (NDDK), 07.-08.09.2015 in Goslar (Poster)

Center of Materials Crystallography (CMC) meeting, 29.01.2016 in Göttingen (Talk)

Title: „Update on Molecular Weight Determination by ECC-DOSY NMR”

Practical Applications of NMR in Industry Conference (PANIC), 15.-18.02.2016 in Houston, TX, USA (Talk; travel grant)

Title: „ECC-DOSY: A powerful tool to determine molecular weights of small molecule aggregates” 


\section{Scientific Publications in Print}

1. A. Visscher, S. Bachmann, C. Schnegelsberg, T. Teuteberg, R. A. Mata, D. Stalke, „Highly selective and sensitive fluorescence detection of $\mathrm{Zn}^{2+}$ and $C d^{2+}$ ions by using an acridine sensor", Dalton Trans. 2016, 45, 5689-5699.

2. S. Bachmann, R. Neufeld, M. Dzemski, D. Stalke, „New External Calibration Curves (ECCs) for the Estimation of Molecular Weights in Various Common NMR Solvents", Chem. Eur. J. 2016, 22, 84628465.

3. C. Schnegelsberg, S. Bachmann, M. Kolter, T. Auth, M. John, D. Stalke, K. Koszinowski, „Association and Dissociation of Grignard Reagents $\mathrm{RMgCl}$ and Their Turbo Variant RMgCl-LiCl', Chem. Eur. J. 2016, 22, 7752-7762.

4. S. Bachmann, B. Gernert, D. Stalke, „Solution structures of alkali metal cyclopentadienides in THF estimated by ECC-DOSY NMR-spectroscopy (incl. software)", Chem. Commun. 2016, 52, 1286112864 . 